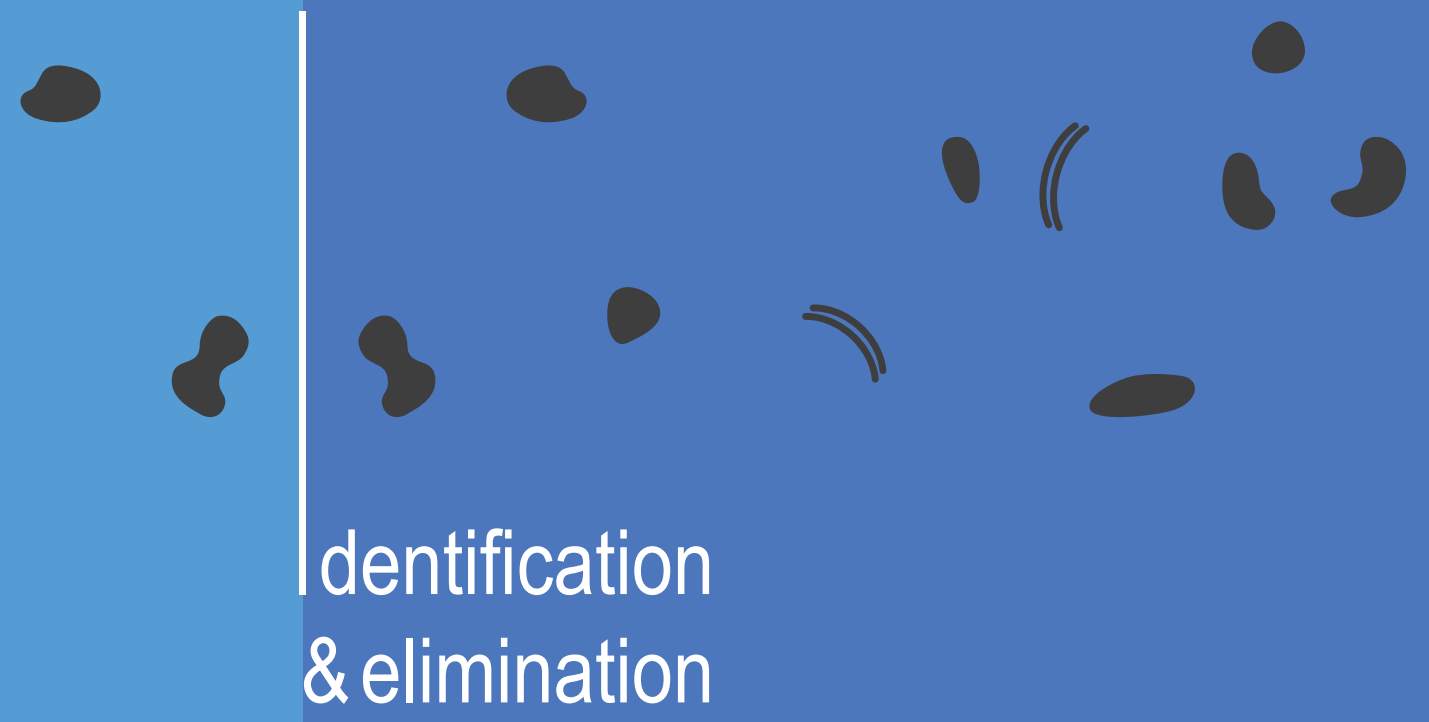

of reflection artifacts

in biomedical photoacoustic imaging

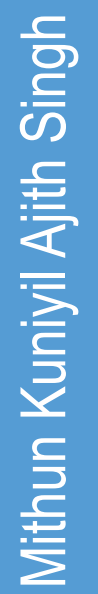




\section{IDENTIFICATION AND ELIMINATION OF}

REFLECTION ARTIFACTS IN

\section{BIOMEDICAL PHOTOACOUSTIC IMAGING}

Mithun Kuniyil Ajith Singh 


\section{Thesis committee members:}

$\begin{array}{lll}\text { Prof. dr. ir. } & \text { W. Steenbergen } & \text { University of Twente (Promotor) } \\ \text { Prof. dr. ir. } & \text { J.W.M. Hilgenkamp } & \text { University of Twente (Chairman and Dean) } \\ \text { Prof. dr. ing. } & \text { G. Schmitz } & \text { Ruhr University Bochum } \\ \text { Prof. dr. ir. } & \text { C.L. de Korte } & \text { Radboud University } \\ \text { Prof. dr. ir. } & \text { C.H. Slump } & \text { University of Twente } \\ \text { Dr. ing. } & \text { P.J. Brands } & \text { ESAOTE Europe BV } \\ \text { Dr. ir. } & \text { A.P. Berkhoff } & \text { University of Twente }\end{array}$

The work described in this thesis is performed at the Biomedical Photonic Imaging (BMPI) Group, MIRA Institute for Biomedical technology and Technical Medicine, Faculty of Science and Technology, University of Twente, P.O. box 217, 7500 AE, Enschede, The Netherlands.

The research leading to this thesis has received funding from the European Community's Seventh Framework Programme (FP7/2007-2013) under grant agreement n 318067.
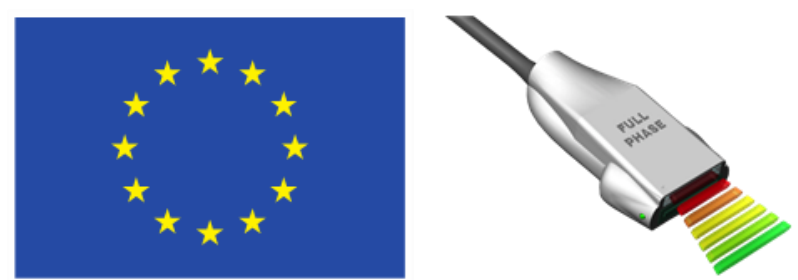

Cover design:

Printed by:

ISBN:

DOI:

This thesis can be downloaded from:
Sjoukje Schoustra

Gildeprint

978-90-365-4262-3

10.3990/1.9789036542623

https://doi.org/10.3990/1.9789036542623

Copyright (C) 2016, by M. Kuniyil Ajith Singh, All rights reserved. No part of this book may be reproduced or transmitted, in any form or by any means, electronically or mechanically, including photocopying, microfilming, and recording, or by any information storage or retrieval system, without prior consent from the author. E-mail: kasmithun@gmail.com 


\title{
IDENTIFICATION AND ELIMINATION OF REFLECTION ARTIFACTS IN BIOMEDICAL PHOTOACOUSTIC IMAGING
}

\section{DISSERTATION}

\author{
to obtain
}

the degree of doctor at the University of Twente, on the authority of the rector magnificus,

Prof. dr. T.T.M. Palstra, on account of the decision of the graduation committee,

to be publicly defended on Thursday, the $8^{\text {th }}$ of December 2016 at 16:45

by

\section{Mithun Kuniyil Ajith Singh}

\author{
born on $14^{\text {th }}$ of June, 1985 \\ in Kozhikode, India
}


This thesis has been approved by:

Prof. dr. ir.

W. Steenbergen

Promotor 
In loving memory of my father, Ajith Singh. K.S (1956-2015) 



\section{Contents}

$1 \quad$ Introduction ........................................................................................................................... 1

1.1 Reflection artifacts in biomedical photoacoustic imaging ........................... 2

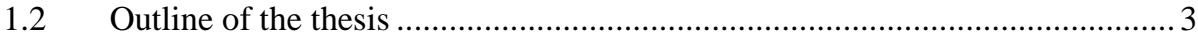

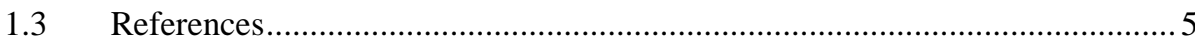

2 Handheld probe-based dual mode ultrasound/photoacoustics for biomedical

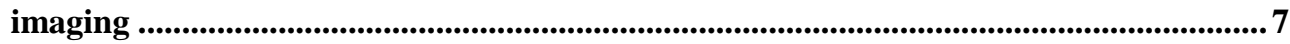

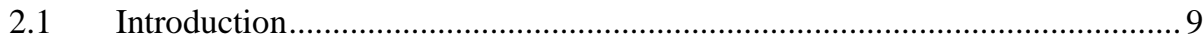

2.2 Handheld probe-based US/PA systems and applications ........................... 17

2.3 Concluding remarks, envisioned improvements and applications .................50

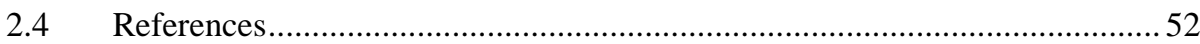

3 Photoacoustic-guided focused ultrasound (PAFUSion) for identifying reflection

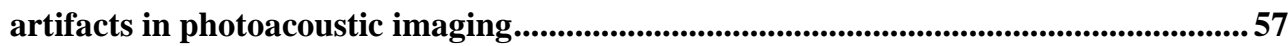

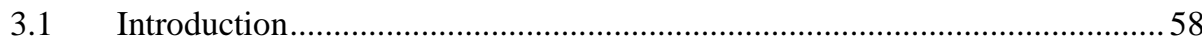

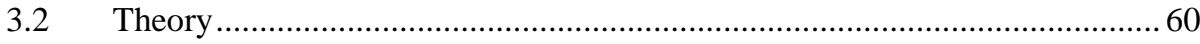

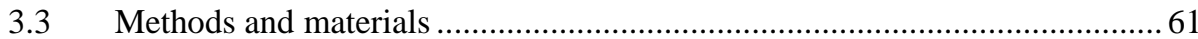

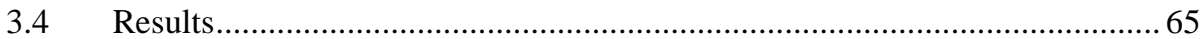

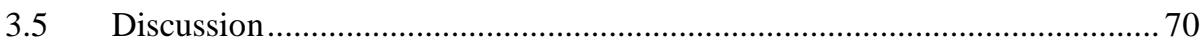

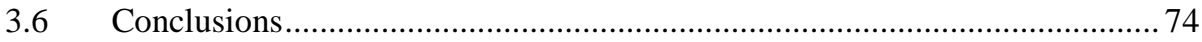

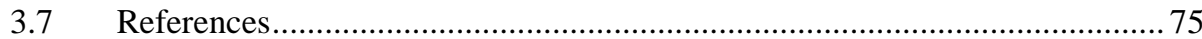

4 In vivo demonstration of reflection artifact reduction in photoacoustic imaging using synthetic aperture photoacoustic-guided focused ultrasound (PAFUSion) ........ 77

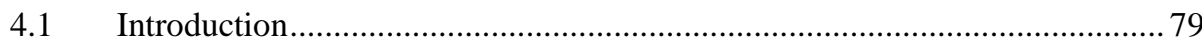

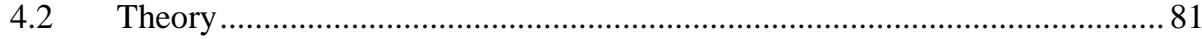

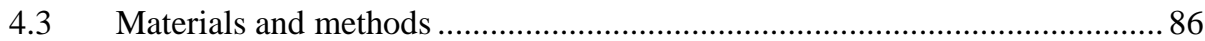

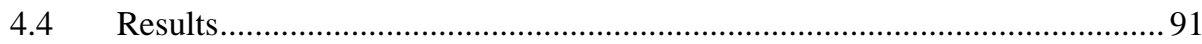

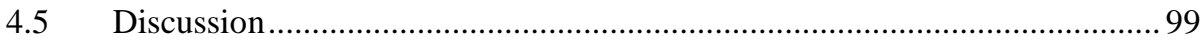

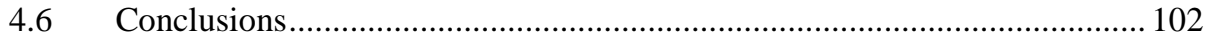

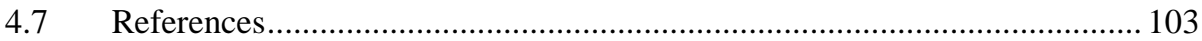


5 Photoacoustic reflection artifact reduction using photoacoustic-guided focused ultrasound: Comparison between plane-wave and element-by-element synthetic backpropagation approach .......................................................................................105

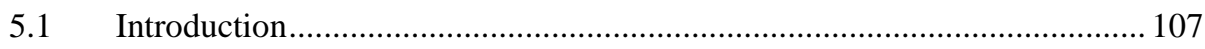

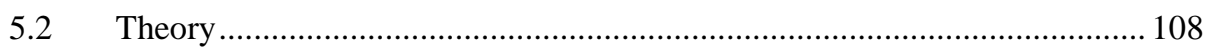

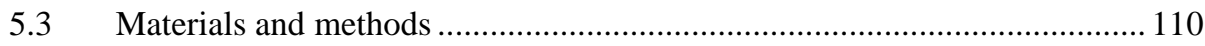

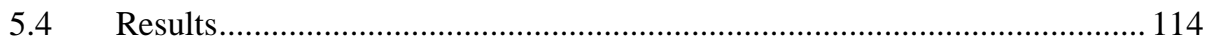

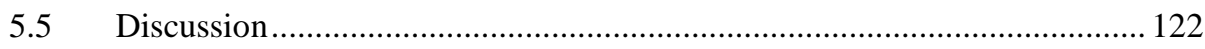

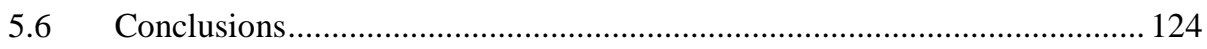

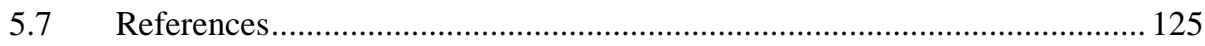

6 Photoacoustic-guided focused ultrasound for accurate visualization of brachytherapy seeds with the photoacoustic needle ......................................................... 127

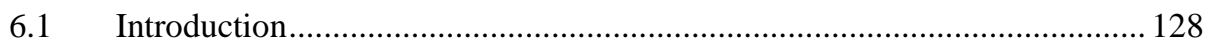

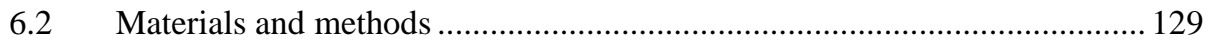

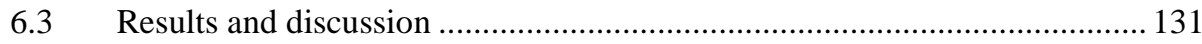

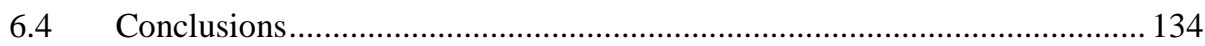

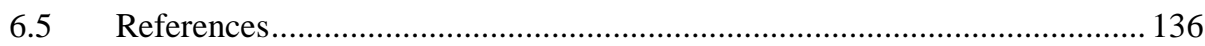

$7 \quad$ Summary and outlook ................................................................................................. 137

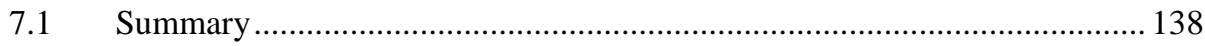

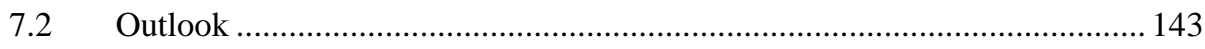

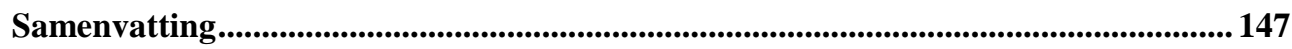

Acknowledgements ................................................................................................................. 153

About the author................................................................................................................. 157

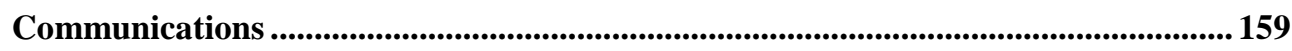

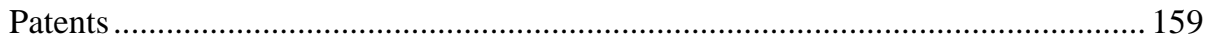

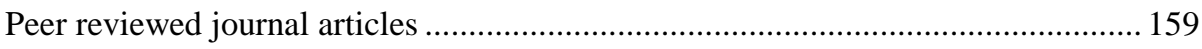

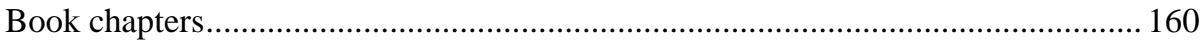

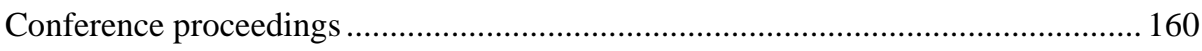

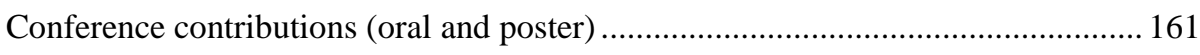




\section{Introduction}

This thesis presents a novel method called PAFUSion (photoacoustic-guided focused ultrasound), for identification and elimination of reflection artifacts, an important problem in biomedical photoacoustic imaging. This chapter briefly discusses the problem of reflection artifacts in photoacoustic imaging, followed by the outline of the thesis. 


\subsection{Reflection artifacts in biomedical photoacoustic imaging}

Photoacoustic (PA) or optoacoustic imaging is a hybrid imaging modality that acoustically detects optical absorption contrast via the PA effect, a physical phenomenon that converts absorbed optical energy into acoustic energy [1]. PA imaging is one of the fastest growing fields in biomedical optics, and has emerged as a powerful biomedical imaging modality in the last decade [2]. This technique offers tremendous potential for wide range of clinical and pre-clinical applications [3].

PA imaging can be easily combined with pulse-echo ultrasound (US) imaging, since the detection part required for both techniques is same. A dual mode system providing complementary contrast offers the advantages of both modalities. A US image superimposed on a PA image offers anatomical and structural information combined with unprecedented functional details, which consequently has the potential to improve specificity and sensitivity in diagnosing various diseases [4]. Bearing in mind the flexibility in imaging bony and acoustically attenuating soft tissue, and ease of clinical acceptance, handheld probes utilizing epi-mode geometry are the best suited for dual mode PA/US imaging. In this case, optical components are either integrated [5] or attached to the US probe and the light illumination is done from the same side where PA signals are detected.

Handheld US/PA imaging with epi-illumination however has an important drawback; high light fluence beneath and proximal to the US probe generates PA transients from superficial optical absorbers, which propagate into the tissue and reflect off echogenic structures to generate reflection artifacts [6-9]. These artifacts can significantly reduce the contrast and imaging depth. In addition, reflection artifacts may appear in the region of interest and be misjudged as an optically absorbing feature, for example: a blood vessel, an inflammation or a cancerous tissue.

Reflection artifacts are an important problem in interstitial-illumination PA imaging also. In interstitial PA imaging, pulsed light is applied in tissue via an optical fiber in a cutting needle with external US detection [10]. In this case, high PA signals from the optical fiber/needle tip act as US transmitters, which traverse into tissue and reflect off echogenic structures to generate reflection artifacts. In any application of minimally invasive PA imaging, this is known to be a critical problem [11]. 
For clinically relevant and acceptable deep-tissue PA imaging, identification and elimination of refection artifacts is of paramount importance. The problem of reflection artifacts (echo clutter) has been identified by the community and some methods have been studied for tackling this [6-9]. LOVIT [7] and DCA [6] aim at reducing reflection artifacts generated below a certain depth (typically below $15 \mathrm{~mm}$ ). Since they require a local tissue displacement relative to the skin surface, they are not suitable for reducing artifacts close to the skin. This is a critical drawback with low-energy integrated systems that are designed for a shallow imaging depth [5], where the in-plane light illumination results in strong reflections of the PA transients generated in the skin melanin layer. Schwab et al [8] utilized a modelbased approach in which US transmissions were used to mimic the reflected PA waves. They derived a mathematical relation in frequency domain that links the plane-wave US measurements and a reflection-artifact-free PA measurement to the actual PA measurement. This relation is inverted in order to attain a reflection-artifact-free PA measurement. However, real-time feasibility and in vivo capability are not demonstrated by them.

\subsection{Outline of the thesis}

The aim of this thesis is to develop a clinically suitable technique for solving the problem of reflection artifacts in biomedical PA imaging. To achieve this goal, this thesis describes a novel method called photoacoustic-guided focused ultrasound (PAFUSion), which is based on the combination of PA and US imaging. In its general form, PAFUSion mimics the inward-travelling PA wavefield from the optical absorbers and the resulting reflection artifacts using US transmissions. At first, as a proof of concept of the method, focused US was used to mimic the inward propagating PA wavefield from point-like optical absorbers, and reflection artifacts caused by them were identified experimentally. Based on promising results from the proof of concept experiments that demonstrated the potential of PAFUSion in identifying reflection artifacts, we improved the method by using different variants of US transmissions (synthetic aperture and plane-wave US measurements) for mimicking the PA wavefields from spatially distributed PA sources. In this thesis, the potential of PAFUSion in identifying and eliminating reflection artifacts in biomedical PA imaging (epi-mode and interstitial illumination PA imaging) is demonstrated using simulations, phantom experiments, ex-vivo studies, and in vivo measurements on human volunteers. 
Chapter 2 is a review which details the fundamentals of PA/US imaging and also depicts the importance of handheld probe-based dual mode PA/US systems. This review pays particular attention to the engineering aspects of systems developed by different groups and the range of clinical applications demonstrated by them.

In chapter 3, the basic idea of PAFUSion is presented with proof of concept simulations and experiments. It is experimentally validated that focused US pulses can be used to mimic the inward propagating PA wavefield from point-like optical absorbers and the reflection artifacts caused by them. All the advantages and shortcomings of the proposed single focus PAFUSion approach are also detailed in this chapter.

Chapter 4 presents an improved version of PAFUSion where PA signals are backpropagated to imitate the inward travelling wavefield and thus the reflection artifacts. The backpropagation is performed in a synthetic way based on the pulse-echo acquisitions after transmission on each single element of the transducer array (synthetic aperture PAFUSion). Phantom experiments and in vivo measurements on the forearm of healthy volunteers are presented to demonstrate the impact and importance of PAFUSion in the field of biomedical photoacoustics.

In chapter 5, an alternative variant of PAFUSion is proposed, in which virtual backpropagation of PA signals is based on multi-angled plane-wave US measurements. In this work, plane-wave and synthetic aperture PAFUSion are implemented in a handheld US/PA imaging system and feasibility of reflection artifact reduction is demonstrated in phantoms and in vivo measurement on a human finger using both approaches. Both the variants of PAFUSion are quantitatively compared in terms of artifact reduction efficiency and acquisition speed.

Chapter 6 proposes the use of PAFUSion in the context of interstitial illumination PA imaging. The potential of PAFUSion in identifying and reducing reflection artifacts with the use of PA-needle in the enhanced visualization of brachytherapy seeds is studied using tissue mimicking phantom and an ex vivo porcine tissue embedded with brachytherapy seeds.

Chapter 7 summarizes the research presented in this thesis and gives recommendations for future work to improve upon the proposed technique. 


\subsection{References}

1. A.G. Bell, On the production and reproduction of sound by light. American journal of Science, 1880. 20: p. 305-324.

2. Y. Zhou, J. Yao, and L.V. Wang, Tutorial on photoacoustic tomography. J Biomed Opt, 2016. 21(6): p. 61007.

3. L.V. Wang and J. Yao, A practical guide to photoacoustic tomography in the life sciences. Nat Methods, 2016. 13(8): p. 627-38.

4. R. Bouchard, O. Sahin, and S. Emelianov, Ultrasound-guided photoacoustic imaging: current state and future development. IEEE Trans Ultrason Ferroelectr Freq Control, 2014. 61(3): p. 450-66.

5. K. Daoudi, et al., Handheld probe integrating laser diode and ultrasound transducer array for ultrasound/photoacoustic dual modality imaging. Optics Express, 2014. 22(21): p. 26365-26374.

6. M. Jaeger, et al., Deformation-compensated averaging for clutter reduction in epiphotoacoustic imaging in vivo. Journal of Biomedical Optics, 2012. 17(6).

7. M. Jaeger, J.C. Bamber, and M. Frenz, Clutter elimination for deep clinical optoacoustic imaging using localised vibration tagging (LOVIT). Photoacoustics, 2013. 1(2): p. 19-29.

8. H.M. Schwab, M.F. Beckmann, and G. Schmitz, Photoacoustic clutter reduction by inversion of a linear scatter model using plane wave ultrasound measurements. Biomedical Optics Express, 2016. 7(4): p. 1468-1478.

9. E.J. Alles, M. Jaeger, and J.C. Bamber, Photoacoustic Clutter Reduction Using Short-Lag Spatial Coherence Weighted Imaging. 2014 Ieee International Ultrasonics Symposium (Ius), 2014: p. 41-44.

10. D. Piras, et al., Photoacoustic needle: minimally invasive guidance to biopsy. J Biomed Opt, 2013. 18(7): p. 070502.

11. M.A.L. Bell, et al., In vivo visualization of prostate brachytherapy seeds with photoacoustic imaging. Journal of Biomedical Optics, 2014. 19(12). 


\section{2}

\section{Handheld probe-based dual}

\section{mode ultrasound/photoacoustics}

\section{for biomedical imaging ${ }^{1}$}

Photoacoustic imaging is a promising biomedical imaging modality that has emerged over the last decade. In this method, imaging is performed using pulsed far-red or near-infrared light. This light while scattering through soft tissue is absorbed at specific locations by certain molecules such as hemoglobin in blood. The absorbed energy is converted into heat; the subsequent thermoelastic expansion causes ultrasound to be produced from the absorbing region. The ultrasound is measured at the surface of tissue using ultrasound detectors and the acquired signals are used to reconstruct the location and spatial details of the absorber. Photoacoustic imaging thus combines the advantages of optical and ultrasound imaging, providing excellent optical spectroscopic contrast with ultrasonic resolution. While ultrasound imaging utilizes acoustic impedance mismatches in tissue for its signals to provide structural details, photoacoustic imaging extracts functional information based on optical absorption by chromophores, predominantly blood, and often exogenous contrast agents. Since photoacoustic imaging involves ultrasound detection, it can be seamlessly implemented in a commercially available ultrasound scanner to perform dual mode ultrasound/photoacoustic imaging, which is a promising translational medical diagnostic technique. These dual mode systems providing complementary contrast hold potential for myriad of clinical applications. Handheld dual mode ultrasound/photoacoustic probes use reflection mode imaging geometry, where light irradiation is done from the same side where

\footnotetext{
1 This chapter has been published as: M. Kuniyil Ajith Singh, W. Steenbergen, and S. Manohar, "Handheld Probe-Based Dual Mode Ultrasound/Photoacoustics for Biomedical Imaging," in Frontiers in Biophotonics for Translational Medicine M. Olivo, and U. S. Dinish, Eds., pp. 209-247, Springer Singapore (2016).
} 
photoacoustic signals are detected. These epi-style handheld probe-based imaging setup delivers flexibility in imaging different body parts using the same probe. This review details the fundamentals of ultrasound/photoacoustic imaging and also depicts the importance of handheld probe-based dual mode ultrasound/photoacoustic imaging systems. Particular attention is paid to the engineering aspects of systems developed by different groups and range of clinical applications demonstrated by them. 


\subsection{Introduction}

Medical imaging provides crucial information that assists decision making for choosing the course of action and suitable therapy. Imaging can contribute to detection of the occult disease, and accurate diagnosis, which can enable timely intervention, precluding complex medication and surgery at late stages of disease. Imaging technologies strive to achieve this at an affordable cost with minimal patient burden. Examples of modalities that are present in most specialist hospitals are: ultrasound (US) imaging, $\mathrm{x}$-ray imaging, Magnetic Resonance Imaging (MRI), x-ray Computed Tomography, Positron Emission tomography (PET) etc. The techniques can be fundamentally different in the physics of the interaction of the energy probe with tissue, in contrast mechanisms, and in the applications. The technologies are different in terms of initial expense, and infrastructure and maintenance requirements, and in their performance parameters.

Of the methods used, US imaging is largely ubiquitous in clinics owing to its compactness, relative affordability and real-time performance especially for point of care applications. A recent innovation is development of a hybrid modality bringing together US imaging with photoacoustic (optoacoustic) imaging. The latter is a new method that uses pulsed laser light to excite US from within tissue from absorbing structures such as blood vessels. While US imaging has a long history, photoacoustic (PA) imaging is at the stage of early proof-of-principle clinical studies in research hospitals, but is expected to have an impact in health care in the near future. Handheld probe-based dual mode systems combining US and PA imaging holds promise as a potential technique for translational biomedical imaging.

In this chapter, we review various handheld probe-based systems capable of performing both US and PA imaging, and their myriad applications. The first section provides an overview of the existing optical and US imaging techniques, followed by a detailed description of PA imaging. Furthermore, this section briefly discusses the importance of hand held and portable systems in clinical settings. The second section presents the engineering aspects of handheld dual mode US/PA systems currently being investigated and tested, and different applications that are explored and foreseen. 


\subsubsection{Optical imaging}

Optical tissue imaging, makes use of spectroscopic features of light-tissue interactions such as scattering, absorption and polarization [1]. Since many physiological molecules in tissue possess characteristic wavelength-dependent absorptions (Figure 2.1), this interaction is most powerful for interrogating tissues at functional and molecular levels [2]. The molecules of interest are typically hemoglobin $(\mathrm{Hb})$, oxyhemoglobin $\left(\mathrm{HbO}_{2}\right)$, melanin, lipids and water, each with a signature optical absorption spectrum [3].

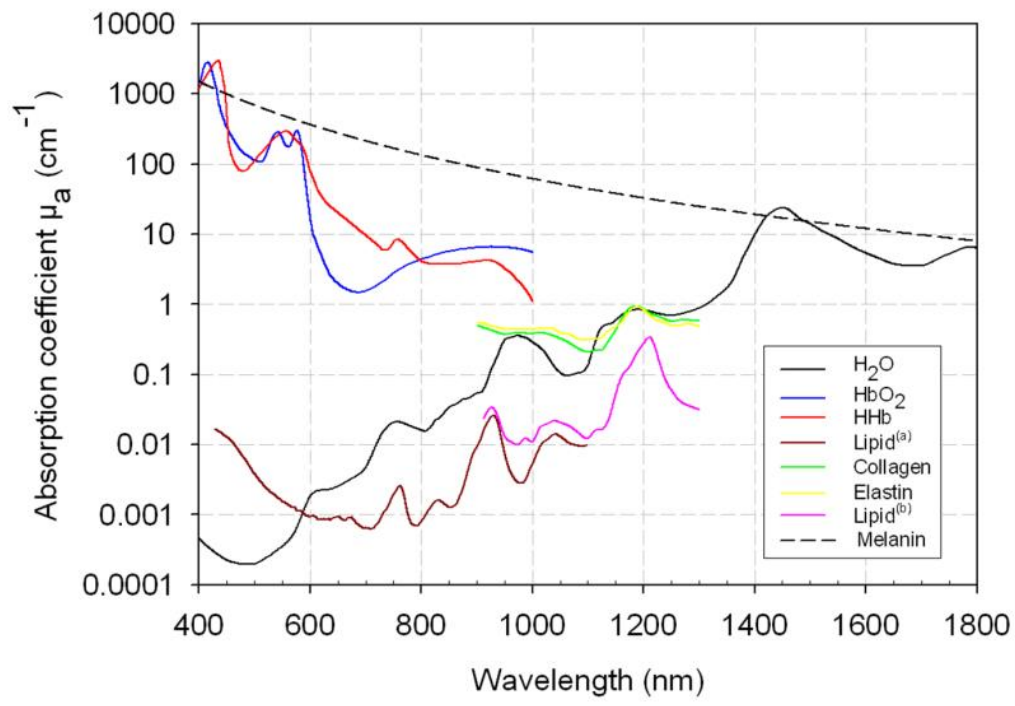

Figure 2.1 Absorption coefficient spectra of endogenous tissue chromophores. Oxyhemoglobin (red line), deoxyhemoglobin (blue line), water ( $80 \%$ by volume in tissue, black line), lipid (a) (20\% by volume in tissue, brown line), lipid (b) (pink line), melanin ( $\mu_{\mathrm{a}}$ corresponds to that in skin, black dashed line), Collagen (green line) and elastin (yellow line) (Reprinted from ref. [4] Beard, P. 2011. Interface Focus 1(4): 60231. With permission from the author and Royal Society Publishing.)

The relative presence of these chromophores can often be linked to different disease conditions. For example, increased hemoglobin can be a sign of angiogenesis, growth of new blood vessels, which is often associated with a variety of oncological [5] and inflammatory diseases [6]. Further, angiogenesis is also a natural tissue response for promoting wound and burn healing [1], and can be a marker for monitoring this process. The ratio of $\mathrm{HbO}$ to total 
hemoglobin $\left(\mathrm{H}_{\mathrm{t}}=\mathrm{Hb}+\mathrm{HbO}\right)$ or oxygen saturation, in the microcirculation, is expected to be lower in cancer due to increased oxygen consumption associated with high metabolism, which could be a marker of the disease [7]. Melanin serves as the marker for melanoma detection and evaluation using optical imaging techniques.

The rich spectroscopic contrast of these molecules and their relation to a spectrum of diseases make optical imaging versatile. However, purely light-based imaging techniques suffer from poor resolution at depths larger than a few $\mathrm{mm}$, due to high scattering in tissue $[8]$.

\subsubsection{Ultrasound imaging}

US imaging is able to visualize anatomy from echoes of US pulses insonifying the tissue [9, 10]. The method has a long history of development with the first US detector arrays in use since the 1970s [11]. One of the latest developments in the field is acquisition of ultrafast frame rates for real-time visualization of flow and transient elastography. This is possible with the use of plane waves in which a large area is insonified with single emission from the entire aperture, with the backscattered echoes detected by all elements for building up the image [12]. Multiple images are acquired by steering the plane waves into the object at various angles, and these are compounded either incoherently to reduce speckle and improve contrast, or coherently to improve resolution. Such an approach can realize several hundreds of frames per sec (fps) with the image quality approaching standard multi-focus US imaging with lower frame rates.

Despite all these developments in technology, US imaging fundamentally suffers from poor soft-tissue contrast. While it has various applications for which it is suited, such as differentiating between solid masses and fluid filled cysts, the method is not sufficiently specific or sensitive [13] for discrimination between malignancies and benign abnormalities. Speckle noise in US images degrades contrast which limits its applicability in imaging heterogeneous environments [14]. However the defining advantages of US imaging are the capability to perform real-time imaging, and the fact that low scattering in tissue allows the phase to be preserved, providing the capability to focus US and generally attain excellent resolution even at larger depths [15]. This is in contrast to optical imaging methods where the scattering is 2-3 orders stronger [16]. Figure 2.2 shows speed and contrast differences of 
conventional focused US and plane wave compounding making use of transmission at 40 angles.
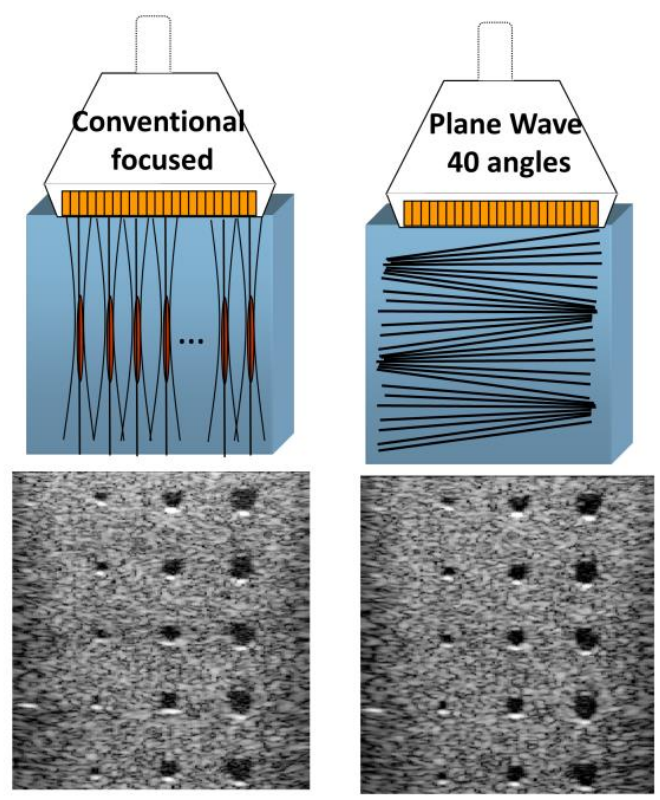

Figure 2.2 Conventional focused and ultrafast US imaging sequence for a typical medical imaging setup (4-cm deep region of interest): (left) conventional focused imaging (128 focused beams and 4 focal depths leading to $25 \mathrm{fps}$ ), (right) plane-wave compounding with 40 angles ( $350 \mathrm{fps}$ ). (Adapted from ref. [12] Tanter, M. and M. Fink, 2014. IEEE Transactions on Ultrasonics, Ferroelectrics and Frequency Control, 61(1): p. 102-19. With permission from the authors and IEEE.)

\subsubsection{Photoacoustic imaging}

In PA imaging, the advantages of the high contrast of optical imaging, and the high resolution of US imaging are brought together. Here pulsed light absorbed by the target emits US by a thermoelastic mechanism. This US is detected and used to build up an image [17]. The effect has its roots in early work by Alexander Graham Bell in 1880 who observed sound generation from certain dark materials exposed to modulated sunlight [18]. The first work on using the pulsed PA effect potentially for medical imaging application was reported in the mid-1990's 
[19-21]. Since then there has been steady growth in the field with several embodiments of the technology, and various demonstrations of potential applications.

\subsubsection{Photoacoustic signal generation process}

Figure 2.3 illustrates the PA effect in biological tissue. Short pulsed laser light is shone on the tissue, and depending on the wavelength the photons will undergo scattering and absorption [4]. This absorbed laser energy initiates a temperature rise, which then leads to an expansion of the absorbing structure. Under stress and thermal confinement conditions, this causes an initial pressure increase and the subsequent emission of US waves, which propagate to the surface where they are detected. Stress confinement is met when the generated PA waves are not travelling longer than the size of light absorber, during the course of the light excitation pulse. This is fulfilled if the sound velocity multiplied by the pulse duration of the laser is small compared to the size of the light absorbing structure. Thermal confinement is met when the heat generated by the absorber is not diffusing more than the size of the absorber during the course of the light excitation pulse. As heat diffusion is usually slower than the propagation of sound, the thermal confinement is fulfilled if stress confinement is fulfilled. Theoretically, only 1 milliKelvin temperature can generate about $800 \mathrm{~Pa}$ pressure rise which then can be detected by a typical ultrasonic transducer [8].

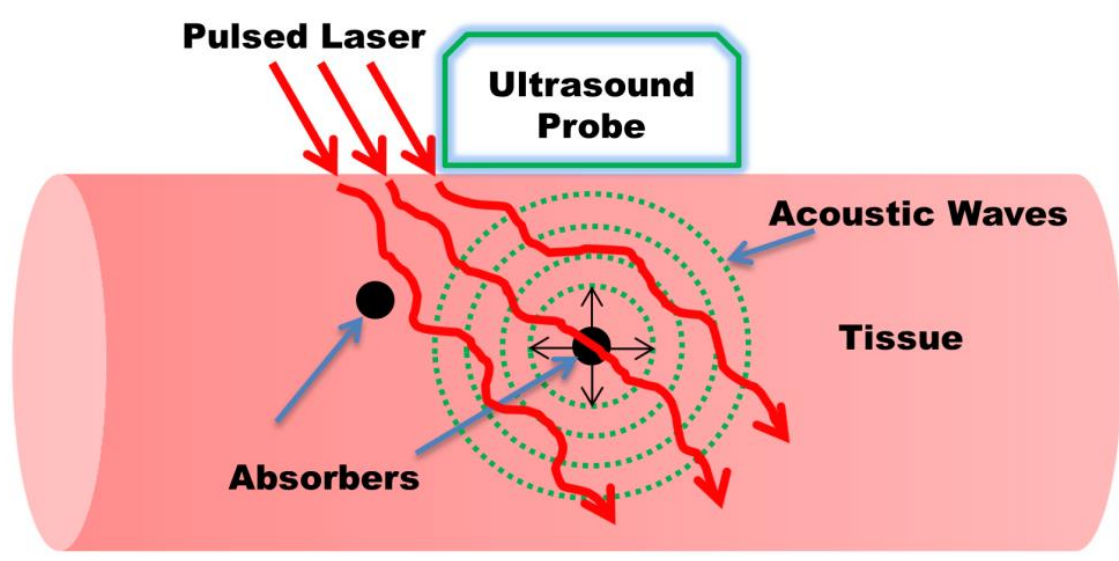

Figure 2.3 Schematic illustration of the PA effect in biological tissue. 
PA signal generation can be regarded as an optical absorption-induced pressure distribution $p_{0}$ from which US waves propagate to the surface of tissue to be transduced into time resolved electric signals using US detectors. Images are formed by acquiring signals from multiple spatial points. Since US undergoes low scattering and absorption, a PA image can be considered in the first approximation as an image space representation of $p_{0}$ [4]. Under conditions of stress and heat confinement, the initial pressure distribution $p_{0}$ at a point $r$ is proportional to $H(r)$, the absorbed optical energy density [22].

$$
p_{0}(r)=\Gamma H(r)
$$

Here $\Gamma$ is the Grüneisen coefficient, a dimensionless thermodynamic constant which describes the conversion efficiency of heat energy to pressure. It is given by $\Gamma=\beta c^{2} / C_{p}$, where $\beta$ is the volume thermal expansion coefficient, $c$ the speed of sound and $C_{p}$ the specific heat capacity at constant pressure. The absorbed optical energy $H(r)$ is the product of the local absorption coefficient $\mu_{a}(r)$ and the optical fluence $\Phi\left(r, \mu_{a}, \mu_{s}, g\right)$ where $\mu_{a}$ and $\mu_{s}$ are the absorption and scattering coefficients over the illuminated tissue volume with $g$ the anisotropy factor. So $p_{0}$ can be written as follows

$$
p_{0}(r)=\Gamma \mu_{a}(r) \Phi\left(r, \mu_{a}, \mu_{s}, g\right)
$$

Equation (2.2) shows that $p_{0}(r)$ while depending explicitly on local $\mu_{a}$ also depends via the fluence globally on $\mu_{a}$ and $\mu_{s}$ in tissue through which photons have traversed to $r$. Thus image contrast is not directly proportional to $\mu_{a}$, but to the product of $\mu_{a}$ and $\Phi$ with the fluence itself depending on $\mu_{a}$ making $p_{0}$ a nonlinear function of $\mu_{a}$ [4]. Further, $p_{0}(r)$ also depends on mechanical and thermodynamic parameters via $\Gamma$ which may also change depending on position [23]. Nevertheless for a qualitative treatment, the PA image can be thought to represent the spatial distribution of absorbed optical energy $H(r)$ in tissue.

\subsubsection{Photoacoustic image reconstruction}

Since the method relies on optical contrast and US propagation-detection, reconstruction tools and analyses borrow from the two areas of diffuse optics and US. Basic PA reconstruction serves to retrieve the spatial distribution of the acoustic sources or locations of optical energy deposition. This is termed the acoustic inversion problem and uses various 
algorithms from the US and sonar world. From the distribution of acoustic sources one would like to estimate a representation of the optical absorption coefficient distribution, but as discussed earlier, this is a non-linear problem. Reconstruction of optical absorption coefficient is termed quantitative PA imaging.

In this review of combined PA-US systems, we focus on basic PA image reconstruction schemes which resemble the methods for reconstructing medical US data. The main differences between US and PA image reconstructions are: 1) the time of flight is half in PA imaging, since the acoustic waves are generated within tissue and not by backscattering of ultrasound as in US imaging, 2) the frequency content of detected signals in PA is different based on the sizes of the absorbers, while that in US imaging is generally the same as the injected frequencies.

A review of the mathematics behind reconstruction approaches in PA imaging is given in Kuchment et al [24]. The reconstruction algorithms are selected based on the geometry of receiving elements, computing speed available etc. As this chapter focuses on handheld probe-based systems, which mainly make use of linear array transducers, discussion about reconstruction algorithms will be limited to those which are practically being applied for these geometries.

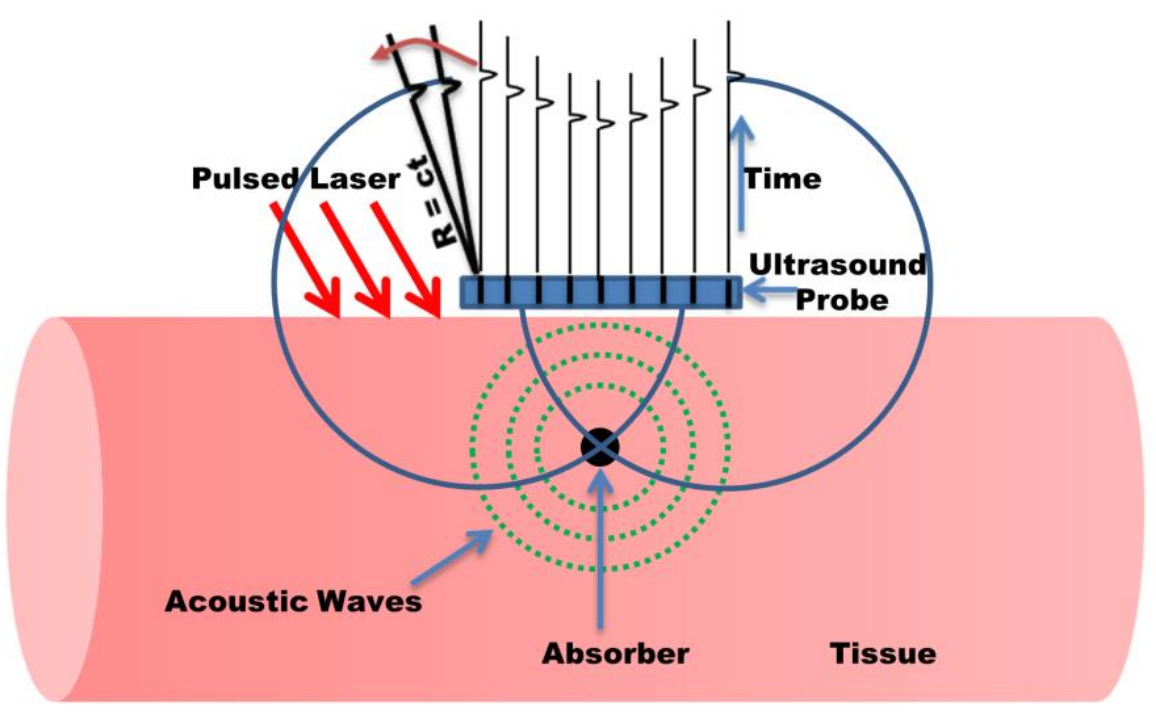

Figure 2.4 Backprojection PAT image reconstruction for a planar detection geometry. 
The filtered back-projection algorithm is commonly applied for PA data due to its simplicity and speed [4]. Figure 2.4 shows the basic idea behind the algorithm for a PA linear/planar detection geometry. In back-projection PA tomography image reconstruction for a planar detection geometry, PA waveforms are recorded by each detector element of the US probe at specific location, spatially resolved using the sound speed $c$ and back-projected over spherical surface of radius $R=c t$ into the imaging volume [4]. The recorded pressure waveforms are time integrated computationally in this case.

The method is analogous to delay and sum beam-forming employed in phased array US imaging [23]. A delay and sum beam-forming algorithm used for image reconstruction in a US imaging system can be used for PA image reconstruction just by reducing the time of flight to half. This easy switching between reconstruction algorithms is important for dual modality PA-US imaging systems. Although both methods give simple and clear description about PA image formation, it is not the finest in terms of precision and computational overhead. The time reversal method is also gaining in popularity due to the availability of a freely available third party MATLAB toolbox, which performs image reconstruction using k-space methods [25].

An algorithm based on harmonic decomposition of the pressure waves arising from the object has been used in linear/planar detection geometries. Since the computation overhead is important, this approach is attractive since most of the processing is done via the fast Fourier transform and a $k$-space interpolation [4]. The use of the frequency-domain approach permits reconstruction and presentation of the resultant image in real-time, a key criterion for a PA system developed around a US scanner. Several groups have reported on fast algorithms based on frequency domain processing [26, 27]. Systems have been presented that can do real time reconstruction of PA images [28-30], and some others based on recent developments in GPU technology which can do real-time PA and US imaging [14].

\subsubsection{Handheld Ultrasound/Photoacoustic Imaging Systems - Motivation}

Imaging tests on patients are usually done using various modalities in sequence to extract the necessary information from the suspect region or organ to decide the course of action. Multimodality imaging combining two complementary techniques, is of interest to reduce the need for multiple or layered imaging. PA imaging can be combined with US imaging with relative ease since the acoustic hardware required for both techniques can be shared. A 
dual mode system combines the advantages of both modalities. A US image superimposed on a PA image can give anatomical and structural information overlaid with rich detail of functions. The versatility of such a system is further improved by the availability of US Doppler imaging and elasticity imaging [31].

In addition to the convenient visualization of complementary information, estimated parameters from US imaging such as speed of sound (SOS) and acoustic attenuation (AA) can be utilized into PA imaging in an iterative framework to improve the accuracy of the reconstruction. This can play an important role in enabling quantitative PA imaging [32, 33].

Further, PA is a new technology seeking to make inroads into the clinic, while US is an accepted modality in healthcare. From the point of view of PA, the incorporation of PA into a dual modality system with US can potentially have relatively easier clinical acceptance [28, 33]. The impact and importance of a system that can perform co-registered US and PA imaging using a handheld probe, which is clinically acceptable, is high considering the vital point of care applications in diagnostic imaging and treatment monitoring.

\subsection{Handheld probe-based US/PA systems and applications}

\subsubsection{The University of Bern system}

The first report of a combined PA-US system using a handheld probe was from the Bern group [27] in collaboration with the Fraunhofer Institute (St. Ingbert, Germany). A custom made high-speed US system was developed that could simultaneously perform PA imaging. The 128-element linear array used had a center frequency of 7.5 MHz (LA/7.5/128/ULS, Vermon S.A, France). Light illumination was provided from a Q-switched Alexandrite laser emitting short near-infrared laser pulses (60 ns) at a wavelength of $760 \mathrm{~nm}$.

The laser light was coupled to a $400 \mu \mathrm{m}$ optical fiber, and using a prism and two mirrors (Figure 2.5) was split into two beams and delivered to the object from either side of the probe, giving a homogeneously illuminated spot of diameter $25 \mathrm{~mm}$. The radiant exposure was under $5 \mathrm{~mJ} / \mathrm{cm}^{2}$, below the maximum permissible exposure (MPE) of $20 \mathrm{~mJ} / \mathrm{cm}^{2}$. 


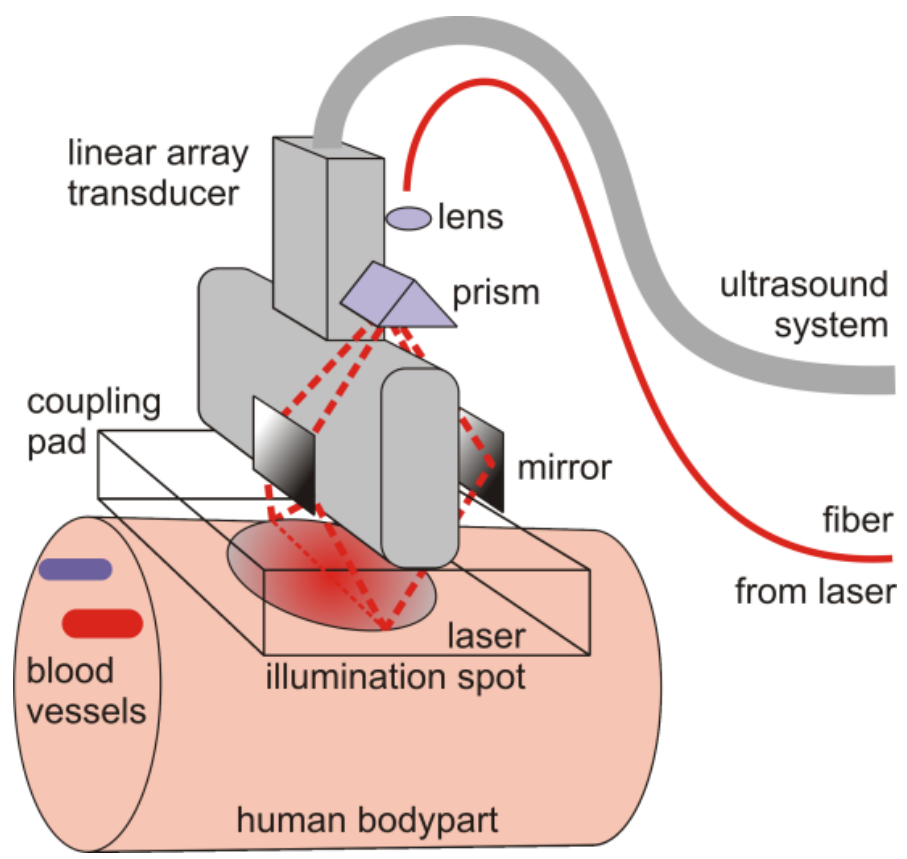

Figure 2.5 Dual mode PA and US real-time imaging setup. (Reprinted from ref. [27] Niederhauser, J.J. et al. 2005. IEEE Transactions on Medical Imaging, 24(4): p. 436440. With permission from the authors and IEEE.)

The system was connected to a dual processor computer, which allowed for real time reconstruction at 7.5 fps using a modified Fourier reconstruction algorithm [34]. For USechography, a conventional digital beam-former with time gain compensation (TGC) amplifier and dynamic aperture were used on 16 channels. Proper adjustments were done in the reconstruction software between the two modalities. Since parallel acquisition was used, only one pulse was enough for recording a complete frame, and reconstruction was done in less than $100 \mathrm{~ms}$. The system had the capability of displaying a PA image alongside the classical US image. With this system, the lateral resolution achievable was defined by the NA of the 'lens' formed by all active detector elements and the average US wavelength (associated with the centre frequency of the array), and the axial resolution of $0.3 \mathrm{~mm}$ was defined by the bandwidth of the transducer $(5.5 \mathrm{MHz})$. The transducer was sensitive to blood vessels in the range of $100 \mu \mathrm{m}$ to $300 \mu \mathrm{m}$ corresponding to signal frequencies of $4.5 \mathrm{MHz}$ to $10 \mathrm{MHz}$ within the bandwidth. The system was not sensitive to smaller vessels compared to 
the larger ones, and the larger vessels also showed distortions such as edge oscillations in the reconstructions.

The system demonstrated the importance of parallel data acquisition and fast image reconstruction. However the system made use of a custom-made data acquisition set up which would more likely be expensive when compared to systems derived from preexisting commercial US scanners.

Figure 2.6a shows the PA image and Figure 2.6b the US image of a healthy human index finger measured using this system. The images cover a $2.6 \times 2.0 \mathrm{~cm}$ area (ticks every $\mathrm{cm}$ ) with the transducer at the top of the images. On the PA image (left) the skin is visible as an arc shaped black line (s). At $1 \mathrm{~mm}$ below the skin the $V v$. digitales palmares are visible as two black dots on either side of the finger (arrows). They are accompanied by the corresponding Aa. digitales palmares propriae, which appear much fainter (tiny dots besides veins) because of the reduced absorption of oxyhemoglobin at $760 \mathrm{~nm}$. Below the skin, at $3 \mathrm{~mm}$ depth, a blood perfused region possibly the periosteum is visible. The four vessels and the periosteum are not visible in the corresponding US image (right). The US image is predominated by the echo signal from the skin (s) and the large echo signal from the bone $5 \mathrm{~mm}$ below it. The skin surface position matches well on both images; it is, however, obvious that the two methods extract complementary information.
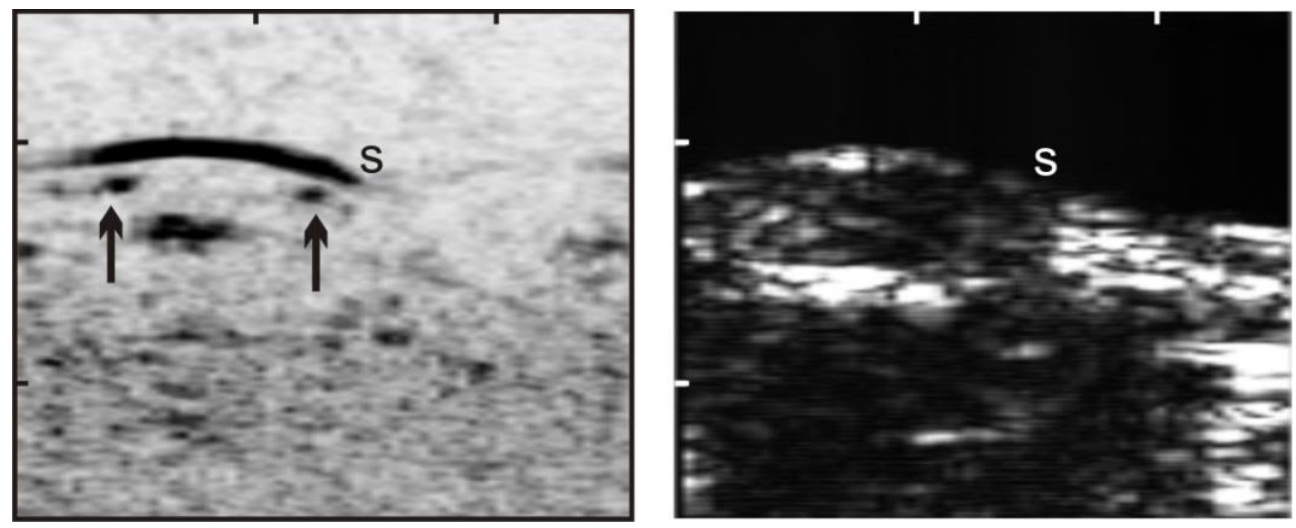

Figure 2.6 (a) PA image and (b) US image of a human index finger (palm toward transducer). (Reprinted from ref. [27] Niederhauser, J.J. et al. 2005. IEEE Transactions on Medical Imaging, 24(4): p. 436-440. With permission from the authors and IEEE.) 


\subsubsection{The Twente systems}

The Twente group in collaboration with ESAOTE Europe BV developed a dual modality imaging system, one of the first attempts in using a commercial US scanner (Picus, ESAOTE Europe BV, Maastricht, the Netherlands) for PA imaging [30, 35]. The system used a linear array (L10-5, $40 \mathrm{~mm}, 7.5 \mathrm{MHz}$ central frequency, 128 elements, ESAOTE Europe BV) transducer. Light pulses (8 ns) from a $1 \mathrm{kHz} \mathrm{Nd:YAG} \mathrm{laser} \mathrm{(1064} \mathrm{nm)} \mathrm{were} \mathrm{coupled} \mathrm{to} \mathrm{a}$ $600-\mu \mathrm{m}$ optical fiber which terminated in an optical module fitted to the US array (Figure 2.7B). The custom -made module was composed of a beam expander, a cylindrical lens and a stationary mirror that illuminated an area of $5 \times 20 \mathrm{~mm}^{2}$ (Figure 2.7C).
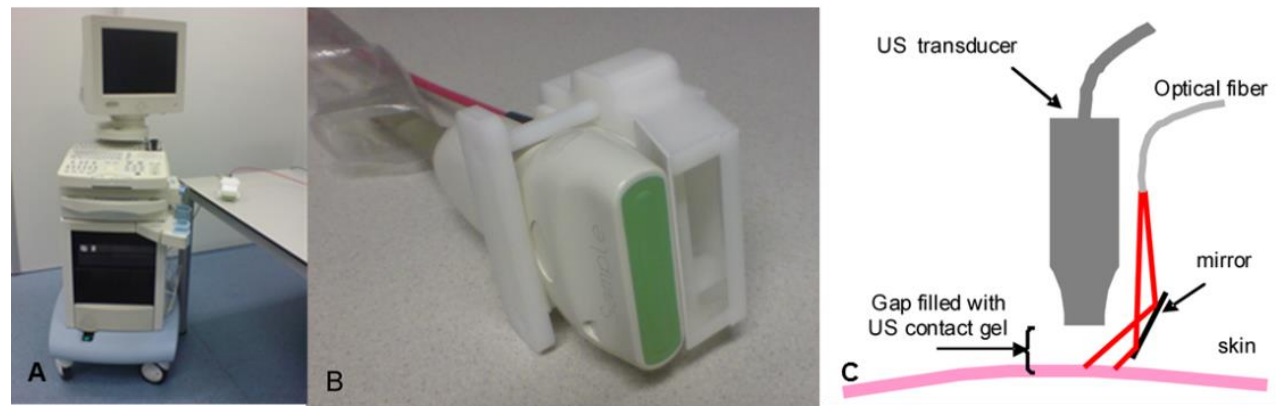

Figure 2.7 (A) US system (Picus, ESAOTE Europe BV, Maastricht the Netherlands),

(B) Optical module connected to US probe, (C) Schematic overview of optical system connected to US probe. (Adapted from ref. [30, 35] Kolkman, R.G.M. et al. 2008.

Journal of Biomedical Optics, 13(5):050510 and Kolkman, R.G.M. et al. 2009.

Proceedings. SPIE 7177,717704. With permission from the authors and SPIE.)

The changes made to incorporate PA imaging, were synchronization of the dataacquisition with the firing of the laser and an option to switch-off the emission of US. A sequential line-by-line scanning approach was employed, requiring 128 laser pulses for a frame. At a pulse repetition rate of $1 \mathrm{kHz}$, this resulted in a frame rate of $8 \mathrm{fps}$. All the required modifications mentioned and changes in reconstruction algorithm were achieved without affecting the system's performance for conventional US imaging.

Center frequency $(7.5 \mathrm{MHz})$ and bandwidth $(75 \%-6 \mathrm{~dB})$ of the US transducer limited the sensitivity of the system to visualize structures larger than $100 \mu \mathrm{m}$. This real-time US/PA system had the advantage that it made use of a commercial US scanner, which precluded the 
need for high-speed digitizers and computers for data acquisition and reconstruction of images.

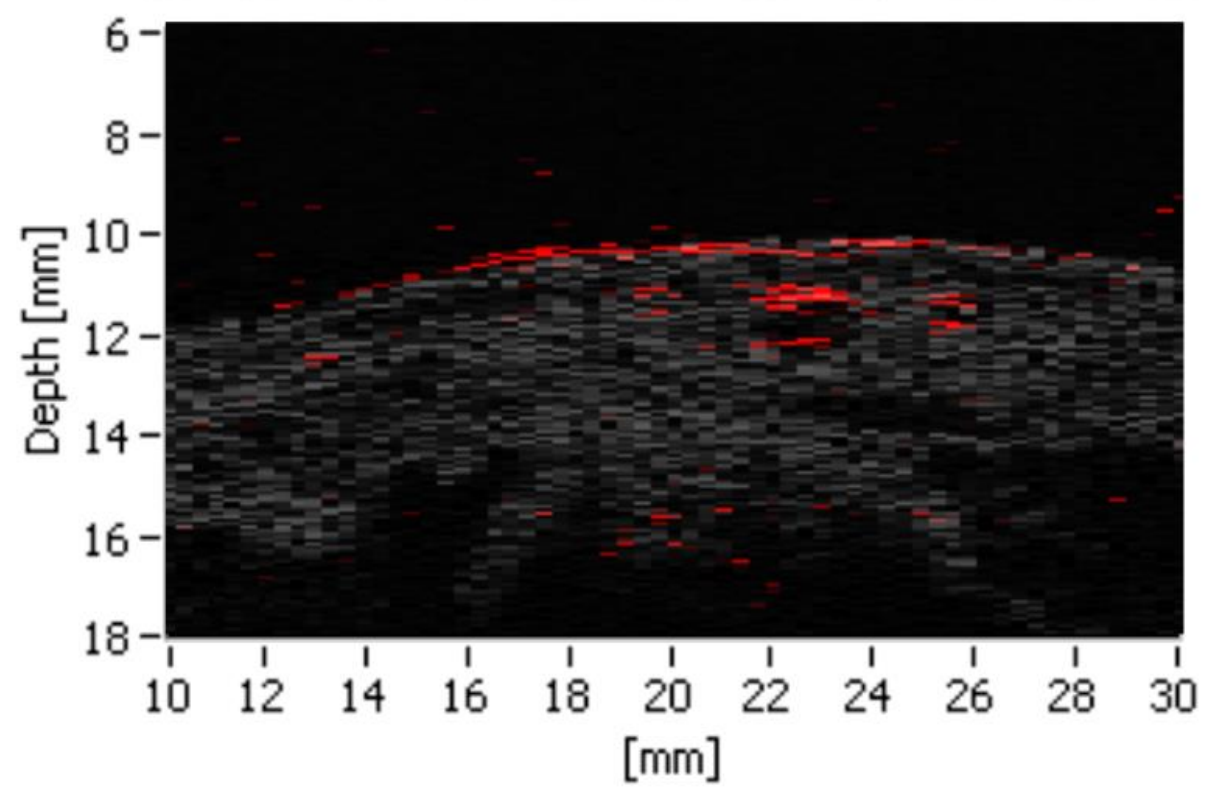

Figure 2.8 Combined US (gray scale) and PA (red) image showing absorption in blood vessels and the skin. (Adapted from ref. [35] Kolkman, R.G.M. et al. 2008. Journal of Biomedical Optics, 13(5):050510. With permission from the authors and SPIE.)

Overlay images of measurements on the dorsal side of a volunteer's hand are shown in Figure 2.8. PA signals from the skin and blood vessels are clearly visible and US here supports to identify boundaries and bone. The largest blood vessel (axial diameter $1.22 \mathrm{~mm}$ ) visualized in the PA data $(x=22 \mathrm{~mm}$, depth $=12 \mathrm{~mm})$ is visible in the US image as a hypoechogenic region. Smaller vessels present in the PA image are not visible in US. The bone is visualized as a semicircular structure with low backscatter intensity in the US image $(\mathrm{x}=16$ to $26 \mathrm{~mm}$, depth $=15$ to $18 \mathrm{~mm})$. PA signal reflection artifacts are present in the image at a depth of around 16-18 mm. US imaging plays a crucial role in identifying and potentially correcting these PA reflection artifacts by identifying the acoustic reflectors responsible for them.

The Twente group recently reported on a fully integrated hand-held probe, combining an US array transducer and a diode laser module in a compact and ergonomic design that can 
perform dual modality US/PA imaging [36]. The probe consists of 128 elements, each with a length of $5 \mathrm{~mm}$ and a pitch of $0.245 \mathrm{~mm}$. The array has a central frequency of $7.5 \mathrm{MHz}$ and a measured $-6 \mathrm{~dB}$ bandwidth of around $100 \%$. This dual modality probe makes use of a commercially available portable US scanner (MyLabOne, ESAOTE Europe B.V, Maastricht, the Netherlands) modified for incorporating a diode laser with a laser fluence of up to 1.3 $\mathrm{mJ} / \mathrm{cm}^{2}$ on the skin with an illumination spot size of $18.2 \times 2.3 \mathrm{~mm}^{2}$. For PA imaging, the diode laser module was triggered using stable hardware signal from the system. This trigger signal and US transmission was controlled using system software for toggling between US and PA imaging. This system achieved a frame rate of $10 \mathrm{fps}$ after averaging 20 frames during PA imaging. Line by line US measurement of the same area resulted in a screen refresh rate of $80 \mathrm{fps}$. The frame rate of this system was limited by the maximum permissible exposure (MPE) of laser light on tissue. Figure 2.9 shows the photograph of the portable dual modality US/PA system. This novel system is affordable, portable and thus undeniably a step towards clinically acceptable US/PA system.

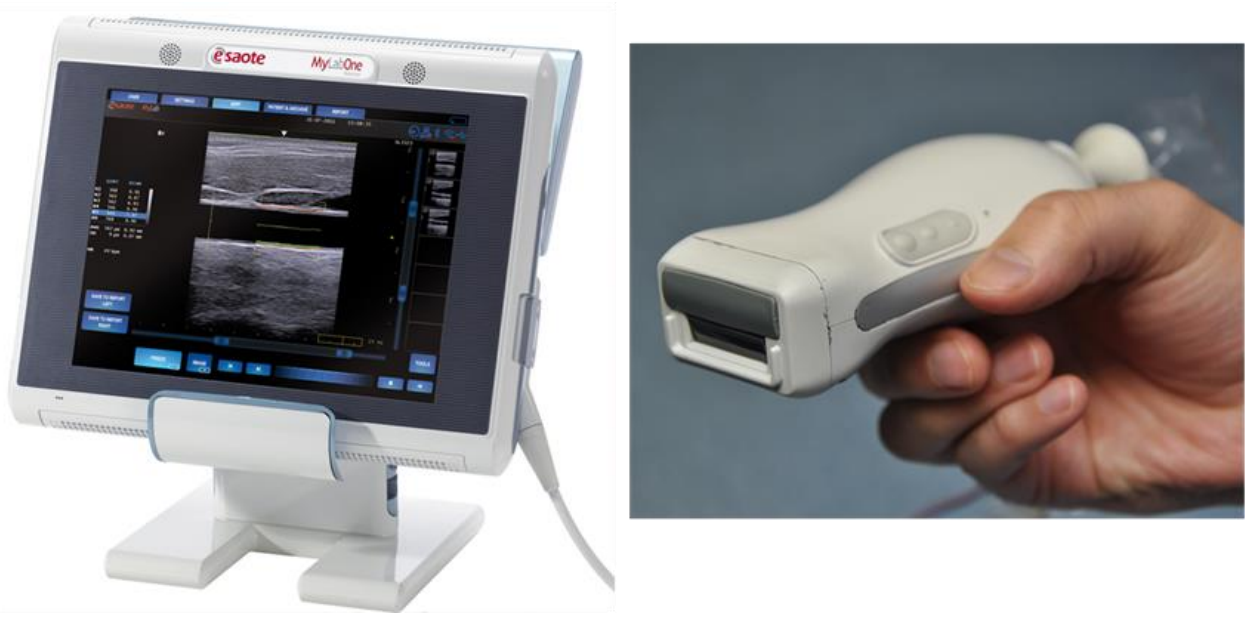

Figure 2.9 Portable system combining ultrasound and photoacoustics. Left is the US scanner and right shows the photograph of probe combining laser module and US transducer array.(Reprinted from ref. [36] Daoudi, K. et al. 2014. Optics Express, 22(21): p. 26365-26374. With permission from the authors and Optical Society of America.) 
This system was used to image a human proximal interphalangeal (PIP) joint and demonstrated detailed absorption distribution alongside the anatomical structure. Figure 2.10 shows combined PA/US images of the sagittal and transverse plane of the PIP. Skin, bone and joint gap are clearly visible in the US images. PA images with the complementary contrast depict the skin and blood vessel running parallel to the skin. The deeper PA signals correspond to the reflection of skin PA signal on bone. This work envisages a potential in visualizing rheumatoid arthritis using a point of care device.

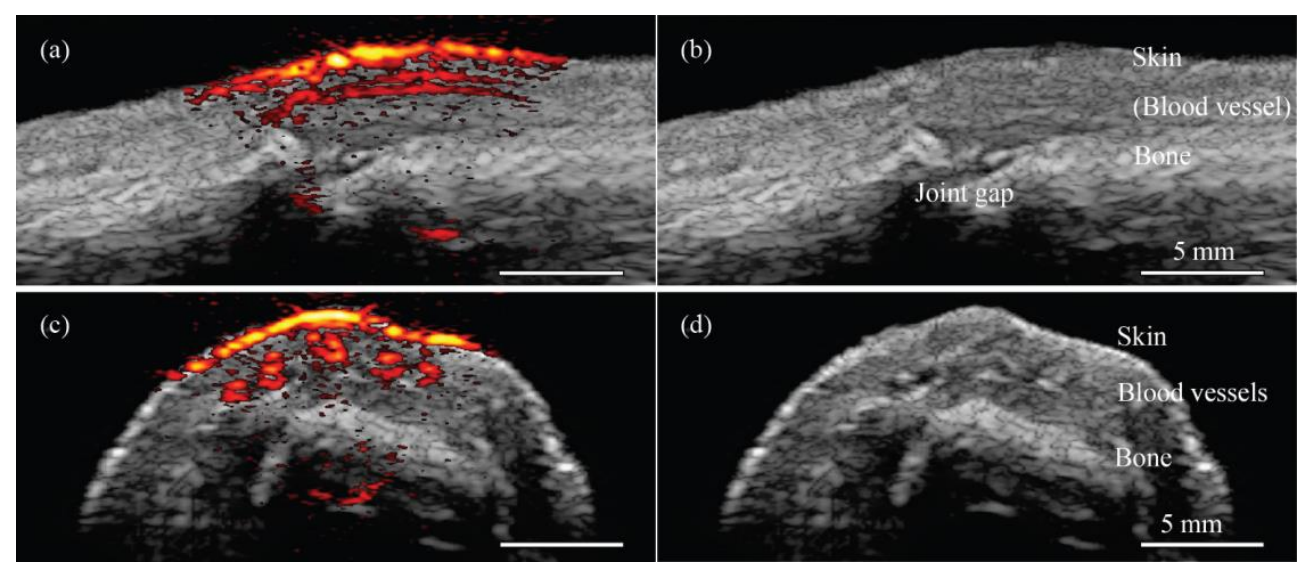

Figure 2.10 PA/US images of a human proximal interphalangeal joint in sagittal (a) and transverse (b) planes, with the upper part of the image corresponding to the dorsal side of the finger. On the right side, (b) and (c) show corresponding US only with anatomical structures indicated. (Reprinted from ref. [36] Daoudi, K. et al. 2014. Optics Express, 22(21): p. 26365-26374. With permission from the authors and Optical Society of America.)

\subsubsection{The Washington University in St. Louis system}

The Washington University group from St Louis in association with Philips research, North America developed a PA/US system from a commercially available US scanner [26, 28] (iU22, Philips Healthcare) which made use of a linear array probe with a bandwidth of 4-8 MHz (L8-4 Philips Healthcare). Figure 2.11 shows the photograph of the integrated set up.

The probe was physically integrated with a bifurcated optical fiber bundle (CB18043, Fiberguide) which was fed by a tunable dye laser (NS, Sirah) pumped by a Q-switched 
Nd:YAG laser (PRO-350-10, Newport). Laser pulses of $6.5 \mathrm{~ns}$ pulse duration were delivered for a radiant exposure of $3 \mathrm{~mJ} / \mathrm{cm}^{2}$ on the skin, well below the ANSI safety limit $\left(20 \mathrm{~mJ} / \mathrm{cm}^{2}\right)$.

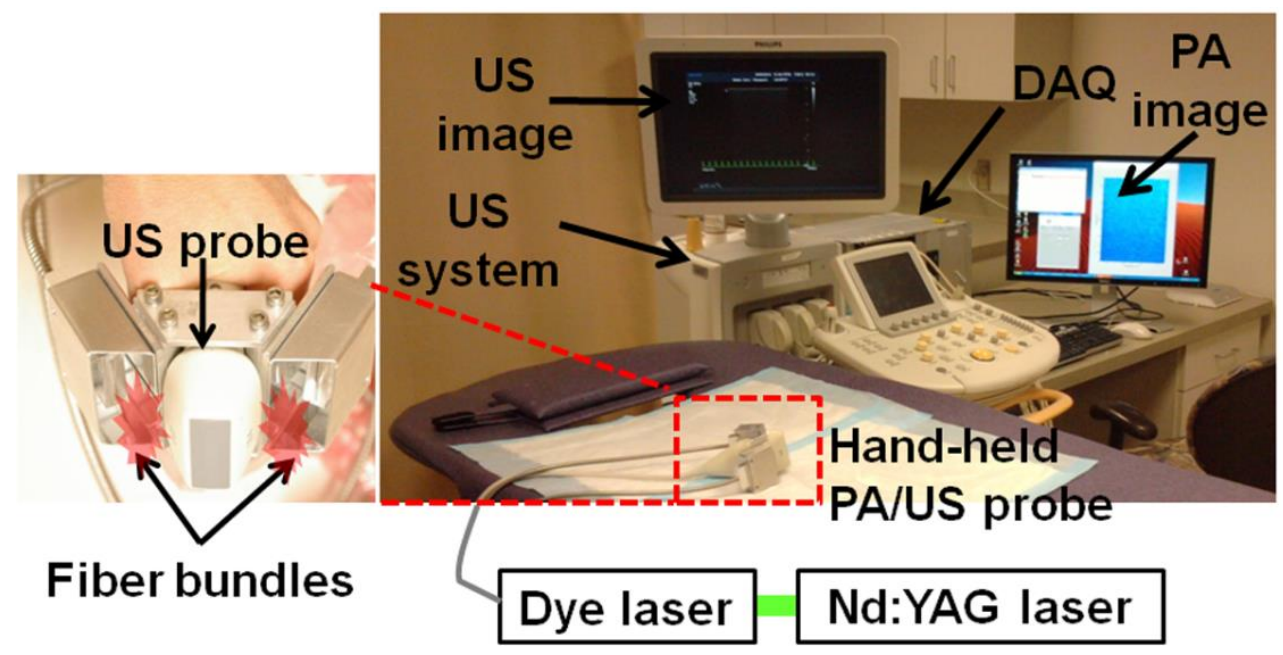

Figure 2.11 Photograph of the integrated PA and US imaging system modified from a clinical US array system. (Reprinted from ref. [26] Kim, C. et al. 2010.Biomedical Optics Express, 1(1): p. 278-284. With permission from the authors and Optical Society of America.)

The channel board in the system was modified in such a way that raw PA/US data were transferred to an external data acquisition system, which controlled the laser trigger signal, light wavelength tuning and post processing. The US images were displayed on the scanner's monitor, while the PA images, reconstructed with Fourier beam-forming, were displayed on a separate screen at $1 \mathrm{fps}$. The system was capable of frame rates up to $10 \mathrm{fps}$, but was limited due to the repetition rate of the laser $(10 \mathrm{~Hz})$ used.

This system proved to be effective for deep tissue imaging $(5 \mathrm{~cm})$ with a low radiant exposure $\left(1 / 7^{\text {th }}\right.$ of the ANSI safety limit). The use of a separate data acquisition system for PA data and processing makes this system relatively expensive. Further, the US and PA images were displayed on two different screens which has implications for comparison of images in real-time.

The system was used for PA image-guided identification and needle biopsy of sentinel lymph nodes (SLN) in pre-clinical work. Imaging the axillary region in a rat before and after 
methylene blue injection allowed the visualization of deeply located SLN (2 cm). Figure 2.12a shows the control PA B-scan image in which two deeply positioned blood vessels (3.2 and $3.5 \mathrm{~cm}$ ) are visible with excellent contrast. Figure $2.12 \mathrm{~b}$ shows the image captured 10 minutes after the injection of methylene blue. The uptake of methylene blue in the SLN could be monitored by following the image contrast of the SLN. The overlaid PA (jet scale) and US images (gray scale) shown in Figure 2.12c gives us clear morphological and functional information of methylene uptake in the SLN. The imaging depth attained without any signal averaging is comparable with the depths of axillary lymph nodes in humans $(<3 \mathrm{~cm})$, which shows its potential clinical utility.

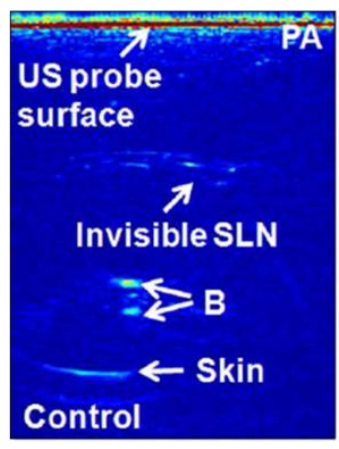

$1 \mathrm{~cm}$

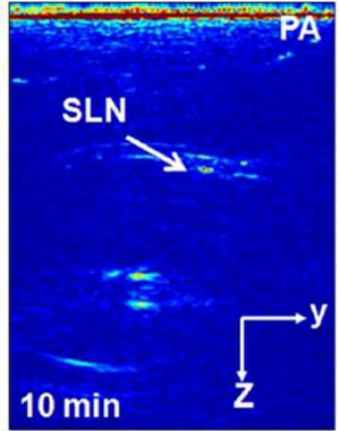

(b)
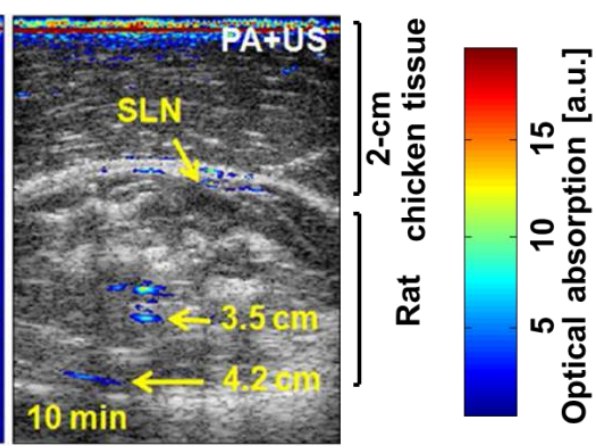

(c)

Figure 2.12 In vivo deeply penetrating PA imaging. (a) Control PA image acquired before methylene blue injection. (b) PA image taken 10 minutes after methylene blue injection. (c) Overlaid post-injection PA (pseudo color) and US (gray scale) images. B, blood and SLN, sentinel lymph node. (Reprinted from ref. [26] Kim, C. et al. 2010.Biomedical Optics Express, 1(1): p. 278-284. With permission from the authors and Optical Society of America.)

The authors also showed the feasibility of imaging a biopsy needle and methylene blue intake in SLN simultaneously (Figure 2.13). US imaging displayed a limited angular sensitivity for detecting a needle, as most of the incident acoustic energy is reflected away from the limited aperture US probe. Since the PA waves from the needle are cylindrical, these can be detected by the probe. PA and US image-guided SLN identification and needle biopsy demonstrated a promising substitute to current invasive axillary staging methods for breast 
cancer patients. This work also demonstrated the capability of PA imaging to image deep inside tissue $(5.2 \mathrm{~cm})$ with laser fluence well below the ANSI safety limit $\left(20 \mathrm{~mJ} / \mathrm{cm}^{2}\right)$.

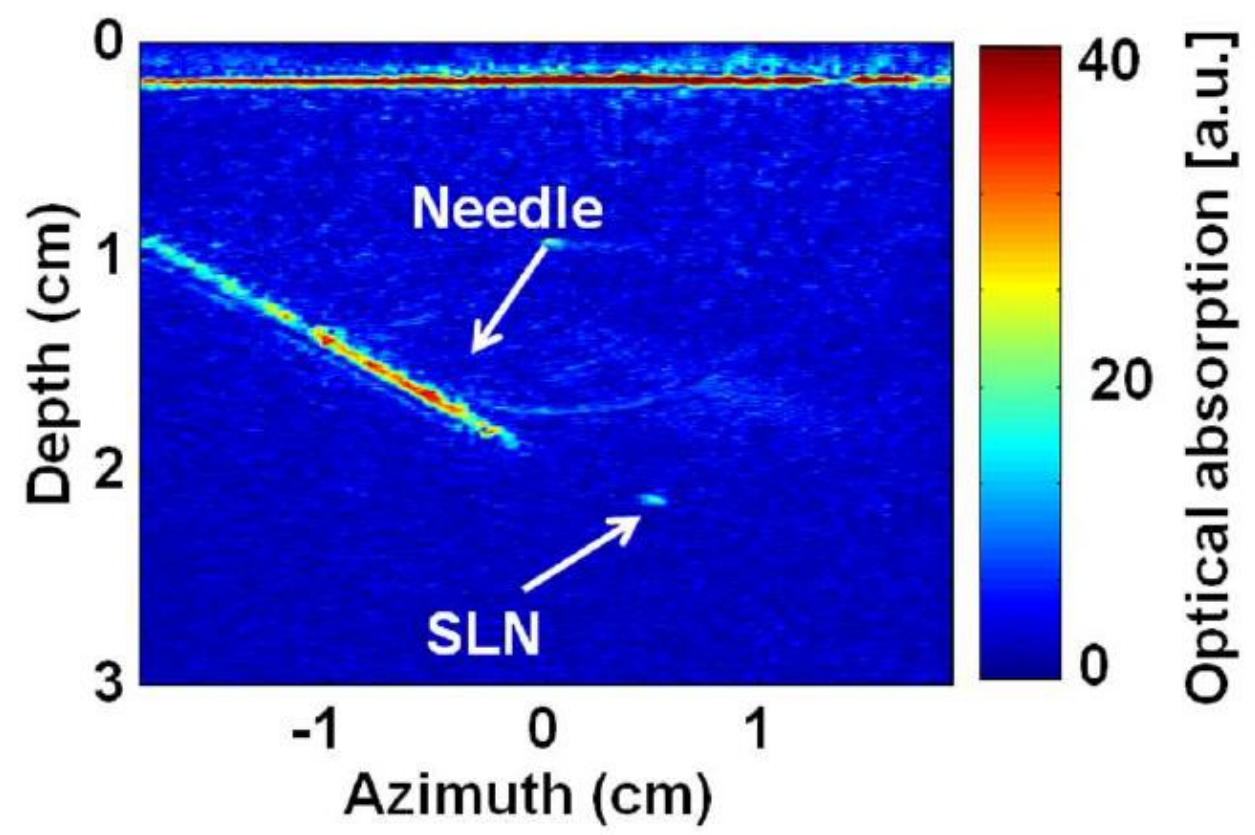

Figure 2.13 In vivo PA guidance of a metal needle (18-gauge). SLN, Sentinel lymph node. (Reprinted from ref. [26] Kim, C. et al. 2010.Biomedical Optics Express, 1(1): p. 278-284. With permission from the authors and Optical Society of America.)

\subsubsection{The TUM (Technical University Munich) system}

The group of Haisch at the Technical University Munich, developed an integrated US/PA set up (OPUS) derived from a commercial (General Electric) US scanner [37]. Figure 2.14 shows the schematic of the setup and US probe. A $100 \mathrm{~Hz}$ pulsed Nd:YAG laser pumping an OPO was synchronized with the US system that could switch between US and PA imaging. The bifurcated optical fiber ( $1 \mathrm{~mm}$ ) was connected side by side to the US probe with a clip, which allowed for changes in illumination angle. The probe used had 192 elements with a central frequency of $8.8 \mathrm{MHz}$ and a $-6 \mathrm{~dB}$ bandwidth of 4.1 MHz. Half the elements were used for PA and other half for conventional US. The data acquisition was done here sequentially for 96 elements at the laser repetition frequency $(100 \mathrm{~Hz})$, which resulted in a frame rate of 1.1 fps. This system proved the ability to use an integrated US/PA system for quantitative 
measurements by making use of spectroscopy. They also showed that the coupling angle between the laser light and the sample surface influences the depth where a maximum PA signal is generated.

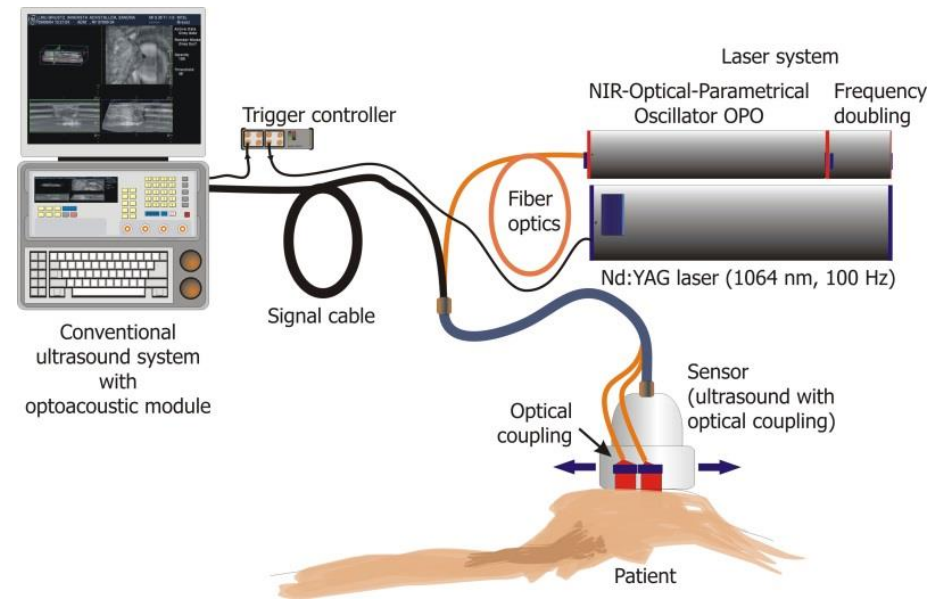

Figure 2.14 Schema of setup of OPUS system. The insert shows the US transducer with optical coupling. (Reprinted from ref. [37] Haisch, C. et al. Analytical and Bioanalytical Chemistry, 2010. 397(4): p. 1503-1510. With permission from the authors and Journal of Analytical and Bioanalytical Chemistry.)

This work also compared and tested four different reconstruction algorithms [Fouriertransform-based algorithm (FTA), Hough-transform-based algorithm (HTA), delay-and sum (DnS), and circular back-projection (CBP)] on the reconstruction speed, contrast and spatial resolution of the system. However low frame rate was a bottleneck for this system to do real time in-vivo measurements.

\subsubsection{Alpha prototype of Imagio system (Seno Medical Instruments, San Antonio, TX)}

Fronheiser et al [38] from Seno Medical Instruments and Fairway Medical Technologies reported a real-time laser opto-acoustic imaging system (LOIS) with the ability to image larger blood vessels deep inside the forearm with sufficient contrast and in real-time (10 fps). Figure 2.15 shows the photograph of the probe used. A Q-switched Nd:YAG laser with $6 \mathrm{~ns}$ pulses of $1064 \mathrm{~nm}$ at a repetition rate of $10 \mathrm{~Hz}$ was used. The light coupling was achieved 
using a bifurcated fiber with illumination on both sides of the probe (Figure 2.15). The output laser beam coupled into the 12-in. randomized fiber bundle (Fiberoptics Technology, Inc., Pomfret, Connecticut) bifurcates into two rectangular beams $(2 \times 50 \mathrm{~mm})$ to provide illumination with maximum radiant exposure of $15 \mathrm{~mJ} / \mathrm{cm}^{2}$. This system made use of a custom-made 128-element US probe with a center frequency of $5 \mathrm{MHz}$ and fractional bandwidth of $75 \%$ focused at $20 \mathrm{~mm}$ using an acoustic lens. PA data was acquired and by external data acquisition system with built in amplifiers and FPGAs (Field programmable Gate Arrays) for transfer to a PC. The PC-based real-time visualization of PA images was performed by custom-made LOIS-XP software that made use of 2D filtered back projection.

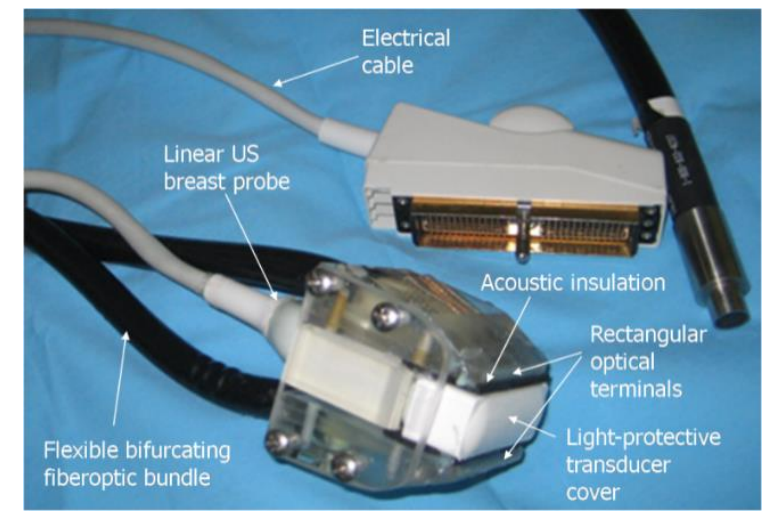

Figure 2.15 Photograph of LOIS probe. (Reprinted from ref. [38] Fronheiser, M.P. et al. Journal of Biomedical Optics, 2010. 15(2):021305. With permission from the authors and SPIE.)

The authors imaged blood vessels situated deep inside the forearm using their real time (10 fps) system. The PA data in Figure 2.16 (left) was sampled at $25 \mathrm{MHz}$ and reconstructed at a rate of $10 \mathrm{fps}$. The US image made at the same location is also shown at right. Analysis of the cross sectional diameter of ulnary artery at higher frame rates (10 fps) opened up the possibilities to measure blood flow using this system. Change in arterial diameter clearly indicated a cyclical increase and decrease in blood volume that likely reflects the arterial blood flow. This suggests that a real-time PA system can potentially be used, similarly to photoplethysmography, for monitoring blood flow. 

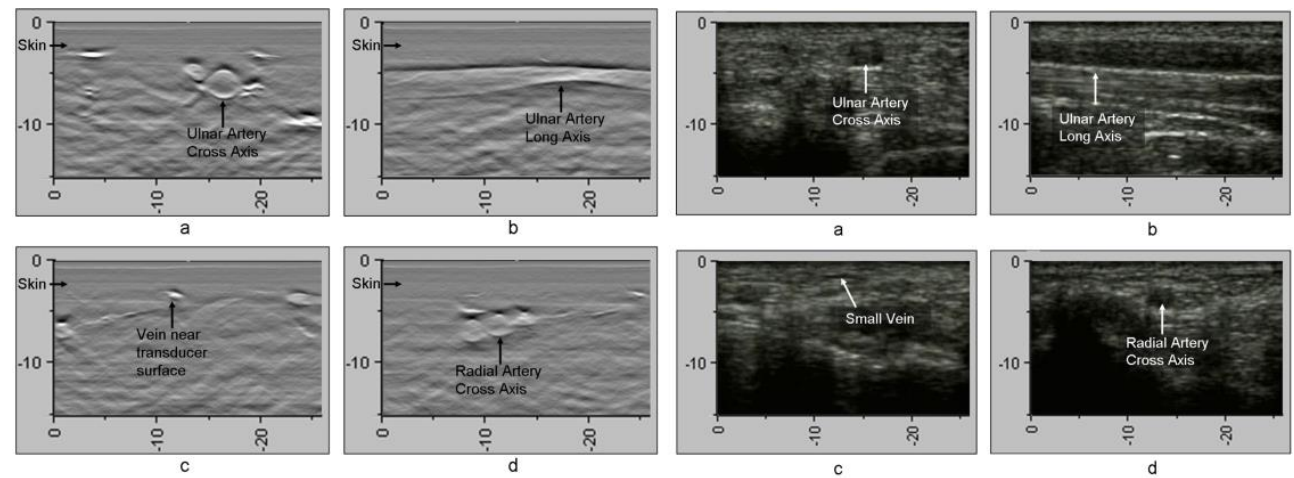

Figure 2.16 PA images (left) of the wrist vessels acquired in real-time during test 1: the ulnar artery in (a) a cross axis view and (b) a long axis view, (c) a vein near the surface, and (d) the radial artery. US images (right) acquired using probe manipulations identical to those yielding PA images (a) cross axis and (b) long axis views of the ulnar artery, (c) view of a vein in the mid-wrist region, and (d) the radial artery in cross axis view. (Reprinted from ref. [38] Fronheiser, M.P. et al. Journal of Biomedical Optics, 2010. 15(2): 021305. With permission from the authors and SPIE.)

\subsubsection{The University of Arizona system}

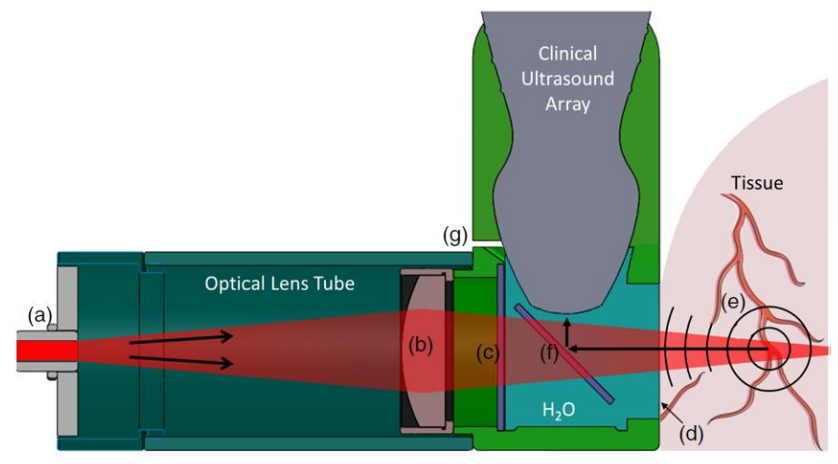

Figure 2.17 Cross-sectional view of the PA enabling device (PED) design as an attachment to a clinical US probe. (a) fiber optic connector, (b) Cylindrical focusing lens, (c) glass window, (d) thin transparent membrane, (e) optical absorbers, (f) reflecting glass plate, (g) drain hole. (Reprinted from ref. [39] Montilla, L.G. et al. Physics in Medicine and Biology, 2013. 58(1): p. N1-N12. With permission from the authors and IOP Publishing.) 
Appropriate light delivery has been an issue in PA imaging using commercial US scanners. In most of the systems explained above, light enters tissue at oblique angles and intersects the imaging plane in the tissue. This geometry confines the illumination pattern to specific depths, which is not optimal for imaging the entire region of interest. To address this issue, Montilla et al [39] came up with a solution called PA enabling device (PED) which can be hooked to any linear array US probe.

The approach exploits an optically transparent acoustic reflector to help direct laser illumination to the region of interest. PED was demonstrated in a system where the source is a pulsed laser coupled to an optical parametric oscillator (Surelite ${ }^{\mathrm{TM}} 532 \mathrm{~nm}$ I-20 and OPO plus; Continuum, Santa Clara, CA), producing wavelength tunable pulses at $20 \mathrm{~Hz}$. Light was coupled to the PED using an optical fiber (CeramOptec, East Longmeadow, MA). The US scanner used was a commercially available one (z.one Zonare Medical Systems) connected to a 64 elements linear array probe $(7.5 \mathrm{MHz})$. Frame rates of $20 \mathrm{fps}$ were achieved for PA imaging, which was limited by PRF of the laser. US imaging was done using planewave transmission and beam-forming. US and PA data from the scanner were transferred to a PC using Ethernet interface. Reconstruction was performed in MATLAB (Mathworks, Natick, MA) using delay and sum beam-forming considering one way (PA) or two-way propagation (US) of the acoustic waves. Figure 2.17 shows the mechanism of connecting PED to probes and how light illumination and collection of PA signals can be improved. Figure 2.18 shows the use of PED along with a linear array probe and a photograph of a live measurement.
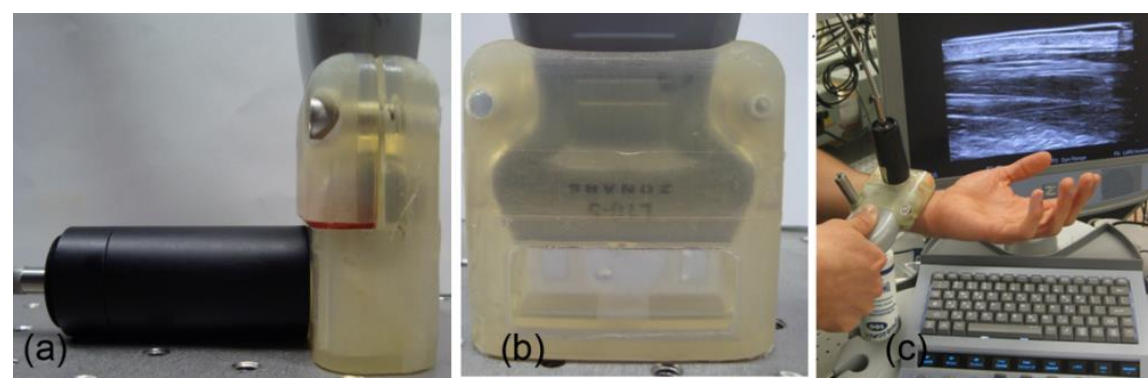

Figure 2.18 (a) and (b) Photograph of Liner array probe connected to PED (c) Photograph of the integrated system during imaging of wrist. (Adapted from ref. [39] Montilla, L.G. et al. Physics in Medicine and Biology, 2013. 58(1): p. N1-N12. With permission from the authors and IOP Publishing.) 


\subsubsection{The Helmholtz Zentrum / Technische Universität München systems - Ntziachristos group}

From the group of Ntziachristos at the Technical University Munich, Buehler et al [40] developed a real-time handheld multispectral optoacoustic imaging system (MSOT). Although this novel video-rate handheld system does not have US imaging capability (not reported), it is included in this review for showing the clinical relevance of multi-spectral PA imaging. Figure 2.19 shows the details of the handheld probe. This system used a fast laser compared to the previous MSOT system reported by the same group. The fast operation and switching of wavelengths helped in motion insensitive measurements, which is very crucial for systems based on handheld probes.

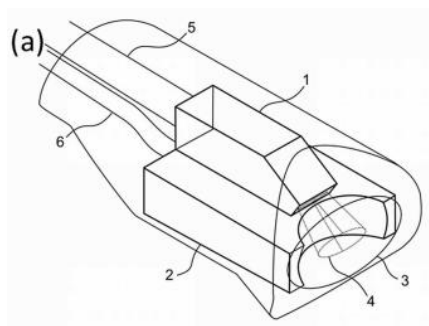

(c)

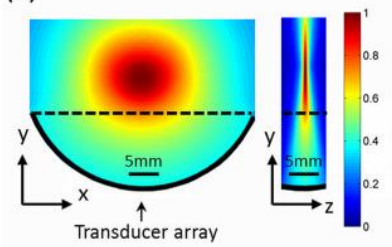

(b)

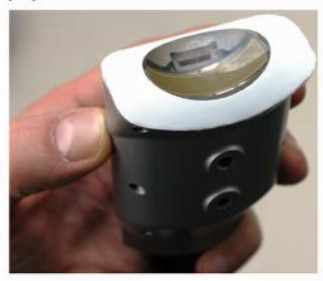

(d)

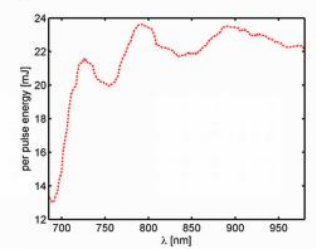

Figure 2.19 Drawing of the handheld device (a) and photograph of it (b). Simulated sensitivity distribution of the array within the detection band (c) and the wavelength dependency of the laser energy (d). 1, fiber bundle output; 2, transducer array; 3, membrane; 4, illumination pattern; 5 , fiber bundle, 6 , electrical cables.(Reprinted from ref. [40] Buehler, A. et al. 2013. Optics Letters, 38(9): p. 1404-1406. With permission from the authors and Optical Society of America.)

The US probe used was a custom made array of 128 elements with center frequency of 8 $\mathrm{MHz}$ and transmit-receive bandwidth of $60 \%$ manufactured using 1-3 piezo-composite technology. The elements in this probe were arranged in one row forming a spherical concave array. Light illumination was provided by a custom built laser system based on an OPO (680- 
$980 \mathrm{~nm}$ ) and pumped with a diode pumped Nd:YAG laser (InnoLas Laser GmbH, Krailing) which generated laser pulses with duration less than $10 \mathrm{~ns}$ and with a repetition rate of $50 \mathrm{~Hz}$ which helped in real time imaging. Light was coupled into a custom silica fused-end fiber bundle consisting of 256 fibers. At the output side of the bundle, the fibers were arranged in a rectangular aperture of dimensions $2 \mathrm{~mm} \times 2 \mathrm{~mm}$. It serves to create an illumination zone of $\sim 2.5 \mathrm{~mm}$ height and $\sim 12 \mathrm{~mm}$ width on the surface of the subject, coinciding with the US detection plane. The data acquisition system used here also was custom made which provided the capability of acquiring data from all 128 channels simultaneously. Signal processing, image reconstruction and spectral unmixing were performed on the GPU of the computer in real time. The coupling of tissue and probe was done in a novel way by making use of an acoustically transparent membrane, creating a cavity between it and the transducer array surface that was filled with water for good coupling of US. The authors also demonstrated the use of a model based inversion technique for better reconstruction of PA images.
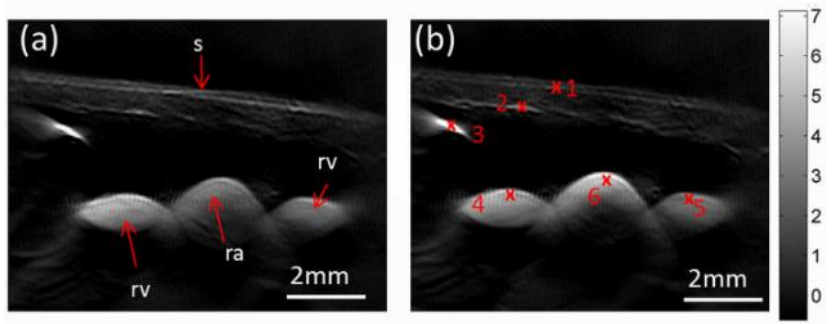

(c)

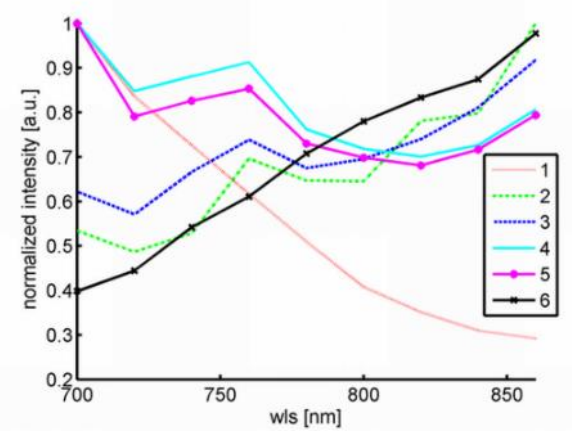

Figure 2.20 (a) PA image of the wrist at $760 \mathrm{~nm}$ and (b) $860 \mathrm{~nm}$. rv, radial vein; ra, radial artery; s, skin. (c) Profiles through the multispectral dataset from the locations labeled in (b). (Reprinted from ref. [40] Buehler, A. et al. 2013. Optics Letters, 38(9): p. 1404-1406. With permission from the authors and Optical Society of America.) 
This handheld Multispectral optoacoustic Tomography (MSOT) system was used to demonstrate the capability of differentiating between arteries, veins, and melanin based on their known absorption spectra. Figure 2.20a and b show the PA image of the left wrist of a healthy volunteer with wavelength $760 \mathrm{~nm}$ and $860 \mathrm{~nm}$ respectively. Figure $2.20 \mathrm{c}$ shows the spectral profiles of the marked points in Figure 2.20b. Spectrum 1 strongly resembles the absorption spectrum of melanin; spectra 2, 3, 4, and 5 resemble deoxyhemoglobin with its characteristic peak at $760 \mathrm{~nm}$, and spectrum 6 resembles the absorption of oxyhemoglobin.

The authors also demonstrated dynamic imaging by obstructing and releasing the blood flow to the finger of a healthy volunteer and monitoring the changes using multispectral PA imaging. By this, they were able to extract temporal evolution of oxy and deoxyhemoglobin, total blood volume $\left(\mathrm{BV}=[\mathrm{Hb}]+\left[\mathrm{HbO}_{2}\right]\right)$ and the oxygen saturation $\left(\mathrm{SaO} 2=\left[\mathrm{HbO}_{2}\right] / \mathrm{BV}\right)$ dynamically.
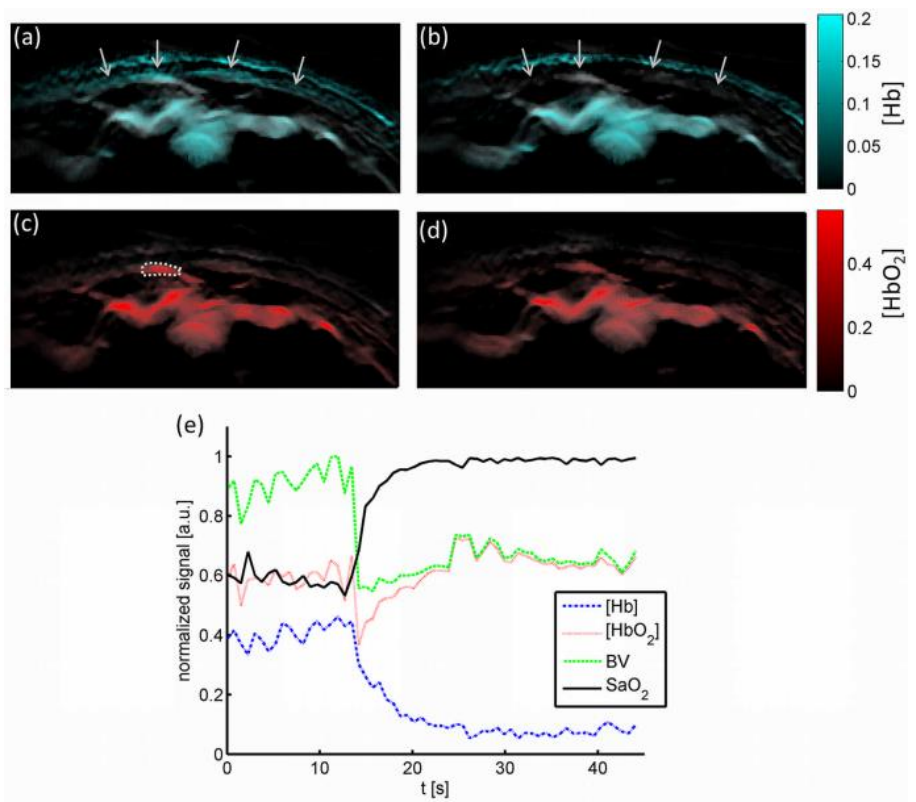

Figure 2.21 (a)-(d) Unmixed deoxyhemoglobin $(\mathrm{Hb})$ and oxyhemoglobin $\left(\mathrm{HbO}_{2}\right)$ superimposed onto the total BV image before (first column) and after (second column) release of vascular blocking. (e) Temporal evolution of the extracted properties within the area indicated in (c). The arrows indicate the area at which the deoxyhemoglobin signal changes. (Reprinted from ref. [40] Buehler, A. et al. 2013. Optics Letters, 38(9): p. 1404-1406. With permission from the authors and Optical Society of America.) 
Figure 2.21a and b shows the unmixed deoxyhemoglobin signal, superimposed onto the total BV image, 10 seconds before (Figure 2.21a) and 15 seconds after (Figure 2.21b) release of the rubber band used for obstructing blood flow. Figure 2.21c and $\mathrm{d}$ show the corresponding oxyhemoglobin signal. Figure 2.21e depicts the temporal evolution within the area marked in Figure $2.21 \mathrm{c}$ during the measurement time. The accumulation of deoxygenated hemoglobin, when the blood flow was obstructed is evidently visible as higher signal just below the skin (Figure 2.21a). This signal got diminished once the blood flow was normal (Figure 2.21b).

Dima et al [41] from the same group reported dual mode PA/Duplex US (DUS) measurements of the carotid artery at the peak of volumetric expansion caused by heartbeat in a healthy volunteer. DUS is a combination of conventional anatomical US and color Doppler US. This work makes use of a different system with custom made curved array US probe (64 elements, $5 \mathrm{MHz}$ center frequency).
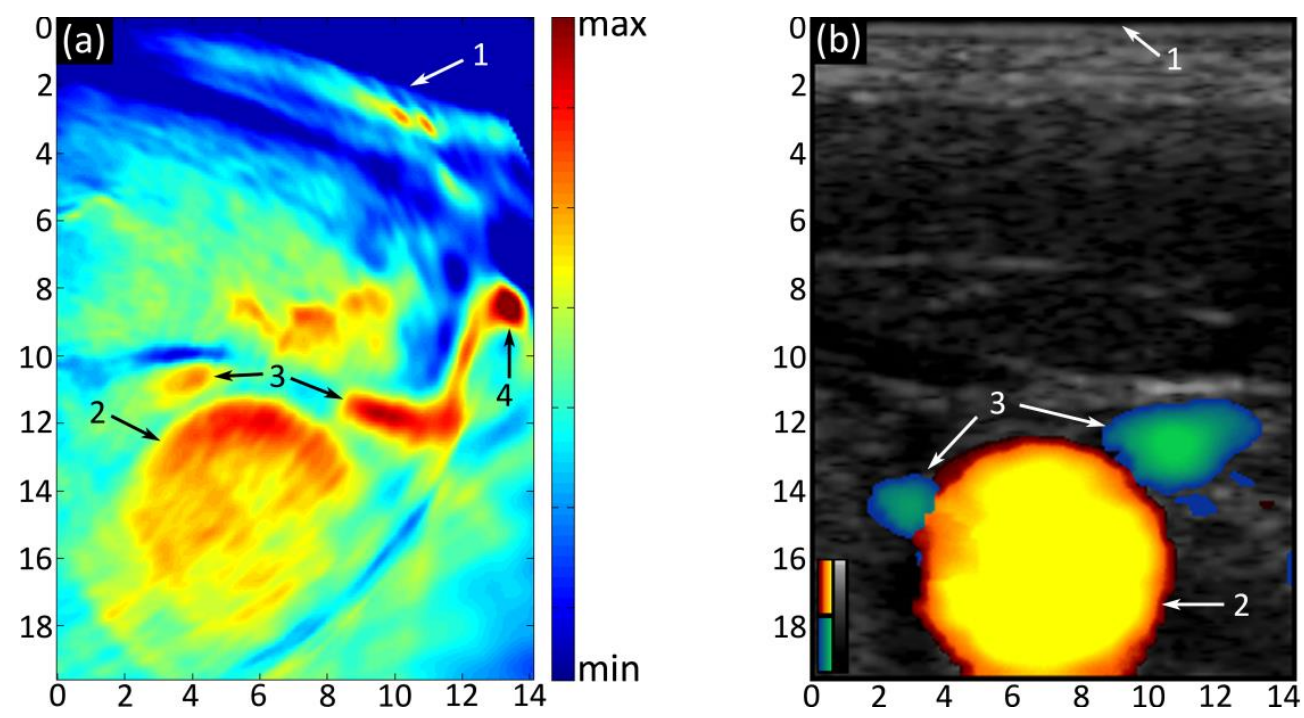

Figure 2.22 Carotid Imaging Using Photoacoustics and Duplex US. (a) PA Image, (b) Directional DUS image indicating positive flow in yellow/red; negative flow in green/blue. Annotations: 1. Skin surface, 2. Common carotid, 3. internal jugular vein, 4. External jugular vein. (Reprinted from ref. [41] Dima, A. and V. Ntziachristos. 2012 Optics Express, 20(22): p. 25044-25057. With permission from the authors and Optical Society of America.) 
Figure 2.22a shows the PA image acquired at a wavelength of $800 \mathrm{~nm}$. The common carotid artery is visualized (marked as 2) clearly as a round structure from depth of 12 to 18 $\mathrm{mm}$. The skin (marked as 1), the right internal jugular vein (marked as 3 ) and an external jugular vein (marked as 4) are also visible in the image. To validate the findings, DUS of the same location was also performed (Figure 2.22b). The color maps used (positive flow: yellow/red; negative flow: green/blue) simplify identification of the carotid artery and vein. The linear array used for DUS measurement here flattened the skin and pushed the external jugular vein out of the imaging window. Because of less pressure applied when holding the probe, the common carotid is seen at slightly a different depth $(13-19 \mathrm{~mm})$. The right internal jugular is also visible in the DUS image. This demonstrated that PA imaging could be a good complementary technique along with DUS for imaging Carotid and surrounding vascular structures.

\subsubsection{The Helmholtz Zentrum / Technische Universität München systems - Razansky group}
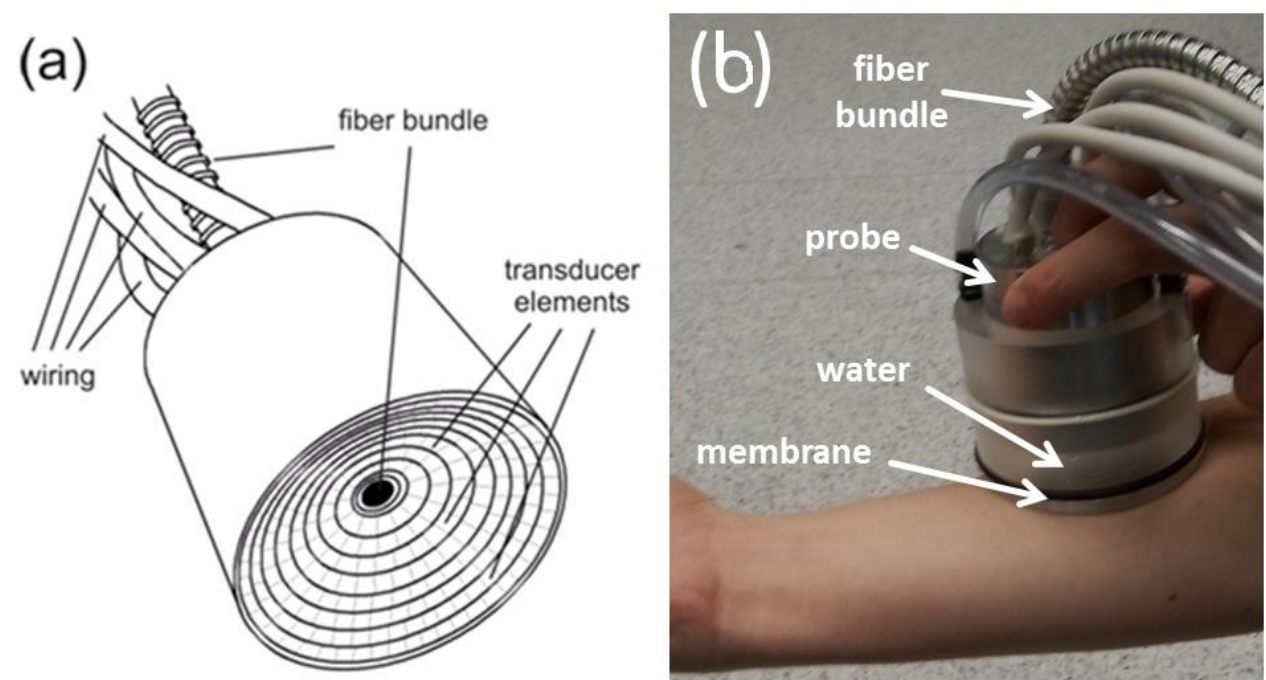

Figure 2.23 (a) Layout of the probe showing the arrangement of transducer elements and fiber bundle, (b) Photograph of the probe during measurement. (Reprinted from ref. [42] X.L. Dean-Ben, and D. Razansky 2013. Photoacoustics, 1(2013): p. 68-73. With permission from the authors and Elsevier.) 
From the group of Razansky at the Technical University Munich, Xose Luis Dean-Ben et al [42] reported on the development of a portable clinical system for three dimensional PA visualization of deep human tissues at video rate. Three-dimensional PA imaging and spectroscopy using a handheld probe, without any cumbersome scanning stage was a novelty. This system without US imaging capability (not reported) is included in this review because of its innovative probe design and capabilities. This vMSOT(Volumetric Multi-spectral optoacoustic tomography) device used a custom made two-dimensional array of 256 ultrasonic detectors (Imasonic SaS Voray, France) arranged upon a spherical surface with a radius of $40 \mathrm{~mm}$ and covering a solid angle of 90 degrees. Elements had a center frequency of $4 \mathrm{MHz}$ and a bandwidth of $100 \%$. Figure 2.23a and b show the layout and photograph of the probe respectively. The elements were manufactured using piezo-composite material for better sensitivity. This is important for real time imaging as improving SNRs by averaging is not the best option.

The arrangement of transducer elements here helped in allowing a full tomographic access to the area of interest by collection of signals from broad angles. The excitation light from a pulsed laser was delivered to the object through a silica fused-end fiber bundle (CeramOptics GmbH, Bonn, Germany) inserted into a hole located in the center of detection array as shown in Figure 2.23a. A wavelength tunable (690-900 nm) optical parametric oscillator (OPO) laser (Phocus, Opotek Inc; Carlsbad, CA) generating $10 \mathrm{~ns}$ pulses at a repetition rate of $10 \mathrm{~Hz}$ was used. Raw data from all elements were simultaneously sampled at $40 \mathrm{MHz}$ and using a custom made data acquisition system (Falkenstein Mikrosysteme $\mathrm{GmbH}$, Taufkirchen, Germany). The acquired data were then reconstructed on a workstation computer 2x Intel Xeon DP X5650 $(6 \times 2.67 \mathrm{GHz})$ with $144 \mathrm{~GB}$ of RAM. Since one laser pulse could produce complete volumetric data sets from imaged object, 3D real time imaging was possible with this system.

The system while claiming excellent resolutions with many capabilities uses custommade components such as the US probe, data acquisition system, computer and the laser, which would make it quite expensive. The repetition rate of the laser $(10 \mathrm{~Hz})$ limited the time resolution of this system. 


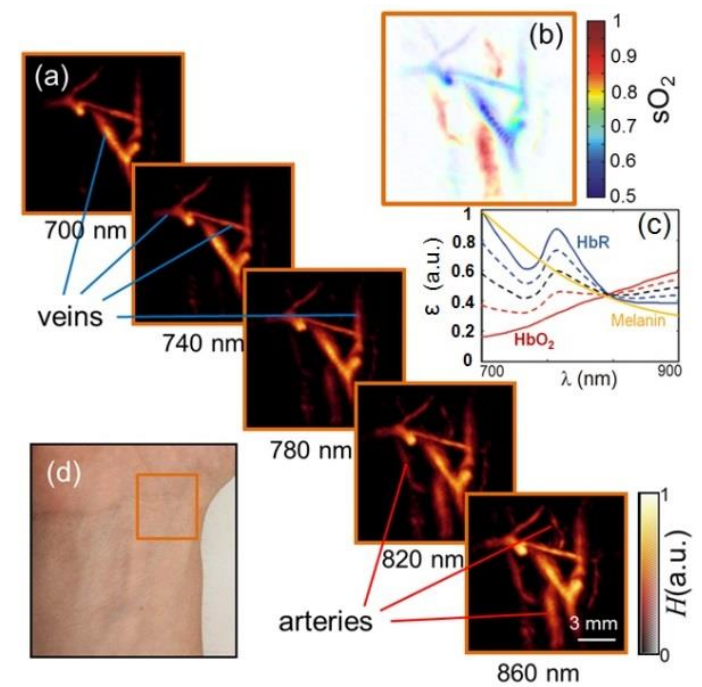

Figure 2.24 Multispectral tomographic reconstructions of the wrist region. (a) Volumetric images acquired at 5 different wavelengths in the near infrared. (b) Map of blood oxygenation saturation as calculated from images acquired at different wavelengths. (c) Absorption spectra of major tissue chromophores in arbitrary units. (d) Color photograph of the imaged region. (Reprinted from ref. [42] X.L. Dean-Ben, and D. Razansky 2013. Photoacoustics, 1(2013): p. 68-73. With permission from the authors and Elsevier.)

The authors demonstrated high resolution volumetric multispectral PA tomography for real-time visualization of blood oxygenation parameters. This work claimed the capability to do three dimensional real time imaging with the handheld probe, which is evidently difficult to achieve using other imaging devices.

Figure 2.24 shows the results of multispectral tomographic imaging of the wrist of a healthy volunteer. It is clear from Figure 2.24a that pixel intensity increases with wavelength for some of the vessels (Arteries) and it remains more or less constant for other vessels (Veins). Strongest background signal was observed for $700 \mathrm{~nm}$ as melanin absorbs more at this wavelength. By applying a spectral unmixing algorithm, map of blood oxygenation (Figure 2.24b) accurately revealed the location of veins and arteries. This work has good potential as a clinical tool, particularly in applications related to cardio vascular diseases, where blood oxygenation and delivery are diagnostically critical. 


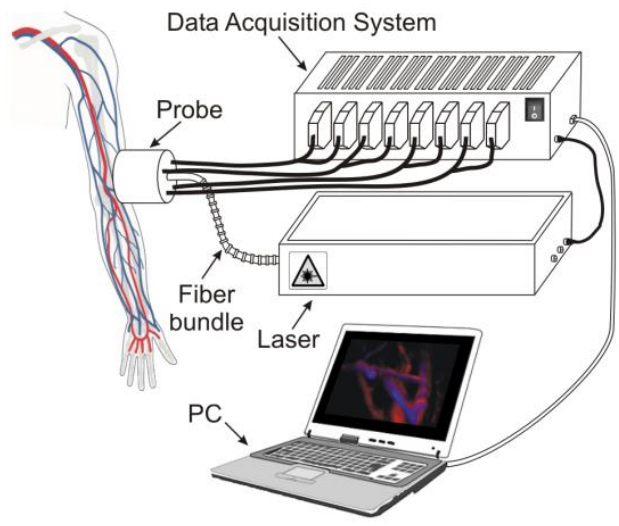

Figure 2.25 Schematics of the real-time handheld optoacoustic imager. (Reprinted from ref. [43] X.L. Dean-Ben, and D. Razansky. 2014. Nature, Light: Science \& Applications, 3:e137. With permission from the authors and Nature Publishing Group.)

The same group recently reported an improved version of this system which used a fast laser with fast wavelength tuning which in turn provide volumetric spectrally enriched tomography in real-time [43]. Figure 2.25 shows the schematic representation of this handheld PA-imaging scanner. This is the first reported system which delivers feasibility of PA imaging in 5 dimensions, i.e., rendering volumetric images of spectrally resolved PAc data in real time. The US array used was the same as in their previous system [42]. The illumination source was a custom-made optical parametric oscillator-based laser, generating $5 \mathrm{~ns}$ duration pulses with energy of up to $20 \mathrm{~mJ}$ at a pulse repetition rate of up to $50 \mathrm{~Hz}$. A dedicated tuning system based on fast mechanical rotation of the optical parametric oscillator crystal had been developed that allowed changing the wavelength to any value between 700 and $900 \mathrm{~nm}$ on a per-pulse basis. In this way, only $100 \mathrm{~ms}$ was required to acquire an entire volumetric multispectral dataset at five different wavelengths. The acquired data was then transferred to a personal computer using Ethernet port and GPU based processing was done. A 3D back projection reconstruction algorithm with spectral un-mixing for spectral analysis, could be performed by the GPU enabled system in less than $20 \mathrm{~ms}$. The fast acquisition, switching of wavelengths and processing helped to provide truly real time multispectral tomographic imaging. This state of the art system opens new prospects with several important clinical applications envisioned in clinical practice as well as in pre-clinical research. The 
only limitation of this system to enter the clinics would be the bulky laser used for the purpose.

\subsubsection{The VisualSonics system}

Needles et al [44] reported on the development and initial application of a fully integrated PA micro-US system, which offered co-registered US and PA imaging with options for spectral measurements. Figure 2.26 shows the schematic diagram of the prototype PA imaging system (Vevo LAZR, VisualSonics, Toronto, ON, Canada) developed by them.

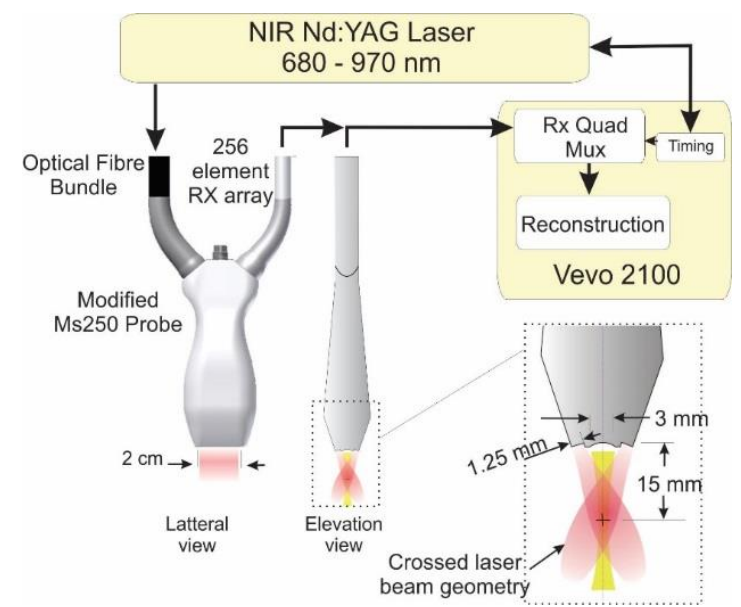

Figure 2.26 Schematic diagram of the LAZR PA imaging system showing (left) the integrated transducer array and the block diagram of the signal processing chain. Separate cable/fiber-optic bundles are used to excite optical and or acoustic elements. (right). (Reprinted from ref. [44] Needles, A. et al. 2013. IEEE Transactions on Ultrasonics, Ferroelectrics and Frequency Control, 60(5): p. 888-897. With permission from the authors and IEEE.)

The US probe used in this system was a linear array probe (MS250, VisualSonics Inc) having 256 elements with a center frequency of $21 \mathrm{MHz}$. Light illumination was provided by a tunable (680 to $970 \mathrm{~nm}$ ) NIR Nd:YAG laser with a repetition rate of $20 \mathrm{~Hz}$. The light was coupled using fiber optic bundles to the special housing where the US probe was fixed in such a way that crossed laser beam geometry at around $15 \mathrm{~mm}$ deep was achieved as shown in Figure 2.26. On receive, signals from 4 blocks of 64 elements (256 total) were transferred back to the Vevo 2100 scanner for reconstruction of a full-frame PA image. The laser 
repetition rate used here was $20 \mathrm{~Hz}$, yielding a PA imaging rate of $5 \mathrm{~Hz}$. Screen interfaces allowed the user to select wavelengths in the 680 to $970 \mathrm{~nm}$ range or to select an oxygenation mode in which sequential PA frames were acquired at 750 and $850 \mathrm{~nm}$ to calculate total hemoglobin and oxygen saturation. Three-dimensional data were acquired by scanning the transducer using a linear stepper motor during 2D PA measurements.

The system is intended predominantly for pre-clinical work such as visualizing and monitoring vasculature associated with cancer growth in small animals such as mice. Clinical applications are not impossible with the instrument, but with the present high-frequency US probe these will be restricted to superficial imaging applications. The laser used here was again bulky in the present version.

Langhout et al [45] used this system from Visualsonics and showed the viability of using PA imaging to identify melanoma metastasis in human lymph nodes. Multispectral PA Imaging with spectral unmixing was employed for required contrast. High-resolution US imaging was also done for orientation and identification of structures.
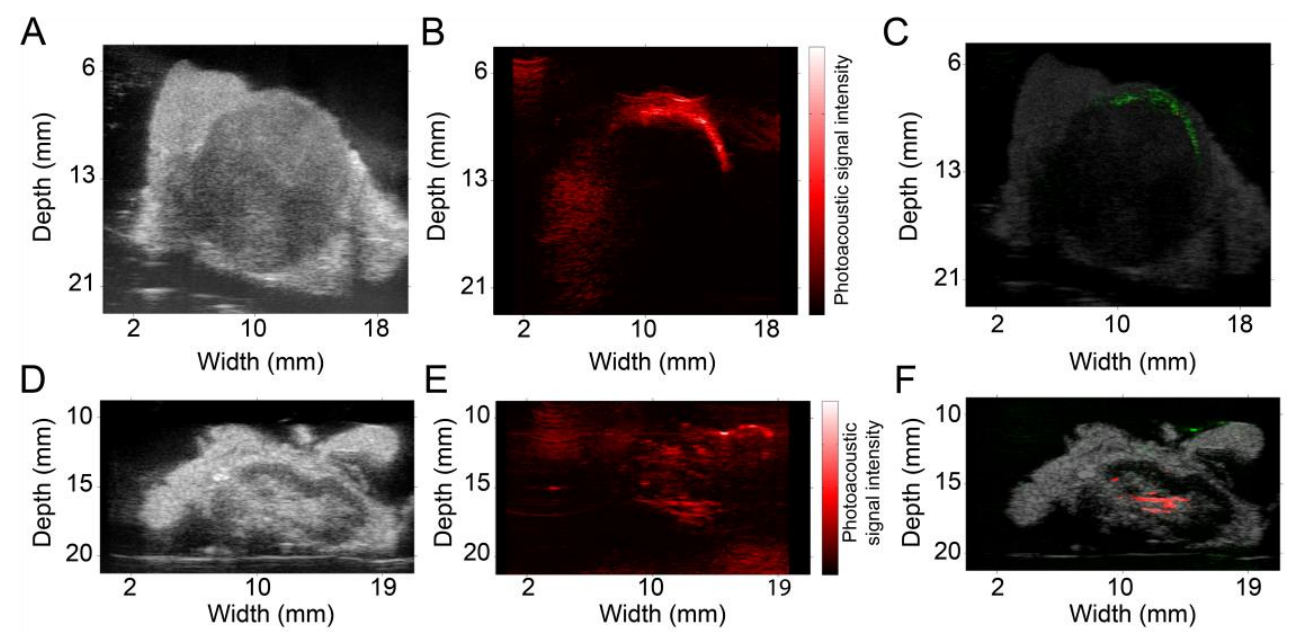

Figure 2.27 PA Imaging (Ex-Vivo) of human Lymph Nodes: Tumor positive node images (A, B, C), Benign node images (D, E, F); A, D) US Image, B, E) PA Image, C, F) Spectrally Unmixed PA Image superimposed on US Image. (Adapted from ref. [45] Langhout, G.C. et al . 2014. International Journal of Biomedical Imaging. 2014, 163652. With permission from the authors and Hindawi Publishing Corporation.) 
This work was able to differentiate optical absorption by melanin and hemoglobin in resected human lymph nodes by careful selection of light wavelength. Figure 2.27A shows the US image of a tumor positive lymph node. Here the node is seen as a round structure, surrounded by hyperechogenic fat tissues. The corresponding PA image (Figure 2.27B) shows strong surface signals, while deeper within the node signals are absent. Spectral unmixing was performed to confirm the reason for the high contrast region in PA image. The resulting image superimposed on the US image is shown in Figure $2.27 \mathrm{C}$, where the green region represents pixels with spectral resemblance to melanin. The presence of the high absorbing melanin deposits at the surface limited light penetration to a few mm leading to the lack of signal from deeper within the node. There appear no traces of blood contrast from the spectral unmixing in this region, and this observation is validated from histopathological findings.

Figure 2.27D shows the US image of a benign lymph node. This shows a bean shaped node with a brighter hilum enclosed by bright fatty tissue. The PA image (Figure 2.27E) displays vessel-shaped structures at $16 \mathrm{~mm}$ depth and signal at the surface of the fatty tissue. Spectral unmixing and analysis (Figure 2.27F) indicate that the vessels shaped structures contain blood, while the signal from the surface of the fatty regions does not resemble either the blood or melanin reference spectra. This work shows the relevance of combining the two modalities, with a complete anatomical visualization of the node using US, combined with the spectroscopic molecular selectivity possible with PA.

\subsubsection{The University of Michigan systems}

From the group of X. Wang, Yuan et al [46] reported on a real-time integrated US/PA system in which back-projection (BP) algorithm for PA image reconstruction was optimized to reduce the computational cost and facilitate parallel computation on a state of the art graphics processing unit (GPU) card. For building this system, the Verasonics US Data Acquisition platform (Model V3, Redmond, WA) was programmed to work along with the multicore computer equipped with the NVidia GeForce GTX690 GPU card. The US probe used here was a linear array probe (CL15-7, Philips North America Corporation, Andover, MA) with 128 elements, $11.25 \mathrm{MHz}$ center frequency, and a 75\% -6 dB bandwidth. The illumination was provided by a tunable optical parametric oscillator (OPO) (Vibrant B, Opotek inc; Carlsbad, CA) pumped by the second harmonic output of a Nd:YAG pulsed laser (Brilliant 
B, Quantel, Bozeman, MT). Laser pulse duration and pulse repetition frequency are $5.5 \mathrm{~ns}$ and $10 \mathrm{~Hz}$ respectively. By switching the transmission on and off, exactly synchronized with the laser pulses, this system employed the PA and US modes alternatively, both at a frame rate of $10 \mathrm{~Hz}$. The PA and US measurements were done alternatively and displayed on the same screen at a rate of $10 \mathrm{fps}$. US image processing and reconstruction was performed on the multicore CPU, while PA image reconstruction was completely done on the GPU, avoiding conflicts between processing and display of the two imaging modes. Figure 2.28 shows the schematic of the system.

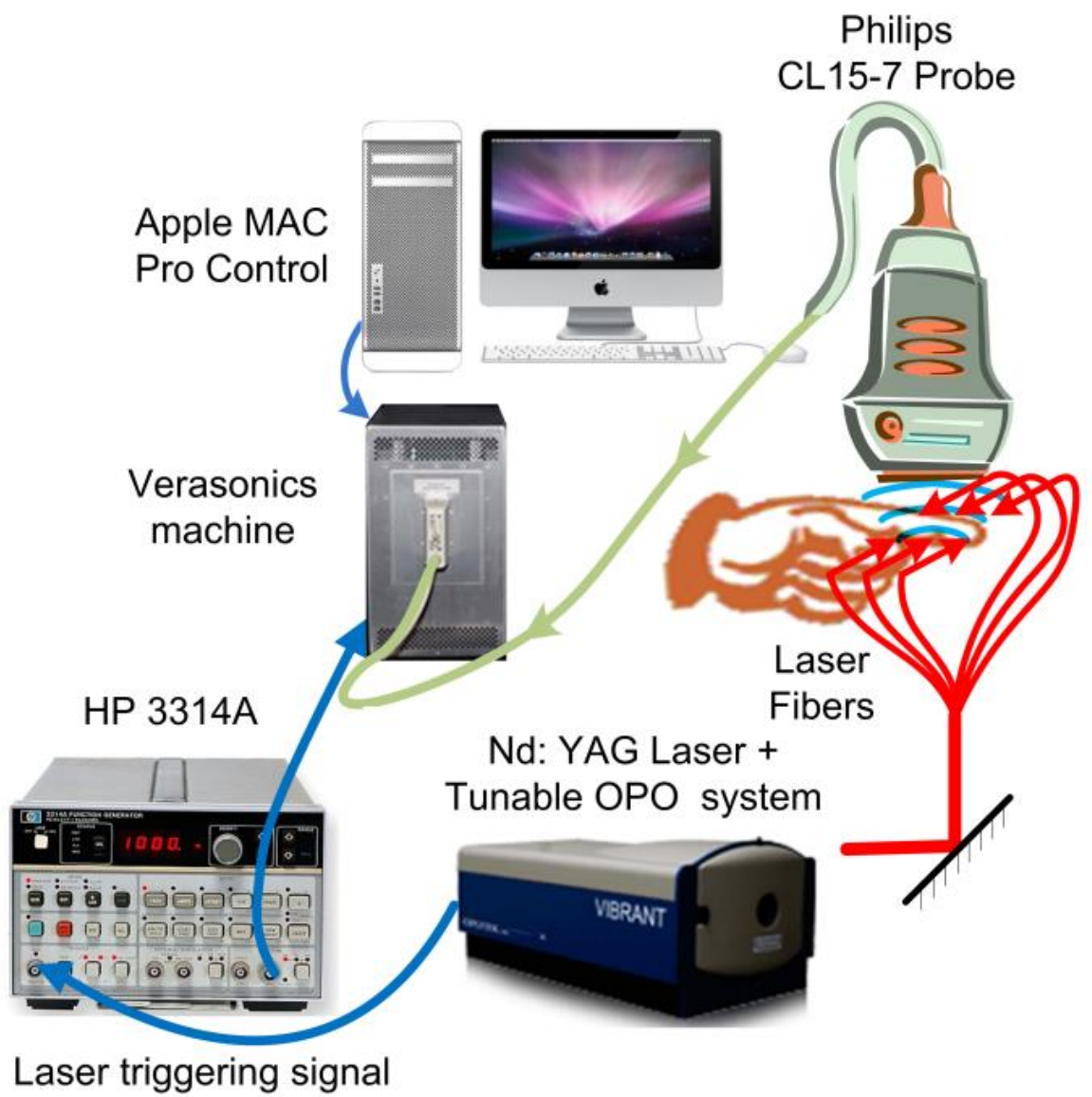

Figure 2.28 Schematic diagram of the real-time US/PA system. (Reprinted from ref. [46] Yuan, J. et al. 2013. Journal of Biomedical Optics, 18(8):086001. With permission from the authors and SPIE.) 
In PA imaging mode, when the image size was $1024 \times 512$ pixels and the data volume of signals was 128 channels by 2048 points, the maximum frame rate achieved with this system was $17 \mathrm{fps}$, sufficient for visually continuous real-time imaging. This real time US/PA system was found to be promising for clinical settings except for the fact that it makes use of a bulky water-cooled Q-switched laser system.
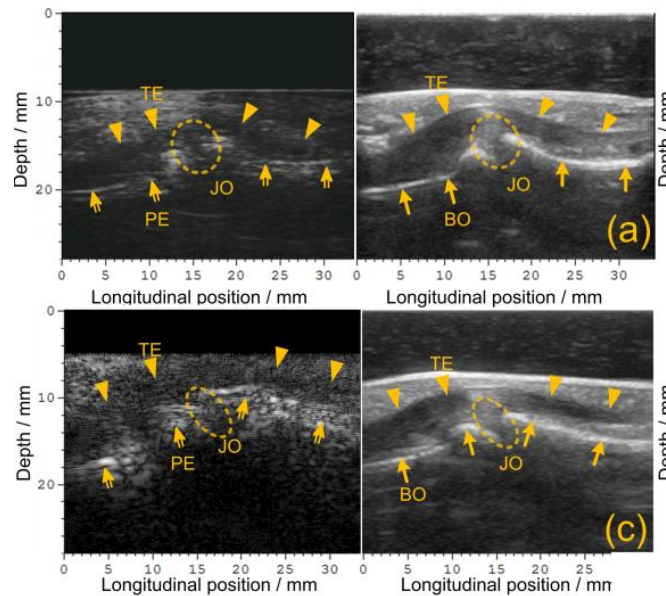
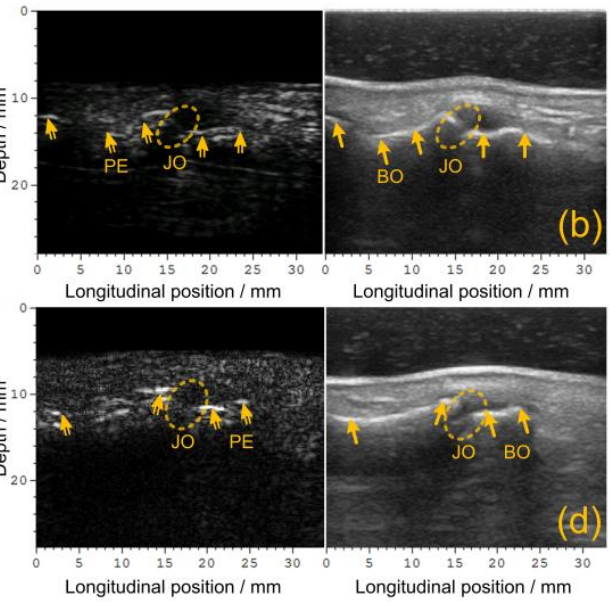

Figure 2.29 PA (left in each subfigure) and US (right in each subfigure) images acquired from healthy volunteers. (a) Coronal plane [volar] and (b) sagittal plane images acquired from the PIP joint of an index finger of a male volunteer; (c) Coronal plane [volar] and (d) sagittal plane images acquired from the PIP joint of an index finger of a female volunteer. TE: tendon, JO: joint, PE: periosteum, BO: bone. (Reprinted from ref. [47] Xu, G., et al. 2013. Journal of Biomedical Optics, 18(1):010502. With permission from the authors and SPIE.)

$\mathrm{Xu}$ et al [47] from the same group imaged finger joints with a slightly different version [not real-time] of PA/US system and demonstrated the ability to recover comparable features using both contrasts in healthy finger joints being able to depict the osseous structures, as well as tendon and cartilage (Figure 2.29). However, the US images represent backscattered acoustic energy from bony structures, while PA images most likely represent optical absorption in the vasculature at the bone surfaces. For the first time, it was shown that the tendon could be distinguished compared to the surrounding soft tissue in PA images primarily based on the higher blood content in the latter. This work shows that NIR light penetration is 
sufficiently deep for PA B-scan imaging of finger joints, which may have potential in visualization of inflammation in rheumatoid arthritis.

\subsubsection{The acoustic delay- line system}

Cho et al $[48,49]$ recently reported on a handheld PA tomography probe built using acoustic delay lines. Figure $2.30 \mathrm{~b}$ shows the basic principle employed in this system in comparison with the traditional systems (Figure 2.30a). This system was not capable of US imaging, but the technique used is worth reviewing considering the novelty in it, which envisages miniaturized systems for PA tomography. The typical propagation time of a PA signal in the tissue is in the range of microseconds $(\mu \mathrm{s})$, while the time between generation of two PA signals is much longer, typically of the order of milliseconds (ms), depending on the laser repetition rate $(10-20 \mathrm{~Hz})$. The system proposed here made use of this knowledge to delay the PA signals from different channels and acquire using two transducers instead of an array. This simplified the set up and reduces the overhead of using data acquisition systems that could handle multiple channels at a time.
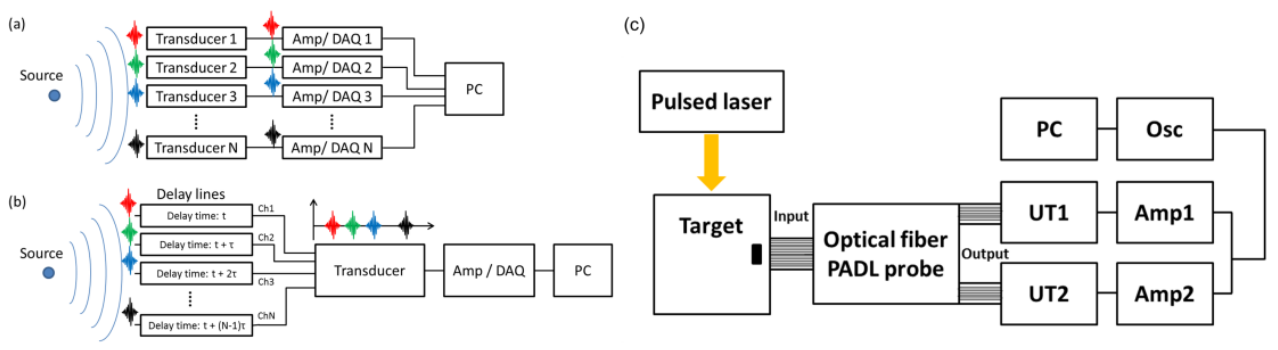

Figure 2.30 (a) PA Signal reception using conventional way (Multiple detectors and multi-channel DAQ), (b) PA signal reception using acoustic delay lines (Single element transducer and single channel DAQ) and (c) Experimental set up using 16-channel PADL probe. (Reprinted from ref. [49] Cho, Y. et al. 2014. Journal of Biomedical Optics, 19(8):086007. With permission from the authors and SPIE.)

The experimental setup is shown in Figure 2.30c. The light source used was a frequency doubled Q switched Nd:YAG laser (Continuum, SL2-10, San Jose, California) with a pulse duration of $5 \mathrm{~ns}$, repetition rate of $10 \mathrm{~Hz}$ and wavelength of $532 \mathrm{~nm}$. The PA waves generated were passed through the optical fiber PADL (parallel acoustic delay line) probe for adding 
required delay and acquired by two DAQ channels. In each DAQ channel, eight time delayed PA signals were sequentially detected by a single element unfocussed US transducer (Olympus NDT, V303). Detected signals were then averaged 10 times to generate images at a frame rate of $1 \mathrm{fps}$. The concept reported here was novel and handy for making the size of systems small and inexpensive. It could receive signals from 16 channels by just making use of 2 US detectors and two DAQ channels, which corresponded to a channel reduction ratio of 8:1. But the application of this method in-vivo is far from reality as lateral resolution depends on number of PADL's in use. It is practically difficult as of now to build complex probes with large number of PADLs with outsized length.

\subsubsection{The University of Washington system}

Jinjun Xia et al [14] recently reported on a system which they describe as being translational, and which is claimed to be a step for making US/PA dual mode imaging clinically acceptable. The expensive and bulky laser with low repetition rates $(10-20 \mathrm{~Hz})$ has been one of the main limitations of current research systems aiming at real-time PA imaging for patient use. This integrated US/PA system avoids these limitations by making use of a portable, low-cost, low pulse-energy fiber laser (HM 40W G3.1, SPI, South Hampton, UK), with repetition rates as high as $100 \mathrm{kHz}$. The laser system used is shown in Figure 2.31b. This is claimed to be potentially the next generation of light sources for real time PA imaging systems.

The low pulse energy around $1 \mathrm{~mJ} /$ pulse at $1064 \mathrm{~nm}$, requires a small spot of 1-2 mm diameter, which results in shallow penetration depths. To improve on this, the system uses fast spot scanning using a scanning galvo mirror (GVS001, Thorlabs, Newton, NJ, USA) to form a final synthesized PA image from single shot PA images. The system was built around the Verasonics (Verasonics, Redmond, WA, USA) US platform. The US transducer was a linear array probe with 64 elements (AT8L12-5 $50 \mathrm{~mm}$, Broadsound, Hsinchu, Taiwan), for both US and PA imaging. An FPGA module synchronized the US system and the galvo mirror based on the clock signal from US system. Figure 2.31a shows the control block diagram of the system. Over an imaging field of $2 \mathrm{~cm} \times 1 \mathrm{~cm}$, a combined frame rate of 32.8 $\mathrm{Hz}$ for both PA and US was achieved which is remarkable. The frame rate here could be improved by increasing the number of transducer elements and simultaneously acquiring data. 
(a)

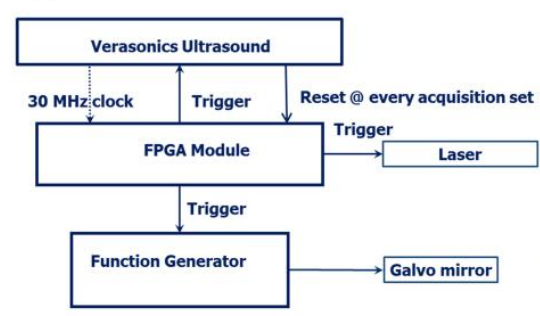

(b)

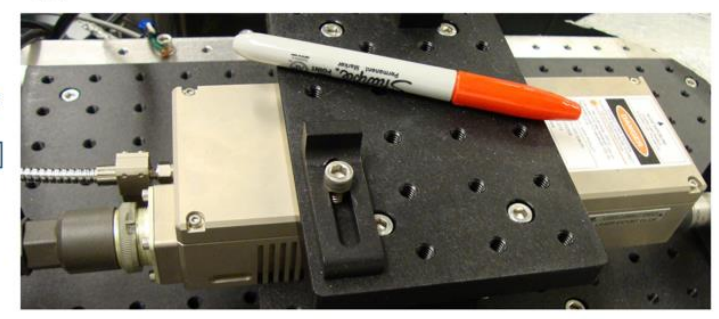

Figure 2.31 (a) Block diagram of integrated US/PA system, including a FPGA module and (b) Photograph of the used diode pumped laser system.(Adapted from ref. [14] Jinjun Xia et al. 2014. Proceedings. SPIE 8943,894310. With permission from the authors and SPIE.)

\subsubsection{The University of Texas system}

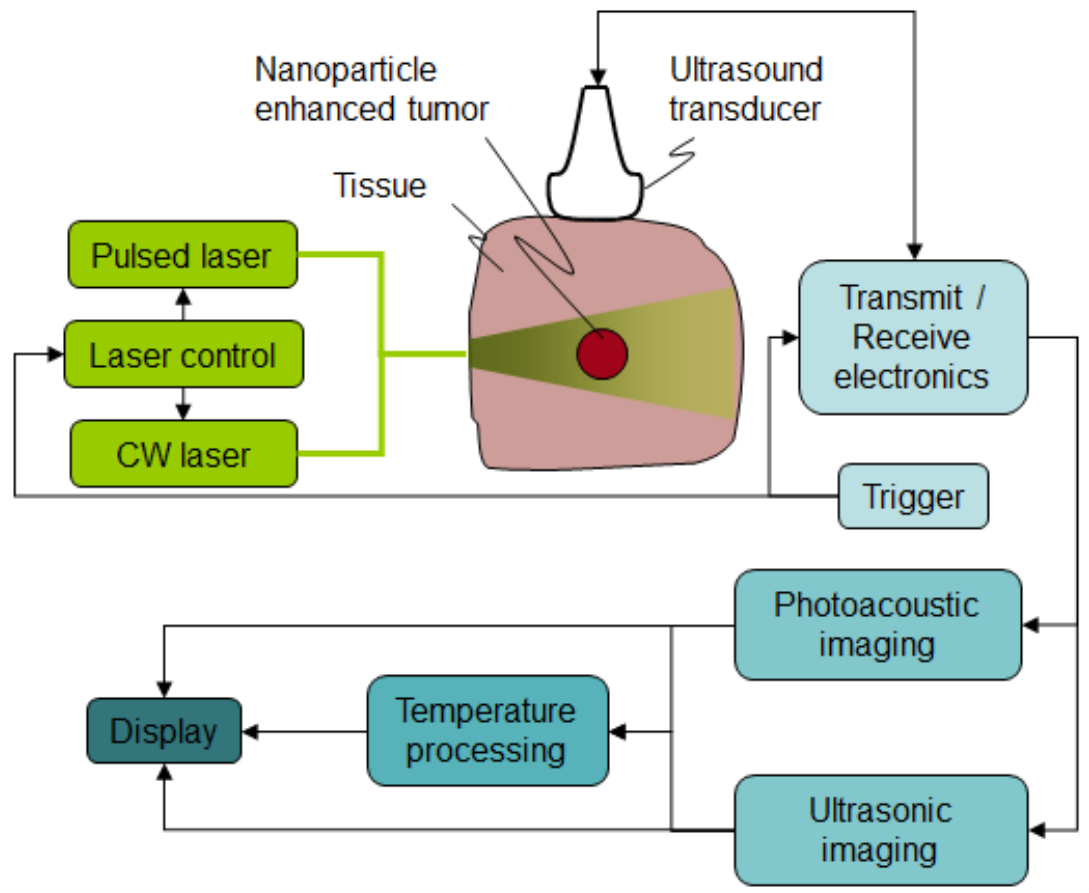

Figure 2.32 Experimental setup for PA and US imaging during therapy. (Reprinted from ref. [50] Shah, J. et al. 2008. Journal of Biomedical Optics, 13(3):034024. With permission from the authors and SPIE.) 
Shah et al [50] reported the feasibility of using PA imaging to detect photoabsorbers and to monitor temperature during photothermal therapy. To perform image-guided therapy, a laboratory prototype of a PA and US imaging system, consisting of a pulsed laser and an array-based US imaging system, was interfaced with a continuous wave therapeutic laser. This work is included in the review to showcase the promising application of image guided therapy using dual modality US/PA system. They integrated imaging and therapy system to acquire PA and US frames during photothermal therapy. Figure 2.32 shows the schematic of the experimental set-up.

A 128 element linear array transducer, with element spacing of about $300 \mu \mathrm{m}$, (SonixRP, Ultrasonix Medical Corporation, Canada) was used to capture US pulse-echo and PA data. The signals were logged using a 40-MHz sampling rate. The nominal center frequency of the transducer in the pulse-echo regime was 5MHz. An OPO pulsed laser system (Vibrant B, Opotek Incorporated, USA) operating at an 800-nm wavelength, with a 5-ns pulse duration and a $10-\mathrm{Hz}$ repetition rate providing optical fluence up to $15 \mathrm{~mJ} / \mathrm{cm}^{2}$ was interfaced for PA imaging. A continuous wave diode laser (HAM, Power Technology, Incorporated, USA), operating at $800 \mathrm{~nm}$ with a maximum power of $1 \mathrm{~W}$, was used as a light source for the photothermal therapy.

The authors performed photothermal therapy experiment on porcine tissue with injected photoabsorbers for proving the feasibility of using PA/US imaging for guidance and monitoring during therapy. US based thermal imaging utilized thermally induced speckle motion and PA based temperature measurement relied on temperature-induced amplitude variations in PA signal. PA and US techniques were compared for their sensitivity to monitor temperature changes during the therapy. PA based thermal imaging was found to be having higher CNR (Contrast to noise ratio) when compared to US thermal imaging. Figure 2.33a and $\mathrm{b}$ show the US and PA image of the region respectively. The site of the photoabsorber injection is indicated by the dashed circle in the US image, which also corresponds to the location of the strong PA response. For comparison, both US and PA imaging are used for thermal imaging. Figure $2.33 \mathrm{c}-\mathrm{f}$ show the PA based thermal images and Figure $2.33 \mathrm{~g}-\mathrm{j}$ show the US based thermal images. This Ex-vivo tissue study indicates that PA and US thermal imaging can be used together to compute temperature change during photothermal therapy with high sensitivity and good SNR throughout the imaging volume. Ke et al [51] recently reported that it is possible to measure the spatial distribution of temperature in deep 
tissue using PA imaging with a precision of $-0.8 \pm 0.4^{\circ} \mathrm{C}$ (mean \pm standard error). Since lot of clinically approved photoabsorbers for thermal therapy are available, future of PA/US based thermal imaging is bright.

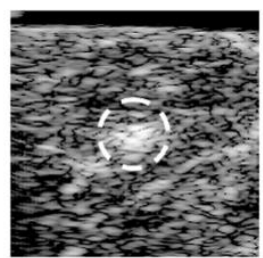

(a)

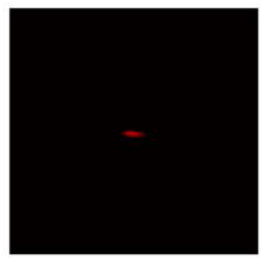

(c)

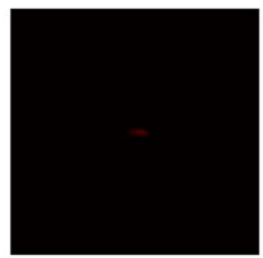

(g)

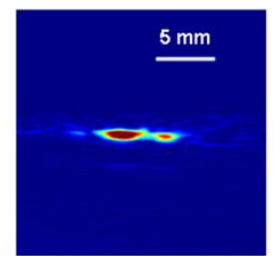

(b)

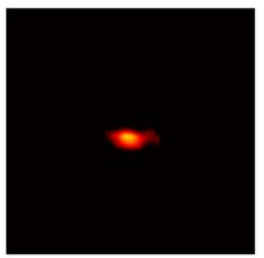

(d)

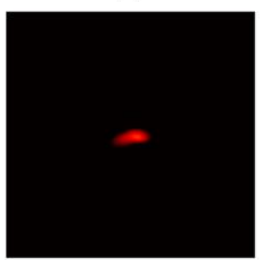

(h)

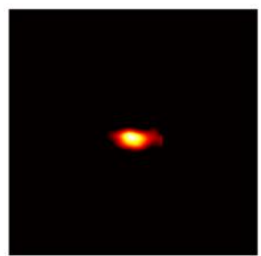

(e)

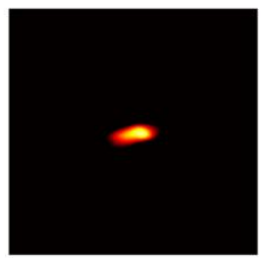

(i)
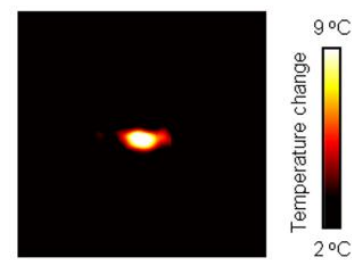

(f)

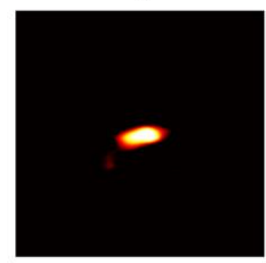

Figure 2.33 Photothermal therapy experiment on porcine tissue with injected photoabsorbers. (a) Initial US and (b) PA images. The injection site is marked by a dashed circle in the US image. (c) through (f) PA-based thermal images and (g) through (j) US-based thermal images after 60, 120, 180, and 240s of therapy. All images cover a $20 \times 20$-mm field of view. (Reprinted from ref. [50] Shah, J. et al. 2008. Journal of Biomedical Optics, 13(3):034024. With permission from the authors and SPIE.)

Table 2.1 summarizes the characteristics of all important handheld probe-based dual mode US/PA imaging systems reported in recent years. A few systems without US imaging capability are also included to show different technical advancements and applications. 


\begin{tabular}{|c|c|c|c|c|c|c|c|}
\hline $\begin{array}{c}\text { Research } \\
\text { group } \\
\text { (Published } \\
\text { year) } \\
\text { [References] }\end{array}$ & $\begin{array}{c}\text { No of } \\
\text { probe } \\
\text { elements }\end{array}$ & $\begin{array}{c}\text { Center } \\
\text { frequency } \\
\text { (MHz) }\end{array}$ & $\begin{array}{c}\text { Bandwidth } \\
\text { (-6 dB) } \\
(\%)\end{array}$ & $\begin{array}{c}\text { US } \\
\text { imaging } \\
\text { capability }\end{array}$ & $\begin{array}{c}\text { Laser } \\
\text { wavelength }\end{array}$ & $\begin{array}{c}\text { Laser } \\
\text { pulse } \\
\text { repetition } \\
\text { frequency } \\
(\text { PRF) } \\
(\text { Hz) }\end{array}$ & $\begin{array}{c}\text { Frame } \\
\text { rate } \\
(\mathbf{H z})\end{array}$ \\
\hline $\begin{array}{c}\text { Niederhauser } \\
\text { et al. (2005) } \\
{[27]}\end{array}$ & 64 & 7.5 & 76 & Yes & $760 \mathrm{~nm}$ & 7.5 & 7.5 \\
\hline $\begin{array}{c}\text { Kolkman et } \\
\text { al. (2008) } \\
{[35]}\end{array}$ & 128 & 7.5 & 75 & Yes & $1064 \mathrm{~nm}$ & 1000 & 8 \\
\hline $\begin{array}{l}\text { Kim et al. } \\
\text { (2010) [26] }\end{array}$ & 128 & 6 & 67 & Yes & Tunable dye & 10 & 1 \\
\hline $\begin{array}{l}\text { Haisch et al. } \\
\text { (2010) [37] }\end{array}$ & $96(192)$ & 8.8 & 50 & Yes & $\mathrm{OPO}$ & 100 & 1.1 \\
\hline $\begin{array}{c}\text { Fronheiser et } \\
\text { al. }(2010) \\
\text { [38] }\end{array}$ & 128 & 5 & 75 & No & $1064 \mathrm{~nm}$ & 10 & 10 \\
\hline $\begin{array}{c}\text { Montilla et } \\
\text { al. (2013) } \\
\text { [39] }\end{array}$ & 64 & $\approx 7.5$ & $\approx 67$ & Yes & $\mathrm{OPO}$ & 20 & 20 \\
\hline $\begin{array}{c}\text { Buehler et al. } \\
\text { (2013) [40] }\end{array}$ & 128 & 8 & 60 & No & OPO & 50 & 50 \\
\hline $\begin{array}{c}\text { Dean-Ben et } \\
\text { al. (2014) } \\
{[43]}\end{array}$ & 256 & 4 & 100 & No & $\mathrm{OPO}$ & 50 & $\approx 50$ \\
\hline $\begin{array}{l}\text { Needles et al. } \\
\text { (2013) [44] }\end{array}$ & 256 & 21 & 52 & Yes & OP0 & 20 & 5 \\
\hline $\begin{array}{l}\text { Yuan et al. } \\
\text { (2013) [46] }\end{array}$ & 128 & 11.25 & 75 & Yes & $\mathrm{OPO}$ & 10 & 10 \\
\hline $\begin{array}{l}\text { Xia et al. } \\
\text { (2014) [14] }\end{array}$ & 64 & $\approx 10$ & $\approx 70$ & Yes & $1064 \mathrm{~nm}$ & 1000 & 32 \\
\hline $\begin{array}{l}\text { Daoudi et al. } \\
\text { (2014) [36] }\end{array}$ & 128 & 7.5 & 100 & Yes & $800 \mathrm{~nm}$ & 1000 & 10 \\
\hline
\end{tabular}

Table 2.1 Comparison of different handheld probe based PA/US systems. 


\subsection{Concluding remarks, envisioned improvements and applications}

A PA imaging set-up consists of a laser illumination system attached to an US detector array and a data acquisition system for acquiring US signals. Commercially available US probes are not specifically designed to be sensitive for PA signal transduction. The relatively low amplitudes and wide frequency bands of PA signals call for custom-made sensitive and wide bandwidth transducers as receivers $[52,53]$ for PA imaging, which may not be appropriate for US imaging. In case of dual modality US/PA system, the selection of available commercial US probes is a compromise. Novel US probes with enhanced sensitivity and noise performance suitable for PA/US imaging are reported [54], but still in research stage. Commercial probes are centered around frequencies typically between 7.5 and $12 \mathrm{MHz}$, and possessing bandwidths under $80 \%$. This limits the sizes of objects that can be faithfully reconstructed to sub-mm sized structures.

Another key aspect of a PA/US system is the light delivery scheme. For deeper relevant penetration depths, high power Q-switched lasers are required which are typically expensive and bulky. These may not be suitable for a clinical setting. All the systems reviewed here excepting Ref. [14, 36] use bulky laser systems, even if the probes are handheld and compact. To maintain the portability of the US equipment, it is important to device handy and compact light sources that can provide reasonable energy for deep PA imaging. Another crucial element to be considered is the frame rate of the system, which is in turn dependent on the repetition rate of the laser. A Q-switched Nd:YAG laser commonly used for PA imaging have pulse repetition frequency (PRF) of ten to hundreds of hertz [55]. This limits the time resolution of the system. There are developments in this field and fast lasers capable of PRF up-to $100 \mathrm{kHz}$ have been reported recently [17]. Another aspect that is expected to improve in future is fast switching of wavelengths for PA spectroscopy. Novel techniques like delayed laser pulsing (per wavelength) are recently reported for avoiding movement artifacts during measurement using handheld probes [56]. Laser diode technology is also improving for providing pulsed light at higher rates and comparable energy [36]. However, repetition rate (thereby energy) is limited by the MPE. The frame rate of the system also depends upon how the system acquires and process (preprocess and reconstruct) the data for the final image. Developments in GPU (Graphical processing unit) and FPGA (Field Programmable Gate Array) technology are proving to be helpful in improving this, as parallel acquisition and 
processing is possible using these high-speed techniques. Compact, fast, and energy-efficient light sources together with sensitive and wide-band US signal detection, and high speed data acquisition and processing are the key factors for the translation of real-time PA/US imaging from research laboratories to clinics as a potential medical diagnostic technique.

Based on exciting early clinical investigation results, it is clear that PA/US imaging offers great promise in a wide range of clinical applications. The dual modality can provide powerful molecular and functional imaging capabilities in the future. Future applications in the clinic may be in imaging for multiple indications like cardiovascular and peripheral vascular disease, disorders related to lymphatic system, breast lesions, arthritis and inflammation. It is foreseen that a real-time and handheld multi-wavelength dual modality PA/US system will make an impact on clinical patient care in the future. 


\subsection{References}

1. M.I. Mishchenko, L.D. Travis, and A.A. Lacis, Scattering, Absorption, and Emission of Light by small Particles. 2002, Cambridge University Press: Cambridge.

2. R. Weissleder and M.J. Pittet, Imaging in the era of molecular oncology. Nature, 2008. 452(7187): p. 580-9.

3. R. Nachabe, B.H. Hendriks, M. Van Der Voort, A.E. Desjardins and H.J. Sterenborg, Estimation of biological chromophores using diffuse optical spectroscopy: Benefit of extending the uv-vis wavelength range to include 1000 to $1600 \mathrm{~nm}$. Biomedical Optics Express, 2010. 1(5): p. 1432-42.

4. P. Beard, Biomedical photoacoustic imaging. Interface Focus, 2011. 1(4): p. 60231.

5. V. Baeriswyl and G. Christofori, The angiogenic switch in carcinogenesis. Semin Cancer Biol, 2009. 19(5): p. 329-37.

6. P. Carmeliet and R.K. Jain, Angiogenesis in cancer and other diseases. Nature, 2000. 407(6801): p. 249-57.

7. N. Weidner, et al., Tumor angiogenesis and metastasis--correlation in invasive breast carcinoma. N Engl J Med, 1991. 324(1): p. 1-8.

8. L.V. Wang, Tutorial on photoacoustic microscopy and computed tomography. Ieee Journal of Selected Topics in Quantum Electronics, 2008. 14(1): p. 171-79.

9. S. Ricci, L. Moro, and R.A. Incalzi, Ultrasound Imaging of the Sural Nerve: Ultrasound Anatomy and Rationale for Investigation. European Journal of Vascular and Endovascular Surgery, 2010. 39(5): p. 636-641.

10. J. Shalhoub, et al., The use of Contrast Enhanced Ultrasound in Carotid Arterial Disease. European Journal of Vascular and Endovascular Surgery, 2010. 39(4): p. 381-387.

11. T. Szabo, Diagnostic Ultrasound Imaging: Inside Out. 2004, New York: NY:Elsevier Academic.

12. M. Tanter and M. Fink, Ultrafast imaging in biomedical ultrasound. IEEE Trans Ultrason Ferroelectr Freq Control, 2014. 61(1): p. 102-19.

13. D. Piras, W. Xia, W. Steenbergen, T.G. van Leeuwen, and S. Manohar, Photoacoustic Imaging of the Breast Using theTwente Photoacoustic Mammoscope: Present Status and Future Perspectives. Ieee Journal of Selected Topics in Quantum Electronics, 2010. 16(4).

14. J. Xia, C. Wei, T. Nguyen, B. Arnal, I. Pelivanov, and M. O'Donnell. Clinically Translatable Integrated Ultrasound and Photoacoustic Imaging System. in SPIE. 2014.

15. Y. Sun, H.B. Jiang, B.E. O'Neill, Photoacoustic Imaging: An Emerging Optical Modality in Diagnostic and Theranostic Medicine. Journal of Biosensors and Bioelectronics, 2011. 2(3).

16. F.A. Duck, Physical properties of tissue: a comprehensive reference book. 1990, London: Academic Press.

17. S. Manohar, et al., Initial results of in vivo non-invasive cancer imaging in the human breast using near-infrared photoacoustics. Opt Express, 2007. 15(19): p. 12277-85. 
18. A.G. Bell, On the production and reproduction of sound by light. American journal of Science, 1880. 20: p. 305-324.

19. R.A. Kruger, P. Liu, Y.R. Fang, and C.R. Appeldoorn, Photoacoustic ultrasound (PAUS) - reconstruction tomography. Med Phys, 1995. 22: p. 1605-1609.

20. A.A. Oraevsky, V.A. Andreev, A.A Karabutor, D. Fleming, Z. Gatalica, H. Singh, and R.O. Esenaliev, Laser optoacoustic imaging of the breast: detection of cancer angiogenesis. in SPIE. 1999.

21. P. Beard. Photoacoustic imaging of blood vessel equivalent phantoms. in SPIE. 2002.

22. B.T. Cox and P. Beard, Fast calculation of pulsed photoacoustic fields in fluids using k-space methods. Journal of the Acoustical Society of America, 2005. 117(6): p. 3616-3627.

23. B.T. Cox, J.G. Laufer, and P.C. Beard, The challenges for quantitative photoacoustic imaging. Photons Plus Ultrasound: Imaging and Sensing 2009, 2009. 7177.

24. P. Kuchment and L. Kunyansky, A survey in mathematics for industry mathematics of thermoacoustic tomography. European Journal of Applied Mathematics, 2008. 19: p. 191-224.

25. B.E. Treeby and B.T. Cox, $k$-Wave: MATLAB toolbox for the simulation and reconstruction of photoacoustic wave fields. J Biomed Opt, 2010. 15(2): p. 021314.

26. C. Kim, et al., Deeply penetrating in vivo photoacoustic imaging using a clinical ultrasound array system. Biomedical Optics Express, 2010. 1(1): p. 278-284.

27. J.J. Niederhauser, et al., Combined ultrasound and optoacoustic system for realtime high-contrast vascular imaging in vivo. Ieee Transactions on Medical Imaging, 2005. 24(4): p. 436-440.

28. C. Kim, et al., Performance benchmarks of an array-based hand-held photoacoustic probe adapted from a clinical ultrasound system for non-invasive sentinel lymph node imaging. Philos Trans A Math Phys Eng Sci, 2011. 369(1955): p. 4644-50.

29. R.J. Zemp, et al., Realtime photoacoustic microscopy in vivo with a $30-\mathrm{MHz}$ ultrasound array transducer. Optics Express, 2008. 16(11): p. 7915-7928.

30. R.G.M. Kolkman, et al., Real-time Photoacoustic \& Ultrasound imaging of human vasculature. Photons Plus Ultrasound: Imaging and Sensing 2009, 2009. 7177.

31. S. Park, et al., Integrated system for ultrasonic, photoacoustic and elasticity imaging - art. no. 61470H. Medical Imaging 2006: Ultrasonic Imaging and Signal Processing, 2006. 6147: p. H1470-H1470.

32. J. Jose, et al., Passive element enriched photoacoustic computed tomography (PER PACT) for simultaneous imaging of acoustic propagation properties and light absorption. Opt Express, 2011. 19(3): p. 2093-104.

33. X.D. Wang, et al., Photoacoustic Imaging with a Commercial Ultrasound System and a Custom Probe. Ultrasound in Medicine and Biology, 2011. 37(3): p. 484-492.

34. K.P. Kostli, et al., Optoacoustic imaging using a three-dimensional reconstruction algorithm. Ieee Journal of Selected Topics in Quantum Electronics, 2001. 7(6): p. 918-923.

35. R.G.M. Kolkman, et al., Real-time in vivo photoacoustic and ultrasound imaging. Journal of Biomedical Optics, 2008. 13(5). 
36. K. Daoudi, et al., Handheld probe integrating laser diode and ultrasound transducer array for ultrasound/photoacoustic dual modality imaging. Optics Express, 2014. 22(21): p. 26365-26374.

37. C. Haisch, et al., Combined optoacoustic/ultrasound system for tomographic absorption measurements: possibilities and limitations. Analytical and Bioanalytical Chemistry, 2010. 397(4): p. 1503-1510.

38. M.P. Fronheiser, et al., Real-time optoacoustic monitoring and three-dimensional mapping of a human arm vasculature. Journal of Biomedical Optics, 2010. 15(2).

39. L.G. Montilla, et al., Real-time photoacoustic and ultrasound imaging: a simple solution for clinical ultrasound systems with linear arrays. Physics in Medicine and Biology, 2013. 58(1): p. N1-N12.

40. A. Buehler, et al., Real-time handheld multispectral optoacoustic imaging. Optics Letters, 2013. 38(9): p. 1404-1406.

41. A. Dima and V. Ntziachristos, Non-invasive carotid imaging using optoacoustic tomography. Optics Express, 2012. 20(22): p. 25044-25057.

42. X.L. Dean-Ben and D. Razansky, Functional optoacoustic human angiography with handheld video rate three dimensional scanner. Photoacoustics, 2013. 1(2013): p. 68-73.

43. X.L. Dean-Ben and D. Razansky, Adding fifth dimension to optoacoustic imaging: volumetric time-resolved spectrally enriched tomography. Light-Science \& Applications, 2014. 3.

44. A. Needles, et al., Development and Initial Application of a Fully Integrated Photoacoustic Micro-Ultrasound System. Ieee Transactions on Ultrasonics Ferroelectrics and Frequency Control, 2013. 60(5): p. 888-897.

45. G.C. Langhout, et al., Detection of melanoma metastases in resected human lymph nodes by noninvasive multispectral photoacoustic imaging. Int J Biomed Imaging, 2014. 2014: p. 163652.

46. J. Yuan, et al., Real-time photoacoustic and ultrasound dual-modality imaging system facilitated with graphics processing unit and code parallel optimization. Journal of Biomedical Optics, 2013. 18(8).

47. G. $\mathrm{Xu}$, et al., Photoacoustic and ultrasound dual-modality imaging of human peripheral joints. Journal of Biomedical Optics, 2013. 18(1).

48. Y. Cho, et al., A Handheld Optical Fiber Parallel Acoustic Delay Line (PADL) Probe for Photoacoustic Tomography. Photons Plus Ultrasound: Imaging and Sensing 2014, 2014. 8943.

49. Y. Cho, et al., Handheld photoacoustic tomography probe built using optical-fiber parallel acoustic delay lines. Journal of Biomedical Optics, 2014. 19(8).

50. J. Shah, et al., Photoacoustic imaging and temperature measurement for photothermal cancer therapy. Journal of Biomedical Optics, 2008. 13(3).

51. H.X. Ke, S. Tai, and L.V. Wang, Photoacoustic thermography of tissue. Journal of Biomedical Optics, 2014. 19(2).

52. A.A. Karabutov, et al., Backward mode detection of laser-induced wide-band ultrasonic transients with optoacoustic transducer. Journal of Applied Physics, 2000. 87(4): p. 2003-2014.

53. W. Xia, et al., An optimized ultrasound detector for photoacoustic breast tomography. Med Phys, 2013. 40(3): p. 032901. 
54. S. Vaithilingam, et al., Three-Dimensional Photoacoustic Imaging Using a TwoDimensional CMUT Array. Ieee Transactions on Ultrasonics Ferroelectrics and Frequency Control, 2009. 56(11): p. 2411-2419.

55. R. Bouchard, O. Sahin, and S. Emelianov, Ultrasound-guided photoacoustic imaging: current state and future development. IEEE Trans Ultrason Ferroelectr Freq Control, 2014. 61(3): p. 450-66.

56. X.L. Dean-Ben, E. Bay, and D. Razansky, Functional optoacoustic imaging of moving objects using microsecond-delay acquisition of multispectral threedimensional tomographic data. Scientific Reports, 2014. 4. 


\section{Photoacoustic-guided}

\section{focused ultrasound (PAFUSion)}

\section{for identifying reflection artifacts}

\section{in photoacoustic imaging ${ }^{2}$}

Influence of acoustic inhomogeneities and resulting reflection artifacts is an important problem in reflection-mode photoacoustic imaging. Absorption of light by skin and superficial optical absorbers will generate photoacoustic transients, which traverse into the tissue and get reflected from structures having different acoustic impedance. These reflected photoacoustic signals, when reconstructed, may appear in the region of interest, which causes difficulties in image interpretation. We propose a novel method to identify and potentially eliminate reflection artifacts in photoacoustic images using photoacoustic-guided focused ultrasound [PAFUSion]. Our method uses focused ultrasound pulses to mimic the wave field produced by photoacoustic sources and thus provides a way to identify reflection artifacts in clinical combined photoacoustic and pulse-echo ultrasound. Simulation and phantom results are presented to demonstrate the validity and impact of this method. Results show that PAFUSion can identify reflections in photoacoustic images and thus envisages potential for improving photoacoustic imaging of acoustically inhomogeneous tissue.

\footnotetext{
2 This chapter has been published as: M. Kuniyil Ajith Singh, and W. Steenbergen; "Photoacoustic-guided focused ultrasound (PAFUSion) for identifying reflection artifacts in photoacoustic imaging," Photoacoustics 3(4), 123-131 (2015).
} 


\subsection{Introduction}

Photoacoustic (PA) imaging is a promising biomedical imaging modality that has emerged over the last decade. In PA imaging, pulsed light absorbed by the target emits thermoelastically generated ultrasound (US). This data can be detected using US probes allowing the reconstruction of location and spatial details of the light-absorbing target $[1,2]$. PA imaging thus combines the advantages of US and optical imaging, providing excellent optical contrast with ultrasonic resolution. While US imaging makes use of acoustic scattering and reflection in tissue to provide structural details, PA imaging extracts functional information based on optical absorption by tissue chromophores such as blood. Since PA imaging involves US detection, it can be realized in a commercially available US scanner to perform dual mode PA/US imaging [3-5]. These dual mode systems preferably utilize a scheme in which tissue is irradiated from the same side where PA signals are detected (reflection-mode, epi-mode PA imaging) [6]. This mode in which optical components and US transducers are combined, aids the clinician to perform single hand guidance of the probe during imaging. In addition, epi-illumination mode facilitates the imaging of body parts where bones or acoustically attenuating soft tissue would obstruct propagation of acoustic waves from the illuminated tissue region to the acoustic probe.

In many of the reported handheld probe-based PA/US systems, light illumination for PA imaging is done at an oblique angle in such a way that it coincides with the US imaging plane [3-5] with the goal to maximize fluence and thus signal-to-noise ratio. On the downside this results in a high light fluence on the surface of the tissue just beneath the US probe, such that melanin and superficial blood vessels generate strong PA transients which propagate into the tissue and reflect back from acoustically dense structures [7-9]. These reflected signals appear as artifacts in the reconstructed PA images. The reflection artifacts significantly reduce the contrast, and thus the imaging depth, which is critical [7, 8]. Since these artifacts are triggered by the properties of the tissue, simple signal averaging is not effective for reducing them. Because of the strong optical attenuation in tissue [1], reflection artifacts that show up in a certain depth can become stronger than the PA signals of interest in spite of the low level of acoustic scattering [10], which can limit the imaging depth. As an example, when imaging structures like finger joints (multiple light absorbers and acoustic reflectors), signals of interest may get mixed with the reflections from bone or tendon, which result in the wrong 
interpretation of images. To achieve clinically relevant PA imaging depth, it is vital to develop methods for eliminating reflection artifacts. Another key reason for limited PA imaging depth is the clutter caused by PA sources outside the imaging plane. These sources may produce high PA transients that could reach the imaging probe directly (direct clutter) or could get reflected on structures inside the imaging plane (echo clutter). PAFUSion deals with identifying reflections caused by PA sources within the imaging plane.

Different techniques have been reported to reduce clutter in PA imaging [7, 8, 10-13]. Deformation Compensated Averaging (DCA) [7] employs the artifact decorrelation, which is the result of tissue deformation when tissue is slightly palpated with the imaging probe. Although this technique showed promising results, notable disadvantages have been reported: 1) controlled probe motion can be performed only by an experienced person and this technique can be employed only for easily deformable tissue, and 2) the maximum achievable tissue deformation on one side limited by the tissue mechanical properties, and the minimum deformation required for artifact decorrelation on the other side, determines the contrast improvement. Another technique employs localized vibration tagging [LOVIT] of tissue using acoustic radiation force $[\mathrm{ARF}]$ for reducing clutter in the focal region of a longpulsed ultrasonic beam [6]. While claiming nearly full artifact elimination, authors also reported the difficulty in eliminating echo clutter completely using LOVIT. For successful echo clutter reduction, LOVIT prefers a small ARF displacement region, which sets a limitation to the real-time capability because extensive scanning is then required to achieve clutter reduction in a large field-of-view. This method also requires transducers that are capable of transmitting ARF pulses and is limited by the US safety regulations.

In this paper, we investigate a novel method that can identify and potentially eliminate reflection artifacts in PA images. We specifically aim at reducing the reflection artifacts caused by PA sources within the imaging plane. Our technique ultrasonically simulates the PA signal from an optical absorber and uncovers PA signal reflections caused by acoustic reflectors beneath or around the absorber. The described method does not require any additional transducers or computationally intense algorithm to identify reflection artifacts, thus foresees good potential in improving real-time clinical PA imaging. Compared to LOVIT, our method works with low US power and thus does not pose any risks in clinical application. Because the focused pulse-echo acquisitions in PAFUSion can be performed at a much higher frame rate than PA imaging, it holds potential to be fast and real time capable. 
Compared to DCA, it is not limited by tissue deformability and can thus even be used when e.g. imaging close to bone as in finger joint imaging. PA sources located outside the imaging plane are not directly observable and thus are not amenable to PAFUSion. Therefore, PAFUSion specifically targets at identifying and potentially reducing the reflection artifacts in a situation where tissue is irradiated as close as possible to the imaging plane with the goal to optimize signal-to-noise ratio, where out-of-plane clutter plays an inferior role.

The main goal of this paper is to present the first simulations and experimental results as a proof-of-principle of 'PAFUSion'.

\subsection{Theory}

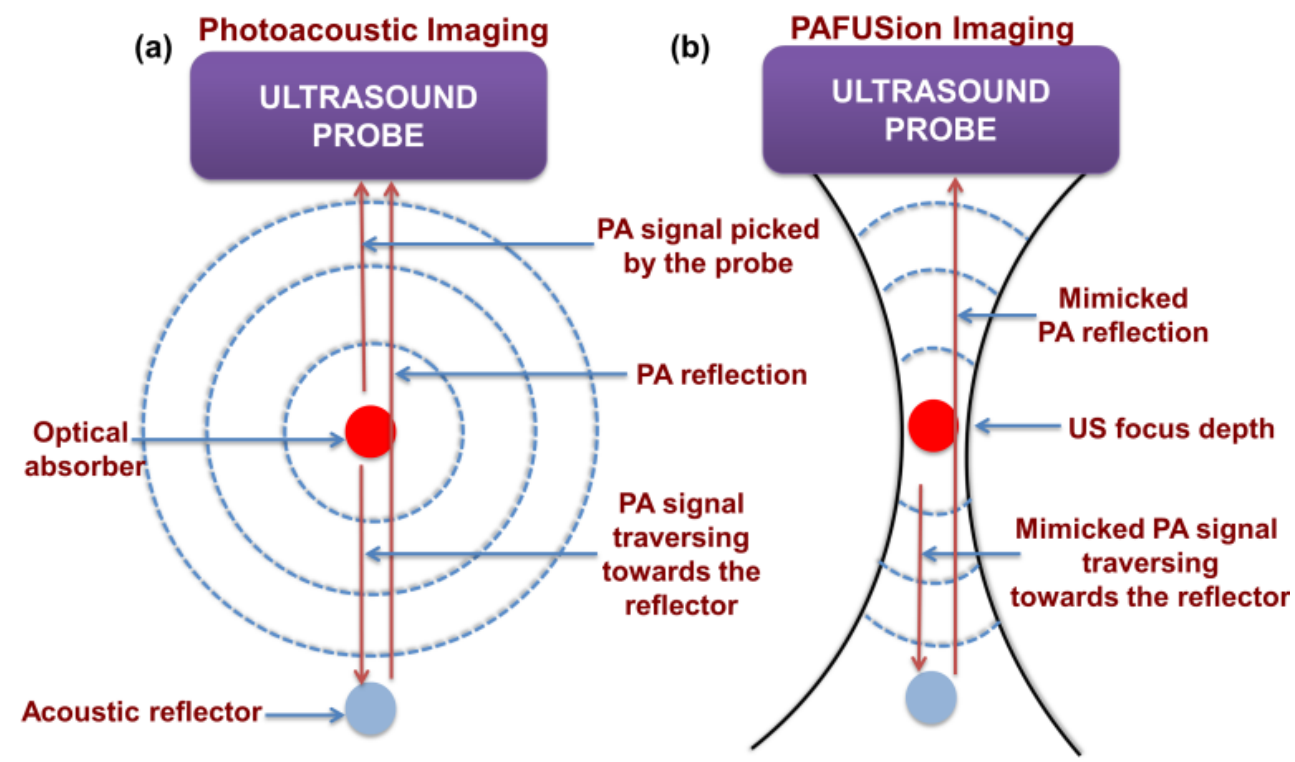

Figure 3.1 (a) Illustration of PA signal generation and signal causing reflection artifacts,

(b) Principle of PAFUSion: Focusing US to the PA source mimics the reflection caused by it.

Figure 3.1a illustrates the generation of a PA signal from an optical absorber, and the resulting PA reflection signal caused by an acoustic reflector deeper inside the tissue. In PAFUSion (Figure 3.1b), we transmit a focused US pulse, with the focal position adjusted to the location of the optical absorber by using guidance of PA data. The focused US pulse, at 
the time of arrival in the focal position, mimics the part of the PA wave that was traversing towards the reflector. Under the assumption that signal acquisition starts at the time when the focused US pulse arrives at the PA source $(t=0)$, the resulting signal will show the US reflections at the same time where they show up in the PA signal. When reconstructing a PA image from the US data, it will thus mimic the reflection artifacts in terms of shape and depth without containing the real PA signal itself.

Thus, instead of identifying reflectors, which would be done with normal US imaging, with PAFUSion we identify PA signal reflections, which then can be used to correct PA images for reflection artifacts.

\subsection{Methods and materials}

\subsubsection{PAFUSion - Processing steps}

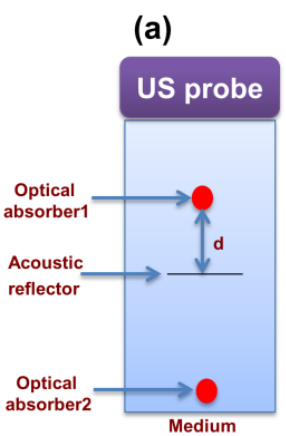

(b)

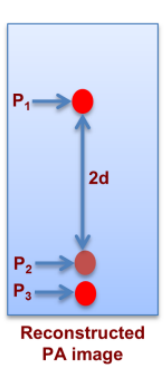

(c)
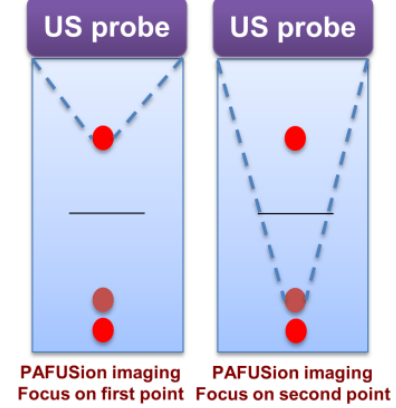

(e)

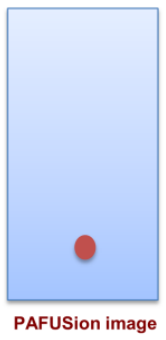

(f)

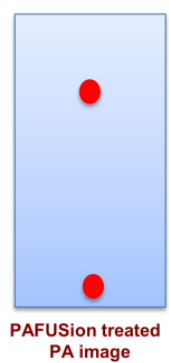

Figure 3.2 Step-by- step schematic illustration of data processing steps in PAFUSion.

(a) Simple medium with two optical absorbers and one acoustic reflector, (b) PA image,

(c) PAFUSion imaging step 1 - US focus on first high-intensity point in the PA image,

(d) PAFUSion imaging step 2 - US focus on second high-intensity point in the PA image, (e) PAFUSion image obtained using weighted addition of images obtained in (c) and (d), (f) PA image corrected using PAFUSion image.

The processing steps of PAFUSion are schematically illustrated in Figure 3.2. Let us consider a simple medium with two optical absorbers and one acoustic reflector in between them. The distance between optical absorber 1 and the acoustic reflector is $d$ as shown in Figure 3.2a. Once after collecting PA data, an image can be reconstructed using any reconstruction 
algorithm. Figure 3.2b shows the schematic of the reconstructed PA image, in which three high intensity points $\mathrm{P}_{1}, \mathrm{P}_{2}$, and $\mathrm{P}_{3}$ are visible. Points $\mathrm{P}_{1}, \mathrm{P}_{2}$, and $\mathrm{P}_{3}$ have intensities $\mathrm{I}_{1}, \mathrm{I}_{2}$, and $\mathrm{I}_{3}$ respectively. Intensity for a particular point is defined here as the maximum value of the reconstructed image envelope at the location of that point. The distance between $\mathrm{P}_{1}$ and $\mathrm{P}_{2}$ is $2 \mathrm{~d}$. This PA image is used as the guidance for further steps in the technique. PAFUSion is applied on points from top to bottom in PA image.

In the example in Figure 3.2, three high intensity points are identified in the PA image, thus two steps are required to perform PAFUSion. It is not necessary to apply PAFUSion on $\mathrm{P}_{3}$, as this deepest feature in the PA image will never lead to the identification of again a new reflection. In the first step, US is focused onto $\mathrm{P}_{1}$ (Figure $3.2 \mathrm{c}$ ) and the resulting echoes are acquired. Reconstruction from this echo data is performed considering one-way propagation of sound and by setting the acquisition start $(\mathrm{t}=0)$ to the time at which US reaches the focus distance. By this way, reconstruction treats the spatial pressure distribution of the focused US pulse as the initial pressure distribution of a virtual PA source, and thus will be able to mimic the reflection artifacts caused by that PA source. The image obtained by this step is saved for further processing. In the second step, the same procedure is repeated for $\mathrm{P}_{2}$ (Figure 3.2d). Since there is no acoustic reflector beneath $\mathrm{P}_{2}$ in this example, resultant image of the second step will be blank without any echoes. During each step, US reflections from the depth of focus as well as above it are omitted from the reconstructed image. The next step is weighted addition of images obtained in step 1 and step 2 for obtaining the PAFUSion image (Figure 3.2e), which reveals only the reflection artifacts. The weights for the images obtained in steps 1 and 2 are chosen to be proportional to the intensities $I_{1}$ and $I_{2}$ respectively in the PA image in Figure 3.2a. As a final step, we envisage that the PAFUSion image can be used to correct the PA image for obtaining a reflection artifact-free PA image (Figure 3.2f). However, this proof-of-principle study focuses only on identifying the reflection artifacts in PA images. Correcting the PA images using PAFUSion images will be realized as a next stage of improvement.

\subsubsection{Equipment and setup}

A handheld dual mode PA/US system which was already reported by our group [5] was used for all the experiments in this study. Figure 3.3 shows the photograph of the system in which a commercial US scanner (MyLabOne, Esaote Europe BV, The Netherlands) was used along 
with a probe that integrates an US array with a diode laser module emitting pulses at $805 \mathrm{~nm}$ wavelength, $130 \mathrm{~ns}$ pulse width and pulse energy of $0.56 \mathrm{~mJ}$. The US probe has a $-6 \mathrm{~dB}$ fractional bandwidth of around $100 \%$ and center frequency of $7.5 \mathrm{MHz}$.

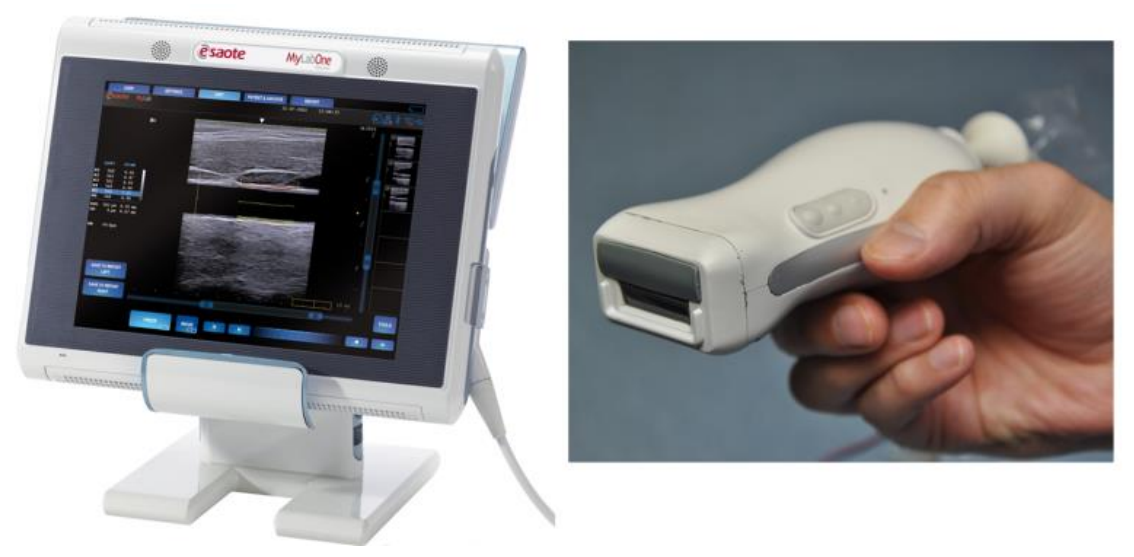

Figure 3.3 Portable US scanner (left) and the hybrid probe (right) integrating laser module and US transducer array [5].

The system was used in research mode where US transmission, laser pulse transmission, and data acquisition were controlled using custom-made software running on a PC. The same software also controlled the switching between PA imaging and US focusing for PAFUSion technique. In addition, plane-wave US images were acquired as a reference to monitor reflector positions. US focusing was achieved by adjusting the transmission delays of the different transducer elements in the linear array. By this way, it is possible to scan the focus to any point in the imaging plane. A one-cycle transmission pulse shape was applied to each transducer elements. In plane-wave US, PA, and PAFUSion imaging, RF data of all US transducer elements were saved after being acquired by the scanner with $50 \mathrm{MHz}$ sampling frequency and digitized with a dynamic range of 12 bits. PA images and reference plane wave US images, were reconstructed using a frequency domain reconstruction algorithm [14]. PAFUSion data was processed using the PA reconstruction algorithm by following the steps mentioned in section 3.3.1. 


\subsubsection{PAFUSion k-Wave simulation}

A 3-D simulation of the PAFUSion algorithm was performed using the k-Wave toolbox [15]. Figure 3.4a shows the geometry of the digital phantom and the acoustic parameters that were defined for the simulation. Simulation involved three steps: 1) PA imaging and reconstruction 2) focused US imaging 1 (focus to first high intensity point in the PA image), and 3) focused US imaging 2 (focus to second high intensity point in the PA image). Finally weighted addition of images (weight proportional to the PA intensity at the depths of focus) obtained in step 2 and 3 was performed for obtaining the PAFUSion image. For all the steps, reconstruction was done using a 2-D frequency domain reconstruction algorithm [14].

\subsubsection{Phantom measurements}

Three phantom measurements were performed for proving the validity of the PAFUSion technique to identify reflection artifacts in PA imaging. Before all experiments, measurements were done by moving the US/PA probe forth and back axially to identify potential reflection artifacts caused by optical absorption on the transducer surface that would move relative to the PA signal. In that way we made sure that all the reflection artifacts are really caused by PA sources inside the phantom. The first phantom was similar to the digital phantom in the simulation study. Figure 3.5a shows the schematic of this phantom in which one optical absorber (nylon thread) and acoustic reflector (delrin rod) was used. Both the optical absorber and acoustic reflector were positioned inside a tank filled with water in such a way that they were perpendicular to the imaging plane of the US/PA probe that was immersed in water (Figure 3.5a). This phantom was used to study a simple situation with a single PA reflection and its identification using the PAFUSion technique. Water without any scattering was chosen as the medium in this phantom for obtaining maximum PA signal (and thereby PA reflection) from the absorber positioned at the top part of the phantom.

The second phantom (Figure 3.6a) contained two optical absorbers and one acoustic reflector. Again, nylon threads and delrin rod were used as optical absorbers and acoustic reflector respectively. The second nylon thread was placed deep inside the phantom in such a way that a PA reflection artifact (caused by first nylon thread PA signal reflecting on delrin rod) would almost overlap with the PA signal from this second thread. This phantom was intended to study the fusion of PA reflections with signals of interest. This is a critical 
problem in imaging structures like finger joints, where multiple absorbers and acoustic reflectors are present. Water mixed with Intralipid $\left(\mu_{\mathrm{s}}{ }^{\prime}=6 \mathrm{~cm}^{-1}\right)$ was used as the medium in this phantom to make sure that both nylon threads generated a significant PA signal.

The nylon thread that was used as the optical absorber was itself acoustically reflective, thus it can cause reflection artifacts even in the absence of additional acoustic reflectors. The third phantom (Figure 3.7a) represents the condition in which optical absorbers themselves are acoustically reflective, and was meant to study and identify the resulting reflection artifacts. Two nylon threads were kept at different depths in water and were perpendicular to the US/PA probe (Figure 3.7a). Water without any scattering was chosen as the medium in this phantom for obtaining a strong PA signal from the first nylon thread (and thereby a strong reflection interfering with the second nylon thread) by directing the light to the top part of the phantom.

\subsection{Results}

In all the results, lateral and axial coordinates are represented by $\mathrm{x}$ and $\mathrm{z}$ respectively and the envelope of the images (PA, PAFUSion and US) are plotted in linear amplitude scale. Image reconstruction assumed constant speed of sound in the medium for all phantom measurements, which may have resulted in a slight deviation of the depth of reconstructed features compared to the physical depth owing to the inhomogeneous speed of sound of the embedded inclusions.

\subsubsection{PAFUSion simulation}

Figure 3.4a shows the details about the phantom and US probe positioning used for the PAFUSion simulation. Figure $3.4 \mathrm{~b}$ shows the reconstructed PA image. The PA source $(\mathrm{x}=$ $8 \mathrm{~mm}, \mathrm{z}=4.5 \mathrm{~mm})$ and its reflection on the acoustic reflector which occurs at position $(\mathrm{x}=$ $8 \mathrm{~mm}, \mathrm{z}=9.5 \mathrm{~mm}$ ) are evident in the PA image. Limited view artifacts in the shape of circular streaks are visible in both PA image and PAFUSion images. The PAFUSion image (Figure 3.4c) clearly identifies the PA reflection artifact by reproducing the correct depth and approximately the correct shape. It is evident from the results that PAFUSion could reproduce even the minute layered artifacts seen below the high intensity reflection $(x=8 \mathrm{~mm}, \mathrm{z}=9.5$ $\mathrm{mm})$. The intensity of the artifacts is different in the PAFUSion image than in the PA image 
because the simulated US transmission pressure amplitude was chosen independent from the PA signal amplitude.

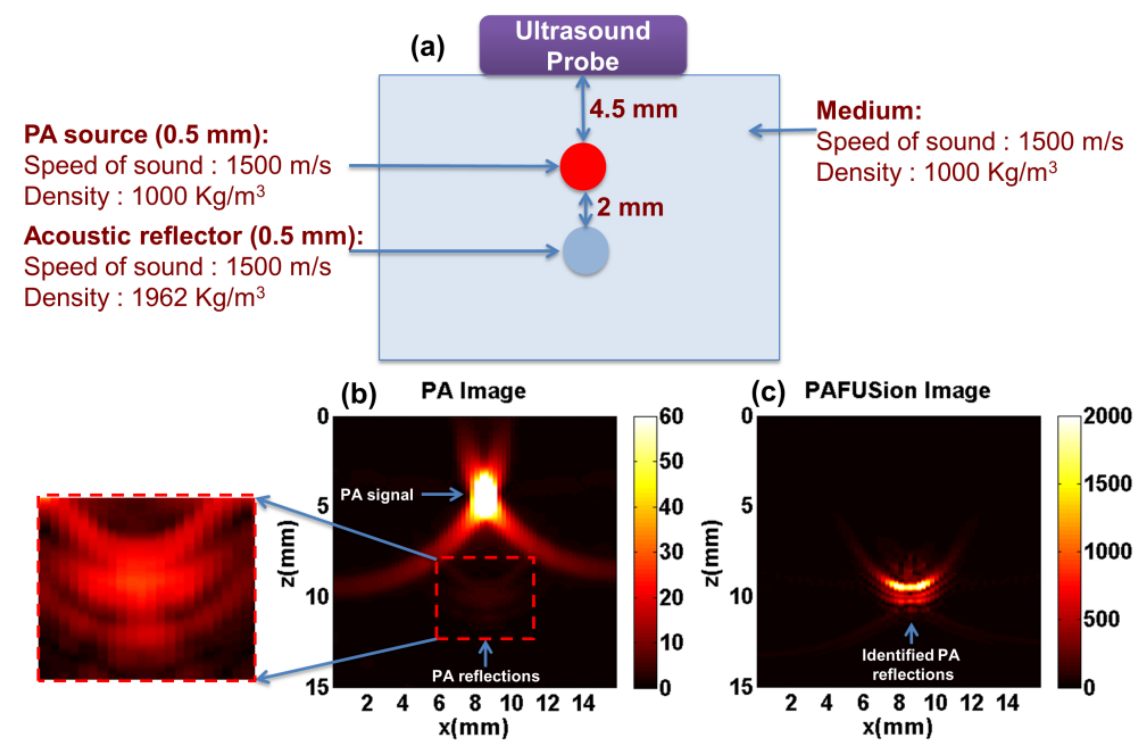

Figure 3.4 (a) Digital phantom used for simulation, including the acoustic properties, (b) PA image and enlarged region of interest, which shows the reverberation-like details of the reflection artifacts, (c) PAFUSion image.

\subsubsection{Phantom experiments}

Figure 3.5a shows the details about the first phantom and the schematic of the measurement setup. This phantom had only one optical absorber embedded and an artifact free PA image would have only one high intensity spot. Figure $3.5 \mathrm{~b}$ shows the reconstructed PA image in which the signal from the nylon thread $(\mathrm{x}=8 \mathrm{~mm}, \mathrm{z}=4.5 \mathrm{~mm})$ and two other signals $(\mathrm{x}=8$ $\mathrm{mm}, \mathrm{z}=7 \mathrm{~mm}$ and $\mathrm{x}=8 \mathrm{~mm}, \mathrm{z}=9.5 \mathrm{~mm})$ are visible. The nylon thread itself $(\mathrm{x}=8 \mathrm{~mm}, \mathrm{z}$ $=4.5 \mathrm{~mm})$ and two surfaces of delrin $\operatorname{rod}(\mathrm{x}=8 \mathrm{~mm}, \mathrm{z}=5.9 \mathrm{~mm}$ and $\mathrm{x}=8 \mathrm{~mm}, \mathrm{z}=7.3 \mathrm{~mm})$ are observable in the plane wave US image (Figure 3.5c). The PAFUSion image (Figure $3.5 \mathrm{~d}$ ) evidently revealed two reflection signals and confirmed that signals at $\mathrm{x}=8 \mathrm{~mm}, \mathrm{z}=7$ $\mathrm{mm}$ and $\mathrm{x}=8 \mathrm{~mm}, \mathrm{z}=9.5 \mathrm{~mm}$ in the PA image are reflection artifacts. It is worth mentioning that reflection artifacts identified by PAFUSion reproduced the shape and spatial details of the actual PA reflection artifacts quite well. The second reflection artifact $(\mathrm{x}=8 \mathrm{~mm}, \mathrm{z}=9.5$ 
$\mathrm{mm}$ ) is caused by the echo of the nylon thread PA signal on the second surface of the delrin rod. An extended horizontal feature can be seen right to the first identified reflection artifact $(\mathrm{x}=8 \mathrm{~mm}, \mathrm{z}=7 \mathrm{~mm}$ ) in the PAFUSion image (Figure 3.5d). This is potentially a reconstruction artifact common to the frequency-domain algorithm, which is better visible in the PAFUSion image than in the PA image owing to a higher center frequency of the transmitted US compared to the PA signal. These artifacts occur outside the region of interest and are thus not critical for this experiment. The intensity ratio of the two identified reflections $(\mathrm{x}=8 \mathrm{~mm}, \mathrm{z}=7 \mathrm{~mm}$ and $\mathrm{x}=8, \mathrm{z}=9.5 \mathrm{~mm}$ ) in the PAFUSion image ideally would be the same as the ratio of reflection intensities in the PA image. However, this ratio was found to be different in the PAFUSion and PA experiments (10 times higher in PAFUSion images). The intensities of the second reflections appear similar in the PA image and the PAFUSion image, because the color map in the PAFUSion image was chosen such that the pixel intensity of the first artifact was saturated to portray both artifacts clearly.

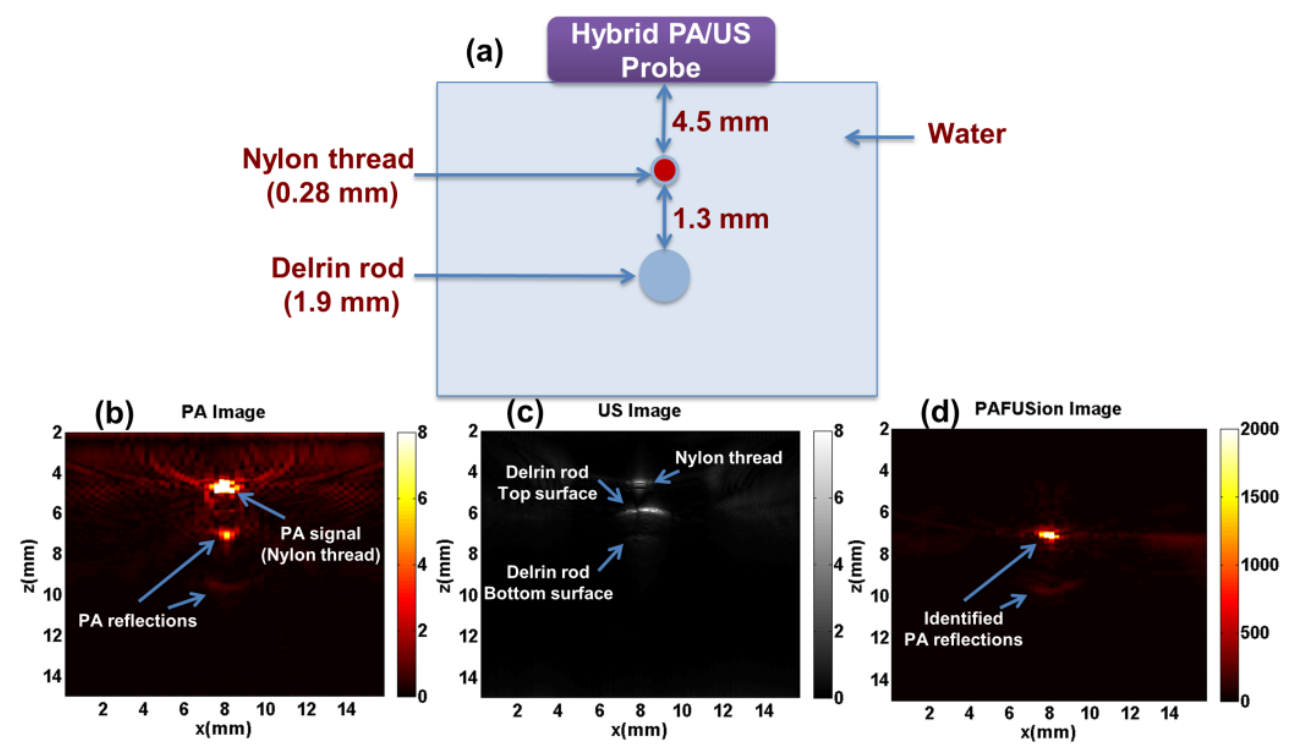

Figure 3.5 (a) Scheme of phantom 1 and experimental setup, (b) PA image, (c) plane wave US image, (d) PAFUSion image.

Figure 3.6a shows the details about the second phantom and the schematic of the arrangement used for the measurement. This phantom consisted of two optical absorbers and an acoustic reflector. Ideally, one would expect only two high intensity spots in the PA image. 
Figure 3.6b shows the reconstructed PA image in which the signals from the two nylon threads $(\mathrm{x}=8 \mathrm{~mm}, \mathrm{z}=5 \mathrm{~mm}$ and $\mathrm{x}=9.065 \mathrm{~mm}, \mathrm{z}=10.4 \mathrm{~mm})$ and two other signals $(\mathrm{x}=8$, $\mathrm{z}=7.4$ and $\mathrm{x}=8, \mathrm{z}=9.8)$ are visible. Both nylon threads $(\mathrm{x}=8 \mathrm{~mm}, \mathrm{z}=5 \mathrm{~mm}$ and $\mathrm{x}=9.065$, $\mathrm{z}=10.4 \mathrm{~mm})$ and two surfaces of delrin $\operatorname{rod}(\mathrm{x}=8 \mathrm{~mm}, \mathrm{z}=6.3 \mathrm{~mm}$ and $\mathrm{x}=8, \mathrm{z}=7.7 \mathrm{~mm})$ are noticeable in the plane wave US image (Figure 3.6c). The PAFUSion image (Figure 3.6d) clearly revealed two reflection signals and confirmed that signals at $x=8 \mathrm{~mm}, \mathrm{z}=7.4 \mathrm{~mm}$ and $\mathrm{x}=8 \mathrm{~mm}, \mathrm{z}=9.8 \mathrm{~mm}$ in PA image are reflection artifacts. The second reflection artifact $(\mathrm{x}=8 \mathrm{~mm}, \mathrm{z}=9.8 \mathrm{~mm})$ is almost fused with the signal from the second nylon thread $(\mathrm{x}=$ $9.065 \mathrm{~mm}, \mathrm{z}=10.4 \mathrm{~mm}$ ), mimicking a clinical scenario where identification of such an artifact would be crucial. The extended horizontal feature right to the first reflection in the PAFUSion image is again visible. This feature occurs outside the region of interest and thus does not prevent the identification of reflection artifacts. The distance between the first nylon thread and the delrin rod in this phantom is the same as for the first phantom. Also the intensity ratios of the two identified reflections $(x=8 \mathrm{~mm}, \mathrm{z}=7.4 \mathrm{~mm}$ and $\mathrm{x}=8 \mathrm{~mm}, \mathrm{z}=9.8$ $\mathrm{mm}$ ) were similar to the ones found in phantom 1 . This is reasonable because the distance travelled by the US (PAFUSion, PA imaging) and thus the attenuation was similar in phantom 1 and phantom 2.

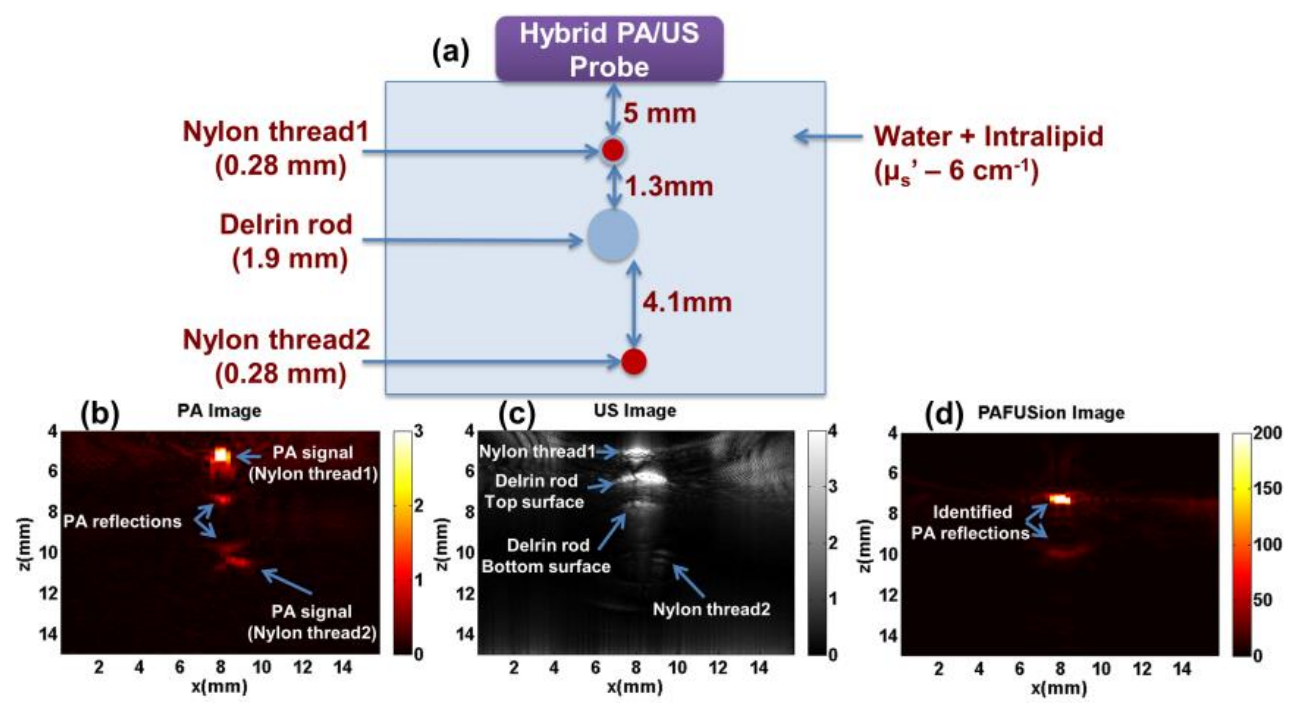

Figure 3.6 (a) Scheme of phantom 2 and experimental setup, (b) PA image, (c) plane wave US image, (d) PAFUSion image. 
The third phantom was designed to simulate the condition of optical absorbers that themselves reflect PA signals from other PA sources. Recent studies shown that in PA finger imaging, the tendon shows contrast in PA as well as US imaging [16]. If the skin/blood vessel signal gets reflected on the tendon, the resulting artifacts can make image interpretation difficult.

Figure 3.7a shows the details of the third phantom and the schematic of the measurement setup. Figure 3.7b shows the reconstructed PA image (zoomed in for more details) in which signals from the two nylon threads $(x=8 \mathrm{~mm}, \mathrm{z}=4 \mathrm{~mm}$ and $\mathrm{x}=8 \mathrm{~mm}, \mathrm{z}=6.2 \mathrm{~mm})$ and another signal $(\mathrm{x}=8 \mathrm{~mm}, \mathrm{z}=8.5 \mathrm{~mm})$ are evident. Both nylon threads are visible in the plane wave US image (Figure 3.7c). The PAFUSion image (Figure 3.7d) clearly exposes the reflection signal and confirms that the feature at $\mathrm{x}=8 \mathrm{~mm}, \mathrm{z}=8.5 \mathrm{~mm}$ in the PA image is a reflection artifact. It is worth mentioning that the PAFUSion image reproduces the depth and shape of the reflection artifact.

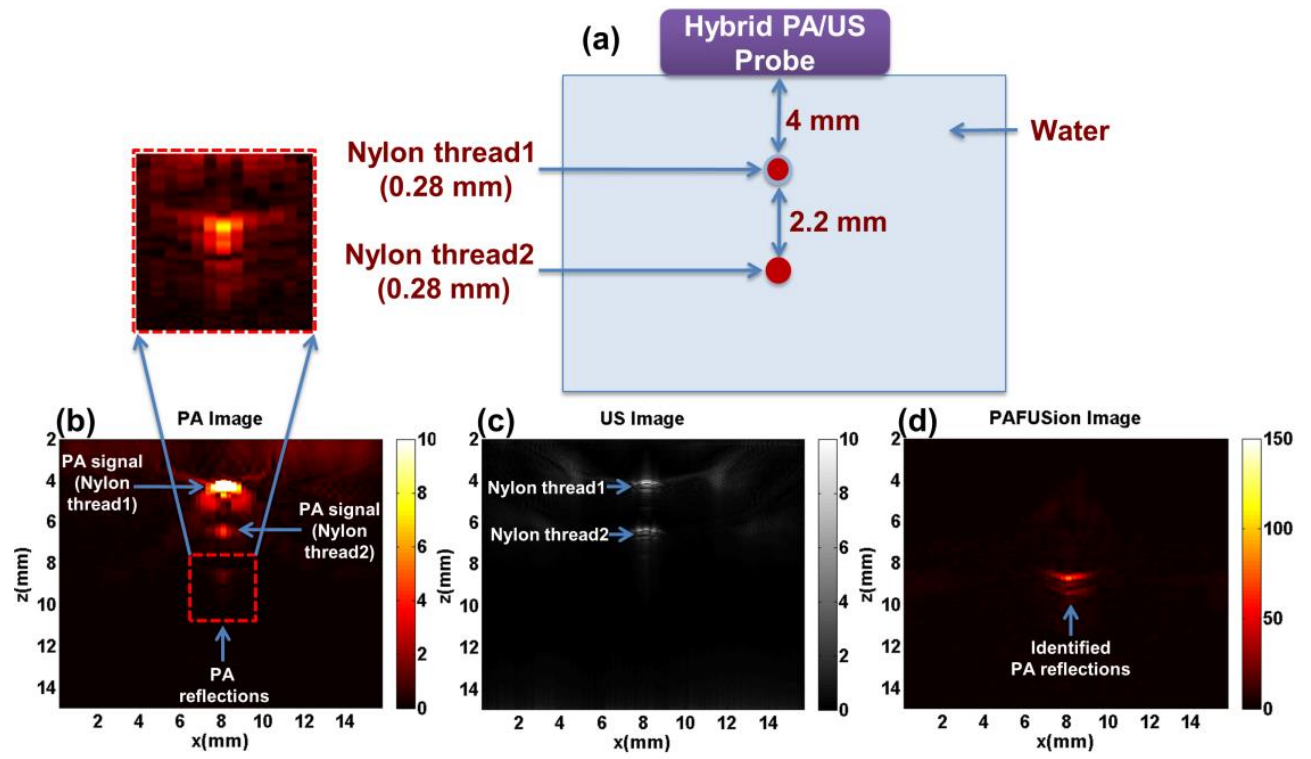

Figure 3.7 (a) Scheme of phantom 3 and experimental setup, (b) PA image and enlarged region of interest, which shows the reverberation-like details of the reflection artifacts, (c) plane wave US image, (d) PAFUSion image. 


\subsection{Discussion}

For the simulation and all phantom measurements, our results show that PAFUSion is capable of identifying all the reflection artifacts present in the PA image. Our phantom measurements led to promising results for structures separated by around $1.5 \mathrm{~mm}$, which is quite close to the clinically relevant scenario in finger joint imaging (distance between a blood vessel and a tendon, see further below). A straightforward method to identify reflection artifacts would be to use the B-mode US image and to identify potential acoustic reflectors in them, and then use this information together with simulations to identify reflections in the PA image. However, the US simulations would then rely on an imperfect input dataset, and furthermore the type of US acquisition used in the US image (center frequency, number of cycles, transmission angle) is often different from the type used in the PA image. Furthermore, the computational cost of a wave-field simulation would limit the real-time applicability of such an approach. For these reasons, it can be advantageous to physically mimic the reflection artifacts instead, by using US transmissions that match the wave field of the PA sources in a physical back-propagation approach. The key aspect in the PAFUSion process is that the zero time has to be defined at the moment when the US pulse reaches the absorber, rather than when the pulse is injected in the tissue as in normal US imaging. Hence, in the PAFUSion algorithm, the tissue itself reveals the PA reflections by applying US imaging in a manner that simulates the timing of PA images. Another critical feature in the PAFUSion process is that the US pressure distribution matches the PA initial pressure distribution. If the shapes of US and PA initial pressure distributions are not matched, it will result in wrong arrival time of the transient at different reflectors, because the wave front curvature of the diverging US wave front will be different from the PA wave front.

Any dual mode PA/US system can incorporate the PAFUSion technique without any special changes to the system. The software requirements are quite similar to those of normal line-by-line US imaging, which makes clinical implementation straightforward. There is no training required to use this technique since everything can be software controlled just as in US imaging. In this work, the PAFUSion image was obtained by weighted addition of images obtained in different steps, in which the weight is proportional to the PA signal intensity. In a variation on this procedure, the US pulse amplitude can also be varied based on the PA signal intensity. This will reduce the overhead of doing weighted addition of images during 
processing. Another practical limitation is that PAFUSion requires an extra measurement and computation step, which may make the total imaging procedure slower. However, we expect that developments in GPU (Graphical Processing Unit) and FPGA (Field Programmable Gate Array) technology can overcome this, as parallel acquisition and processing is feasible using these high-speed techniques. Speed of sound and acoustic attenuation variations in tissue may also play a major role in accuracy of this technique when applied in vivo. Future work will also focus on considering these parameters during processing.

This work was intended for presenting the proof-of-principle of the technique and therefore concentrated on a comparably simplified scenario where the reflection artifacts were present in the center part of the US imaging plane, and PAFUSion was applied on PA images containing concentrated features (nylon thread). However, the choice of the phantoms is not a limitation to the validity of the technique. Features with a more complicated shape, and features that are spatially extended are expected in an in vivo scenario. We envisage solving these in our future studies by transmitting US pulses with the shape of the identified PA feature, and then applying PAFUSion algorithm to identify reflection artifacts.

This work targeted only at identifying the reflection artifacts, but not at their elimination. Elimination of artifacts requires further investigation into a thorough calibration of pulse shape and amplitude of the focused US transmissions. Such an effort was beyond the goal of this study, but will be realized as a next step of improvement. Two critical aspects to consider for making the reflection-artifact elimination work in our next step are:

Shape of US focus: The characteristics of the US focus have an impact on the shape and intensity of identified reflection artifacts in PAFUSion. It is critical to have a narrow focus axially and laterally to mimic small PA features like blood vessels. Using a Schlierenimaging set-up, we characterized the size and shape of the US focus. When focusing US to a depth of $4.5 \mathrm{~mm}$, we achieved a focus with length of $0.2 \mathrm{~mm}$ and width $0.4 \mathrm{~mm}$ in the imaging plane. This shows that, using our system, we will be able to apply PAFUSion on small blood vessels. In the range of depth that we target (until $15 \mathrm{~mm}$ ), change in shape of the focus with respect to the depth was found to be negligible.

Frequency content: The pulse shape of the transmitted US in PAFUSion must be ideally the same as the PA pulse shape to be able to coherently subtract PA reflections and PAFUSion-identified reflections. In this study, the frequency content of the US focused at a PA feature in the PAFUSion procedure is not matched with the frequency content of the PA 
signals coming from these features. Considering the pulse width of laser diode in our integrated probe (130 ns), there may be a low pass filtering effect. Thus, the reflections in PA images are of lower frequency (4 MHz), partly outside the bandwidth of the transmitted US in PAFUSion measurements. The low frequency of the PA signals might be one of the reasons for the reflection-intensity ratio difference seen in the PA image and PAFUSion image in the phantom experiments. Matching the frequency content will be one focus of future investigation, and can be achieved either physically by adapting the US transmission spectrum, or in software by filtering.

At this point, we would like to draw attention to the side-constraints and further steps that will be important when working towards a clinical implementation of the proposed technique.

First of all, we remind the reader that PAFUSion can only identify and compensate for reflection artifacts caused by PA signals generated inside the imaging plane, but not out-ofplane clutter. This is not a strong limitation, because PAFUSion is specifically designed for a setup where the tissue is irradiated directly below the probe to maximize SNR. Normally, the resulting strong reflection artifacts inhibit the use of such a setup, and the optimum irradiation distance is determined by a trade-off between in-plane reflection artifacts and outof-plane clutter and SNR [12]. If PAFUSion manages to reduce the in-plane reflection artifacts, irradiation directly below the probe will become an option again, and then out-ofplane clutter will be insignificant.

Second, PAFUSion can only identify and compensate for the inward propagating part of PA signals that can be mimicked using the limited range of possible US transmission angles. A typical US probe has an angular aperture in the range of $-30^{\circ}$ to $30^{\circ}$, whereas typical PA sources (cylindrical blood vessels) radiate into an angle range of $360^{\circ}$, which leads to a limitation of the method. If an acoustic reflector and optical absorber are positioned side by side, then the reflection artifact caused by these will be impossible to identify using this technique. Considering the fact that most of the echo producing structures (tendon, bone) lie beneath the superficial optical absorbers that generate strong PA transients (skin, blood vessels), this limitation is not critical.

Also, when using a linear array probe, PAFUSion is limited to mimicking PA transients that propagate parallel to the imaging plane. Therefore, the probe must be oriented such that artifact-generating PA sources are oriented perpendicular to the imaging plane in order to 
obtain optimum performance. This can be easily achieved in free-hand probe guidance, and will be even less of a limitation if $1.5 \mathrm{D}$ or $2 \mathrm{D}$ arrays are used.

With increasing number of PA absorbers (skin, multiple blood vessels) and many possible reflection angles (angle between absorber to bone/tendons), identification of all the reflection artifacts may be challenging. However, it is vital to mention that an artifact reduction by $75 \%$ facilitates an increase in signal to artifact ratio by $12 \mathrm{~dB}$. Clinical PA imaging suffers from poor deep-tissue contrast because of optical attenuation and reflection artifacts, and a $12 \mathrm{~dB}$ increase in signal to artifact ratio itself will have a significant impact on image contrast.

In this work, PAFUSion was performed after manually identifying the high intensity points in the PA image. Manual identification might not be an option in a real-time application, but this is not a limitation as it is possible to automate the process of PA source identification as long as the number of strong PA sources is small. With a non-sparse distribution of PA sources, it might though be advantageous to automatically scan a large number of pixels in a region of interest in the imaging plane. To minimize the number of focused US acquisitions in view of real-time imaging, there is a possibility to use a synthetic aperture approach, where US can be synthetically focused to any desired point in the imaging plane. Instead of physically scanning e.g. $100 \times 100$ pixels with a focused beam, the focused acquisitions can be synthetically generated in software processing from e.g. just 100 plane wave transmissions using different transmit angles.

Time reversal of PA signals could also be an alternative for reducing acquisition time in comparison to separately focusing on multiple PA sources. Time reversal could be a solution for two problems: (1) the PA images often show spread-out features rather than point-like features, and (2) the different spectral contents of PA and US signals. Time reversal may lead to ingoing US signals which are spectral copies of outgoing PA signals. The only problem to which time reversal is not a solution is that of frequency dependent attenuation. Our future research will therefore focus on studying the feasibility of using the PA time reversal approach in PAFUSion. Most of the commercially available US systems may not be capable of pulsing the transducer elements with non-periodic pulses, which would be the technical challenge in using PA time reversal. This limitation can also be solved by using a synthetic approach where the US transmission pulse shape is matched with the PA signal shape using software processing. 
At this stage of PAFUSion implementation, one of the clinical applications we foresee is finger joint PA imaging. For instance, if the distance between a superficial blood vessel and a tendon is the same as the distance between the tendon and synovium, the blood vessel signal reflects on the tendon to generate reflection artifact at almost the same depth as the synovium is located. This is critical in rheumatoid arthritis imaging where inflammation of synovium is the marker. The second phantom experiment was intended to mimic this situation and it is clear that PAFUSion can be useful in these circumstances. The third phantom experiment represents the condition in which optical absorbers are by themselves acoustically reflective. These features with contrast in PA and US imaging might create problems in accurate interpretation of clinical PA images. One of the important reflection artifact sources is the skin because of its high melanin content. In any clinical applications, skin is expected to generate high PA transients, which triggers the generation of reflection artifacts. PAFUSion will help to avoid the exclusion of patients with high melanin content, from deep-tissue PA imaging. Reflection artifacts generated by small moles or hair, which is directly under the probe can also be potentially identified by using PAFUSion.

\subsection{Conclusions}

PAFUSion allows the identification of reflection artifacts in PA images by ultrasonically simulating the PA waves from the optical absorber, traversing towards the acoustic reflectors and thus by mimicking the PA reflection signals. In this proof-of-principle study, a simulation of a simple virtual phantom and three phantom experiments were performed to confirm the validity of this novel technique. Results demonstrate that PAFUSion can separate reflection signals generated inside the imaging plane from the signals of interest, and thus envisions good potential for improving PA imaging of acoustically inhomogeneous tissue. 


\subsection{References}

1. M. Xu and L.V. Wang, Photoacoustic imaging in biomedicine. Review of Scientific Instruments, 2006. 77(4).

2. P. Beard, Biomedical photoacoustic imaging. Interface Focus, 2011. 1(4): p. $602-$ 31.

3. R.G.M. Kolkman, et al., Real-time in vivo photoacoustic and ultrasound imaging. Journal of Biomedical Optics, 2008. 13(5).

4. C. Kim, et al., Performance benchmarks of an array-based hand-held photoacoustic probe adapted from a clinical ultrasound system for non-invasive sentinel lymph node imaging. Philos Trans A Math Phys Eng Sci, 2011. 369(1955): p. 4644-50.

5. K. Daoudi, et al., Handheld probe integrating laser diode and ultrasound transducer array for ultrasound/photoacoustic dual modality imaging. Optics Express, 2014. 22(21): p. 26365-26374.

6. M. Kuniyil Ajith Singh, W. Steenbergen, and S. Manohar, Handheld Probe-Based Dual Mode Ultrasound/Photoacoustics for Biomedical Imaging, in Frontiers in Biophotonics for Translational Medicine, M. Olivo and U.S. Dinish, Editors. 2016, Springer Singapore. p. 209-247.

7. M. Jaeger, J.C. Bamber, and M. Frenz, Clutter elimination for deep clinical optoacoustic imaging using localised vibration tagging (LOVIT). Photoacoustics, 2013. 1(2): p. 19-29.

8. M. Jaeger, et al., Reduction of background in optoacoustic image sequences obtained under tissue deformation. Journal of Biomedical Optics, 2009. 14(5).

9. M. Frenz and M. Jaeger, Optimization of tissue irradiation in optoacoustic imaging using a linear transducer: theory and experiments - art. no. 68561Y. Photons Plus Ultrasound: Imaging and Sensing 2008: The Ninth Conference on Biomedical Thermoacoustics, Optoacoustics, and Acoustic-Optics, 2008. 6856: p. Y8561Y8561.

10. M. Jaeger, M. Frenz, and D. Schweizer, Iterative reconstruction algorithm for reduction of echo background in optoacoustic images - art. no. 68561C. Photons Plus Ultrasound: Imaging and Sensing 2008: The Ninth Conference on Biomedical Thermoacoustics, Optoacoustics, and Acoustic-Optics, 2008. 6856: p. C8561C8561.

11. M. Jaeger, et al., Real-time clutter reduction in epi-optoacoustic imaging of human volunteers. Medical Imaging 2014: Ultrasonic Imaging and Tomography, 2014. 9040.

12. G. Held, et al., Effect of irradiation distance on image contrast in epi-optoacoustic imaging of human volunteers. Biomedical Optics Express, 2014. 5(11): p. 37653780 .

13. M.A.L. Bell, et al., Short-lag spatial coherence beamforming of photoacoustic images for enhanced visualization of prostate brachytherapy seeds. Biomedical Optics Express, 2013. 4(10): p. 1964-1977.

14. M. Jaeger, et al., Fourier reconstruction in optoacoustic imaging using truncated regularized inverse $k$-space interpolation. Inverse Problems, 2007. 23(6): p. S51S63.

15. B.E. Treeby and B.T. Cox, $k$-Wave: MATLAB toolbox for the simulation and reconstruction of photoacoustic wave fields. J Biomed Opt, 2010. 15(2): p. 021314. 
16. G. Xu, et al., Photoacoustic and ultrasound dual-modality imaging of human peripheral joints. Journal of Biomedical Optics, 2013. 18(1). 


\section{In vivo demonstration of}

\section{reflection artifact reduction in}

\section{photoacoustic imaging using}

\section{synthetic aperture photoacoustic-}

\section{guided focused ultrasound}

\section{${\text { (PAFUSion })^{3}}^{3}$}

Reflection artifacts caused by acoustic inhomogeneities are a critical problem in epi-mode biomedical photoacoustic imaging. High light fluence beneath the probe results in photoacoustic transients, which propagate into the tissue and reflect back from echogenic structures. These reflection artifacts cause problems in image interpretation and significantly impact the contrast and imaging depth. We recently proposed a method called PAFUSion (Photoacoustic-guided focused ultrasound) to identify such reflection artifacts in photoacoustic imaging. In its initial version, PAFUSion mimics the inward-travelling wavefield from small blood vessel-like PA sources by applying ultrasound pulses focused towards these sources, and thus provides a way to identify the resulting reflection artifacts. In this work, we demonstrate reduction of reflection artifacts in phantoms and in vivo measurements on human volunteers. In view of the spatially distributed PA sources that are

\footnotetext{
${ }^{3}$ This chapter has been published as: M. Kuniyil Ajith Singh, M. Jaeger, M. Frenz, and W. Steenbergen., "In vivo demonstration of reflection artifact reduction in photoacoustic imaging using synthetic aperture photoacousticguided focused ultrasound (PAFUSion)," Biomedical optics express 7(8), 2955-2972 (2016).
} 
found in clinical applications, we implemented an improved version of PAFUSion where photoacoustic signals are backpropagated to imitate the inward travelling wavefield and thus the reflection artifacts. The backpropagation is performed in a synthetic way based on the pulse-echo acquisitions after transmission on each single element of the transducer array. The results provide a direct confirmation that reflection artifacts are prominent in clinical epiphotoacoustic imaging, and that PAFUSion can strongly reduce these artifacts to improve deep-tissue photoacoustic imaging. 


\subsection{Introduction}

Photoacoustic (PA) imaging, also called optoacoustic imaging, is an emerging medical imaging modality. PA imaging is performed using short-pulsed visible or near-infrared light, which, while absorbed by molecules such as hemoglobin in blood, generates ultrasound (US) due to the thermoelastic effect. The US is measured at the surface of tissue using US detectors, and the location and spatial details of the absorber are reconstructed from the acquired US signals [1, 2]. PA imaging offers excellent optical contrast combined with ultrasonic resolution for structural and functional medical imaging. Since different lightabsorbing chromophores in tissue (hemoglobin, lipids, fat etc) have their own characteristic absorption spectrum, it is possible to distinguish these using spectroscopic PA imaging [1]. PA imaging holds promise in functional imaging of the vasculature [3], in particular for differentiating oxygen levels based on the different optical absorption characteristics of oxy and deoxy-hemoglobin [4-7]. This technique has potential to diagnose vascular diseases, inflammations and cancer at an early stage [8-12], and for monitoring the disease response to specific medications/treatments [13]. In addition to the tissue chromophores, external contrast agents functionalized for specific biochemical targets hold potential in early detection of diseases [14].

Of the different medical imaging modalities, pulse-echo US imaging is largely prevalent, considering the compactness, affordability and real-time performance for point-of-care applications. Since the detection part is the same for pulse-echo US and PA imaging, PA imaging can be easily realized in a US scanner just by adding a light delivery system and a trigger control to a commercial US-system [15]. The impact and importance of dual-mode PA/US systems for improving clinical diagnostics has been demonstrated [16-23]. Handheld dual-mode US/PA probes use reflection-mode imaging geometry (epi-mode), where optical components are directly integrated into [20] or attached to the US probe such that the light illumination is done from the same side where PA signals are detected. Bearing in mind the clinical scenario, this can help the doctors to use free-hand guidance of the probe with maximum fluence below the probe for optimal SNR. Importantly, bony and acoustically attenuating soft tissue (large breast, abdomen, limbs) can be imaged only in epi-mode as these structures obstruct propagation of acoustic waves from the irradiated tissue region to the acoustic probe [24]. 
Epi-illumination has however a downside; high light fluence beneath and proximal to the probe generates PA transients from superficial optical absorbers (i.e. melanin or blood vessels), which propagate into the tissue and reflect back from echogenic structures to produce reflection artifacts (echo clutter) [19, 24, 25]. These reflection artifacts severely limit the contrast and imaging depth [24, 26, 27]. In addition, when imaging structures which are acoustically inhomogeneous and optically absorbing, reflection artifacts may appear in the region of interest and be misinterpreted as real optically absorbing structures [25]. Also, the high intensity reflection artifacts overshadow the weak PA signals from deep tissue structures where fluence is less. These artifacts cannot be reduced by signal averaging. It has been shown in human volunteers that reflection artifacts can be minimized by irradiating the skin surface outside the imaging plane in a dark-field approach [27]. However, the benefit of such an approach is limited, and it comes at the cost of a low SNR. It is therefore not feasible with integrated systems [20] that provide pulse energies in the few $\mathrm{mJ}$ range, because they require illuminating the tissue directly below the probe to achieve a suitable SNR. For these reasons, clinically successful high-contrast PA imaging requires methods for reducing reflection artifacts.

Several methods have been studied for identifying and reducing reflection artifacts in PA imaging [24, 26, 28, 29]. LOVIT [24] and DCA [26] aim at reducing reflection artifacts that occur below a certain depth (typically below $15 \mathrm{~mm}$ ). Because they require a local tissue displacement relative to the skin surface, they are not suited for reducing artifacts close to the skin. This is especially a disadvantage with low-energy integrated systems that are designed for a shallow imaging depth, but where the in-plane irradiation results in strong reflections of the PA transient generated in the skin melanin layer. Reflections are also considered to be significant in PA tomography of small animals, and techniques for artifact reduction have been proposed for implementation in hybrid PA and US tomography systems $[30,31]$.

We recently reported on the proof-of-principle of a novel method called PAFUSion [25]. In its initial version, PAFUSion mimics the inward-travelling wavefield from small blood vessel-like PA sources by applying US pulses focused towards these sources, and thus provides a way to identify reflection artifacts. PAFUSion specifically targets at in-plane reflection artifacts, which are prominent when tissue is irradiated in-line with the US imaging plane. In this work, we demonstrate reflection-artifact-correction in addition to identification, 
towards obtaining an artifact-free PA image in quasi-real-time. In view of clinical applications where realistic PA sources can include a number of blood vessels of various sizes and extended structures such as the skin melanin layer, we implemented an improved version of PAFUSion that is more versatile than the previous focusing approach. We propose to employ synthetic backpropagation of PA signals for mimicking the inward-travelling PA field and thus the reflection artifacts caused by a complex distribution of PA sources. Backpropagation is based on reference pulse-echo US data that are obtained with elementby-element transmission. By subtracting the synthetically generated artifact signal from the detected PA signal, reflection artifacts can be significantly reduced. Because PAFUSion is equally suited for reducing superficial as well as deep reflection artifacts, it solves the limitation of previously reported approaches.

The goal of this paper is to present our first results of reflection artifact correction in PA images using improved PAFUSion. We demonstrate strong in-plane artifact reduction in phantom and in vivo measurements on forearm of human volunteers. Uncorrected epi-mode PA images and PAFUSion-corrected PA images are thoroughly compared to demonstrate the impact and importance of our method in biomedical photoacoustics.

\subsection{Theory}

PAFUSion is based on the concept that the PA wavefield can be decomposed into two parts $P_{O}$ and $P_{i}$ that propagate into opposite directional half-spaces. If the total wavefield is considered as a superposition of plane-wave components, part $P_{O}$ comprises all plane-waves with wave vectors pointing into the half-space towards the skin surface. At the time $t=0$, immediately after irradiating a hypothetical Dirac delta laser pulse but before transients start propagating, the local vibrational velocity is zero. In line with a general concept in PA imaging, each plane-wave in $P_{O}$ has therefore a counterpart with equal amplitude and wave number but opposite propagation direction. The superposition of all these counterparts constitutes the wavefield $P_{i}$. From the initial condition of zero vibrational velocity, it therefore follows that the two wavefields can be written as time-inverted aliases:

$$
P_{i}(\mathbf{r}, t)=P_{O}(\mathbf{r},-t)
$$

where $r$ denotes the spatial coordinates inside the tissue. 
Figure 4.1a illustrates the fields $P_{O}$ and $P_{i}$ that propagate towards the probe and into the tissue, respectively, for the case where skin absorption is the main source of reflection artifacts. Both $P_{O}$ and $P_{\mathrm{i}}$ are scattered by echogenic structures inside the tissue and may produce reflection artifacts. However, it is assumed that (i) mainly the part propagating into the tissue leads to significant reflection artifacts. Assumption (i) is motivated by the exponential attenuation of the irradiating laser light in epi-PA imaging, together with the rather weak US scattering of tissue: Reflection artifacts that are comparable in amplitude to the weak PA signals from inside the tissue stem from reflections of the stronger PA transients generated closer to the skin surface. Figure 4.1a illustrates the signal time traces recorded by an US array probe. They contain the directly detected PA signals from the skin and from deeper blood vessels as well as the echoes caused by back-reflection of the inwardpropagating skin PA transient. Further assumptions include:

(ii) There exists a time $T$ that fulfills following two conditions: All PA sources that are strong enough to produce significant echoes lead to a direct signal that is detected within the time interval $[0, T]$, and the earliest detected echoes arrive at $t>T$. A possible selection of $T$ is indicated in Figure 4.1a.

(iii) The aperture and the spatial sampling (element pitch) of the transducer array are sufficient to detect the full wavefield $P_{O}$.

(iv) The array elements have an isotropic Dirac delta receive and transmit impulse response, and acoustic attenuation is negligible.

If assumptions (i) to (iv) are fulfilled, re-transmission of the PA signal that was detected within the time interval $[0, T]$ leads to the reproduction of the echoes that were contained in the PA signal. Subtraction of these echoes from the original PA data results in reflectionartifact reduction. This principle is explained in more detail in the following.

The detected echoes can be modelled as the result of a linear operator $H$ acting onto the wavefield $P_{i} . H$ describes the influence of the spatial distribution of mechanical properties in a linear system theory approach, as a linear transformation from arbitrary wavefields (a function of spatial coordinate $\mathbf{r}$ and time $t$ ) to the detected echo signals (as a function of array element index $n$ and time $t$ ). For simplicity of notation, the operation of $H$ is written as a convolution. According to assumption (iv) the signal $S$ detected on elements $n$ is equal to the wavefield sampled at the element positions $\mathbf{r}_{n}$, and with the help of assumption (i) and the definition of $H, S$ can be written as: 


$$
S(n, t)=P_{O}\left(\mathbf{r}_{n}, t\right)+H(\mathbf{r}, t \rightarrow n, t) * P_{i}(\mathbf{r}, t)
$$

According to assumptions (iii) and (iv), excitation of the transducer elements with the signal $S$ after time-inversion leads to physical back-propagation of a time-inverted alias of the detected wavefield. According to assumption (ii), $S$ is identical to $P_{O}$ on the time interval $[0, T]$. Therefore, re-transmission of the signal detected during the time interval $[0, T]$ results in a time-inverted alias $P_{O}$ ' of the echo-producing part of $P_{O}$ :

$$
P_{O}{ }^{\prime}\left(\mathbf{r}, t^{\prime}\right)=P_{O}\left(\mathbf{r}, T-t^{\prime}\right)
$$

Figure $4.1 \mathrm{~b}$ illustrates the physical backpropagation for the skin absorption signal. The signal that was detected during $[0, T]$ is time-inverted and re-transmitted into the tissue. At time $t^{\prime}=T$, the wavefield $P_{O}$ ' converges to the origin of the original PA transient. For $t^{\prime}>T$ the backpropagating wavefield replicates the original inward-travelling wavefield $P_{i}$. Defining $t^{*}=t^{\prime}-T$ :

$$
P_{i}^{*}\left(\mathbf{r}, t^{*}\right) \equiv P_{O}{ }^{\prime}\left(\mathbf{r}, t^{*}+T\right)=P_{O}\left(\mathbf{r}, T-t^{*}-T\right)=P_{O}\left(\mathbf{r},-t^{*}\right)=P_{i}\left(\mathbf{r}, t^{*}\right) .
$$

$P_{i}^{*}$ is reflected in the same way as $P_{i}$, resulting in the detection of a signal $S^{*}$ that contains the same echoes as the ones that were present in the original PA signal at $t>T$ (see Figure 4.1c for illustration). Subtraction of $S^{*}$ from the original $S$ will therefore yield an echo-free PA signal. For brevity, variables $\mathbf{r}, n$ and $t$ will be omitted in $H$ :

$$
\begin{aligned}
& S^{*}\left(n, t^{*}\right)=H * P_{i}^{*}\left(\mathbf{r}, t^{*}\right)=H * P_{i}\left(\mathbf{r}, t=t^{*}\right) \\
& S(n, t)-S^{*}\left(n, t^{*}=t\right)=P_{O}\left(\mathbf{r}_{n}, t\right)+H *\left[P_{i}(\mathbf{r}, t)-P_{i}^{*}\left(\mathbf{r}, t^{*}=t\right)\right] \\
& =P_{O}\left(\mathbf{r}_{n}, t\right) .
\end{aligned}
$$

Whereas an isotropic Dirac delta impulse response was assumed so far (assumption iv), typical clinical probes have a limited bandwidth and angle-dependent transmit and receive characteristics. This is not a strong limitation to the technique, because differences in the transducer impulse responses for PA detection and US transmission/detection can be compensated for by filtering operations. To allow detection and backpropagation of arbitrary wavefields according to assumptions (iii) and (iv), a probe would require an infinite 2D 
aperture and an omnidirectional receive and transmit response. In reality, the limited probe aperture and the finite element pitch, together with a limited directional response, do not allow detecting and imitating arbitrary wavefields. For that reason, backpropagation is only applicable within further constraints to $P_{O}$ and thus to the geometry of optical absorbers. In the case of blood vessels or point-like absorbers, the laterally propagating part of the transients cannot be detected and thus reflection artifacts generated by these transients cannot be mimicked via backpropagation. In a similar way as for assumption (i), one may argue that the most significant echoes will be produced by the axially propagating part of the transients. When using a linear probe, only transients propagating parallel to the imaging plane can be mimicked. Such transients are generated by cylindrical or flat tissue structures (such as e.g. blood vessels, the skin melanin layer) when they are oriented perpendicular to the imaging plane.
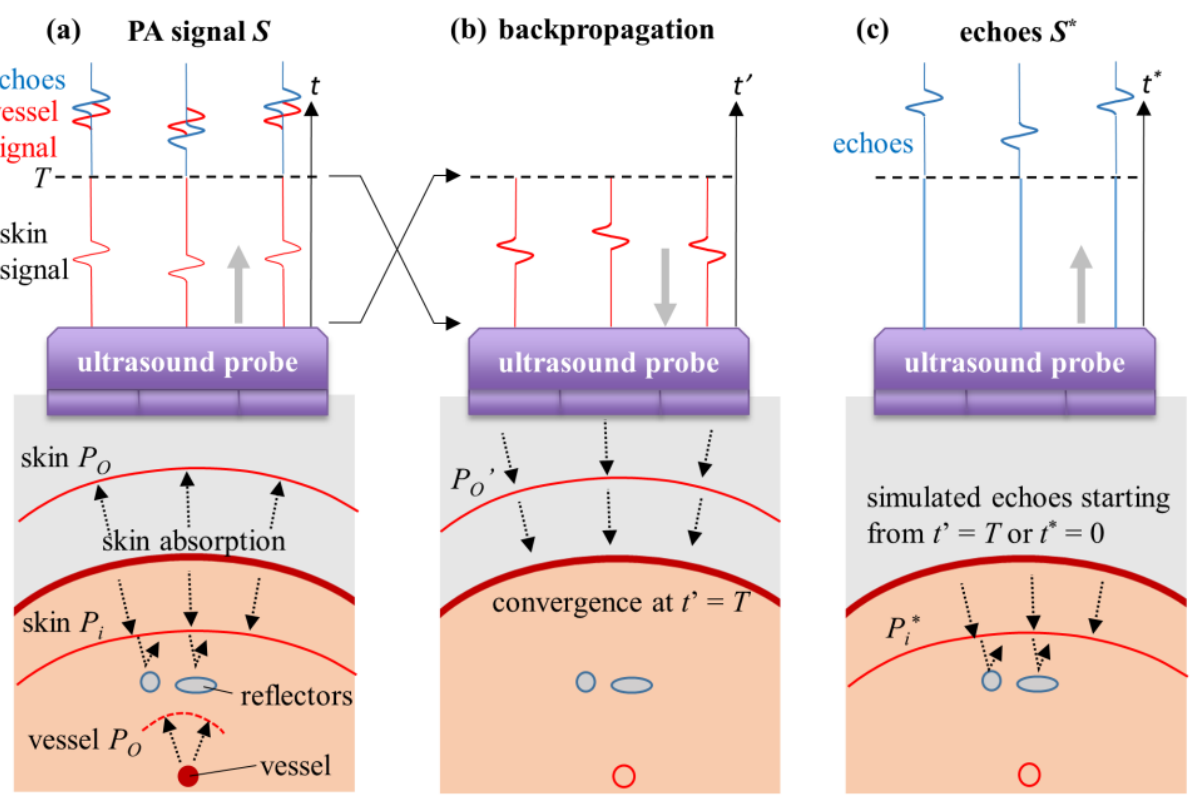

Figure 4.1 (a) Illustration of inward $\left(P_{i}\right)$ and outward-propagating $\left(P_{O}\right)$ PA transients, for the special case where they are generated by optical absorption in the skin melanin layer. (b) Time-inversion and backpropagation of the PA signal from interval $t=[0, T]$, towards (c) mimicking the inward-travelling wavefield $P_{\mathrm{i}}^{*}$ and identification of resulting echoes. 
Taking into account these experimental limitations, the less general statement of equation(4.6) is that echoes can be reduced within the systems frequency bandwidth, and depending on proper alignment of the probe relative to the reflection artifact-generating tissue structures.

\subsubsection{Synthetic PA backpropagation based on reference pulse-echo data}

To avoid motion artifacts, physical backpropagation requires immediate re-transmission of detected PA signals. To be able to do so, an ideal PAFUSion system would require the frontend hardware facilities for recording PA signals, performing the filter operations for transducer impulse response matching, and re-transmitting arbitrary waveforms. In a typical state-of-the-art system, however, these facilities will not be available. Instead of a physical backpropagation, we therefore propose a synthetic approach. This approach is based on postprocessing pulse-echo acquisitions that are obtained after transmitting on single elements at a time. These data can be acquired in immediate succession with PA data with a minimum time delay. The concept of synthetic PA backpropagation using element-wise pulse-echo acquisitions is based on writing the backpropagated wavefield $P_{i}{ }^{*}$ as a superposition of the wavefields $P_{i, n}^{*}$ produced by back-propagation from single elements $n$. The wavefield produced by each single element can in turn be written as a convolution of the time-inverted PA signal $S$ detected on that element, with the wavefield $P_{\delta, n}$ produced by transmission of a Dirac delta pulse on that element:

$$
P_{i}^{*}\left(\mathbf{r}, t^{*}\right)=\sum_{n} P_{i, n}{ }^{*}\left(\mathbf{r}, t^{\prime \prime}=t^{*}+T\right)=\sum_{n} \int_{0}^{T} d t^{\prime} S\left(n, T-t^{\prime}\right) \cdot P_{\delta, n}\left(\mathbf{r}, t^{\prime \prime}-t^{\prime}\right) .
$$

Correspondingly, the echoes $S^{*}$ detected upon backpropagation of $P_{i}{ }^{*}$ can be written as a superposition of the echoes $S_{n}^{*}$ detected on elements $n^{*}$ after backpropagation of the wavefields $P^{*}{ }_{i, n}$ from elements $n$. This in turn can be written as a convolution of the echoes $S_{\delta, n}$ detected after transmitting Dirac delta pulses on elements $n$, convolved with the timeinverted signal from the respective same element: 


$$
\begin{gathered}
S^{*}\left(n^{*}, t^{*}\right)=\sum_{n} S_{n}^{*}\left(n^{*}, t^{\prime \prime}=t^{*}+T\right)=\sum_{n} \int_{0}^{T} d t^{\prime} S\left(n, T-t^{\prime}\right) \cdot\left[H * P_{\delta, n}\right]\left(n^{*}, t^{\prime \prime}-t^{\prime}\right) \\
\equiv \sum_{n} \int_{0}^{T} d t^{\prime} S\left(n, T-t^{\prime}\right) \cdot S_{\delta, n}\left(n^{*}, t^{\prime \prime}-t^{\prime}\right) .
\end{gathered}
$$

Therefore, backpropagation can be performed in a synthetic way using the following algorithm:

- $\quad$ Record pulse-echo signals $S_{\delta,{ }_{n}}\left(n^{*}, t^{\prime \prime}\right)$ on elements $n^{*}$ after transmission of Dirac delta pulses on each element $n$

- $\quad$ Time-invert the PA signals $S(n, t)$ detected during the interval $t=[0, T]$

- $\quad$ Numerically convolve the time inverted signals with the signals $S_{\delta, n}\left(n^{*}, t^{\prime \prime}\right)$, shift in time according to $t^{*}=t^{\prime \prime}-T$

- $\quad$ Superimpose the results to form $S^{*}\left(n^{*}, t^{*}\right)$

The reference pulse-echo data acquired for synthetic backpropagation can be a collection of acquisitions on all elements $n^{*}$, after transmissions on elements $n$ as described so far. Alternatively, synthetic backpropagation can be based on any other collection of US data that provides sufficient information, such as a collection of plane-wave acquisitions with different transmit steering angles.

\subsection{Materials and methods}

\subsubsection{Equipment and setup}

For the phantom and in vivo studies, an epi-mode combined PA and US system was used as shown in Figure 4.2. Image acquisition was implemented on a Vantage 64 LE data acquisition system (VDAS, Verasonics Inc., Redmond, WA), which can read data from 64 channels at a time, and perform continuous data transfer for real-time processing on a host PC via a pci-e interface (6.6 GByte/s sustained transfer rate). The processing and the order in which different software scripts are executed are explained in section 4.3.2. The US probe used for the experiment was a linear array transducer (ATL L7-4, Philips N.V., NL), containing 128 elements at a pitch of $0.3 \mathrm{~mm}$ with a center frequency of $5 \mathrm{MHz}$ and fractional bandwidth of $80 \%$. For avoiding reflection artifacts caused by optical absorption in the rubber material of the transducer aperture, the aperture was optically shielded with a thin aluminum foil 
acoustically coupled with US gel. A Q-switched Nd:YAG laser (Quanta Ray, Newport, CA) provided pulsed laser light at a wavelength of $1064 \mathrm{~nm}$ with a pulse duration of $10 \mathrm{~ns}$ and a repetition rate of $10 \mathrm{~Hz}$. The output of the laser was coupled into an $8 \mathrm{~mm}$ diameter optical fiber bundle (Fiberoptics, Switzerland), with a rectangular distal fiber terminal (20 mm x 1 $\mathrm{mm}$ ) aligned parallel to the linear transducer array.

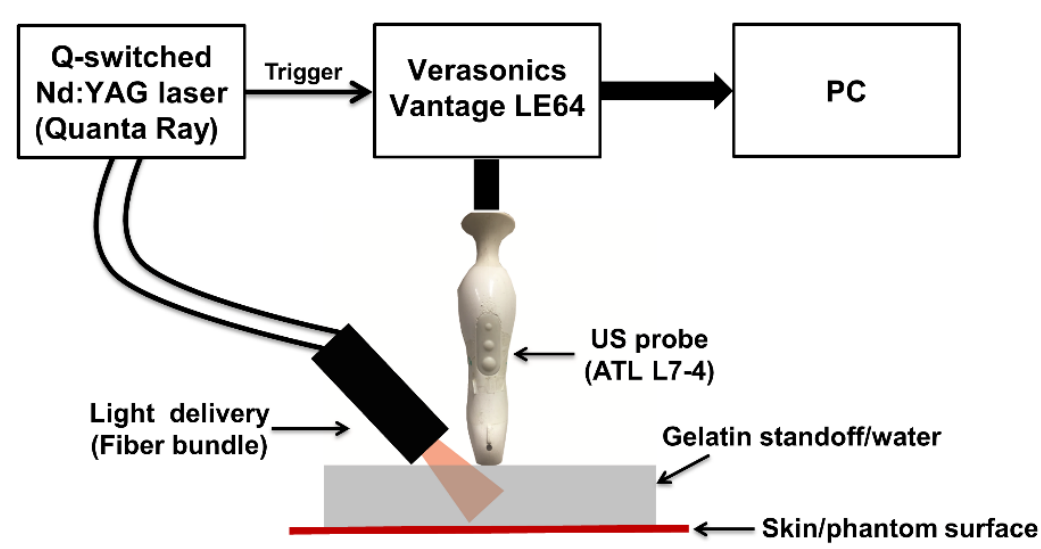

Figure 4.2 Illustration of the experimental setup.

In all experiments, the output angle of the fiber terminal was aligned in such a way that the location of main optical absorption coincided with the transducer's imaging plane. On one hand, this irradiation geometry resulted in high fluence below the US probe and thus optimum PA signal-to-noise ratio. On the other hand, it was required to make sure that reflection artifacts were generated only by PA transients that could be simulated by US transmission. The area of illumination on the skin was $3 \mathrm{~mm}$ by $23 \mathrm{~mm}$, resulting in a radiant exposure of $70 \mathrm{~mJ} / \mathrm{cm}^{2}$ which was well below the limit of the maximum permissible exposure (MPE) for skin $\left(100 \mathrm{~mJ} / \mathrm{cm}^{2}\right.$ at $\left.1064 \mathrm{~nm}\right)$.

\subsubsection{Experimental implementation of the synthetic backpropagation technique}

A scan sequence script for defining the acquisition of PA and US data by the VDAS as well as post-processing routines for synthetic backpropagation and image display on the host PC were programmed in Matlab. The Verasonics sequence executor (VSX) software executed the scan sequence and post-processing. 
In a first step, the scan sequence defined the acquisition of pulse-echo data on all elements per transmission on each single element. To minimize acquisition time and thus the influence of tissue motion, all data were buffered on the VDAS' local memory before transferring to a first buffer frame on the host PC. In a second step, PA data were acquired and transferred to a second buffer frame. As a third step, for creating memory for storing the PAFUSion data to be obtained later (see below), a dummy PA acquisition was defined in the scan sequence, without a preceding laser pulse but with all other specifications identical to the real PA acquisition. An external function performed the synthetic backpropagation of the PA data (second frame) according to the convolution approach in equation(4.8), based on the elementwise pulse-echo data (first frame). When acquiring the pulse-echo data, care was taken to use a low transmit voltage and to make sure that the signal amplitude was well within the range of the analog-to-digital converters, because linearity of propagation and detection is an important requirement for employing the synthetic backpropagation approach.

The transducer's two-way impulse response distorts the waveform of the pulse-echo data in comparison to the detected PA data. To match the waveforms of the synthetically generated and the real echo signals - and thus enable accurate subtraction $-\mathrm{S}$ and $\mathrm{S}^{*}$ were convolved with a matched pair of finite impulse response (FIR) filters, one for each type of signal. These filters were calibrated prior to the experiments in a simple setup with a plane steel reflector. An isotropic transmit and receive characteristic was assumed to be able to restrict filtering to the time dimension. The calibration measurements demonstrated that this approximation was roughly valid up to $\pm 20^{\circ}$ transmit/receive angle, which was sufficient for synthetic backpropagation in the proposed experiments. Waveform matching over a larger angle range requires plane-wave decomposition of detected signals for different receive directions, which would significantly increase the numerical burden.

The resulting synthetic data (PAFUSion data) was then transferred into the third buffer frame that was defined within the dummy PA acquisition. After that, PA image reconstruction was performed both on the second data frame (real PA image) and on the third data frame (PAFUSion image) using the Verasonics proprietary pixel-based reconstruction algorithm. In addition, the intensity of the PAFUSion image was scaled to match the PA image intensity. The identified reflection artifacts (PAFUSion image) were then subtracted from the PA image to obtain the corrected PA image. The corrected PA image was displayed along with the PA and PAFUSion image. The subtraction was performed on the reconstructed 
RF data prior to envelope detection, but the images were finally displayed as linear square envelope. In addition to the PA images, a synthetic aperture US image was reconstructed from the first frame data. For presentation in this paper, all the final results were additionally reconstructed offline from saved raw data by using frequency domain reconstruction algorithms [32].

The whole procedure can in principle be performed in real-time, limited only by the laser pulse rate $(10 \mathrm{~Hz})$. The signal acquisition speed for the element-wise pulse-echo data was similar to a conventional line-by-line scan. Two acquisitions (each on 64 elements) for the full probe aperture per transmitting element, times 128 for all transmitting elements, resulted in a total number of 256 acquisitions. With a $40 \mu$ s round-trip time for $30 \mathrm{~mm}$ imaging depth, the minimum acquisition time for a full data set was roughly $10 \mathrm{~ms}$, and a sampling rate of $20 \mathrm{MHz}$ resulted in a data size of 12.8 Msamples and thus an estimated data transfer time of roughly $4 \mathrm{~ms}$, considering the system's 14 bit sample size (roughly 2 Bytes) and transfer rate (6.6 Gbyte/s). Using the Verasonics proprietary pixel-based algorithm, reconstruction of the three images (US, PA, PAFUSion) required negligible time. The speed bottleneck in the current implementation of the technique was the synthetic backpropagation that was coded in Matlab, limiting the frame rate to one image every three seconds. Speed may be significantly increased and made real-time when using parallel processing, but this was not yet implemented in the present study. The quasi- real-time display was still helpful because it provided a visual feedback for aligning the imaging plane for optimum artifact reduction.

\subsubsection{Phantom experiment}

Figure 4.3 shows the schematic of the phantom and the setup used for the validation of synthetic backpropagation PAFUSion. The phantom consisted of one optical absorber (brown nylon thread, $0.28 \mathrm{~mm}$ diameter) positioned above an acoustic reflector (Delrin rod, $1.9 \mathrm{~mm}$ diameter). Both the optical absorber and acoustic reflector were placed inside a container filled with water, in such a way that they were perpendicular to the imaging plane of the US transducer that was immersed in the water. This phantom was used to study a comparatively simple scenario. No optical scattering agent was added to the water, to maximize the amplitude of the PA transient (and thereby of the PA reflection). In this experiment, the time $T$ was selected in such a way that the PA signal from the thread and the delrin rod was backpropagated. 


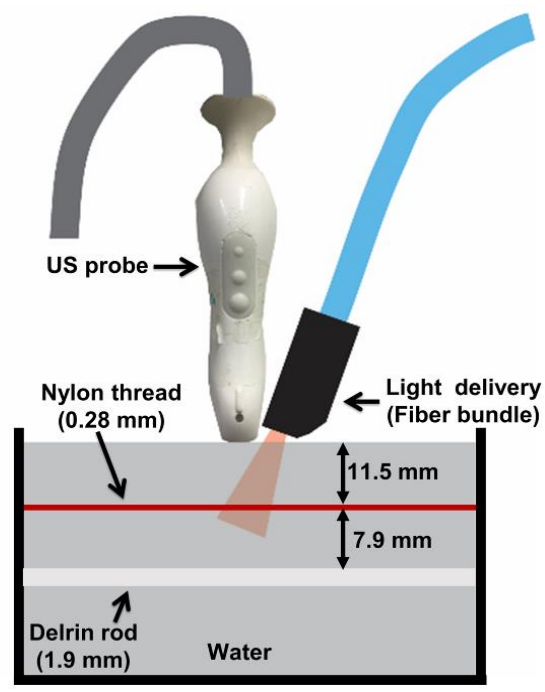

Figure 4.3 Scheme of the phantom and experimental setup.

\subsubsection{In vivo measurements on human forearm}

The ability of PAFUSion to identify and eliminate reflection artifacts in vivo was investigated by measurements on a volunteer's right forearm. Considering the large number of optical absorbers (melanin, veins, arteries etc.) and acoustic reflectors (skin, muscle layers, fascia) in this area, one can expect a complex variety of reflection artifacts. To facilitate optical irradiation at the intersection of the skin and the imaging plane below the transducer aperture, we designed a gelatin stand-off pad (Figure 1.4) that provided acoustic coupling over a distance of $15 \mathrm{~mm}$ in-between the forearm and the US probe. To minimize tissue motion inbetween successive frames at the rather low frame rate while aligning the probe, the forearm was immobilized inside an arm holder as shown in Figure 1.4. The US probe was aligned with the imaging plane perpendicular to the forearm and rigidly mounted along with the optical fiber bundle using a mechanical stage. Measurements were done at two different locations on the forearm, where reflection artifacts were well visible in PA images. The quasi real-time display of PA images provided feedback to aid selection of locations for measurements. In all in vivo measurements, $\mathrm{T}$ (time until which only real PA signals were expected) was selected in such a way that the PA signal from only the skin and the superficial blood vessels until $2 \mathrm{~mm}$ depth were synthetically backpropagated for PAFUSion. 


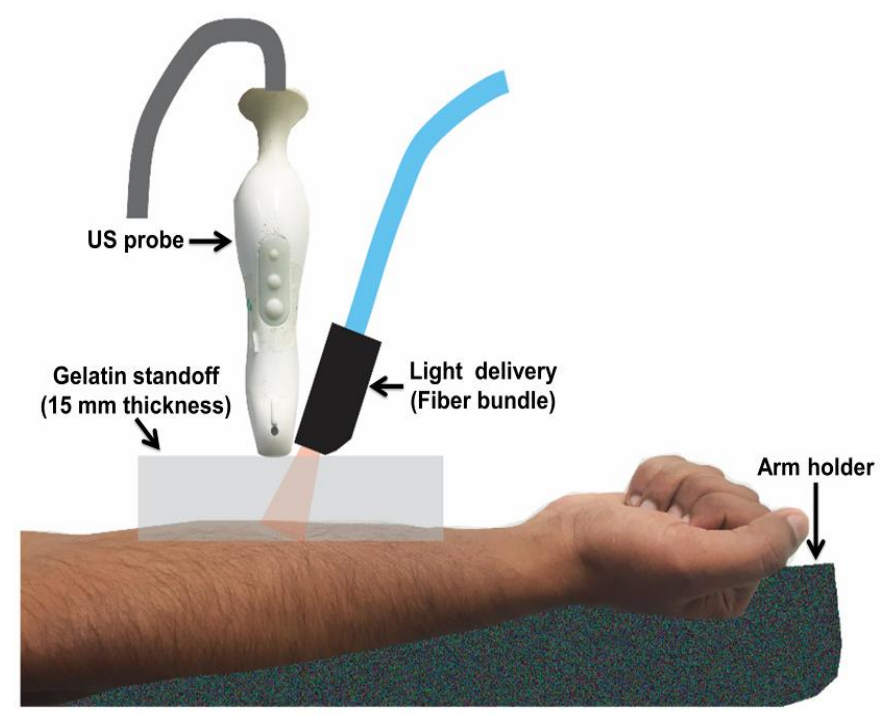

Figure 4.4 Epi-mode PA/US setup used for the in vivo measurements.

\subsubsection{Quantitative analysis of reflection-artifact-reduction}

The efficiency of PAFUSion in reducing reflection artifacts was quantitatively assessed by offline processing. For this purpose, the pixel intensities of selected regions of interest (ROI) in the image were compared, before and after correction of PA images using PAFUSion. Regions of interest were selected based on the brightness of the features in the uncorrected PA image. All features with image intensities above a certain threshold were considered for the comparison. Few of them are indicated and discussed in the Results section. The pixel intensity of a ROI was calculated as the average intensity of 9 pixels ( 3 by 3 ) centred around the maximum intensity value in the area. For comparison, pixel intensities of the same selected regions were determined in the uncorrected $\left(\mathrm{PA}_{\mathrm{i}}\right)$ and in the corrected PA image $\left(\right.$ CorrPA $\left._{i}\right)$. The ratio of $\mathrm{PA}_{i}$ and CorrPA $A_{i}$ (Intensity reduction ratio $=\mathrm{PA}_{\mathrm{i}} / \mathrm{CorrPA}_{\mathrm{i}}$ ) was calculated and expressed in $\mathrm{dB}$ to highlight the potential of PAFUSion in reducing reflection artifacts.

\subsection{Results}

In all images, lateral and axial coordinates are represented by $\mathrm{x}$ and $\mathrm{z}$ respectively. The square envelopes of the images (PA, US, PAFUSion and corrected PA) are displayed in linear scale. 


\subsubsection{Phantom experiment}

Figure 4.3 shows a schematic of the phantom and the orientation of US probe in the experimental setup. This phantom contained one optical absorber (nylon thread) and an acoustic reflector (Delrin rod). Both the nylon thread and the Delrin rod are visible in the US image (Figure 4.5b). Figure 4.5a shows the reconstructed PA image in which the nylon thread (marked as $\mathrm{a}_{1}$ at $\mathrm{x}=20 \mathrm{~mm}, \mathrm{z}=11.5 \mathrm{~mm}$ ) and the Delrin rod (marked as $\mathrm{a}_{2}$ at $\mathrm{x}=20 \mathrm{~mm}, \mathrm{z}$ $=19.5 \mathrm{~mm}$ ) are visible. The wing-like artifacts near the nylon thread are grating lobes, caused by the rather large element pitch $(0.298 \mathrm{~mm})$ in comparison to the acoustic wavelength at the center frequency of the transducer. They are more apparent in the PA image than in the US image owing to the different color map. Visibility of the Delrin rod in the PA image can be explained by optical absorption on its surface. In an ideal case, only the optically absorbing structures are expected to show up in the reconstructed PA image. However, a further feature is visible in the PA image at a depth around $\mathrm{z}=27 \mathrm{~mm}$ (marked as $\mathrm{a}_{3}$ ).

The PAFUSion image (Figure 4.5c) evidently revealed that this feature is a reflection artifact, since shape and spatial details of the reflection generated by PAFUSion matches with the real PA feature. It is worth noticing that the PAFUSion image also suggests that reflection artifacts were present at the locations of the nylon thread and Delrin rod. Even though such reflection artifacts would not be distinguishable from the nylon thread/Delrin rod in the PA image, they may actually represent reality: Part of the PA transient that is generated at the thread/Delrin rod surface may be caused by heating of the adjacent water via heat conduction from the surfaces of the thread/Delrin rod. This part would then echo back from the nylon thread/Delrin rod itself, producing reflection artifacts at the location of generation of the PA transients. More likely, however, these artifacts may simply be the result of reflections of the backpropagated wavefield at the absorber surface, immediately before it converges at $t^{*}=0$ to inside the absorber where the original PA transient was generated. Because these reflections occur before the hypothetical time zero, they do not correspond to real PA reflections but are an artifact of the backpropagation technique. In agreement with assumptions (i) and (ii), such echoes are not significant compared to the amplitude of the actual PA signal and thus could be omitted from the theoretical derivation. They are, however, noticeable when looking at the PAFUSion image alone. Similarly, echoes can already be generated when the backpropagated wavefield interacts with more superficial 
structures. The amount of such artifacts will therefore depend on the choice of $T$. The longer the backpropagated signal, the more false echoes can occur before $t^{*}=0$ is reached.
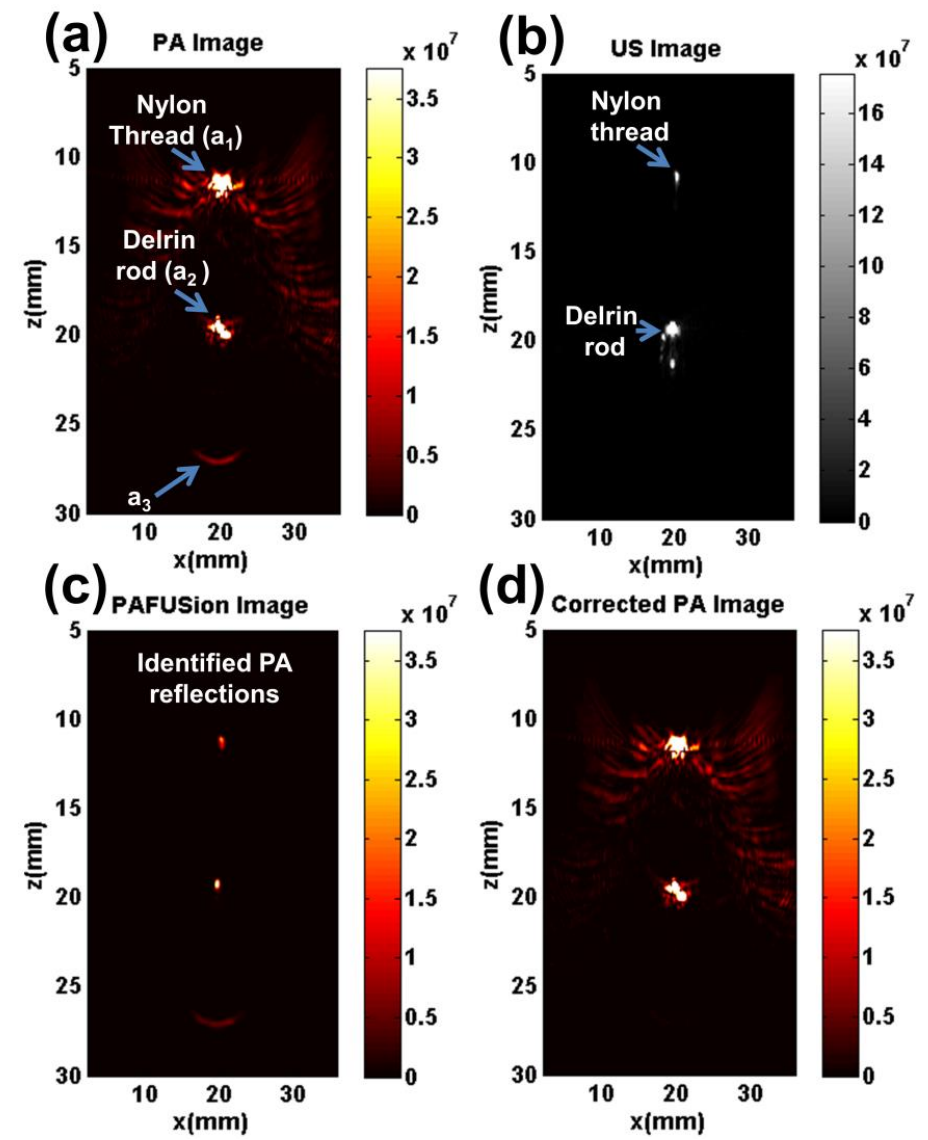

Figure 4.5 Phantom experiment: (a) PA image, (b) US image, (c) PAFUSion image with identified reflection artifacts, (d) corrected PA image.

Figure $4.5 \mathrm{~d}$ shows the corrected PA image obtained by subtracting the PAFUSionidentified reflection artifacts from the real PA image. It is evident that the reflection artifacts are strongly reduced by using PAFUSion. The pixel intensities of three regions marked in the PA image as $a_{1}, a_{2}$ and $a_{3}$ were compared with the same regions in the corrected PA image. The reflection artifact $\left(\mathrm{a}_{3}\right)$ intensity was found to be reduced by $12 \mathrm{~dB}$. On the other hand, the intensities of the features $\mathrm{a}_{1}$ and $\mathrm{a}_{2}$ were reduced by only $0.005 \mathrm{~dB}$ and $0.35 \mathrm{~dB}$ respectively. These features can therefore correctly be assumed to be real PA sources. The 
slight reduction in PA signal of $\mathrm{a}_{1}$ and $\mathrm{a}_{2}$ resulted from the reflection artifacts that were identified (potentially wrongly) at the respective same locations. It is important to mention that this signal reduction - whether caused by true echoes or just by an artifact of the backpropagation - would always be proportional to the real signal. It will therefore not affect the relative amplitude of the signal, e.g. when scanning the tissue with multiple optical wavelengths for spectral PA imaging.

\subsubsection{In vivo measurements on human forearm}

Figure 4.6a shows one PA image out of the volunteer study (in vivo measurement 1 ). The orientation of the US probe and the forearm is indicated in Figure 4.4. Figure 4.6e shows the same PA image, but with most of the various distinct features marked for reference. The skin signal ( $\mathrm{a}_{9}$ ) caused by the melanin is evident as a bright line at $\mathrm{z}=15 \mathrm{~mm}$. Three bright features $\left(\mathrm{a}_{10}, \mathrm{a}_{11}\right.$, and $\left.\mathrm{a}_{12}\right)$ are visible just underneath the skin signal, which may be three superficial blood vessels. Apart from these, the PA image contains plenty of diffuse features ( $a_{1}$ to $a_{8}$ ) just below the skin and deeper inside the tissue. Figure 4.6b shows the synthetic aperture US image that contains many echogenic structures. The skin surface is visible as a specular reflection at $\mathrm{z}=15 \mathrm{~mm}$. Acoustic reflections from tissue structures inside the subcutaneous fat layer are visible as dotted echogenic features below the skin layer. The extended lines deep in the image at $\mathrm{z}=18 \mathrm{~mm}$ to $20 \mathrm{~mm}$ are the epimysia between different muscles.

Figure 4.6c shows the PAFUSion image that shows only the simulated reflection artifacts. Our method demonstrates that the majority of the features in the PA image correlate with the simulated reflections. Except for $\mathrm{a}_{9}, \mathrm{a}_{10}$, and $\mathrm{a}_{11}$, all other features in the PA image ( $\mathrm{a}_{1}$ to $\mathrm{a}_{8}$ ) are thus identified as reflection artifacts by the PAFUSion image. Similar to the phantom results, a reflection artifact was identified at the depth of the melanin layer itself (line like feature at around $\mathrm{z}=15 \mathrm{~mm}$ ). This may again correspond to a real reflection, as skin is optically absorbing as well as acoustically reflecting, or it may simply be an artifact of the technique in analogy to what was hypothesized in the phantom experiment. The amplitude of such reflections is significantly smaller than the amplitude of the PA signal and may be neglected under many circumstances. If not negligible, it is proportional to the PA signal. Therefore, the relative variation of the PA amplitude with wavelength will be unaltered in the corrected PA image, an important prerequisite for e.g. oxygen saturation determination. 

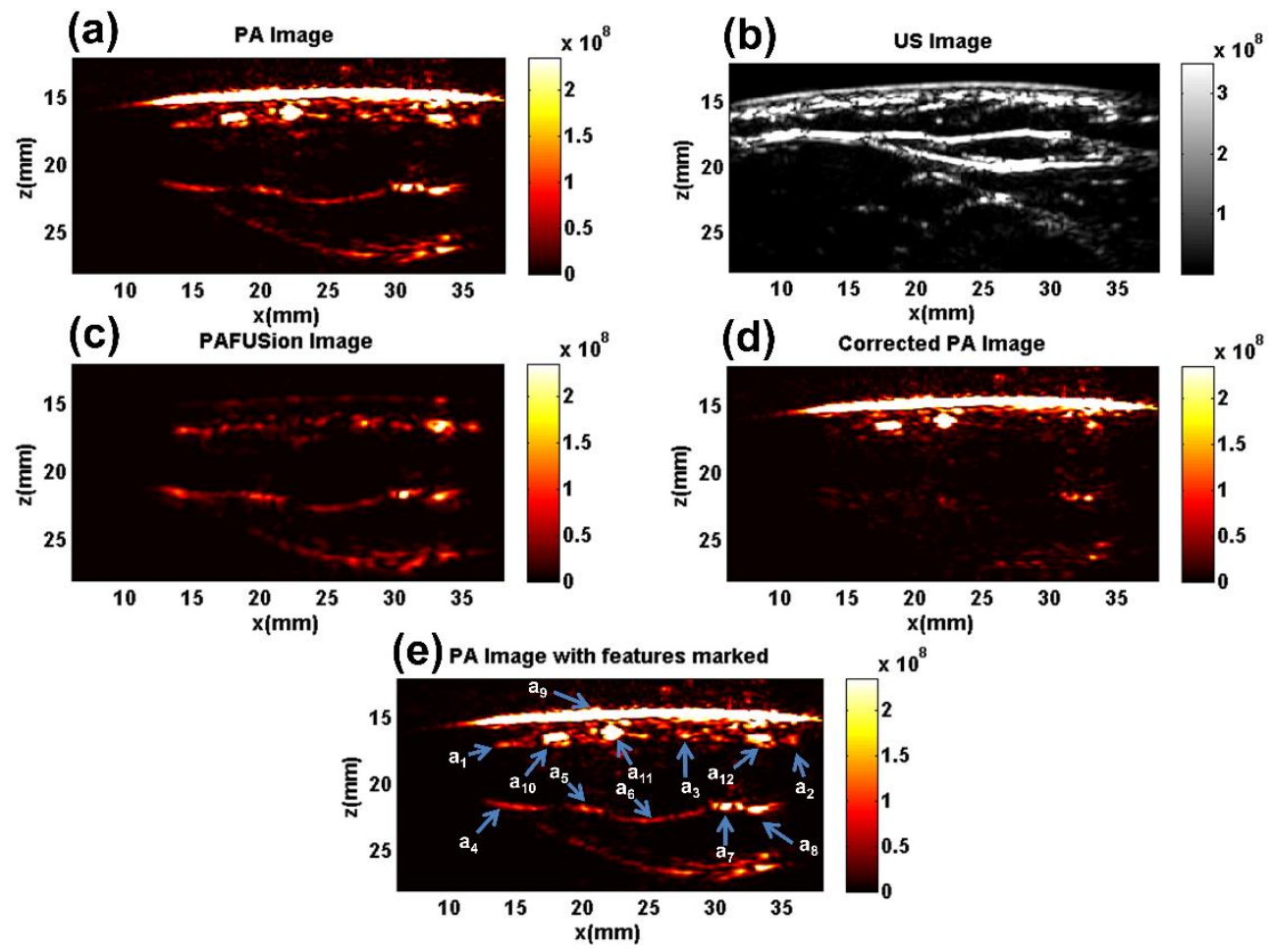

Figure 4.6 In vivo measurement 1: (a) PA image, (b) US image, (c) PAFUSion image with identified reflection artifacts, (d) corrected PA image after subtraction of reflection artifacts, (e) PA image with features marked for reference.

Figure 4.6d shows the corrected PA image obtained after subtracting the PAFUSion image from the PA image. All the identified reflection artifacts are strongly reduced in the corrected PA image. The remaining features in the corrected PA image are $a_{9}, a_{10}, a_{11}$. The feature $\mathrm{a}_{12}$ is of special interest: In PAFUSion image, a much brighter signal is visible exactly at the same location as $\mathrm{a}_{12}$, but this feature persists in the corrected PA image. This feature might therefore well be a blood vessel that was initially mixed with a reflection artifact from which it was recovered. Two features visible at depth in the corrected PA image $(x=32 \mathrm{~mm}$, $\mathrm{z}=22 \mathrm{~mm}$ and $\mathrm{x}=33 \mathrm{~mm}, \mathrm{z}=26 \mathrm{~mm}$ ) may be deeper blood vessels, which were recovered from surrounding reflection artifacts, but could also be the result of inaccurate image subtraction. It is especially worth mentioning that PAFUSion identified most of the PA features located directly below the skin as reflections that would normally be interpreted as 
real PA signals. Depending on the clinical applications, it may be of paramount importance to get rid of these artifacts for accurate image interpretation.

The ratio of PA image intensity before and after correction was calculated for all the regions marked in the PA image. These values are summarized in $\mathrm{dB}$ scale in Table 4.1 to quantitatively validate the efficiency of PAFUSion in reducing reflection artifacts.

\begin{tabular}{|c|c|c|c|c|c|c|c|c|c|c|c|c|}
\hline $\begin{array}{c}\text { Region } \\
\text { of } \\
\text { interest }\end{array}$ & $\mathbf{a}_{\mathbf{1}}$ & $\mathbf{a}_{2}$ & $\mathbf{a}_{3}$ & $\mathbf{a}_{\mathbf{4}}$ & $\mathbf{a}_{\mathbf{5}}$ & $\mathbf{a}_{\mathbf{6}}$ & $\mathbf{a}_{7}$ & $\mathbf{a}_{\mathbf{8}}$ & $\mathbf{a}_{9}$ & $\mathbf{a}_{10}$ & $\mathbf{a}_{11}$ & $\mathbf{a}_{12}$ \\
\hline $\begin{array}{c}\text { IRR } \\
(\mathbf{d B})\end{array}$ & 8.5 & 14 & 18 & 9 & 13 & 11 & 22 & 15 & 0.6 & 0.4 & 0.5 & 7 \\
\hline
\end{tabular}

Table 4.1 Intensity reduction ratio (IRR) of all the marked regions in the PA image for in vivo measurement 1.

Table 4.1 demonstrates that the intensities of features $a_{1}$ to $a_{8}$ (reflection artifacts) are significantly reduced in the corrected PA image, with an average reduction of around $13 \mathrm{~dB}$. The intensities of features $\mathrm{a}_{9}, \mathrm{a}_{10}$, and $\mathrm{a}_{11}$, were reduced by around $0.5 \mathrm{~dB}$ which is significantly less than what was measured for $\mathrm{a}_{1}$ to $\mathrm{a}_{8}$, substantiating the hypothesis that these were real PA sources. The intensity of $\mathrm{a}_{12}$, which is still well visible in the corrected image, is reduced by $7 \mathrm{~dB}$ which is in the middle $\mathrm{dB}$ range, supporting the assumption that this was a real PA source which was initially mixed with a reflection artifact.

Figure 4.7a shows the PA image from a slightly different location on the same forearm (in vivo measurement 2). Figure 4.7e shows the same PA image, but with several selected bright features $\left(a_{1}-a_{9}\right)$ marked for reference. The skin signal is visible as a bright arc at a depth of around $16.5 \mathrm{~mm}\left(\mathrm{a}_{5}\right)$. The double layered feature just below the skin $\left(\mathrm{a}_{7}\right)$ may be a superficial blood vessel. Several other bright features are visible in the PA image. Among them are: 1) A blob-like structure $\left(\mathrm{a}_{6}\right)$ below the skin and in proximity to $\mathrm{a}_{7} ; 2$ ) a prominent double layered feature $a_{9}$ and 3 ) the extended line-like structure $\left(a_{8}\right)$ close to $a_{9}$. Several other features are visible in the PA image out of which a few are marked in Figure 4.7e $\left(a_{1}-a_{4}\right)$. In such a scenario, it is difficult to differentiate between real PA features and reflection artifacts. Similar to the in vivo measurement 1,Figure $4.7 \mathrm{~b}$ shows the synthetic aperture US image in which different echogenic structures are visible that may lead to PA reflection artifacts. 

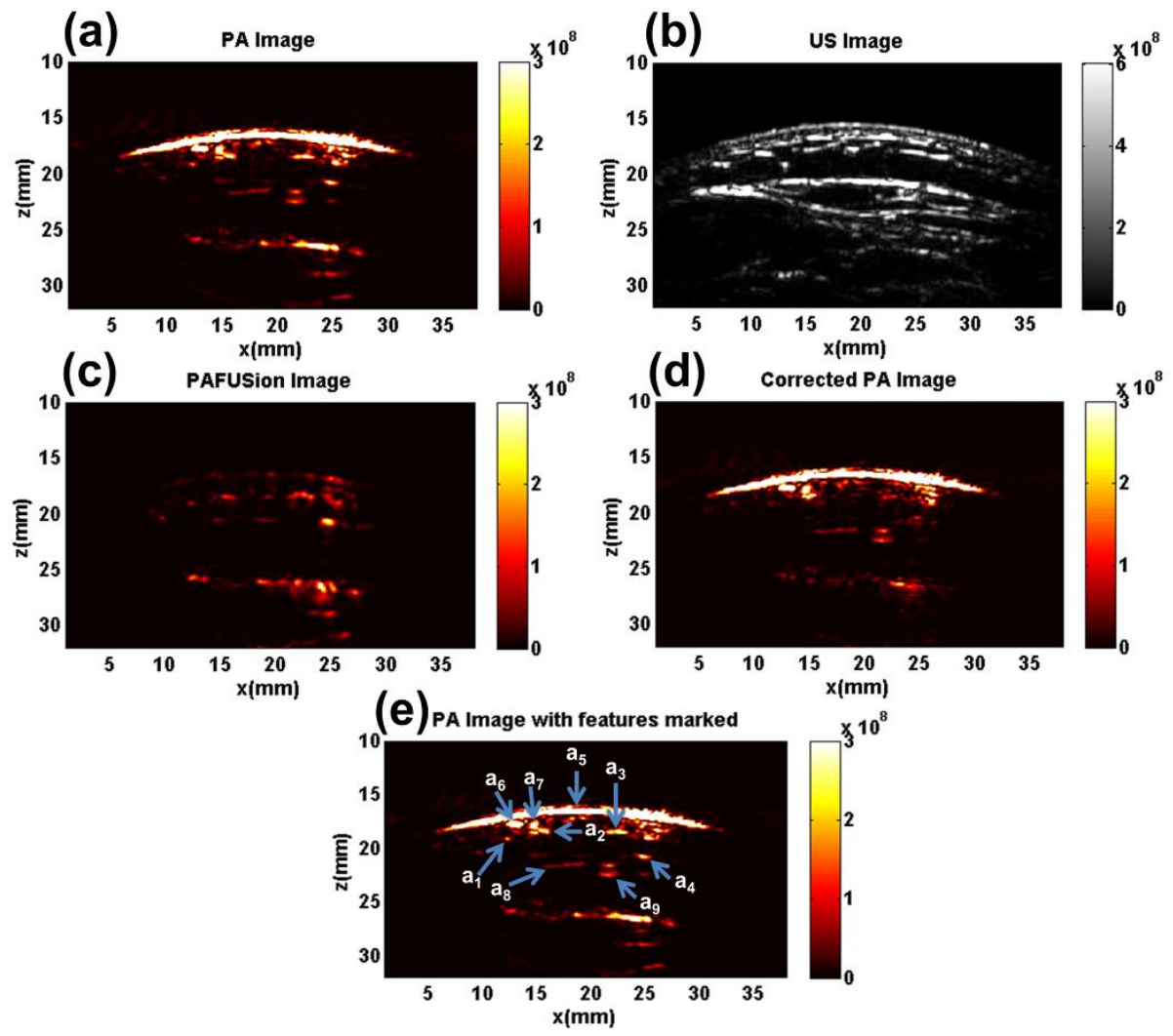

Figure 4.7 In vivo measurement 2: (a) PA image, (b) US image, (c) PAFUSion image with identified reflection artifacts, (d) corrected PA image, (e) PA image with features marked for reference.

The PAFUSion image (Figure 4.7c) shows the identified reflection artifacts. Again, in this measurement, our method shows exceptional potential in exposing that more than $60 \%$ of the bright features in the PA image of the forearm were reflection artifacts. Except for some structures ( $a_{5}$ to $a_{9}$ ), all other features ( $a_{1}$ to $a_{4}$ ) are identified as reflection artifacts, including other features that were not marked in the PA image (Figure 4.7e). All the identified artifacts are in close proximity to real PA features, confounding the image interpretation. A reflection artifact is identified at the depth of the skin-melanin layer (line like feature at around $16.5 \mathrm{~mm}$ depth), as in the previous results. Owing to the optical and acoustic contrast of the skin, this may be a real reflection artifact or an artifact of the technique as hypothesised in previous results. Figure $4.7 \mathrm{~d}$ shows the corrected PA image obtained by subtracting 
identified reflections (PAFUSion image) from the PA image. All the identified reflection artifacts are significantly reduced in the corrected PA image. The bright features left in the corrected PA image are $\mathrm{a}_{5}$ to $\mathrm{a}_{9}$. In this measurement also, PAFUSion identified several puzzling reflection artifacts below the skin surface and in the location where small blood vessels are expected. One of the examples is the bright feature $\mathrm{a}_{2}$ in the PA image (Figure 4.7e). The intensity of this feature is strongly reduced in the corrected PA image (Figure 4.7d), which aided in a better interpretation of the double-layered structure a7, which might well be a blood vessel. Another example is the feature $\mathrm{a}_{4}$ marked in the PA image (Figure 4.7e). This structure along with another less bright feature below it could be misinterpreted as a big blood vessel. After applying PAFUSion, it is clear that this feature is a reflection artifact as it is substantially reduced in the corrected PA image (Figure 4.7d). Another important feature to be discussed is as, which very well might be misinterpreted as a reflection artifact based on its extended shape. After applying our method, it is clear that $a_{8}$ is a real PA feature (possibly from a small vessel running along the muscle perimysium).

All the reflection artifacts were accurately identified in the PAFUSion image (Figure $4.7 \mathrm{c})$, maintaining the shape and the spatial details. Intensity reduction ratios were calculated for all the marked regions in the PA image. Table 4.2 summarizes these ratios for all the selected regions in the PA image for this measurement (in vivo measurement 2). It is clear from Table 4.2 that the intensity of features $a_{1}$ to $a_{4}$ (reflection artifacts) are significantly reduced in the corrected PA image, with an average reduction of around $13 \mathrm{~dB}$. For real PA features $a_{5}$ to $a_{9}$, the intensity were just reduced by less than $0.3 \mathrm{~dB}$ which is significantly less compared to the intensity reduction of artifacts.

\begin{tabular}{|c|c|c|c|c|c|c|c|c|c|}
\hline $\begin{array}{c}\text { Region } \\
\text { of } \\
\text { interest }\end{array}$ & $\mathbf{a}_{1}$ & $\mathbf{a}_{2}$ & $\mathbf{a}_{3}$ & $\mathbf{a}_{4}$ & $\mathbf{a}_{5}$ & $\mathbf{a}_{6}$ & $\mathbf{a}_{7}$ & $\mathbf{a}_{8}$ & $\mathbf{a}_{9}$ \\
\hline $\begin{array}{c}\text { IRR } \\
(\mathbf{d B})\end{array}$ & 18 & 9 & 9.5 & 13 & 0.4 & 0.2 & 0.3 & 0.1 & 0.1 \\
\hline
\end{tabular}

Table 4.2 Intensity reduction ratio (IRR) of all the marked regions in the PA image for in vivo measurement 2. 


\subsection{Discussion}

The phantom as well as the in vivo results demonstrated that PAFUSion using synthetic backpropagation is capable of identifying and strongly reducing reflection artifacts in epimode PA imaging. Using this technique, the reflection artifact intensity was reduced by around $13 \mathrm{~dB}$ on average in both phantom and in vivo measurements. The aim of this study was to give proof-of-principle of in vivo artifact reduction using synthetic backpropagation. However, PAFUSion might also be suitable for a quantitative investigation of reflection artifact occurrence (e.g. relative intensity compared to direct PA signals, as function of depth). First conclusions can be drawn from the presented in vivo results. In measurement 1 , the features $a_{1}$ to $a_{6}$ that were identified as reflection artifacts had intensity levels that were between $14 \mathrm{~dB}$ and $17 \mathrm{~dB}$ lower than the intensity of the melanin signal from which these reflections originated. Features $\mathrm{a}_{7}$ and $\mathrm{a}_{8}$ were also identified as reflections, but only $9 \mathrm{~dB}$ below the melanin signal. Similar to measurement 1 , the reflection artifacts $a_{1}$ to $a_{4}$ in measurement 2 were between $10 \mathrm{~dB}$ and $13 \mathrm{~dB}$ below the melanin signal level. In comparison, the blood vessel signal (a $\left.a_{9}\right)$ was $13 \mathrm{~dB}$ below the melanin signal level. This demonstrates that the intensity of reflection artifacts gets comparable to true PA signal intensity already at a depth of only $5 \mathrm{~mm}$ below the skin surface.

For the first time, we demonstrate identification and strong reduction of a large number of reflection artifacts just beneath the skin and in proximity to superficial vasculature. In a conventional image, these artifacts may well be misinterpreted as small blood vessels, thus demonstrating the importance of PAFUSion in identifying and reducing them. Previously reported PA clutter reduction techniques are good at reducing artifacts in deeper tissue for improving imaging depth [24, 26], but are not suited for improving contrast in shallow tissue. Removal of reflection artifacts in the region directly below the skin by using PAFUSion can help to avoid false interpretation of these features as blood vessels, inflammations, and/or a cancerous tissue. In a clinical scenario, the PAFUSion image itself will already be relevant for identifying true features in the PA image. However, reduction of reflection artifacts in the PA image via subtracting the PAFUSion has the added benefit of uncovering true PA features that are else hidden by reflection artifacts.

PAFUSion is specifically designed for reducing the echoes of the PA transients that are generated in the measurement plane. Only such echoes can be mimicked via backpropagation 
from the probe aperture, whereas the out-of-plane echoes, particularly strong when irradiating outside the probe cannot be reduced. In-plane irradiation is required for maximizing SNR of direct PA signals when using integrated systems with low diode laser pulse energies, but at the same time it limits contrast owing to the resulting echoes. PAFUSion is ideally suited for such systems, for reducing the superficial echoes within the limited imaging depth that results from the low SNR.

A main advantage of PAFUSion is that it can be implemented in any dual-mode PA/US system that provides sufficient acquisition control. Compared to LOVIT [24], it does not require US powers close to the safety limit. Compared to DCA [26], it can be used without extensive training. It is not restricted to a certain frequency band, whereas LOVIT and DCA work efficiently for the upper frequency band where a significant displacement can be achieved in comparison to the acoustic wavelength. The synthetic implementation of PA backpropagation based on reference pulse-echo data is completely software based, which makes implementation of this method in a clinical system straightforward. In this work, the backpropagation algorithm was implemented in Matlab. This was the bottleneck for processing speed and resulted in a rather slow overall framerate of around one image per three seconds. However, the display rate will be significantly improved by implementing this method on GPU (Graphical processing units). In the proposed version of PAFUSion, the reference pulse-echo data was based on single element transmissions. A different option is to use plane-wave transmissions with multiple angles. In plane-wave transmissions, the array elements are excited in a sequence. For large angles, this has the disadvantage of a long dead time during which element cross-talk can occur from transmitting elements resulting in crosstalk artifacts that obscure echoes from close to the transducer. However, this may not have a significant influence, if a gel spacer between the probe and the skin adds a sufficient delay to the arrival of first PA signals. An advantage of a plane-wave transmission approach is that it is conceptually more close to a frequency-domain formulation (with decomposition into plane- wave components) than the element-wise approach. Schwab et al [29] utilized a comparable model-based approach in which multi-angled plane-wave US transmissions were used to imitate the reflected PA waves. They derived a mathematical relation in frequency domain that links the plane-wave US measurements and a reflection-artifact-free PA measurement to the actual PA measurement. This relation is inverted in order to attain a reflection-artifact-free PA measurement. Preliminary simulation and phantom results were 
demonstrated. Such an inverse-problem approach may help avoid false reflections in the PAFUSion image, and require less US acquisitions by making use of data redundancies.

At this point, we would like to draw the attention to a limitation of the proposed technique: PA backpropagation is limited to the part of the wavefield that can be simulated by the transducer. In case of a linear array probe with limited aperture and limited transmit angle range, only transients that propagate parallel to the imaging plane and within that angle range and aperture can be reproduced by US transmission. As already predicted in the theory section, the experimental results demonstrate that this limitation is not crucial. The most important PA source - the melanin layer - could easily be aligned perpendicular to the imaging plane, aided by the quasi real-time feedback of the image display. In addition, the PA transient generated by the melanin layer contains only directional components running axially within the angular transmit aperture of the probe. The laterally propagating transients generated by the superficial blood vessels, on the other hand, do not seem to have produced significant reflection artifacts.

Another important point to consider is the role of US attenuation in the applicability of the technique. When backpropagating the PA signal to the origin, it has undergone propagation through the tissue twice, resulting in additional attenuation compared to the real PA transients that generate the reflection artifacts. Therefore, it is expected that the synthetically mimicked echoes do not perfectly match the real ones. This apparently did not play much of a role in our experiments, owing to the shallow imaging depth. For applications requiring larger imaging depth, this problem is expected to become prominent. We foresee to solve this by a depth-dependent impulse response matching, based on an assumed attenuation map of the tissue.

All clinical applications of epi-mode PA imaging could benefit from the efficient reflection artifact correction achieved using our novel method. One of the important sources of PA reflection artifacts is the skin melanin layer. Thus, PAFUSion will help to avoid the exclusion of dark skinned patients from PA clinical measurements/clinical trials. The impact of PAFUSion will also be high for imaging structures such as finger joints or wrists where multiple small blood vessels and highly reflecting tendons and bones are present in a dense space. Furthermore, reflections of high PA transients generated at the tip of the optical fiber is a well-known problem in PA guidance of minimally invasive procedures [33-35]. These reflection artifacts can be potentially identified and reduced using the proposed technique. 
The present study focused on reflection artifact reduction in epi-mode PA imaging using handheld probes. Reflection artifacts generated by PA transients from the skin surface are most disturbing in epi-PA imaging because they overlap in time with the weak PA signals from inside the tissue where the fluence is comparably low. In comparison, tomographic systems with curved arrays in two or three dimensions typically use a transmission- or orthogonal-mode irradiation geometry, where a better distinction may be possible because the fluence inside the tissue is relatively higher. However, strongly reflecting structures such as air-filled cavities or bones can lead to prominent reflections in PA tomography of small animals. It has been shown that significant artifact reduction is possible by using a weighted back-projection approach, where image reconstruction is selectively based on PA signals that are least disturbed by reflectors [30]. We hypothesize that PAFUSion will even be helpful in such systems because it allows artifact reduction without data reduction, potentially providing improved spatial resolution of real PA sources.

\subsection{Conclusions}

In summary, PAFUSion can identify and strongly reduce in-plane reflection artifacts, and thus envisions potential in improving PA imaging of acoustically inhomogeneous tissue. In both phantom and in vivo measurements, the intensity of reflection artifacts was on average reduced by around $13 \mathrm{~dB}$, which is certainly a promising result. For the first time, we demonstrated the relevance of identifying and reducing superficial PA reflection artifacts to improve image interpretation. PAFUSion will thus be a useful addition to any epi-mode $\mathrm{PA} / \mathrm{US}$ system. 


\subsection{References}

1. P. Beard, Biomedical photoacoustic imaging. Interface Focus, 2011. 1(4): p. 60231.

2. M. Xu and L.V. Wang, Photoacoustic imaging in biomedicine. Review of Scientific Instruments, 2006. 77.

3. S. $\mathrm{Hu}$ and L.V. Wang, Photoacoustic imaging and characterization of the microvasculature. Journal of Biomedical Optics, 2010. 15(1).

4. H.F. Zhang, et al., Functional photoacoustic microscopy for high-resolution and noninvasive in vivo imaging. Nat Biotechnol, 2006. 24(7): p. 848-51.

5. X.L. Dean-Ben and D. Razansky, Adding fifth dimension to optoacoustic imaging: volumetric time-resolved spectrally enriched tomography. Light-Science \& Applications, 2014. 3.

6. X.L. Dean-Ben, E. Bay, and D. Razansky, Functional optoacoustic imaging of moving objects using microsecond-delay acquisition of multispectral threedimensional tomographic data. Scientific Reports, 2014. 4.

7. A. Hussain, et al., Quantitative blood oxygen saturation imaging using combined photoacoustics and acousto-optics. Optics Letters, 2016. 41(8): p. 1720-1723.

8. S.A. Ermilov, et al., Laser optoacoustic imaging system for detection of breast cancer. Journal of Biomedical Optics, 2009. 14(2).

9. M. Heijblom, et al., Photoacoustic image patterns of breast carcinoma and comparisons with Magnetic Resonance Imaging and vascular stained histopathology. Scientific Reports, 2015. 5.

10. P. van Es, et al., Initial results of finger imaging using photoacoustic computed tomography. J Biomed Opt, 2014. 19(6): p. 60501.

11. X. Wang, et al., Photoacoustic imaging of human inflammatory arthritis. 2015 Ieee International Ultrasonics Symposium (Ius), 2015.

12. L. Xi and H.B. Jiang, High resolution three-dimensional photoacoustic imaging of human finger joints in vivo. Applied Physics Letters, 2015. 107(6).

13. D.R. Bauer, et al., 3-D photoacoustic and pulse echo imaging of prostate tumor progression in the mouse window chamber. Journal of Biomedical Optics, 2011. 16(2).

14. C.L. Bayer, et al., Multiplex photoacoustic molecular imaging using targeted silicacoated gold nanorods. Biomedical Optics Express, 2011. 2(7): p. 1828-1835.

15. M. Kuniyil Ajith Singh, W. Steenbergen, and S. Manohar, Handheld Probe-Based Dual Mode Ultrasound/Photoacoustics for Biomedical Imaging, in Frontiers in Biophotonics for Translational Medicine, M. Olivo and U.S. Dinish, Editors. 2016, Springer Singapore. p. 209-247.

16. J.J. Niederhauser, et al., Combined ultrasound and optoacoustic system for realtime high-contrast vascular imaging in vivo. Ieee Transactions on Medical Imaging, 2005. 24(4): p. 436-440.

17. R.G.M. Kolkman, et al., Real-time in vivo photoacoustic and ultrasound imaging. Journal of Biomedical Optics, 2008. 13(5).

18. A. Aguirre, et al., Coregistered three-dimensional ultrasound and photoacoustic imaging system for ovarian tissue characterization. Journal of Biomedical Optics, 2009. 14(5). 
19. M. Jaeger, et al., Reduction of background in optoacoustic image sequences obtained under tissue deformation. Journal of Biomedical Optics, 2009. 14(5).

20. K. Daoudi, et al., Handheld probe integrating laser diode and ultrasound transducer array for ultrasound/photoacoustic dual modality imaging. Optics Express, 2014. 22(21): p. 26365-26374.

21. C. Kim, et al., Deeply penetrating in vivo photoacoustic imaging using a clinical ultrasound array system. Biomedical Optics Express, 2010. 1(1): p. 278-284.

22. G. Xu, et al., Photoacoustic and ultrasound dual-modality imaging of human peripheral joints. Journal of Biomedical Optics, 2013. 18(1).

23. A. Needles, et al., Development and Initial Application of a Fully Integrated Photoacoustic Micro-Ultrasound System. Ieee Transactions on Ultrasonics Ferroelectrics and Frequency Control, 2013. 60(5): p. 888-897.

24. M. Jaeger, J.C. Bamber, and M. Frenz, Clutter elimination for deep clinical optoacoustic imaging using localised vibration tagging (LOVIT). Photoacoustics, 2013. 1(2): p. 19-29.

25. M. Kuniyil Ajith Singh and W. Steenbergen, Photoacoustic-guided focused ultrasound (PAFUSion) for identifying reflection artifacts in photoacoustic imaging. Photoacoustics, 2015. 3(4): p. 123-131.

26. M. Jaeger, et al., Deformation-compensated averaging for clutter reduction in epiphotoacoustic imaging in vivo. Journal of Biomedical Optics, 2012. 17(6).

27. G. Held, et al., Effect of irradiation distance on image contrast in epi-optoacoustic imaging of human volunteers. Biomedical Optics Express, 2014. 5(11): p. 37653780 .

28. E.J. Alles, M. Jaeger, and J.C. Bamber, Photoacoustic Clutter Reduction Using Short-Lag Spatial Coherence Weighted Imaging. 2014 Ieee International Ultrasonics Symposium (Ius), 2014: p. 41-44.

29. H.M. Schwab, M.F. Beckmann, and G. Schmitz, Photoacoustic clutter reduction by inversion of a linear scatter model using plane wave ultrasound measurements. Biomedical Optics Express, 2016. 7(4): p. 1468-1478.

30. X.L. Dean-Ben, V. Ntziachristos, and D. Razansky, Artefact reduction in optoacoustic tomographic imaging by estimating the distribution of acoustic scatterers. Journal of Biomedical Optics, 2012. 17(11).

31. T.F. Fehm, X.L. Dean-Ben, and D. Razansky, Four dimensional hybrid ultrasound and optoacoustic imaging via passive element optical excitation in a hand-held probe. Applied Physics Letters, 2014. 105(17).

32. M. Jaeger, et al., Fourier reconstruction in optoacoustic imaging using truncated regularized inverse k-space interpolation. Inverse Problems, 2007. 23(6): p. S51S63.

33. D. Piras, et al., Photoacoustic needle: minimally invasive guidance to biopsy. J Biomed Opt, 2013. 18(7): p. 070502.

34. W. Xia, et al., Performance characteristics of an interventional multispectral photoacoustic imaging system for guiding minimally invasive procedures. J Biomed Opt, 2015. 20(8): p. 86005.

35. M.A.L. Bell, et al., In vivo visualization of prostate brachytherapy seeds with photoacoustic imaging. Journal of Biomedical Optics, 2014. 19(12). 


\title{
Photoacoustic reflection
}

\section{artifact reduction using}

\section{photoacoustic-guided focused}

\section{ultrasound: Comparison between}

\author{
plane-wave and element-by- \\ element synthetic
}

\section{backpropagation approach $^{4}$}

Reflection artifacts caused by acoustic inhomogeneities constitute a major problem in epimode biomedical photoacoustic imaging. Photoacoustic transients from the skin and superficial optical absorbers traverse into the tissue and reflect off echogenic structures to generate reflection artifacts. These artifacts cause difficulties in the interpretation of images and reduce contrast and imaging depth. We recently developed a method called PAFUSion (photoacoustic-guided focused ultrasound) to circumvent the problem of reflection artifacts in photoacoustic imaging. We already demonstrated that the photoacoustic signals can be backpropagated using synthetic aperture pulse-echo data for identifying and reducing

4 This chapter is being prepared for publication as: M. Kuniyil Ajith Singh, M. Jaeger, M. Frenz, and W. Steenbergen; "Photoacoustic reflection artifact reduction using photoacoustic-guided focused ultrasound: Comparison between plane-wave and element-by-element synthetic backpropagation approach". 
reflection artifacts in vivo. In this work, we propose an alternative variant of PAFUSion in which synthetic backpropagation of photoacoustic signals is based on multi-angled planewave ultrasound measurements. We implemented plane-wave and synthetic aperture PAFUSion in a handheld ultrasound/photoacoustic imaging system and demonstrated reduction of reflection artifacts in phantoms and in vivo measurements on a human finger using both approaches. Our results suggest that, while both approaches are equivalent in terms of artifact reduction efficiency, plane-wave PAFUSion requires less pulse-echo acquisitions when the skin absorption is the main cause of reflection artifacts. 


\subsection{Introduction}

Photoacoustic (PA) imaging (optoacoustic imaging) allows the detection of optical absorption contrast inside optically scattering tissue via ultrasound (US) detection of thermoelastically induced US, when irradiating the tissue with pulsed laser light $[1,2]$. This technique offers great promise in a wide range of pre-clinical and clinical applications [3-7].

Since PA imaging involves detection of US signals, integration with conventional pulseecho US in a single multi-modal device is a logical development. A PA image superimposed on a US image can give anatomical and structural information overlaid with complementary functional information of e.g. the local blood oxygenation level [8]. Handheld dual mode US/PA probes use an epi-illumination geometry, where the optical components are integrated with the US probe to irradiate the tissue from the same side where PA signals are detected [9]. Epi-PA imaging provides freehand probe guidance and large flexibility in imaging different body parts [10]. On the downside, high light fluence beneath the US probe results in strong PA signals from the skin and superficial blood vessels. These PA transients will traverse into the tissue and reflect off the echogenic structures to generate reflection artifacts (echo clutter) [10-13], that can significantly reduce contrast and imaging depth [10, 13]. In addition, reflection artifacts just beneath the skin and in close proximity to blood vessels may confuse diagnosis by being misinterpreted as additional blood vessels or by biasing multiwavelength imaging results. Thus, it is important to identify and reduce in-plane reflection artifacts for clinically successful high-contrast deep tissue epi-PA imaging.

Several approaches for identifying and reducing reflection artifacts have been reported $[10,11,14-16]$. We recently reported on our first in vivo results of synthetic aperture PAFUSion [13]. In this technique, PA signals are virtually backpropagated using synthetic aperture US data, for imitating the inward-travelling PA field and thus the resulting reflection artifacts. In this work, we propose a modification of this technique where synthetic backpropagation is based on multi-angled plane-wave transmissions as opposed to elementby-element transmission of US. The goal of this paper is a quantitative comparison of reflection artifact reduction achieved using plane-wave and synthetic aperture PAFUSion implemented in a handheld US/PA imaging system. We demonstrate strong in-plane artifact reduction using both methods in a phantom as well as in vivo measurements on a finger of a 
human volunteer. Both approaches are systematically compared to conclude on the most suitable implementation in a clinical scenario.

\subsection{Theory}

In general, PAFUSion mimics the PA wavefield traversing into the tissue and the resulting reflection artifacts by using US transmissions [12, 13]. In the optimum case, this shall allow to subtract the identified artifacts from the PA signal and thus obtain a reflection-artifact-free PA image. In the less optimum case, this shall allow the identification of reflection artifacts as such, and thus a more accurate interpretation of "true" PA features.

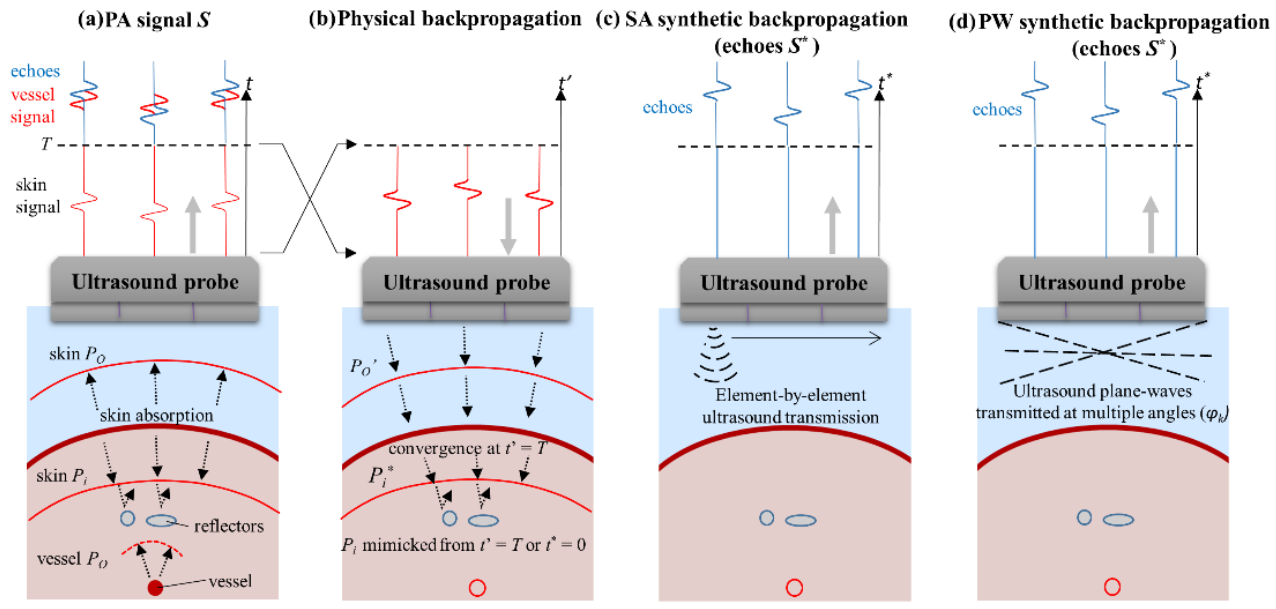

Figure 5.1 (a) Illustration of inward ( $P_{i}$ propagating into the tissue) and outwardpropagating ( $P_{O}$ propagating towards the probe) PA transients, generated by optical absorption in the skin melanin layer. (b) Time-inversion and backpropagation of the PA signal from interval $t=[0, T]$ towards mimicking the PA wavefield $P_{\mathrm{i}}^{*}$ traversing into the tissue and identification of resulting echoes, (c) Element-by-element US transmissions (SA) for synthetically backpropagating outward propagating skin PA signal $\left(P_{O}\right)$ and thus to mimic inward propagating skin PA signal $\left(P_{i}\right),(\mathrm{d})$ Transmission of plane-waves $(\mathrm{PW})$ with multiple angles for synthetically backpropagating outward propagating skin PA signal $\left(P_{O}\right)$ and thus to mimic inward propagating skin PA signal $\left(P_{i}\right)$. 
PAFUSion using backpropagation [13] is based on the concept that the PA wave field can be decomposed to an outward-travelling part $\left(P_{O}\right)$ that is detected as "true" PA signal by the transducer, and an inward-travelling part $\left(P_{i}\right)$ that reflects off the echogenic structures to generate reflection artifacts (Figure 5.1a). At time $t=0$, the two wave fields are identical but move in opposite directions. Thus, backpropagation of the wave field that was detected within the time interval $[0, T]$ reproduces after time $T$ the inward travelling wave field (Figure 5.1b). A possible value of $T$ is shown in Figure 5.1a. The US echoes obtained after backpropagation will be similar to the reflection artifacts in the PA image. Physical backpropagation of PA signals requires the capability of transmitting arbitrarily shaped signals, which is beyond state-of-the-art of US equipment. In our previous work, we demonstrated the potential of using element-by-element US data to synthetically backpropagate PA signals and thus identify and reduce reflection artifacts [13]. Synthetic aperture PAFUSion involves convolution of the time-inverted PA signal from each element with the Dirac delta tissue response (defined as the echoes detected after transmission on a respective element), and subsequent superposition of the results for all elements. The resulting synthetized data mimic the PA reflection artifacts and are used to correct the PA images to obtain reflection-artifact-free PA images. Figure 5.1c shows synthetic backpropagation of skin PA signal using synthetic aperture transmission of US. In this case, it is obvious that a scan through the whole aperture, with one element transmitting at a time, is required to mimic the inward travelling PA wave field from skin layer.

While the synthetic aperture approach is conceptually simple, other ways of generating reference pulse-echo data are possible and may be more efficient depending on the spatial distribution of artifact-generating PA sources. In the case where skin absorption is the main cause of reflection artifacts, the strong PA transient generated by the skin is close to a plane transient, and therefore a superposition of a sparse number of plane transients may be sufficient to mimic most part of the PA wavefield. This hypothesis inspired the implementation of an alternative approach, where virtual backpropagation is based on US plane-wave acquisitions with different transmit steering angles instead of the element-byelement US transmissions (Figure 5.1d). In analogy to the description of synthetic backpropagation algorithm in our previous paper [13], step by step explanation of planewave PAFUSion is as follows: 
1. Acquire reference plane wave pulse-echo data, $S_{\delta_{k}}\left(n^{*}, t^{\prime \prime}\right)$ detected on elements $n^{*}$ after transmission with transmission angles $\varphi_{k}$

2. Filter PA signals $S(n, t)$ for directions $\varphi_{k}$ by time-delaying $S(n, t)$ for each element by delay $\tau\left(\varphi_{\mathrm{k}}, n\right)$ that corresponds to the arrival time of a plane wave under angle $\varphi_{k}$ at elements $n$. Average over $n$, resulting in a filtered signal $\hat{S}\left(\varphi_{k}\right.$ ,t)

3. Convolve $\hat{S}\left(\varphi_{k}, T-t^{\prime}\right)$ with $S_{\delta k}\left(n^{*}, t^{\prime \prime}\right)$ to obtain Signals $S^{*}\left(n^{*}, \varphi_{k}, t^{\prime \prime}\right)$

4. Superpose $S^{*}\left(n^{*}, \varphi_{k}, t^{\prime \prime}\right)$ obtained for different transmission angles and shift in time according to $t^{*}=t^{\prime \prime}-T$ to obtain $\mathrm{S}^{*}\left(n^{*}, t^{*}\right)$

$S^{*}$ represents the image containing only the mimicked reflection artifacts.

\subsection{Materials and methods}

\subsubsection{Equipment and setup}

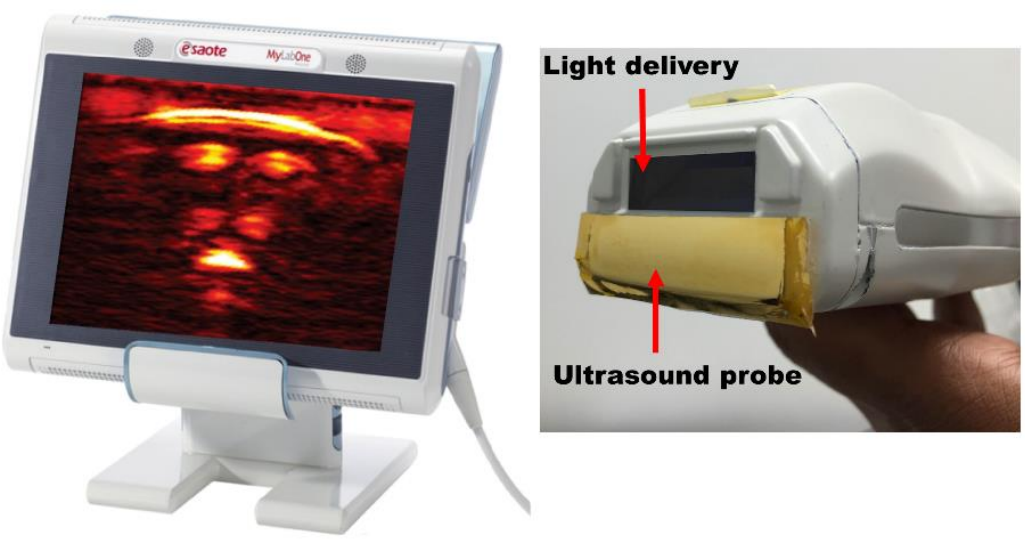

Figure 5.2 Photograph of the commercial US system showing a real-time reconstructed PA image of a human finger (left) and the hybrid probe (right) integrating the laser module and the US transducer array (US probe is optically shielded with gold foil).

For all the experiments in this study, a handheld probe-based dual mode PA/US system was used [17]. Figure 5.2 shows the photograph of the system in which a commercial US scanner (MyLabOne, Esaote Europe BV, The Netherlands) is used along with a probe that integrates a US array with a diode laser module. The module produced pulses at $805 \mathrm{~nm}$ wavelength, $130 \mathrm{~ns}$ pulse width at an energy of $1 \mathrm{~mJ}$ per pulse, with a high pulse repetition frequency of up to $10 \mathrm{kHz}$. The US probe has a $-6 \mathrm{~dB}$ fractional bandwidth of around $100 \%$ and center 
frequency of $7.5 \mathrm{MHz}$. For avoiding optical absorption in the acoustic lens surface, the aperture was optically shielded with a $100 \mu \mathrm{m}$ thin gold foil.

The US scanner was used in research mode that provided access to all transmission and acquisition parameters. Custom-made software running on a laptop (connected to the scanner using USB 2.0 connection) controlled the US scanner for triggering laser diodes and US transmission. The same software also communicated with the scanner for sequential switching between PA, synthetic aperture US, and multi-angled plane-wave US measurements. The number of elements to transmit for synthetic aperture US and the number of angles for plane-wave US measurements were also controlled by the scanner. The system can read data from 64 channels at a time with a sampling rate of $50 \mathrm{MHz}$, and perform continuous data transfer for real-time processing running on the laptop. For both the phantom and in vivo studies, 64 middle elements of the aperture were used for synthetic aperture US (one element transmits at a time resulting in 64 acquisitions) and angles of $-16^{\circ}$ to $+16^{\circ}$ with a step of $1^{\circ}$ for plane-wave US measurements (resulting in 33 acquisitions). 200 frames of PA data were also saved for further processing. PA, plane-wave and synthetic aperture US measurements were performed with a repetition rate of $200 \mathrm{~Hz}$ resulting in an acquisition time of less than 2 seconds (acquisition of PA, synthetic aperture US and plane-wave US data). All the post-processing presented was performed offline, including image reconstruction using a frequency domain algorithm [18].

\subsubsection{Plane-wave and synthetic aperture PAFUSion - Data processing}

The processing steps for synthetic aperture PAFUSion are the same as described in our previous work [13]. Plane-wave PAFUSion was performed following the steps described in the Theory section. Echoes obtained by transmission of multi-angled plane-waves were used as the reference for synthetic backpropagation. PA signals were filtered for different directions corresponding to the angles used for US transmission and convolved with the pulse-echo data for that particular angle transmission (i.e. for each transmission angle, there will be a corresponding PA data obtained by time-delaying for each element by the time that corresponds to the arrival time of a plane-wave under that particular angle). Superposition of convolution results for all the angles generated a PAFUSion image with only the identified reflection artifacts. PAFUSion image $\left(S^{*}\right)$ was subtracted from the PA image $(S)$ to generate corrected PA image without reflection artifacts. To match the waveforms of the synthetically 
generated and the real reflection artifacts - and thus enable accurate subtraction $-S$ and $S^{*}$ were convolved with a matched pair of finite impulse response (FIR) filters, one for each type of signal before the subtraction step. These filters were calibrated prior to the experiments in a simple setup with a plane steel reflector as described in our previous study [13]. Also, the amplitudes and phases of the identified artifacts were matched to their counterparts in the PA image before subtraction for efficient correction of reflection artifacts. For the phantom measurement, the amplitude and phase matching were performed for every identified artifact separately to generate multiple corrected PA images (each with one artifact eliminated). For the in vivo measurement, amplitude and phase matching were performed for only one of the identified artifacts (one step subtraction by matching the amplitude and phase of one identified artifact with its counterpart in the PA image) for ease of interpretation and to avoid numerous corrected PA images.

\subsubsection{Phantom experiment}

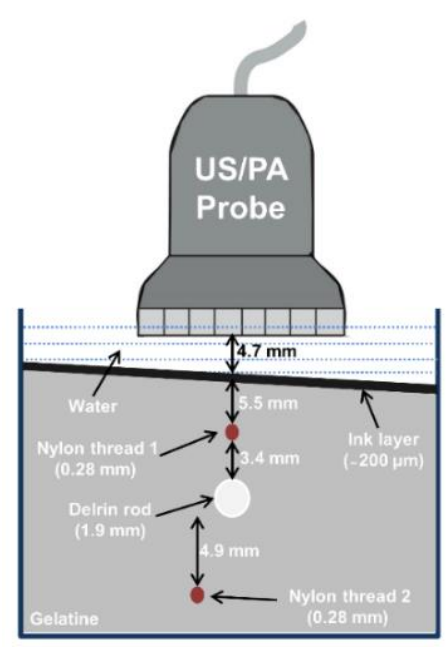

Figure 5.3 Schematic of the phantom and orientation of the US/PA probe.

Figure 5.3 shows the schematic of the phantom and the orientation of the US/PA probe used for the comparison of plane-wave and synthetic aperture PAFUSion. The phantom consisted of two optical absorbers (brown nylon threads, $0.28 \mathrm{~mm}$ diameter) positioned above and below an acoustic reflector (Delrin rod, $1.9 \mathrm{~mm}$ diameter). Both the optical absorbers and acoustic reflector were embedded inside gelatin, in such a way that they were perpendicular 
to the imaging plane of the US transducer. A thin layer of black ink (India ink, $\sim 200 \mu \mathrm{m}$ ) was added to the surface of the gelatin phantom to mimic the optical absorption by the skin melanin layer and thus generating reflection artifacts. A water layer in-between the phantom surface and the US probe was used for acoustic coupling. This phantom was used to study reflection artifacts caused by both the extended PA features (eg: skin) and vessel-like features, and to compare the performances of plane-wave and synthetic aperture PAFUSion with these features. No optical scattering agent was added to the bulk of the gelatin phantom, to maximize the amplitude of the PA transients (and thereby of the PA reflections). In this experiment, the time $T$ ( $8 \mu \mathrm{s}$, time until which only real PA signals are expected) was selected in such a way, so as to backpropagate the PA signal from the skin-mimicking surface and the first nylon thread.

\subsubsection{In vivo experiment - Human finger}

Evaluation of plane-wave and synthetic aperture PAFUSion for the reduction of reflection artifacts in vivo was performed by measurements on the index finger of a human volunteer with dusky skin complexion. The finger was positioned inside a water bath in such a way that the imaging plane was aligned perpendicular to the finger (between the distal interphalangeal joint and proximal interphalangeal joint in the sagittal part). Real-time PA reconstruction provided a feedback to select the location for the measurements. For in vivo measurements, $T$ was selected in such a way that the PA signals from only the skin and the superficial blood vessels were synthetically backpropagated for performing PAFUSion.

\subsubsection{Quantitative comparison of plane-wave and synthetic aperture PAFUSion}

Performance of plane-wave and synthetic aperture PAFUSion were compared based on the efficiency in reducing reflection artifacts. For this purpose, pixel intensities of selected regions of interest (ROI) in the images were compared, before and after correction of PA images using plane-wave and synthetic aperture PAFUSion. The pixel intensity of a ROI was calculated as the average intensity of 9 pixels ( 3 by 3 ) centered around the maximum intensity value in the area. All features with pixel intensities higher than $1 \mathrm{~dB}$ were used for comparison and some of them are discussed in detail in the results section. Pixel intensities of the same selected regions were determined in the uncorrected $\left(\mathrm{PA}_{\mathrm{i}}\right)$ and in the corrected PA image $\left(\mathrm{CorrPA}_{\mathrm{i}}\right)$. The ratio of $\mathrm{PA}_{\mathrm{i}}$ and CorrPA $\left(\right.$ Intensity reduction ratio $=\mathrm{PA}_{\mathrm{i}} / \mathrm{CorrPA}_{\mathrm{i}}$ ) 
was calculated and expressed in $\mathrm{dB}$. In the phantom experiment where multiple corrected PA images are presented, CorrPA $\mathrm{A}_{\mathrm{i}}$ values for regions not identified as artifacts are obtained by taking average CorrPA $\mathrm{A}_{\mathrm{i}}$ values of those regions in all the corrected PA images. For the identified artifacts, CorrPA $\mathrm{A}_{\mathrm{i}}$ values are the pixel intensities of the region where artifact reduction is demonstrated in each corrected PA image. The intensity reduction ratio obtained for different ROIs using plane-wave and synthetic aperture PAFUSion are compared to conclude on the performance of both the approaches in reflection artifact reduction.

\subsection{Results}

In all images, lateral and axial coordinates are represented by $\mathrm{x}$ and $\mathrm{z}$ respectively. The envelopes of the images (PA, US, PAFUSion and corrected PA) are squared and displayed in $\mathrm{dB}$ scale.

\subsubsection{Phantom experiment}
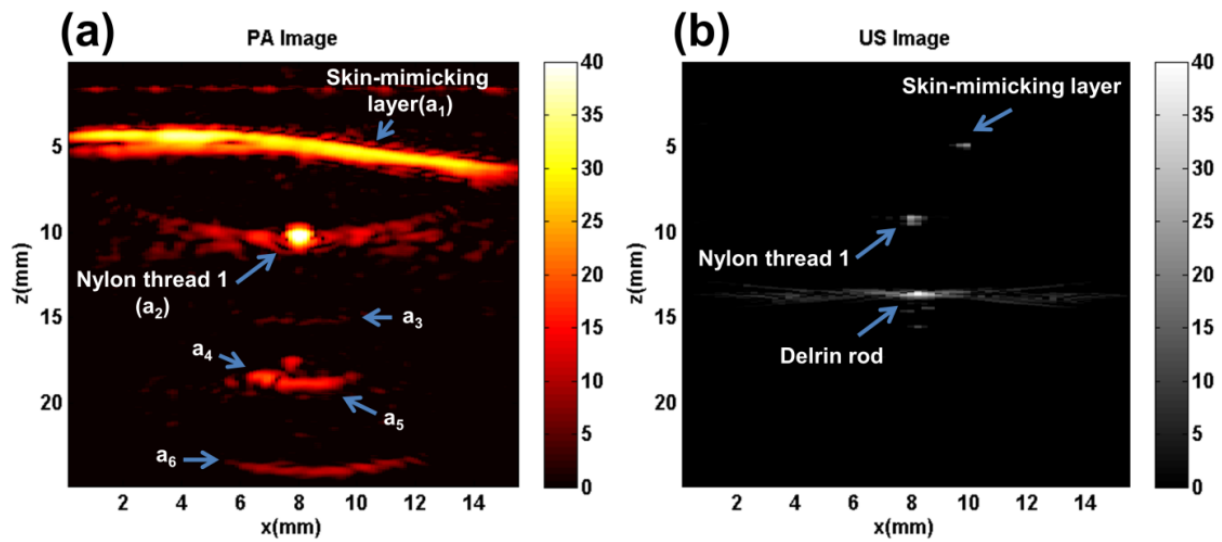

Figure 5.4 Phantom experiment results: (a) PA image with several features marked (a - a6), (b) US image.

Figure 5.3 schematically illustrates the details of the phantom and the orientation of the hybrid PA/US probe in the experimental setup. Echoes from the skin-mimicking layer, nylon thread 1 and the Delrin rod are visible in the synthetic aperture US image (Figure 5.4b). The skin-mimicking layer is seen as a point-like feature most likely because of the inclined angle of the top surface of the phantom. The second nylon thread is not visible in the US image 
because of the choice of the $\mathrm{dB}$ range ( $\mathrm{dB}$ range is chosen in such a way that image is not saturated). Figure 5.4a shows the PA image of the phantom where multiple bright features are visible. The skin-mimicking layer (marked as $\mathrm{a}_{1}$ ) and nylon thread 1 (marked as $\mathrm{a}_{2}$ ) are visible in the PA image. There is no evident point-like feature visible at the depth of nylon thread 2 (position marked as $\mathrm{a}_{4}$ ) in the PA image. Near the expected position of thread 2, there is a horizontally extended feature $\left(a_{5}\right)$ visible, which does not correspond to any optical absorber in the phantom. In addition, there are two further features $\left(\mathrm{a}_{3}\right.$ and $\left.\mathrm{a}_{6}\right)$ visible in the PA image.
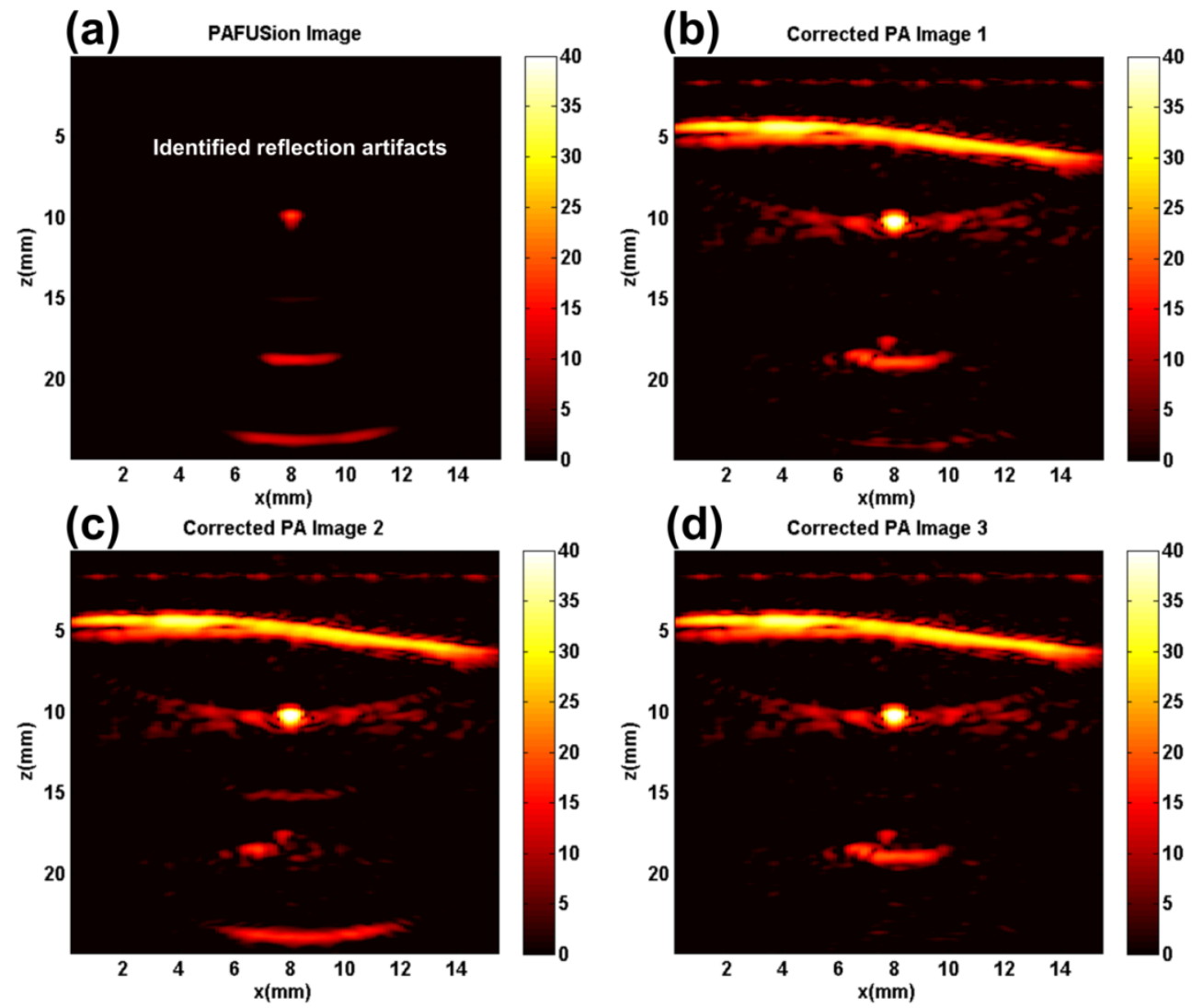

Figure 5.5 Plane-wave PAFUSion to identify and reduce reflection artifacts in the phantom experiment: (a) PAFUSion image, (b) corrected PA image in which the pixel intensities of the feature $a_{3}$ is reduced, (c) corrected PA image in which the pixel intensities of the feature as is reduced, (d) corrected PA image in which the pixel intensities of the feature a6 is reduced. 
Figure 5.5 shows the results of using plane-wave PAFUSion for identifying and reducing reflection artifacts in the phantom experiment. Figure 5.5a shows the PAFUSion image with all the identified reflection artifacts. Three of the features $\left(a_{3}, a_{5}\right.$ and $\left.a_{6}\right)$ visible in the PA image (Figure 5.4a) are identified as artifacts using plane-wave PAFUSion. Feature $\mathrm{a}_{3}$ is weakly visible in the PAFUSion image (Figure 5.5a) because of the chosen $\mathrm{dB}$ scale. Features $\mathrm{a}_{3}$ and $\mathrm{a}_{6}$ would have been caused by the reflection of the strong PA transient from the skinmimicking layer on nylon thread 1 and Delrin rod respectively. Reflection of the nylon thread 1 signal on the Delrin rod would have resulted in the feature $a_{5}$. A reflection artifact was identified at the location of nylon thread 1 also. This may be the result of reflection of PA transient from nylon thread 1 reflecting off its own surface during signal generation or an artifact of the technique caused by the result of reflections of the backpropagated wavefield at the absorber surface, immediately before it converges at $t^{*}=0$. However, the pixel intensity of this artifact is lower compared to the actual PA signal from the nylon thread and does not affect any of the image correction steps [13]. The shape and spatial details of the identified reflection artifacts match the reflection artifacts in the PA image well. The relative amplitudes and phases of the identified artifacts are not exactly equal to the artifacts in the PA image. Most likely, this is because of the unpredictable pulse-echo amplitudes in the nearfield of the US probe.

Figure 5.5b-d show the corrected PA images (each one with one artifact eliminated) obtained by subtracting the PAFUSion image from the PA image. In the first step of correction, the amplitude and phase of the reflection artifact identified at the depth of $\mathrm{a}_{3}$ was matched to its counterpart in the PA image. As a result, the pixel intensity of the feature $\mathrm{a}_{3}$ is considerably reduced in the first corrected PA image (Figure 5.5b) compared to the uncorrected one (Figure 5.4a). Along with $\mathrm{a}_{3}$, intensity of $\mathrm{a}_{6}$ is also reduced. It is worth mentioning that the pixel intensities of the features which are not identified as artifacts (actual PA features) are not visibly changed. Figure 5.5c shows the corrected PA image in which pixel intensity of the feature $a_{5}$ is significantly reduced. In this case, amplitude and phase matching were performed separately for feature $a_{5}$. It is interesting to notice that the nylon thread 2 which was obscured by an artifact in the PA image becomes evident as a point-like feature after the correction. This demonstrates the effectiveness of PAFUSion in revealing PA features that were initially mixed with reflection artifacts. In this case, even though the intensity of feature a5 could be reduced, the intensity level of feature $a_{6}$ was increased. Figure 
5.5d shows the last corrected PA image in which the amplitude and phase matching filters were adapted to reduce the intensity of feature $\mathrm{a}_{6}$. Along with $\mathrm{a}_{6}$, intensity of feature $\mathrm{a}_{3}$ is also reduced. Different steps of amplitude and phase matching were thus required for reducing intensities of different artifacts, and partially some artifacts were increased while reducing others. The reason for this is the different relative amplitude and phase of different artifacts in the PAFUSion image as compared to the real PA image.

The ratio of PA pixel intensities before and after correction was calculated for all the regions marked in the PA image (Figure 5.4a). For the corrected PA pixel intensities, separate corrected PA images are considered. For example, the corrected image value of feature $\mathrm{a}_{6}$ was calculated from Figure 5.5d, where this feature was corrected. All the reflection artifact reduction ratio values are summarized in $\mathrm{dB}$ scale in Table 5.1 to quantitatively validate the efficiency of plane-wave PAFUSion in reducing reflection artifacts.

Figure 5.6 shows the results of using synthetic aperture PAFUSion for identifying and reducing reflection artifacts in the phantom experiment. As for the plane-wave PAFUSion results, all the artifacts were accurately identified and reduced using synthetic aperture PAFUSion. Both the approaches were equally capable of uncovering reflection artifacts caused by both an extended PA source (skin-mimicking layer) and a point-like PA source (nylon thread perpendicular to the probe). It is important to mention that plane-wave PAFUSion was performed with almost half number of acquisitions (33 acquisitions, double the frame rate) compared to the synthetic aperture approach (based on the hypothesis that a smaller number of plane transients can mimic the PA wavefield from skin). Reflection artifact reduction ratio values for all the marked features in the PA image are summarized in $\mathrm{dB}$ scale in Table 5.1 to quantitatively validate and compare the efficiency of synthetic aperture PAFUSion with plane-wave PAFUSion in reducing reflection artifacts. It is clear that both approaches are similar in terms of reflection artifact reduction efficiency. Using both the approaches, the amplitudes of identified reflection artifacts $\left(\mathrm{a}_{3}, \mathrm{a}_{5}\right.$, and $\left.\mathrm{a}_{6}\right)$ are reduced on an average of $14 \mathrm{~dB}$ substantiating that these are artifacts. On the other hand, pixel intensities of real PA features $\left(\mathrm{a}_{1}, \mathrm{a}_{2}\right.$, and $\left.\mathrm{a}_{4}\right)$ are not affected. 

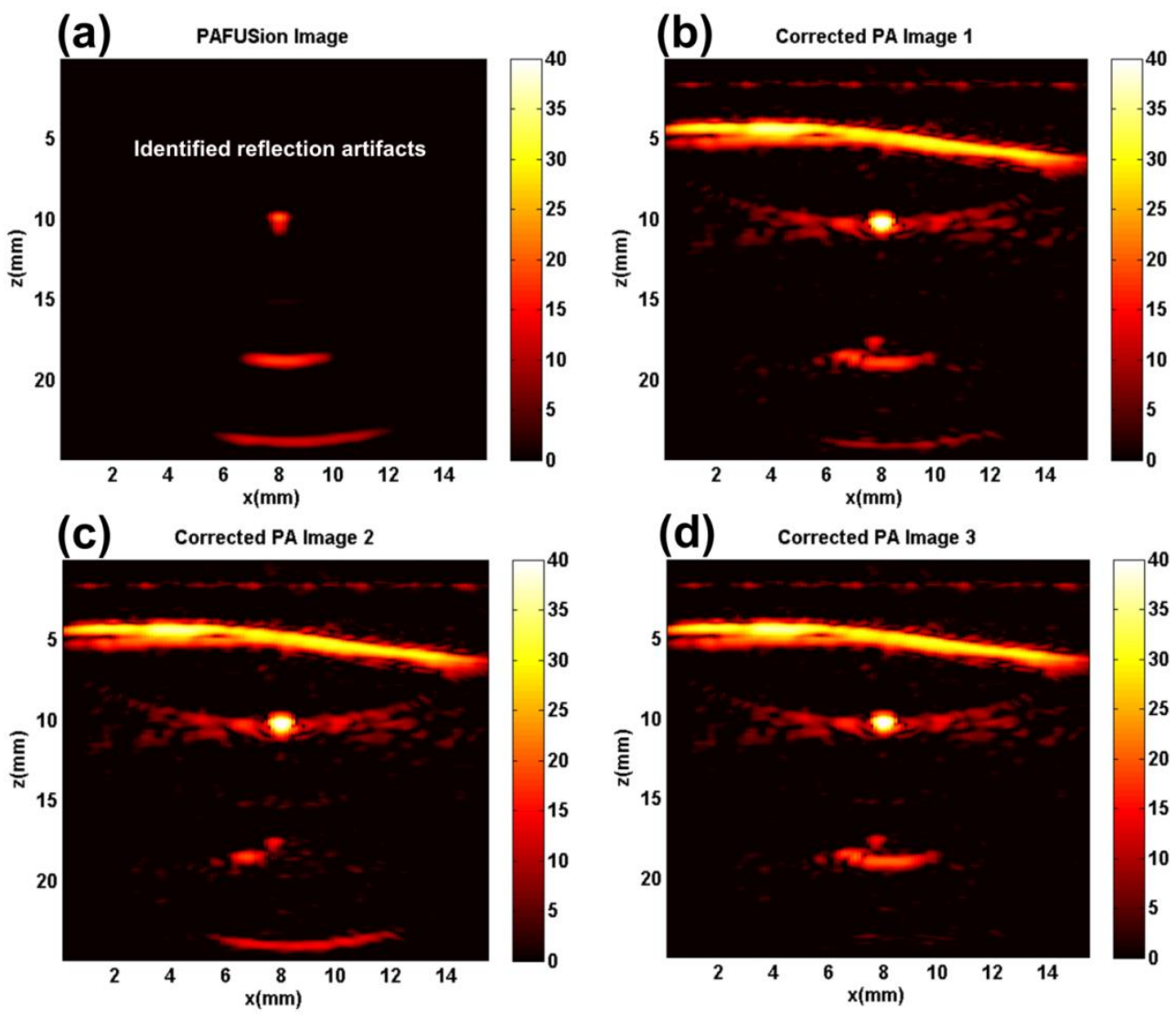

Figure 5.6 Synthetic aperture PAFUSion to identify and reduce reflection artifacts in the phantom experiment: (a) PAFUSion image, (b) corrected PA image in which the pixel intensities of the feature $\mathrm{a}_{3}$ is reduced, (c) corrected PA image in which the pixel intensities of the feature as is reduced, (d) corrected PA image in which the pixel intensities of the feature $\mathrm{a}_{6}$ is reduced.

\begin{tabular}{|c|c|c|c|c|c|c|}
\hline Region of interest & $\mathbf{a}_{\mathbf{1}}$ & $\mathbf{a}_{\mathbf{2}}$ & $\mathbf{a}_{3}$ & $\mathbf{a}_{\mathbf{4}}$ & $\mathbf{a}_{5}$ & $\mathbf{a}_{\mathbf{6}}$ \\
\hline $\begin{array}{c}\text { IRR (dB) } \\
\text { (Plane-wave) }\end{array}$ & 1 & 1.1 & 15 & 1 & 12 & 14.2 \\
\hline $\begin{array}{c}\text { IRR (dB) } \\
\text { (Synthetic aperture) }\end{array}$ & 1 & 1.15 & 15.2 & 1 & 13.5 & 13.1 \\
\hline
\end{tabular}

Table 5.1 Comparison of Intensity Reduction Ratios (IRR) achieved using plane-wave and synthetic aperture PAFUSion for the phantom measurement. 


\subsubsection{In vivo experiment}

In vivo evaluation and comparison of plane-wave and synthetic aperture PAFUSion was performed on the index finger of a human volunteer. Figure 5.7a shows the PA image of the finger in which several bright features are visible. Some of the features are marked in the PA image for reference in Figure 5.7c. The high PA response from the skin melanin layer is visible as a bright arc-like feature (marked as $\mathrm{a}_{1}$ ) at a depth of around $\mathrm{z}=7 \mathrm{~mm}$. Four bright features $\left(a_{2}, a_{3}, a_{4}\right.$, and $\left.a_{5}\right)$ are visible just underneath the skin layer which might be superficial blood vessels. Apart from these, several diffuse features are visible beneath the skin and deeper inside the tissue, out of which some are marked ( $a_{6}, a_{7}, a_{8}$, and $\left.a_{9}\right)$. Figure $5.7 b$ shows the synthetic aperture US image of the same location. Skin $(z=7 \mathrm{~mm})$, bone $(z=10 \mathrm{~mm})$ and several other echogenic structures are visible in US image.
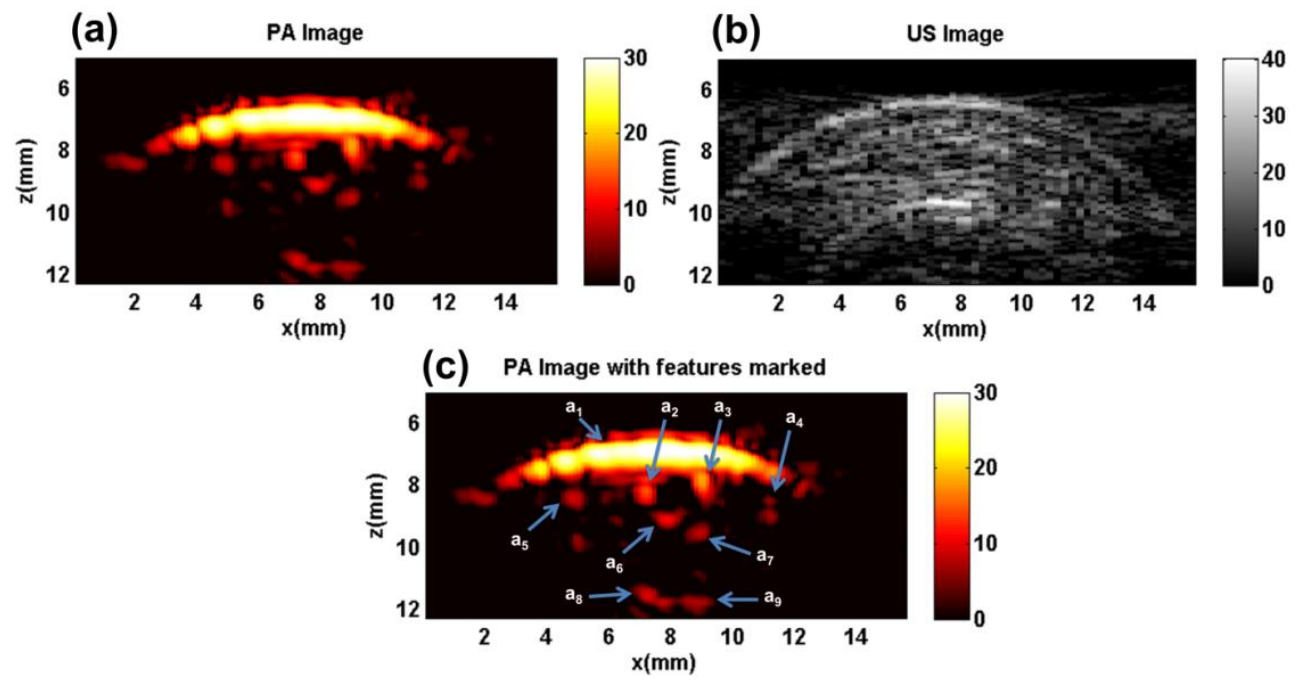

Figure 5.7 In vivo experiment results: (a) PA image, (b) US image, (c) PA image with several features marked $\left(a_{1}-a 9\right)$.

Figure 5.8 shows the results of using plane-wave PAFUSion for identifying and reducing reflection artifacts in the in vivo experiment. The PAFUSion image (Figure 5.8a) shows the reflection artifacts and demonstrates that the majority of the features in the PA image correlate with the simulated reflections. Except for $\mathrm{a}_{1}-\mathrm{a}_{4}$, all other features in the PA image are exposed as reflection artifacts by using plane-wave PAFUSion. As in the phantom experiment, artifacts are identified at the depth of the skin melanin layer $(\mathrm{z}=7 \mathrm{~mm})$ whose 
relative pixel intensities are though far smaller than the actual PA signal from the skin. As hypothesized in a previous study and in the phantom experiment, this is most likely an artifact of the backpropagation approach. Most of the identified artifacts are in close proximity to superficial blood vessels. In an uncorrected image, features like $\mathrm{a}_{5}-\mathrm{a}_{7}$ could therefore be easily misinterpreted as blood vessels, inflammation or a cancerous tissue.
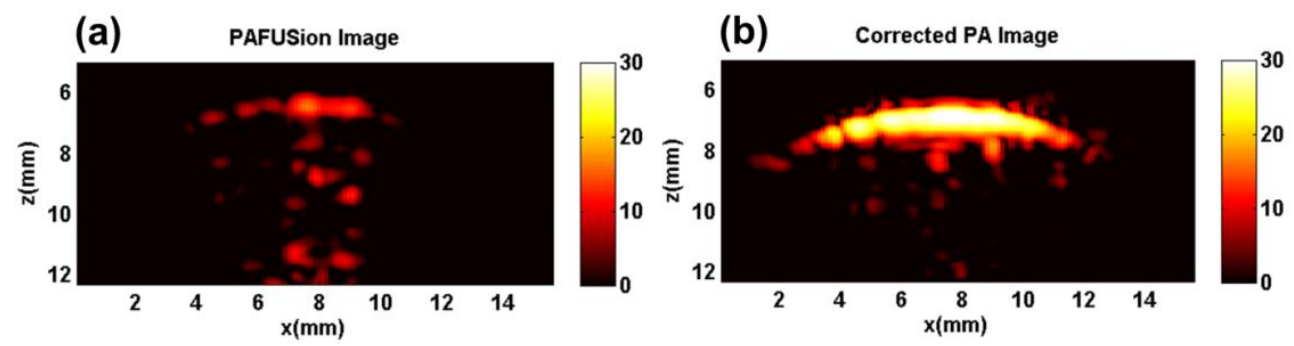

Figure 5.8 Plane-wave PAFUSion to identify and reduce reflection artifacts in the in vivo measurement: (a) PAFUSion image, (b) corrected PA image.

The relative amplitudes and phases of the identified and the actual reflection artifacts are not same. Similar to the phantom experiment, simultaneous correction of all artifacts with a single amplitude and phase matching step was thus not possible. For avoiding multiple images and discussion of results, correction of PA image is demonstrated by one-step amplitude and phase matching, which is adapted for reduction of one of the identified reflection artifacts $\left(\mathrm{a}_{7}\right)$. Figure $5.8 \mathrm{~b}$ shows the corrected PA image in which the pixel intensities of identified artifacts are significantly reduced. Features $\mathrm{a}_{6}-\mathrm{a}_{9}$ almost completely disappeared in the corrected PA image. The intensity of the feature $\mathrm{a}_{5}$ that may be interpreted as a blood vessel also is significantly reduced compared to the PA image. The ratios of PA pixel intensities before and after correction for all the regions marked in the PA image are summarized in Table 5.2 to quantitatively validate the efficiency of plane-wave PAFUSion in reducing reflection artifacts. There is potential to increase the artifact reduction efficiency by performing amplitude and phase matching separately for every identified artifact before image correction step, as demonstrated in the phantom experiment.

Figure 5.9 shows the results of PA reflection artifact reduction achieved at the same location of the finger using synthetic aperture PAFUSion. Figure 5.9a shows the PAFUSion image. It is clear that all the artifacts that were identified by using plane-wave PAFUSion (Figure 5.8a) are identified in this case also. The only difference between the two approaches 
is the amplitudes of the identified artifacts (possibly caused by the motion of finger in between measurements). Figure 5.9b shows the PA image corrected using synthetic aperture PAFUSion in which reflection artifacts are significantly reduced. Reflection artifact reduction ratio values for all the marked features in the PA image are summarized in $\mathrm{dB}$ scale in Table 5.2 to quantitatively validate and compare the efficiency of synthetic aperture PAFUSion with plane-wave PAFUSion in reducing reflection artifacts.
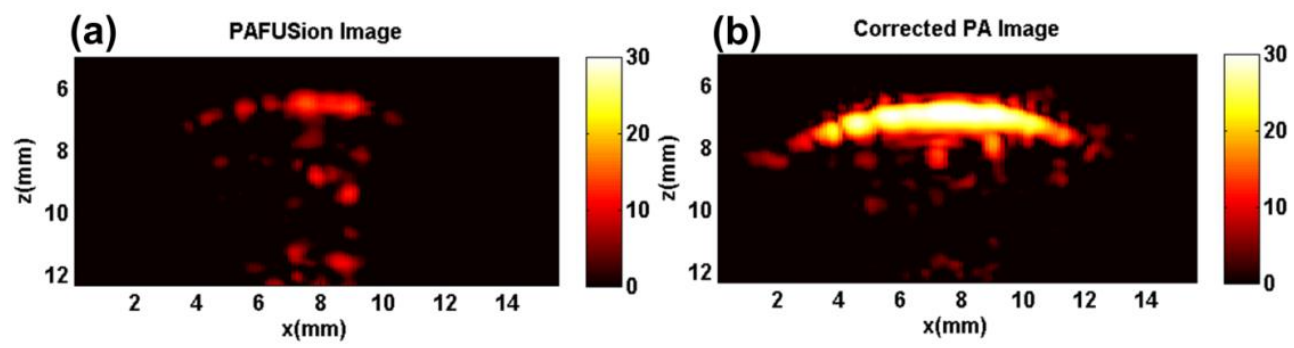

Figure 5.9 Synthetic aperture PAFUSion to identify and reduce reflection artifacts in the in vivo measurement: (a) PAFUSion image, (b) corrected PA image.

Table 5.2 demonstrates that the pixel intensities of features $a_{5}$ to a are significantly reduced in the corrected PA image using both the approaches, with an average reduction of around $13 \mathrm{~dB}$, substantiating the fact that these are reflection artifacts. The intensities of features $\mathrm{a}_{1}$ to $\mathrm{a}_{4}$ are reduced only by around $1 \mathrm{~dB}$ which is significantly less than what was measured for $a_{5}$ to $a_{9}$, substantiating the hypothesis that these are real PA sources. Slight differences are evident in the corrected PA images and consequently the intensity reduction ratio for both approaches (especially for features as to a9). Most likely, this would have been caused by slight movement of the volunteer's finger during the collection of synthetic aperture US data which was the last acquisition step in the measurement chain.

\begin{tabular}{|c|c|c|c|c|c|c|c|c|c|}
\hline Region of interest & $\mathbf{a}_{\mathbf{1}}$ & $\mathbf{a}_{\mathbf{2}}$ & $\mathbf{a}_{3}$ & $\mathbf{\mathbf { a } _ { 4 }}$ & $\mathbf{\mathbf { a } _ { 5 }}$ & $\mathbf{a}_{\mathbf{6}}$ & $\mathbf{a}_{7}$ & $\mathbf{a}_{\mathbf{8}}$ & $\mathbf{a}_{9}$ \\
\hline $\begin{array}{c}\text { IRR (dB) } \\
\text { (Plane-wave) }\end{array}$ & 1.3 & 1.1 & 1 & 1 & 5 & 14 & 16 & 18 & 20 \\
\hline $\begin{array}{c}\text { IRR (dB) } \\
\text { (Synthetic aperture) }\end{array}$ & 1.2 & 1.2 & 1 & 1 & 3.5 & 10 & 16.2 & 14 & 14.5 \\
\hline
\end{tabular}

Table 5.2. Comparison of Intensity Reduction Ratios (IRR) achieved using plane-wave and synthetic aperture PAFUSion for the in vivo measurement. 


\subsection{Discussion}

The phantom and the in vivo results demonstrated that both the plane-wave and the synthetic aperture backpropagation-based PAFUSion are capable of identifying and significantly reducing reflection artifacts in epi-mode PA imaging using a commercial US scanner and an integrated US/PA probe. With both the approaches, reflection artifacts were reduced on an average by $13 \mathrm{~dB}$ in both the phantom and the in vivo studies. We demonstrated identification and strong reduction of reflection artifacts in superficial tissue and near the bone surface of the finger. In a conventional image, these artifacts may well be misinterpreted as small blood vessels, influencing the diagnosis of e.g. inflammation in rheumatoid arthritis, thus demonstrating the importance of PAFUSion in identifying and reducing them.

The goal of this paper was to quantitatively compare the plane-wave and the synthetic aperture approach. As hypothesized in the theory section, our results give a direct confirmation that the plane-wave PAFUSion requiring less acquisitions is more suited for reduction of reflection artifacts in clinical epi-PA imaging, when the skin absorption is the main cause of reflection artifacts (skin mimicking layer/skin was the most important artifact source in phantom and in vivo study respectively). Based on the hypothesis that superposition of a sparse number of plane transients may be sufficient to mimic most part of the PA wavefield of a skin layer, plane-wave PAFUSion was performed with only 33 acquisitions (33 angles) compared to 64 acquisitions (scan through all 64 elements and one element transmitting at a time) used in the synthetic aperture approach. In a real-time scenario, this will already increase the frame rate by almost 2 times when using the plane-wave approach.

If the skin layer is less curved in the PA image, an even smaller angle range and thus a smaller number of plane-waves will be sufficient to mimic the inward propagating wavefield from the skin. In an extreme case, if the skin is a straight-line feature, then plane-wave PAFUSion can be done with a single US plane-wave transmitted at an angle perpendicular to that feature. However, to determine the minimum number of angles required to mimic inward propagating wavefield of the skin melanin layer in an average situation, it requires further investigation. The efficiency of reducing reflection artifacts (intensity reduction ratio) that were caused by both the extended features and point-like absorbers in the phantom experiment were found to be similar for both approaches. In any clinical application of epimode PA imaging, skin is considered to be the most important artifact source because of the 
high light fluence on the skin surface. Under this circumstance, plane-wave PAFUSion will be an appropriate choice because it is more favorable in terms of acquisition speed required for freehand clinical applications. On the other hand, if the main reflection artifact source is a point-like absorber as in interstitial PA imaging (high PA signal from/near the optical fiber/needle tip causing reflection artifacts) [19-21], synthetic aperture PAFUSion will be the better choice, because the full range of the angular aperture of the probe is required to mimic as much as possible of the inward propagating wavefield from a point-like PA source. If the point source is located near the probe, only a sub-aperture of all probe transducer elements the ones that are located within the probe's angular aperture - can effectively contribute to backpropagation towards the point source. In this case, the number of elements required for the element-by-element approach may even be smaller than the number of required angles for the plane wave approach. However, prior knowledge about the position of the PA source (can be obtained with the guidance of PA and US data) is essential to select the required set of elements for doing synthetic aperture PAFUSion in this situation.

Both in the phantom and in vivo measurements (plane-wave and synthetic aperture PAFUSion), the relative amplitudes and phases of the actual and the identified reflection artifacts were found to be different. Consequently, a correction with one-step amplitude and phase matching for all features was not possible in this study. A reason for relative amplitude and phase difference could be that - because the distance to the skin/phantom surface is small (5-10 mm) - the to-be-simulated PA transients are not in the elevation focal distance of the probe $(24 \mathrm{~mm})$ which results in a distance-dependence of the pressure amplitudes. In this situation, scaling of pressure amplitudes based on the distance from the geometrical focus of the probe may be required to avoid the problem of relative amplitude difference when using PAFUSion. A single amplitude and phase matching step was used for the in vivo study (for example: Figure 5.8b shows corrected PA image obtained with one amplitude and phase matching step). In this case, artifacts are strongly reduced, while the intensity of real PA features remains basically unchanged. Therefore we hypothesize that it will be possible to adaptively choose a spatially dependent matching function for amplitude and phase by optimizing local image contrast. In a clinical situation, the PAFUSion image itself will already be relevant for identifying true features in the PA image. However, reduction of reflection artifacts in the PA image has the added benefit of identifying true PA features that are else hidden by reflection artifacts. The approach of matching the amplitude/phase of each 
reflection artifact separately and correcting the artifacts one by one real-time during the measurement is also a feasible option. For example, clinicians can be given an option to click on features in the PAFUSion image (identified artifacts) and automatic correction of that particular feature by suitable scaling of pixel intensities and phases can be potentially implemented. Alternatively, the image can be split into sub-regions in each of which an automatic correction is performed.

The implementation of plane-wave and synthetic aperture PAFUSion is straightforward in any system with sufficient transmission and acquisition control. Even though PAFUSion processing is done offline in this study, real-time implementation of plane-wave and synthetic aperture PAFUSion is feasible in any US/PA system. The system used for this study can perform interleaved US and PA measurements at a high frame rate $(200 \mathrm{~Hz}$ limited by maximum permissible light exposure) and we envisage implementing real-time PAFUSion in this system for future clinical studies.

\subsection{Conclusions}

In summary, a comparison of plane-wave and synthetic aperture PAFUSion in a commercial US scanner used in combination with an integrated US/PA probe demonstrates strong reduction of in-plane reflection artifacts (average reduction of $13 \mathrm{~dB}$ ) in phantom and in vivo measurements using both approaches. Our results demonstrate that plane-wave PAFUSion requiring less number of acquisitions holds more potential for reduction of reflection artifacts in clinical epi-PA imaging, in situations where the skin absorption is the main cause of reflection artifacts. 


\subsection{References}

1. Y. Zhou, J. Yao, and L.V. Wang, Tutorial on photoacoustic tomography. J Biomed Opt, 2016. 21(6): p. 61007.

2. P. Beard, Biomedical photoacoustic imaging. Interface Focus, 2011. 1(4): p. 60231.

3. V. Ntziachristos, Going deeper than microscopy: the optical imaging frontier in biology. Nature Methods, 2010. 7(8): p. 603-614.

4. M. Heijblom, et al., The state of the art in breast imaging using the Twente Photoacoustic Mammoscope: results from 31 measurements on malignancies. European Radiology, 2016: p. 1-14.

5. S.A. Ermilov, et al., Laser optoacoustic imaging system for detection of breast cancer. Journal of Biomedical Optics, 2009. 14(2).

6. P. van Es, et al., Initial results of finger imaging using photoacoustic computed tomography. J Biomed Opt, 2014. 19(6): p. 60501.

7. X.L. Dean-Ben and D. Razansky, Adding fifth dimension to optoacoustic imaging: volumetric time-resolved spectrally enriched tomography. Light-Science \& Applications, 2014. 3.

8. A. Hussain, et al., Quantitative blood oxygen saturation imaging using combined photoacoustics and acousto-optics. Optics Letters, 2016. 41(8): p. 1720-1723.

9. M. Kuniyil Ajith Singh, W. Steenbergen, and S. Manohar, Handheld Probe-Based Dual Mode Ultrasound/Photoacoustics for Biomedical Imaging, in Frontiers in Biophotonics for Translational Medicine, M. Olivo and U.S. Dinish, Editors. 2016, Springer Singapore. p. 209-247.

10. M. Jaeger, J.C. Bamber, and M. Frenz, Clutter elimination for deep clinical optoacoustic imaging using localised vibration tagging (LOVIT). Photoacoustics, 2013. 1(2): p. 19-29.

11. M. Jaeger, et al., Deformation-compensated averaging for clutter reduction in epiphotoacoustic imaging in vivo. Journal of Biomedical Optics, 2012. 17(6).

12. M. Kuniyil Ajith Singh and W. Steenbergen, Photoacoustic-guided focused ultrasound (PAFUSion) for identifying reflection artifacts in photoacoustic imaging. Photoacoustics, 2015. 3(4): p. 123-131.

13. M. Kuniyil Ajith Singh, et al., In vivo demonstration of reflection artifact reduction in photoacoustic imaging using synthetic aperture photoacoustic-guided focused ultrasound (PAFUSion). Biomedical Optics Express, 2016. 7(8): p. 2955-2972.

14. H.M. Schwab, M.F. Beckmann, and G. Schmitz, Photoacoustic clutter reduction by inversion of a linear scatter model using plane wave ultrasound measurements. Biomedical Optics Express, 2016. 7(4): p. 1468-1478.

15. E.J. Alles, M. Jaeger, and J.C. Bamber, Photoacoustic Clutter Reduction Using Short-Lag Spatial Coherence Weighted Imaging. 2014 Ieee International Ultrasonics Symposium (Ius), 2014: p. 41-44.

16. X.L. Dean-Ben, V. Ntziachristos, and D. Razansky, Artefact reduction in optoacoustic tomographic imaging by estimating the distribution of acoustic scatterers. Journal of Biomedical Optics, 2012. 17(11).

17. K. Daoudi, et al., Handheld probe integrating laser diode and ultrasound transducer array for ultrasound/photoacoustic dual modality imaging. Optics Express, 2014. 22(21): p. 26365-26374. 
18. M. Jaeger, et al., Fourier reconstruction in optoacoustic imaging using truncated regularized inverse k-space interpolation. Inverse Problems, 2007. 23(6): p. S51S63.

19. M.A.L. Bell, et al., In vivo visualization of prostate brachytherapy seeds with photoacoustic imaging. Journal of Biomedical Optics, 2014. 19(12).

20. D. Piras, et al., Photoacoustic needle: minimally invasive guidance to biopsy. J Biomed Opt, 2013. 18(7): p. 070502.

21. W. Xia, et al., Performance characteristics of an interventional multispectral photoacoustic imaging system for guiding minimally invasive procedures. J Biomed Opt, 2015. 20(8): p. 86005. 


\section{Photoacoustic-guided}

\section{focused ultrasound for accurate}

\section{visualization of brachytherapy}

\section{seeds with the photoacoustic}

\section{needle $^{5}$}

An important problem in minimally-invasive photoacoustic imaging of brachytherapy seeds is reflection artifacts caused by the high signal from the optical fiber/needle tip reflecting off the seed. The presence of these artifacts confounds interpretation of images. In this letter, we demonstrate a recently developed concept called PAFUSion (photoacoustic-guided focused ultrasound) for the first time in the context of interstitial illumination photoacoustic imaging to identify and remove reflection artifacts. In this method, ultrasound from the transducer is focused in the region of the optical fiber/needle-tip identified in a first step using photoacoustic imaging. The image developed from the ultrasound diverging from the focus zone at the tip region visualizes only the reflections from seed and other acoustic inhomogeneities, allowing identification of the reflection artifacts of the first step. These artifacts can then be removed from the photoacoustic image. Using PAFUSion, we demonstrate reduction of reflection artifacts and thereby improved interstitial photoacoustic visualization of brachytherapy seeds in phantom and ex vivo measurements on porcine tissue.

\footnotetext{
5 Part of this chapter has been accepted for publication as: M. Kuniyil Ajith Singh, V. Parameshwarappa, E. Hendriksen, W. Steenbergen, and S. Manohar; "Photoacoustic-guided focused ultrasound for accurate visualization of brachytherapy seeds with the photoacoustic needle," Journal of Biomedical Optics (2016).
} 


\subsection{Introduction}

Prostate cancer is one of the most common forms of cancer among men [1]. Depending on the stage and severity of prostate cancer, several therapeutic options like radiation therapy, hormone therapy and radical prostatectomy are generally used. Brachytherapy is a targeted radiation therapy treatment which enables high-precision delivery of radiation doses to the tumour area in the prostate [2]. In brachytherapy, the localized tumour area instead the whole prostate is targeted with defined radiation dose, using multiple (50-100) tiny metallic radioactive sources implanted inside the prostate.

Brachytherapy is efficient and more convenient for the patient as the treatment is a single implantation procedure in the operation theatre instead of an external beam radiation course up to 7.5 weeks [2]. However, complications may arise when there is a deviation between planned and delivered radiation dose [3]. Potential reasons for this deviation are seed migration, prostate motion, oedema, or implantation errors [3]. Mismatch between planned and delivered radiation dose can be avoided to a large extent by accurate visualization of seeds during the implantation procedure [2]. Real-time seed visualization during the implantation procedure is currently performed using transrectal ultrasound (TRUS) imaging. However, seeds are often challenging to visualize using ultrasound (US) due to their small size, their similarity in acoustic impedance with micro-calcifications, and due to presence of characteristic acoustic artifacts like shadowing and reverberations [4]. After the implantation procedure, seed locations are confirmed conventionally using post-operative computed tomography [5]. This also helps in determining whether any additional radiation therapy is required to compensate for the underdosed region in the prostate. However, this approach with increased radiation exposure does not solve the problem of overdosing healthy tissue. Over or underdosage of tissue is less likely if seeds are accurately visualized during the implantation procedure.

In photoacoustic (PA) imaging, pulsed light absorbed by the target emits US by the thermoelastic mechanism [6]. This US is detected using a US detector array to build up an image. PA imaging offers spectroscopic optical absorption contrast with US resolution [7]. Owing to the high optical absorption of metal compared to tissue, PA imaging offers excellent contrast in imaging brachytherapy seeds [4, 8]. PA imaging thus can complement US imaging for better visualization of brachytherapy seeds [4, 8, 9]. 
Considering the imaging depth required, the PA needle $[10,11]$ with interstitial light delivery via a cutting percutaneous needle is generating much interest in imaging brachytherapy seeds [9]. A challenge in interstitial PA imaging of brachytherapy seeds is the presence of reflection artifacts caused by the high PA signal from the optical fiber/needle tip reflecting off the seeds as shown in Figure 6.1b. These artifacts may appear in the region of interest and can reduce the contrast and causes difficulty in image interpretation as reflection artifacts can be misinterpreted as seeds [9]. It is thus important to identify and reduce reflection artifacts for accurate interstitial PA visualization of brachytherapy seeds.

We recently proposed a method called PAFUSion (Photoacoustic-guided focused ultrasound) to identify reflection artifacts in epi-mode PA imaging [12]. In this work, we study the use of PAFUSion in identifying and reducing reflection artifacts with the use of the PA-needle in the visualization of brachytherapy seeds. We present the results of the combined approach in visualizing non-radioactive brachytherapy seeds in a tissue mimicking phantom and an ex vivo porcine tissue. In addition, we also show the potential of using multi-spectral PA for separating seed signals from other optical absorbers like blood in a phantom measurement.

\subsection{Materials and methods}

The system consists of a commercial handheld US imager (MyLab_One, Esaote Europe BV, Maastricht, the Netherlands) with a linear array probe SL3323 (128 elements, 7.5 MHz, 100 $\%$ bandwidth) [13]. The system is used in the research mode with 64 elements where US transmission, laser pulse transmission, and data acquisition are controlled using custom-made software running on a PC. Illumination uses an Nd:YAG laser (Quanta-Ray pro 250, Spectra Physics, Mountain View, California) pumping an optical parametric oscillator (OPO, VersaScan- L532, GWU, Erftstadt, Germany) at $10 \mathrm{~Hz}$. The energy per pulse is monitored using a photodetector (Model 1621, Si, 350-1000 nm, 0.8 mm Diameter, 8-32 / M4, Newport Corporation, California). A multimode optical fiber (NA 0.22 , diameter $600 \mu \mathrm{m}$ ) coupled to the laser delivers $10 \mathrm{~ns}$ laser light with $1.2 \mathrm{~mJ} /$ pulse at $800 \mathrm{~nm}$. For the multi-wavelength studies, the range of $720 \mathrm{~nm}-860 \mathrm{~nm}$ was covered with $5 \mathrm{~nm}$ steps; single wavelength studies used $800 \mathrm{~nm}$. The fiber is held in a biopsy needle $(14 \mathrm{G} \times 13 \mathrm{~cm}$, Bard Ltd, UK) and slide-fits into the $14 \mathrm{G}$ cutting cannula (Figure 6.1a). The PA frame rate is $10 \mathrm{~Hz}$ and images 
can be displayed online using a GPU-based delay and sum reconstruction algorithm. Images presented here are averaged over 100 frames and reconstructed offline using a frequency domain reconstruction algorithm [14], assuming constant speed of sound in the medium for all measurements (phantom measurement: $1480 \mathrm{~m} / \mathrm{s}$, ex vivo measurement: $1540 \mathrm{~m} / \mathrm{s}$ ).
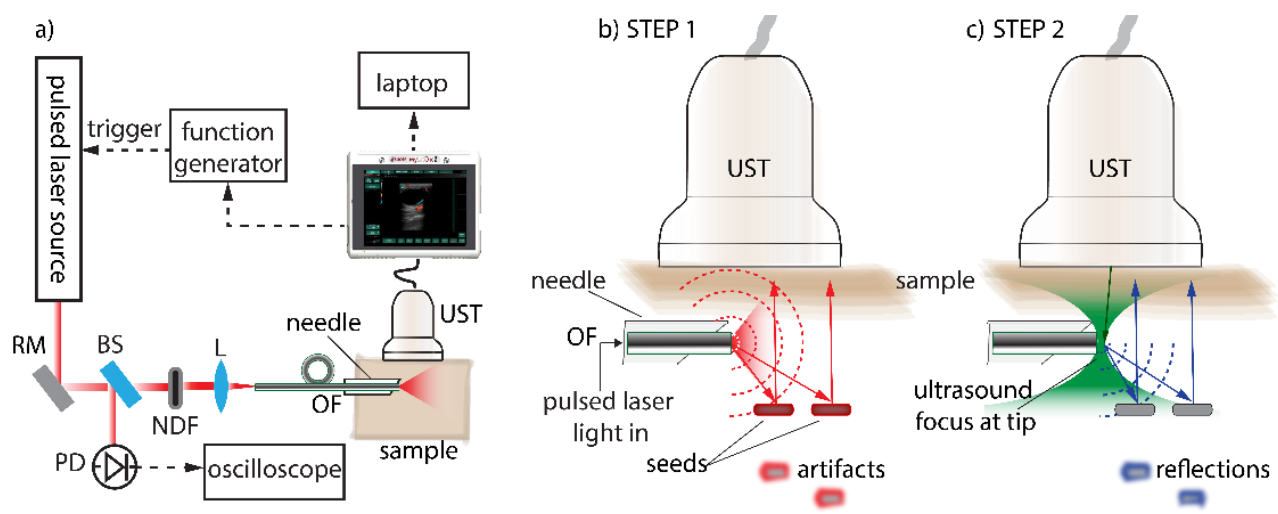

Figure 6.1 (a) Block diagram of setup. RM: reflecting mirror; BS: glass plate to sample beam; PD: photodetector; NDF: neutral density filter; L: lens; OF: optical fiber; UST: ultrasound transducer. (b) Concept of the PA needle, where pulsed light is applied in tissue via an optical fiber in a cutting needle with external US detection. By this, tissue at the optical fiber tip, the tip itself and the brachytherapy seeds can be visualized. Additionally however artifacts are also visualized due to reflection of the high PA signal from the fiber/needle tip on the seeds, (c) Concept of PAFUSion where in a second step; US from the transducer is focused at the optical fiber/needle-tip. The US diverging from the focus zone at the tip is in a first approximation a mimic of the PA signal from tip in the first step. The detected signals visualize only the reflections from seed and other inhomogeneities allowing identification of the reflection artifacts of the first step.

The PAFUSion approach specifically in the context of the PA needle is shown in Figure $6.1 \mathrm{~b}$ and c. Once the needle tip has been identified from the PA image in step 1 (Figure 6.1b), US is focused to this point in a second step. The resultant US data processed using the PAFUSion algorithm [12] generates an image with only reflections from deeper structures (PAFUSion image) hence representing the artifacts in the PA image from step 1. The average image pixel value of the reflection artifact with the highest image value is then scaled to the image value of its counterpart in the PA image, and the same scaling applied to all other 
identified artifacts in the PAFUSion image. After this, reconstructed and enveloped PAFUSion data is subtracted from the PA data and the positive parts of the result are plotted to obtain the corrected PA image. The negative parts of the result are assigned an image value of zero.

Figure 6.2a shows the schematic of the experiment to validate the proposed combined approach in a scattering phantom. The phantom $(10 \mathrm{~cm} \times 7.5 \mathrm{~cm} \times 5 \mathrm{~cm})$ was prepared with Agar dissolved in an aqueous solution of Intralipid (I141, 20\% emulsion, Sigma Aldrich) to provide a bulk reduced scattering coefficient of $\mu_{s}{ }^{\prime}=1 \mathrm{~mm}^{-1}$. Within the phantom, three nonradioactive brachytherapy seeds (length $=4.5 \mathrm{~mm} \times 0.8 \mathrm{~mm}$, Bard Benelux N.V., the Netherlands) were embedded. The seeds were coated with a single layer of India ink $(-200$ $\mu \mathrm{m})$ by dipping them in the ink and drying in air. A single bead ( $2.2 \mathrm{~mm}$ diameter $)$ of sodium alginate carrying gold nanorods (AuNR) was also embedded in the phantom. The AuNR were prepared using a well-known synthesis protocol [15]. The reference absorption spectrum of the bead (Figure 6.2d) shows a characteristic longitudinal plasmon absorption peak at 795 $\mathrm{nm}$. Water-coupling was used in between the US probe and the phantom. The needle was oriented parallel to the US detector array. PA and plane-wave US images are acquired in the first step. Then after locating the fiber/needle tip, PAFUSion (focused US) data was also collected for offline processing. PA images for different excitation wavelengths $(720 \mathrm{~nm}$ to $860 \mathrm{~nm}$ ) were also acquired.

Ex vivo validation of the approach was performed using four brachytherapy seeds (coated with single layer of India ink) embedded inside porcine tissue. Using US guidance, the seeds were implanted inside the tissue using a standard 18G brachytherapy needle (Bard, Brachystar, Covington, Georgia) by one of the authors (E.M). The same data acquisition and processing protocol was followed as for the phantom experiment.

\subsection{Results and discussion}

Figure 6.2b shows the reconstructed PA image (phantom experiment) in which shaft and the fiber/needle tip and all three seeds are visualized. The AuNR bead is seen only with top and bottom surfaces, as expected in a limited-view situation. The US image (Figure 6.2c) shows the needle shaft, and the seeds. In an ideal case, only the optically absorbing structures are expected to show up in the reconstructed PA image. However, a further feature is visible in 
the PA image (lateral distance $=9.5 \mathrm{~mm}$, depth $=24 \mathrm{~mm}$ ) which could be interpreted as an optically absorbing feature; for example another seed.

The PAFUSion image (Figure 6.2e) reveals this feature as a reflection artifact. Figure 6.2f shows the corrected PA image obtained by subtracting the PAFUSion-identified reflection artifacts from the real PA image showing a reduction of the artifact. The image values of real PA features are unaffected in the corrected PA image. In Figure 6.2d, the PA image values of seed 1 and that of the AuNR bead (normalized to the measured pulse energy) are plotted against the wavelength, together with the reference spectrum of AuNR. The image values of seeds coated with India ink decreases monotonically with wavelength, while the PA spectrum of the bead shows the signature longitudinal plasmon peak at roughly $795 \mathrm{~nm}$.
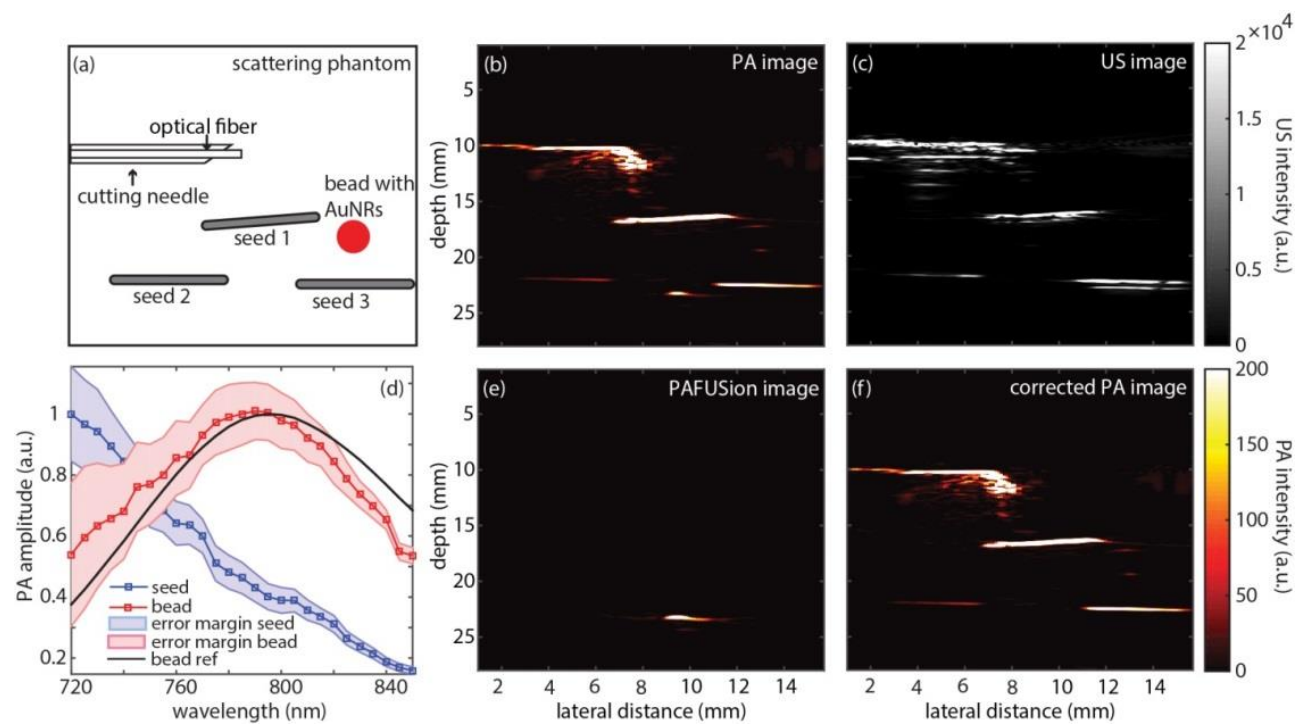

Figure 6.2 (a) Schematic of the experiment showing orientation of the PA-needle, arrangement of seeds and AuNR bead in the Agar phantom with Intralipid, (b) PA image at a wavelength of $800 \mathrm{~nm}$ showing the expected features, and with an additional feature at $9.5 \times 24 \mathrm{~mm}$ (lateral distance x depth), (c) plane-wave US image, (d) measured PA spectrum of seed 1, bead with error margins (standard deviation of the averaged mean) compared with reference spectrum of the bead, (e) PAFUSion image showing that the unknown feature is a reflection artifact, (f) corrected PA image obtained by subtracting scaled PAFUSion image from the PA image. 
Figure 6.3a shows the schematic of the arrangement of seeds inside the ex vivo porcine tissue and the orientation of PA-needle in the measurement setup. For better acoustic coupling, the sample was covered with transparent kitchen cling film $(-20 \mu \mathrm{m})$ after smearing with US gel (Figure 6.3d). Figure 6.3b shows the reconstructed PA image in which PA fiber/needle tip, all four seeds, and several other bright features are visible. It is difficult to interpret this image since several bright features with shapes similar to the seeds are present. The shaft of the PA-needle, all the four seeds and several other echogenic tissue structures are visible in the US image (Figure 6.3c).
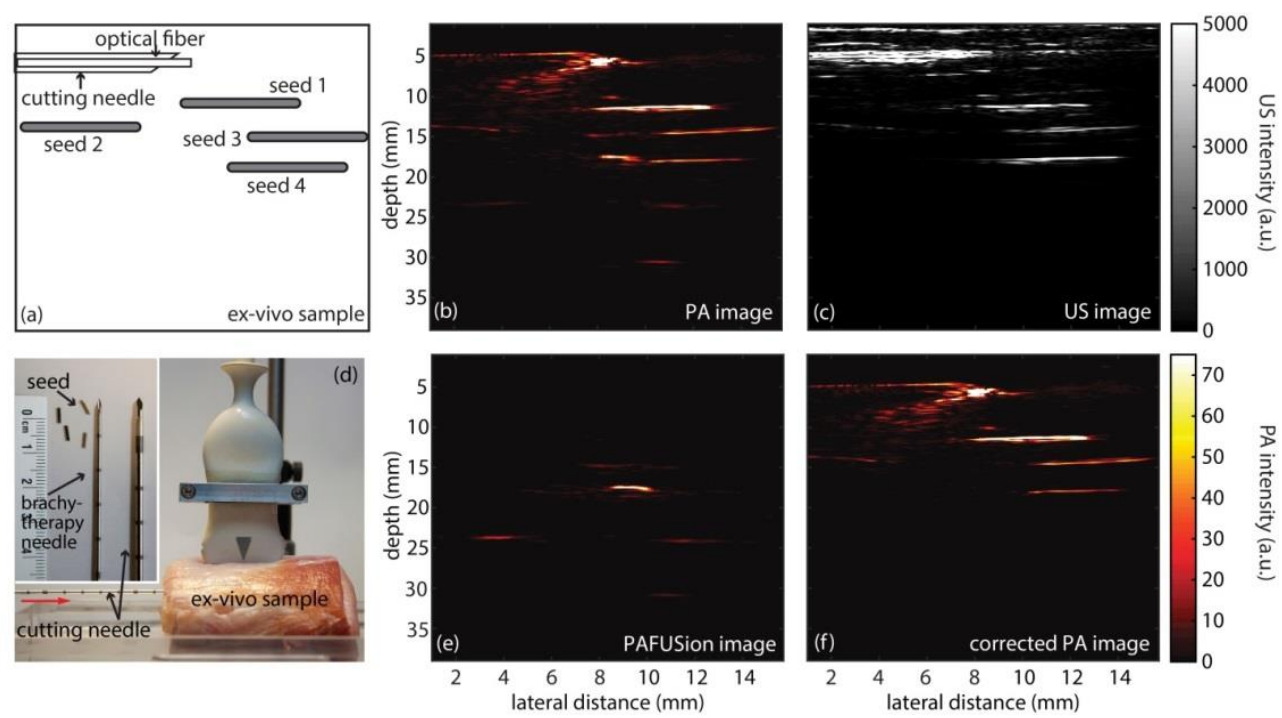

Figure 6.3 (a) Schematic showing orientation of the PA-needle and arrangement of seeds inside the porcine tissue, (b) PA image at a wavelength of $800 \mathrm{~nm}$, (c) plane-wave US image, (d) photograph of the imaging situation with needle inserted in the ex vivo sample. Inset is a photograph of the inner and outer needles alongside the seed. (e) PAFUSion image with the identified reflection artifacts, (f) corrected PA image obtained by subtracting the scaled PAFUSion image from the PA image.

Figure 6.3e shows the PAFUSion image from which reflection artifacts in the PA image can be identified. The PAFUSion image shows that a number of features in the PA image are reflection artifacts. All of them are caused by fiber/needle tip PA signal reflecting off the seeds, and off other tissue echogenic structures. Figure 6.3f shows the corrected PA image obtained by subtracting the PAFUSion-identified reflection artifacts from the real PA image. 
It is evident that the reflection artifacts are significantly reduced by using PAFUSion without affecting the contrast of real PA features. An interesting additional feature is visible at the same depth of seed 3 in the PA image (lateral distance $=7.5 \mathrm{~mm}$, depth $=15 \mathrm{~mm}$ ). The PAFUSion image uncovered this feature as a reflection artifact. This would have been caused by the fiber/needle tip signal reflecting off the point-like tissue feature visible just above the seed 1 (lateral distance $=7.5 \mathrm{~mm}$, depth $=10 \mathrm{~mm}$ ) in both the PA and US image.

Our approach has been successfully demonstrated in phantom and in ex vivo settings. The method has been developed in the context of PA-needle imaging of brachytherapy seeds, but is eminently suited for all applications of the PA-needle where reflection artifacts could result due to the presence of acoustic reflectors. However, the approach needs to be further investigated. One issue is that high absorption of heterogeneous tissue proximal to the needletip could result in multiple bright PA features near the tip. This may create uncertainties in accurately identifying the needle/fiber tip which is essential for using the approach. One solution to this may be scanning the US focus to the discrete high intensity PA regions in turn, and combining all the resultant PAFUSion images to identify the reflection artifacts. Another approach can be physically time-reversing the PA signals acquired from and near the fiber/needle tip to perform the insonification step in the PAFUSion method.

Another issue that requires investigation is the approach used for correction of the PA images. Subtraction of the PAFUSion images from PA image worked well in the situations studied, but will require optimization in the case of real PA features mixed with any reflection artifacts.

\subsection{Conclusions}

We conclude that PAFUSion enables identification and substantial reduction of reflection artifacts when using the PA-needle, considerably improving the visualization of brachytherapy seeds. Our phantom results also confirm that multi-spectral PA can be potentially a useful tool to separate signals from the brachytherapy seeds and other optical absorbers in the tissue. The spectral behavior of the brachytherapy seeds in the phantom experiment may be the result of ink coating, which was used for the contrast enhancement. However, it is well known that bare seeds also possess a similar spectral behavior with drop in PA amplitude with respect to wavelength. The PAFUSion approach reported here is 
suitable for real-time implementation since it requires only a single focus US measurement and an image subtraction step. In a future clinical scenario, we envisage that the PA-needle in combination with real-time PAFUSion will provide accurate visualization of the distribution of brachytherapy seeds, which in turn provide feedback to the radiotherapist regarding adherence to the therapy plan. 


\subsection{References}

1. J. Crook, The role of brachytherapy in the definitive management of prostate cancer. Cancer Radiother, 2011. 15(3): p. 230-7.

2. A.J. Chang, et al., High-risk prostate cancer-classification and therapy. Nature Reviews Clinical Oncology, 2014. 11(6): p. 308-323.

3. E.M. Messing, et al., Intraoperative optimized inverse planning for prostate brachytherapy: early experience. Int J Radiat Oncol Biol Phys, 1999. 44(4): p. 8018.

4. J.L. Su, et al., Photoacoustic imaging of prostate brachytherapy seeds. Biomedical Optics Express, 2011. 2(8): p. 2243-2254.

5. S. Nag, et al., The American Brachytherapy Society recommendations for permanent prostate brachytherapy postimplant dosimetric analysis. International Journal of Radiation Oncology Biology Physics, 2000. 46(1): p. 221-230.

6. Y. Zhou, J. Yao, and L.V. Wang, Tutorial on photoacoustic tomography. J Biomed Opt, 2016. 21(6): p. 61007.

7. M. Kuniyil Ajith Singh, W. Steenbergen, and S. Manohar, Handheld Probe-Based Dual Mode Ultrasound/Photoacoustics for Biomedical Imaging, in Frontiers in Biophotonics for Translational Medicine, M. Olivo and U.S. Dinish, Editors. 2016, Springer Singapore. p. 209-247.

8. T. Harrison and R.J. Zemp, Coregistered photoacoustic-ultrasound imaging applied to brachytherapy. Journal of Biomedical Optics, 2011. 16(8).

9. M.A.L. Bell, et al., In vivo visualization of prostate brachytherapy seeds with photoacoustic imaging. Journal of Biomedical Optics, 2014. 19(12).

10. D. Piras, et al., Photoacoustic needle: minimally invasive guidance to biopsy. J Biomed Opt, 2013. 18(7): p. 070502.

11. W. Xia, et al., Performance characteristics of an interventional multispectral photoacoustic imaging system for guiding minimally invasive procedures. J Biomed Opt, 2015. 20(8): p. 86005.

12. M. Kuniyil Ajith Singh and W. Steenbergen, Photoacoustic-guided focused ultrasound (PAFUSion) for identifying reflection artifacts in photoacoustic imaging. Photoacoustics, 2015. 3(4): p. 123-131.

13. K. Daoudi, et al., Handheld probe integrating laser diode and ultrasound transducer array for ultrasound/photoacoustic dual modality imaging. Optics Express, 2014. 22(21): p. 26365-26374.

14. M. Jaeger, et al., Fourier reconstruction in optoacoustic imaging using truncated regularized inverse k-space interpolation. Inverse Problems, 2007. 23(6): p. S51S63.

15. R.G. Rayavarapu, et al., Synthesis and bioconjugation of gold nanoparticles as potential molecular probes for light-based imaging techniques. Int J Biomed Imaging, 2007. 2007: p. 29817. 


\section{Summary and outlook}

This thesis described a novel method called PAFUSion (photoacoustic-guided focused ultrasound), for identification and elimination of reflection artifacts, an important problem in biomedical photoacoustic imaging. This chapter summarizes the conducted research described in this thesis and gives recommendations for future work to improve upon the proposed technique. The summary of each research aspect will be addressed separately. 


\subsection{Summary}

This section summarizes the research conducted and reported in this thesis. Each research aspects covered in the thesis is discussed separately.

\subsubsection{Photoacoustic-guided focused ultrasound (PAFUSion) to mimic photoacoustic wavefield from point-like optical absorbers and thereby identifying reflection artifacts}

In general, PAFUSion uses ultrasound (US) transmissions to mimic the inward propagating photoacoustic (PA) wavefield (wavefield traversing into the tissue) from optical absorbers and reflection artifacts caused by them. In chapter 3, proof-of-principle of this idea was demonstrated by using focused US to mimic PA wavefields from point-like optical absorbers and thereby identifying reflection artifacts caused by them. In this approach, we transmit a focused US pulse, with the focal position adjusted to the location of the optical absorber by using guidance of PA data. The focused US pulse, at the time of arrival in the focal position, mimics the part of the PA wave that was traversing towards the reflector. Under the assumption that signal acquisition starts at the time when the focused US pulse arrives at the PA source $(t=0)$, the resulting signal will show the US reflections at the same time where they show up in the PA signal. When reconstructing a PA image from the US data, it will thus mimic the reflection artifacts in terms of shape and depth without containing the real PA signal itself.

We performed numerical simulations to validate the idea of PAFUSion for identifying reflection artifacts in PA imaging. 3D simulation of the idea was performed in the k-Wave toolbox by defining a digital phantom with one PA source and an acoustic reflector beneath it to generate reflections. Results demonstrate that the proposed method using focused US can identify reflection artifacts caused by point-like optical absorbers.

To experimentally validate the idea of PAFUSion using focused US, we presented tissuemimicking phantom experiments using a handheld dual mode US/PA system. Results show that: 1) PAFUSion using focused US can uncover in-plane reflection artifacts caused by point-like absorbers in epi-mode PA imaging, 2) shape and spatial details of the identified artifacts are matching quite well with the actual PA artifacts, 3) reflection artifacts can come in close proximity to an optical absorber and PAFUSion can separate them. However, 
reflection artifact reduction was not demonstrated in this proof-of-principle study. Relative image intensities and phases of the identified reflection artifacts (PAFUSion image) were not exactly matching with the PA artifacts, which made the correction of artifacts difficult. In addition, identification of reflection artifacts caused by spatially extended features like skin, requires further improvements to the focusing approach used in this study.

Considering the impact and importance of reflection artifacts in epi-mode biomedical PA imaging and the results using PAFUSion, it is clear from this proof-of-principle study that PAFUSion holds potential in improving PA imaging of acoustically inhomogeneous tissue.

\subsubsection{Synthetic aperture photoacoustic-guided focused ultrasound (PAFUSion) for identification and elimination of reflection artifacts in epi-mode biomedical photoacoustic imaging}

In view of the spatially distributed PA sources that are found in clinical applications, an improved version of PAFUSion was proposed in chapter 4, in which PA signals were backpropagated to imitate the inward travelling wavefield and thus the reflection artifacts. The backpropagation was performed in a synthetic way based on the pulse-echo acquisitions after transmission on each single element of the transducer array.

If it was feasible to re-transmit the PA signal that was detected, this can lead to the reproduction of the reflection artifacts that were contained in the PA signal. Subtraction of these artifacts from the original PA data can result in reflection-artifact reduction. However, physical backpropagation requires immediate re-transmission of detected PA signals and the state-of-the-art US systems will not be able to transmit arbitrary wavefronts. This was the motivation to implement virtual backpropagation of PA signals using post processing of synthetic aperture US data. In this chapter, we showed that reflection artifacts can be identified by convolving time inverted PA signals from a particular element with US data collected by transmission of US on that element and superposing the results obtained for all the elements. After this step, shapes of the identified artifacts were matched with the shape of the real PA artifacts using impulse response matching filters and correction was performed by subtracting identified artifacts from the original PA data.

The proposed approach was validated using phantoms and in vivo measurements on forearm of human volunteers. The phantom as well as the in vivo results demonstrated that synthetic aperture PAFUSion is capable of identifying and strongly reducing reflection 
artifacts in epi-mode PA imaging. Using this technique, the reflection artifact intensity was reduced by around $13 \mathrm{~dB}$ on average in both phantom and in vivo measurements. For the first time, in this chapter, we demonstrated identification and strong reduction of a large number of reflection artifacts just beneath the skin and in proximity to superficial vasculature. In a conventional image, these artifacts may well be misinterpreted as small blood vessels, inflammations or a cancerous tissue, thus demonstrating the importance of PAFUSion in identifying and reducing them. In both the phantom and in vivo results, relative amplitudes and phases of identified reflection artifacts were matching quite well with the actual PA artifacts, which resulted in efficient correction of reflection artifacts. Any clinical applications of PA imaging could benefit from the efficient reflection artifact reduction achieved using synthetic aperture PAFUSion. In summary, the results in this chapter provide a direct confirmation that reflection artifacts are prominent in clinical epi-PA imaging, and that PAFUSion can strongly reduce these artifacts to improve image interpretation, contrast, and imaging depth.

The implementation of virtual PA backpropagation based on reference pulse-echo data is completely software based, which makes implementation of this method in a clinical system straightforward. In this study, the method was implemented in a Verasonics research US system and the frame rate achieved was one image in every three seconds. The framerate was limited by the speed of the laser used and the CPU based implementation in Matlab.

\subsubsection{Photoacoustic reflection artifact reduction using plane-wave and synthetic aperture photoacoustic-guided focused ultrasound (PAFUSion): Evaluation and comparison using a handheld epi-mode ultrasound/photoacoustic imaging system}

In the case where skin absorption is the main cause of reflection artifacts (as in any epi-mode system), the strong PA transient generated by the skin is close to a plane transient, and therefore a superposition of a sparse number of plane transients may be sufficient to mimic most part of the inward travelling PA wavefield. This hypothesis inspired the implementation of an alternative approach where virtual backpropagation is based on US plane-wave acquisitions with different transmit steering angles instead of the synthetic aperture US transmissions as proposed in chapter 4 . We implemented plane-wave and synthetic aperture PAFUSion in a handheld US/PA imaging system and demonstrated reduction of reflection 
artifacts in phantoms and in vivo measurement on a human finger using both approaches in chapter 5. Results suggest that, while both approaches are equivalent in terms of artifact reduction efficiency (13 $\mathrm{dB}$ artifact reduction), plane-wave PAFUSion requires less pulse echo acquisitions when high PA signal from the skin is the main cause of reflection artifacts.

In both the phantom and in vivo results obtained using plane-wave and synthetic aperture PAFUSion, relative amplitudes and phases of identified reflection artifacts were not similar to the actual PA artifacts. Because of this, matching of amplitudes and phases of different artifacts were performed step-by-step, one at a time to achieve full correction of artifacts in the phantom experiment. For the in vivo study, the amplitude and phase matching was performed for only one of the identified artifacts (one-step subtraction by matching the amplitude and phase of one identified artifact with its counterpart in the PA image). This was done in the presentation of in vivo results to avoid complications in discussing results with numerous images (several artifacts may lead to several corrected PA images).

Even though PAFUSion processing was done offline in this study, real-time implementation of plane-wave and synthetic aperture PAFUSion is feasible in any US/PA system with sufficient transmission/acquisition control. The system used for this study can perform interleaved US and PA measurements at a high frame rate $(200 \mathrm{~Hz}$ limited by maximum permissible light exposure) and we envisage implementing real-time PAFUSion in this system for our future clinical studies.

\subsubsection{Photoacoustic-guided focused ultrasound (PAFUSion) for identifying and reducing reflection artifacts in interstitial photoacoustic imaging}

Interstitial illumination PA imaging is generating much interest for guiding minimally invasive procedures. Reflection artifacts caused by the high signal from or near the optical fiber/needle tip constitute a major problem in interstitial illumination PA imaging of brachytherapy seeds. PA signals from or near the optical fiber/needle tip traverse into tissue and reflect off seeds to generate reflection artifacts. These artifacts may appear in the region of interest and can reduce the contrast and causes difficulty in image interpretation as reflection artifacts can be misinterpreted as seeds.

In chapter 6, we demonstrated the use of PAFUSion using focused US to identify and eliminate reflection artifacts caused by the optical fiber/needle tip in interstitial PA imaging of brachytherapy seeds. In interstitial PA imaging, the main cause of reflection artifacts is 
the high PA signal generated in front of the optical fiber/needle tip, which can be assumed as a point-like absorber. This was the motivation to use the approach of focused US to uncover the reflection artifacts. In this approach, once after identifying the fiber/needle tip, US was focused to this depth and the data was reconstructed based on the PAFUSion algorithm explained in chapter 3 to identify reflection artifacts. After this, the average image pixel value of the reflection artifact with the highest image value was then scaled to the image value of its counterpart in the PA image, and the same scaling applied to all other identified artifacts in the PAFUSion image. After this, reconstructed and enveloped PAFUSion data was subtracted from the PA data and the positive parts of the result were plotted to obtain the corrected PA image. The negative parts of the result were assigned an image value of zero. The method was shown to work successfully in improving seed visualization in phantom and ex vivo measurements on porcine tissue.

The US system used for this study was a handheld commercial US scanner with sufficient access to the transmission and acquisition parameters. The frame rate at which the measurements were done was $10 \mathrm{~Hz}$ (limited by the repetition rate of the laser) and the PAFUSion processing was done offline. As PAFUSion in this context requires only a single focus US measurement and an image subtraction step, real-time implementation of the approach is straightforward in any systems. It is envisaged that the PA-needle in combination with real-time PAFUSion will provide accurate visualization of the distribution of brachytherapy seeds, which in turn can provide feedback to the radiotherapist regarding adherence to the therapy plan.

In addition to imaging of brachytherapy seeds, the proposed concept is eminently suited for all applications of interstitial illumination PA imaging where reflection artifacts could result due to the presence of acoustic reflectors. To the best of our knowledge, reduction of reflection artifacts is demonstrated for the first time in the context of interstitial PA imaging, and we envisage that our approach will help bringing the interstitial illumination PA imaging into the realm of practical use. 


\subsection{Outlook}

Different variants of PAFUSion as demonstrated in this thesis are potentially capable of achieving the goal of real-time reflection-artifact-free biomedical PA imaging. However, to obtain this most desired goal, some further technical improvements and methods are required.

\subsubsection{Enhancing the PAFUSion reflection artifact correction efficiency by utilizing the tissue acoustic attenuation map and the knowledge of distance of structures from the geometrical focus of the ultrasound probe}

One of the important requirements for PAFUSion is the matching of shape, phase, and amplitude of the identified reflection artifact with the actual PA artifact for an accurate subtraction of images. In this aspect, two important points are: 1) The amplitude of PAFUSion echoes may be directly dependent on the distance of echogenic structures from the fixed focus of the US probe, 2) Acoustic attenuation of the tissue is different for PA and US signals used for PAFUSion.

If the distance from the probe to the skin surface is small (as in any epi-mode PA/US probe), the to-be-simulated PA transients are not expected to be in the elevation focal distance of the probe, which results in a distance-dependence of the pressure amplitudes. Therefore, it is expected that the relative amplitudes of the PAFUSion identified artifacts may not be similar to the actual PA artifacts. In this situation, scaling of pressure amplitudes based on the distance from the geometrical focus of the probe may be a feasible solution.

When using US signals to mimic the PA wavefields for PAFUSion, it has undergone propagation through the tissue twice, resulting in additional attenuation compared to the real PA transients that generate the reflection artifacts (frequencies of PA and US signals are also different). Therefore, it is expected that the mimicked reflection artifacts do not perfectly match the real ones. For applications requiring larger imaging depth, this problem is expected to become prominent. A depth-dependent impulse response matching filter, based on an assumed attenuation map of the tissue can be used to solve this problem. 


\subsubsection{Photoacoustic-guided focused ultrasound (PAFUSion) using physical backpropagation of photoacoustic signals}

PAFUSion using backpropagation is based on the concept that the PA wave field can be decomposed to an outward-travelling part that is detected as "true" PA signal by the transducer, and an inward-travelling part that reflects off the echogenic structures to generate reflection artifacts. At time of PA signal generation, the two wave fields are identical but move in opposite directions. Thus, backpropagation of the wave field that was detected within the time interval $[0, T]$ reproduces after time $T$ the inward travelling wave field. The US echoes obtained after backpropagation, if reconstructed based on the PAFUSion algorithm, will be similar to the reflection artifacts in the PA image. Thus, the ideal way of doing PAFUSion will be to time-reverse the acquired PA signals and insonify the tissue with the same and to process the acquired echoes using the PAFUSion algorithm. Conventional US systems are not capable of driving the transducer elements with non-periodic pulses, which was the technical challenge using PA time reversal. However, research-based US systems from Verasonics recently reported the capability of transmitting arbitrary wavefronts (http://verasonics.com/arbitrary-waveform-generation-capability/). We envisage that PAFUSion using physical backpropagation will be feasible using this capability, which then can lead to fast and efficient reflection artifact reduction (with limited or no post-processing of data) in epi-mode and interstitial biomedical PA imaging.

\subsubsection{Multi-spectral photoacoustics for reducing reflection artifacts in biomedical photoacoustic imaging}

PAFUSion can only identify and compensate for the inward propagating part of PA signals that can be mimicked using the limited range of possible US transmission angles. A typical US probe has an angular aperture in the range of $-30^{\circ}$ to $30^{\circ}$, whereas typical PA sources (cylindrical blood vessels) radiate into an angle range of $360^{\circ}$, which leads to a limitation of the method. Reflection artifacts caused by an optical absorber and an acoustic reflector positioned side by side, will be challenging to identify using PAFUSion.

Reflection artifacts generated from any angles can be potentially identified by utilizing the optical multi-wavelength response of PA signals. This idea is based on the expected spectral difference of reflection artifact signals and real PA signals, since the source of 
reflection artifacts are generally in superficial tissue layers. Hence, closely spaced PA image features with widely different spectral behavior might be a marker for the presence of reflection artifacts. For example; skin absorption is the main cause of reflection artifacts in epi-mode biomedical PA imaging. After acquiring multi-spectral PA data, pixel-by-pixel spectral analysis can be done and if there are any pixels deep in the tissue with matching spectra as that of the skin melanin layer, then they may be the reflection artifacts caused by skin layer, as the presence of melanin is expected only in the superficial tissue layers. Using this approach, it is envisaged that reflection artifacts can be identified and reduced in epimode biomedical PA imaging. This method will be of great interest for multi-spectral PA systems without US imaging and thus PAFUSion capability (custom-made PA systems with probes that are inefficient in transmitting US). 


\section{Samenvatting}

Fotoakoestische (photoacoustic, PA) of optoakoestische beeldvorming is een hybride beeldvormingsmethode die een optisch absorptiecontrast akoestisch meet via het PA effect. Dit is een fysisch fenomeen dat geabsorbeerde optische energie converteert naar akoestische energie. PA beeldvorming is één van de snelst groeiende velden binnen de biomedische optica en is naar voren getreden als een krachtige biomedische beeldvormingsmethode in het afgelopen decennium. De techniek biedt enorme potentie voor een breed scala aan klinische en preklinische toepassingen.

Omdat PA beeldvorming gebruik maakt van detectie van ultrageluid (ultrasound, US) signalen, is integratie met conventionele puls-echo US in een multimodaal systeem een logische ontwikkeling. Een PA afbeelding geplaatst over een US afbeelding kan anatomische en structurele informatie combineren met functionele informatie, bijvoorbeeld over het lokale bloedoxygenatieniveau. Handzame (handheld) dual-mode US/PA probes maken gebruik van epi-belichting, waarbij de optische componenten geïntegreerd zijn in de US probe om het weefsel te belichten van dezelfde kant als waar de PA signalen gedetecteerd worden. Handheld US/PA beeldvorming met epi-belichting heeft echter een belangrijk nadeel; een hoge fluence (totale lichtenergie per oppervlakte eenheid) direct onder en dichtbij de US probe genereert PA signalen van oppervlakkige optische absorbers (absorberende eenheden). Deze PA signalen propageren in het weefsel en reflecteren op echogene structuren om vervolgens reflectie artefacten te produceren. Deze artefacten kunnen het beeldcontrast en de diepte waarvan een beeld gevormd kan worden sterk verminderen. Daarnaast kunnen de artefacten verschijnen in belangrijke gebieden en onterecht aangezien worden voor een optische absorber; zoals een bloedvat, een ontsteking of kankerweefsel.

Reflectie artefacten zijn een belangrijke probleem in PA beeldvorming met interstitiële belichting. Bij PA met interstitiële belichting wordt gepulseerd licht in het weefsel gebracht via een optische fiber die in een naald zit. US signalen worden extern gedetecteerd. In deze situatie zullen sterke PA signalen van de optische fiber of naaldtip zich gedragen als US zenders. Deze US signalen propageren door het weefsel en reflecteren op echogene structuren 
om vervolgens reflectie artefacten te produceren. In iedere toepassing van minimaal invasieve PA beeldvorming is dit een kritiek probleem.

Deze thesis beschrijft een nieuwe methode genaamd PAFUSion. Deze naam is afgeleid van photocoustic-guided focused ultrasound. De methode is gericht op identificatie en eliminatie van reflectie artefacten in PA biomedische beeldvorming met interstitiële belichting en epi-belichting.

Hoofdstuk 2 geeft een overzicht van de grondbeginselen van PA/US beeldvorming. Ook wordt het belang beschreven van dual-mode PA/US systemen met een handheld probe. Dit overzicht besteedt in het bijzonder aandacht aan de technische aspecten van systemen die ontwikkeld zijn door verschillende groepen en de klinische toepassingsmogelijkheden van die systemen.

PAFUSion gebruikt US transmissies om de binnenwaarts propagerende PA golf (de golf die het weefsel in propageert) van optische absorbers na te bootsen, alsmede de reflectie artefacten die hierdoor veroorzaakt worden. In hoofdstuk 3 wordt proof-of-principle van dit idee gedemonstreerd met gefocusseerde US om PA golven van puntachtige optische absorbers na te bootsen en daarmee reflectie artefacten te identificeren. We hebben numerieke simulaties uitgevoerd en fantoom experimenten (met weefselachtige fantomen) gedaan om het PAFUSion idee voor het identificeren van reflectie artefacten te valideren. De resultaten laten zien dat: 1) PAFUSion met gefocusseerde US reflectie artefacten kan ontdekken in hetzelfde vlak veroorzaakt door puntachtige absorbers in epi-modus PA beeldvorming, 2) de vorm en ruimtelijke details van de geïdentificeerde artefacten redelijk goed overeenkomen met de daadwerkelijke PA artefacten, 3) reflectie artefacten dicht bij optische absorbers kunnen komen en PAFUSion hen van elkaar kan onderscheiden. Echter is in deze proof-of-principle studie geen reductie van de artefacten gedemonstreerd. Daarnaast vereist identificatie van ruimtelijk uitgebreide structuren zoals de huid een verbetering ten opzichte van de hier gebruikte methode met gefocusseerde US.

Met het oog op deze ruimtelijk verdeelde PA bronnen die gezien worden in klinische toepassingen wordt in hoofdstuk 4 een verbeterde versie van PAFUSion voorgesteld. Hier worden PA signalen terug gepropageerd om de binnenwaarts propagerende golf te imiteren en dus ook de reflectie artefacten. De terugpropagatie is op een synthetische manier uitgevoerd gebaseerd op de puls-echo acquisities na transmissie via ieder los element van de rij transducers. In dit hoofdstuk laten we zien dat reflectie artefacten geïdentificeerd kunnen 
worden door in de tijd geïnverteerde PA signalen van een bepaald element te convolueren met US data verzameld door middel van US transmissie via datzelfde element; en vervolgens de resultaten van alle elementen te superproneren. Na deze stap worden de vormen van geïdentificeerde artefacten gematcht met de vormen van de daadwerkelijke PA artefacten door middel van een matching filter gebaseerd op de impulsresponsie. De correctie vindt plaats door de geïdentificeerde artefacten af te trekken van de oorspronkelijke PA data.

Deze voorgestelde methode (welke we synthetic aperture PAFUSion noemen) is gevalideerd met fantomen en in vivo metingen op de onderarmen van gezonde vrijwilligers. Zowel de resultaten van de fantoommetingen als de in vivo metingen laten zien dat synthetic aperture PAFUSion is in staat om reflectie artefacten te identificeren en sterk te verminderen in epi-modus PA beeldvorming. Met deze methode is de intensiteit van de reflectie artefacten gereduceerd met gemiddeld ongeveer $13 \mathrm{~dB}$ in de fantoom en in vivo experimenten. In dit hoofdstuk is voor het eerst identificatie en sterke reductie van een groot aantal reflectie artefacten vlak onder de huid en in de nabijheid van oppervlakkige bloedvaten gedemonstreerd. In een conventioneel PA beeld kunnen deze artefacten makkelijk worden aangezien voor kleine bloedvaten, ontstekingen of kankerweefsel; wat het belang van PAFUSion om de artefacten te identificeren en reduceren laat zien. De resultaten in dit hoofdstuk bevestigen dat reflectie artefacten prominent zijn in klinische epi-modus PA beeldvorming, en dat PAFUSion in staat is om de artefacten sterk te reduceren om zo de beeldinterpretatie, het contrast en de diepte waarvan een beeld gevormd kan worden te verbeteren.

Wanneer de absorptie door de huid de belangrijkste oorzaak is van reflectie artefacten (wat het geval is in ieder epi-modus systeem), is het sterke PA signaal afkomstig van de huid bijna een vlakke golf, waardoor een superpositie van een schaars aantal vlakke golven voldoende zou kunnen zijn om het grootste deel van de binnenwaarts propagerende PA golf te imiteren. Deze hypothese was een aanleiding om een alternatieve methode te implementeren, waar een virtuele terugpropagatie gebaseerd is op US vlakke golf (planewave) acquisities met transmissie onder verschillende hoeken in plaats van de synthetic aperture US transmissies beschreven in hoofdstuk 4. In hoofdstuk 5 hebben we de planewave en synthetic aperture PAFUSion geïmplementeerd in een handheld US/PA beeldvormingssysteem en hebben we met beide methodes een reductie van reflectie artefacten laten zien in fantoom experimenten en in vivo metingen aan een menselijke vinger. 
Met beide methodes was eenzelfde artefact reductie te zien (13 dB), maar plane-wave PAFUSion vereist minder puls-echo acquisities wanneer sterke PA signalen afkomstig van de huid de belangrijkste oorzaak van reflectie artefacten vormen. Hoewel de PAFUSion verwerking offline gedaan is in deze studie, is het haalbaar om real-time plane-wave en synthetic aperture PAFUSion te implementeren in ieder US/PA systeem met voldoende transmissie/acquisitie beheersing. Het systeem wat gebruikt is voor deze studie kan US en PA metingen afwisselen met een hoge frame rate $(200 \mathrm{~Hz}$, gelimiteerd door de maximaal toegestane blootstelling aan licht) en we voorzien real-time PAFUSion implementatie in dit systeem voor onze toekomstige klinische studies.

Er is veel belangstelling om PA beeldvorming met interstitiële belichting te gebruiken om minimaal invasieve procedures te begeleiden. Een groot probleem bij PA beeldvorming met interstitiële belichting van brachytherapie zaadjes wordt veroorzaakt door reflectie artefacten afkomstig van het sterke signaal van de optische fiber, de naaldtip of de nabije omgeving. PA signalen van de optische fiber, de naaldtip of de nabije omgeving propageren in het weefsel en reflecteren op zaadjes om zo reflectie artefacten te genereren. Deze artefacten kunnen zich voordoen in belangrijke gebieden, het contrast verminderen en moeilijkheden veroorzaken in het interpreteren van het beeld omdat reflecties onterecht aangezien kunnen worden voor zaadjes. In hoofdstuk 6 demonstreren we het gebruik van PAFUSion met gefocusseerde US om reflectie artefacten veroorzaakt door de optische fiber of naaldtip te identificeren en elimineren in PA beeldvorming van brachytherapie zaadjes gebruikmakend van interstitiële belichting. $\mathrm{Na}$ het identificeren van de optische fiber of naaldtip is US gefocusseerd op deze diepte en is de data gereconstrueerd met het PAFUSion algoritme zoals beschreven in hoofdstuk 3, om op deze manier reflectie artefacten te identificeren. Deze geïdentificeerde artefacten zijn gebruikt om het PA beeld te corrigeren en zo een verbeterd PA beeld te verkrijgen. De methode is in staat om de visualisatie van zaadjes te verbeteren in fantoom experimenten alsmede in ex vivo experimenten met weefsel van een varken.

Omdat PAFUSion in deze context alleen een enkele gefocusseerde US meting en een subtractie stap vereist, is real-time implementatie eenvoudig in ieder systeem. Het is voor te stellen dat de PA naald in combinatie met real-time PAFUSion in staat is om de verdeling van zaadjes accuraat weer te geven. Dit kan de radiotherapeut van feedback voorzien wat betreft de naleving van het therapieplan. Naast het afbeelden van brachytherapie zaadjes is 
het voorgestelde concept bij uitstek geschikt voor alle toepassingen van PA beeldvorming met interstitiële belichting waar reflectie artefacten kunnen ontstaan door de aanwezigheid van akoestische reflectoren. Voor zover wij weten is reductie van reflectie artefacten in de context van PA beeldvorming met interstitiële belichting voor het eerst gedemonstreerd. We voorzien dat onze methode zal helpen om PA beeldvorming met interstitiële belichting in de praktijk toe te passen. 
152 


\section{Acknowledgements}

This thesis is a result of four years of $\mathrm{PhD}$ research at the Biomedical Photonic Imaging (BMPI) group, University of Twente, The Netherlands, during which I explored the magic of light and sound for medical imaging. The PhD journey has been a truly life-changing experience for me and it would not have been fruitful without the support, inspiration, and guidance that I received from many people. Now it's time to look back and express my sincere gratitude to all those intelligent and lovely human beings.

First of all, I express my deep sense of gratitude and respect to my promotor and supervisor Prof. Wiendelt Steenbergen for providing me with the chance to work on this challenging problem and guiding me to find the most appropriate solutions. Wiendelt, I truly enjoyed the trust you had on me (even when I had no solid results during my first two years) and the freedom you offered me for conducting good research and establish collaborations. I really admire your composed, ethical, and positive approach towards research, which definitely had an impact on the development of scientific attitude in me. I am deeply indebted to you for your compassion and I thank you very much for guiding me in the right direction towards writing this book.

I gratefully acknowledge the members of my graduation committee, Prof. G. Schmitz, Prof. C.L. de Korte, Prof. C.H. Slump, Dr. P.J. Brands, and Dr. A.P. Berkhoff for their efforts and time in reviewing this thesis.

I thank Dr. Srirang Manohar for introducing me to the BMPI group and the world of photoacoustics. Srirang, I started my research career as your master student and I am always thankful to you for motivating and inspiring me to do rigorous research. I admire your skills in planning research projects, scientific writing, and delivering presentations. I must admit that I learnt a lot from you during last five years. Thanks a lot for everything.

I thank Dr. Michael Jaeger for the collaborative work that has led to two chapters of this thesis. Michael, this space may not be enough to express my gratitude towards a great mentor like you. Right from the day we started collaboration until now, you have been always a pillar of support and encouragement. I will always cherish my memories at Bern, especially the late night experiments we did just before Photonics West 2016 (shouting loud when the 
artifacts disappeared during in vivo experiments and having a beer to celebrate the success and much more). I am ever indebted to you for everything.

I thank Dr. Altaf Hussain for being a great office mate, mentor, and a very good friend. This thesis wouldn't have been in this shape without your insightful discussions and help. All the trips with you (especially Maastricht and San Francisco) were filled with lot of fun; especially I am remembering the Thai food we ate from Maastricht.

Working along with industrial partners, clinicians, and researchers involved in the FULLPHASE project was indeed a great experience for me. I thank all the FULLPHASE partners for excellent inputs during our technical meetings. Special thanks to Prof. Martin Frenz for providing me the opportunity to collaborate with his lab at University of Bern, Switzerland and for spending time in reviewing my papers and conference proceedings. I express my gratitude to Dr. Khalid Daoudi and Pim van den Berg for being great project team members and giving valuable inputs for my research. I would like to take this opportunity to thank European commission for funding my master studies and $\mathrm{PhD}$ project.

I thank Dr. Peter Brands, Theo Mulders, David Sontrop, Yannick van Bavel and all other engineers from ESAOTE Europe BV for their technical help and mentoring for using MyLabOne ultrasound system. Three months stay at ESAOTE Maastricht during my first year of $\mathrm{PhD}$ really helped me to learn well about the system and program it for my specific experiments.

I thank Dr. Ivo Vellekoop for arranging weekly group meetings and giving me chance to give presentations often. Discussions during group meetings were always of great help for me to improve the quality my work.

I thank Dr. Wenfeng Xia for being so motivating and helpful during last five years. Nonsmoking coffee breaks with fresh air and BMW rides with you were all of great fun. Special thanks to Dr. Yolanda Villanueva, Peter van Es, Sjoukje Schoustra, Vinay Parameshwarappa, Lalithai Ramachandran, Jacob Staley, Dr. Samir Biswas, Dr. Michelle Heijblom, Dr. Danielle Piras, Colin Veenstra, Gerwin Osnabrugge, Dr. Steffen Resink, Wilma Petersen, Johan van Hespen, Dr. Nienke Bosschaart, Onno Mennes, Erwin Hondebrink and all current and previous members of BMPI group for being my stunning and helpful colleagues. Yolanda, thanks a lot for being a kind office mate and helping me with all Dutch domestic procedures. Peter, my wife and me used to call you Mahatma Peter; my life at Enschede was memorable and really smooth because of your support. Johan, thanks for all the lab safety 
instructions and technical help in last five years. Colin, you were a great office mate in the last part of my PhD. Sjoukje, thanks a lot for helping me with the cover page design and "samenvatting". Vinay, you are the only master student I guided during my $\mathrm{PhD}$ period. Thanks a lot for all your hard work and perseverance which led to one chapter of this thesis. Lalithai, thanks for your inputs about the FULLPHASE multi-wavelength probe and all funny Tamil conversations (kutty boss, periya boss etc).

I thank Sylvia Winters for helping me with all administrative procedures during last five years. Sylvia, I never came out of your office without a solution to my problem. Let it be flight tickets, hotel bookings, reimbursements, visa issues etc. You were always there to help with a smiling face. Thank you for all your support.

I would like to thank my friends Vinoth, Meghna, Avinash, Praveena, Gayathri, Soumya, Saravanan, Prakash-Pavitra, and my ever-charming sister Renuka for all their encouragement and support which made my stay in Europe smooth. Vinoth, thanks a lot for being a wellwisher and a stress-reliever during last 6 years. I thank my friend and brother-in-law Srikanth and sister-in-law Archana for being so helpful and supportive. I am deeply indebted to my father-in-law and mother-in-law for being so understanding, caring and for all your prayers. Special thanks to Lamia for being so generous and helpful personally in last four years. I thank my close friends back in India, Arjun, Raji, Priyaa, and Arun. Arjun, you are an amazing person, thanks for being with me in all my ups and downs in last eighteen years. I am also remembering and thanking my friends from SRM (Biomedical 2004-2008 batch and Tambaram Malayalees), HCL Technologies, Chennai, and Model polytechnic, Calicut (Medical electronics 2001-2004 batch) for being so supportive these years.

I am remembering my late grandmother who supported my education until my bachelor studies. Ammamma, thanks a lot for teaching me the value and importance of education. I would like to use this opportunity to thank all my teachers in my academic career for activating hunger for knowledge and wisdom in me, and for helping me to become a better individual. Thanks for everything.

Words are powerless to express my gratitude towards my wife and son. Srinidhi, I finished this thesis on time only because of your sacrifices, kindness, and affection towards me. I am ever indebted to you my super-woman. Thanks a ton for never letting me down. Vidyut, my fluffy little bundle of joy, thanks a lot for being acoustically cooperative during nights, which 
helped me to finish writing this book on time. Your cute smile helped me to beat all the deadlines and pressure I had during the last period of $\mathrm{PhD}$.

I express my thanks to my brother Nithin, and sister-in-law Jijina for their support and motivation. Finally, I acknowledge my parents for showing faith in me and giving me liberty to choose what I desired. I salute you both for the selfless love, care, pain and sacrifices you did to shape up my life. Achaa, thanks for teaching me the importance of being a good human being. I value your principles and still believe that you are around and blessing me to achieve heights. Achan and amma, you both made me who I am. Words are not enough to express my gratitude for that.

\section{Mithene Kanigie A jeth Singh}

Enschede, December 2016 


\section{About the author}

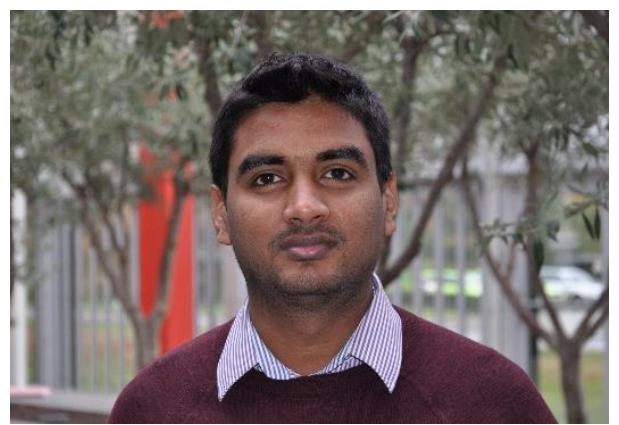

Mithun Kuniyil Ajith Singh was born on the $14^{\text {th }}$ of June 1985 in Kozhikode, Kerala, India. He went to N.S.S Higher Secondary School, Kozhikode for his elementary and high school education. He obtained a 3-year technical diploma in Medical Electronics from Model Polytechnic College, Kozhikode in 2004.

From May 2004 to January 2005, he worked as a sales and service engineer for the dealers of Karl Storz endoscopes in India. He received his Bachelor of Technology in Biomedical Instrumentation and Engineering from SRM University, Chennai in 2008. From October 2008 to July 2010, he worked as a software engineer at HCL Technologies, where he was part of the offshore team developing and testing embedded software for medical devices. In September 2010, he started his graduate studies under the prestigious Erasmus Mundus scholarship program and earned his MSc in Molecular Nano-Biophotonics from Ecole Normale Supérieure de Cachan, France in 2012. His master thesis was performed at Biomedical Photonic Imaging group (BMPI), University of Twente, The Netherlands, under the guidance of Dr. Srirang Manohar, and was focused on the design and development of interface electronics for the photoacoustic mammoscope (PAM).

From January 2013 to December 2016, he performed research towards his $\mathrm{PhD}$ thesis at BMPI group, University of Twente, The Netherlands, under the supervision of Prof. Wiendelt Steenbergen. During his research, he developed a novel method called photoacoustic-guided focused ultrasound (PAFUSion) for identifying and eliminating reflection artifacts in biomedical photoacoustic imaging. Results of his research have been published in various international journals, books, and have been presented in numerous renowned scientific conferences. 


\section{Communications}

\section{Patents}

1. W. Steenbergen, and M. Kuniyil Ajith Singh; "Artifact reduction in photoacoustic and thermoacoustic imaging," European and US patent filed (Ref: EP 15166962.9 and PCT/NL2016/050324).

\section{Peer reviewed journal articles}

1. M. Kuniyil Ajith Singh, and W. Steenbergen; "Photoacoustic-guided focused ultrasound (PAFUSion) for identifying reflection artifacts in photoacoustic imaging," Photoacoustics 3(4), 123-131 (2015).

2. M. Kuniyil Ajith Singh, M. Jaeger, M. Frenz, and W. Steenbergen., "In vivo demonstration of reflection artifact reduction in photoacoustic imaging using synthetic aperture photoacoustic-guided focused ultrasound (PAFUSion)," Biomedical optics express 7(8), 2955-2972 (2016).

3. M. Kuniyil Ajith Singh, M. Jaeger, M. Frenz, and W. Steenbergen; "Photoacoustic reflection artifact reduction using photoacoustic-guided focused ultrasound: Comparison between plane-wave and element-byelement synthetic backpropagation approach," in preparation.

4. M. Kuniyil Ajith Singh, V. Parameshwarappa, E. Hendriksen, W. Steenbergen, and S. Manohar; "Photoacoustic-guided focused ultrasound for accurate visualization of brachytherapy seeds with the photoacoustic needle," Journal of Biomedical Optics (2016), in press.

5. W. Xia, D. Piras, M. Kuniyil Ajith Singh, J.C.G. van Hespen et al., "Design and evaluation of a laboratory prototype system for $3 \mathrm{D}$ 
photoacoustic full breast tomography," Biomedical optics express 4(11), 2555-2569 (2013).

\section{Book chapters}

1. M. Kuniyil Ajith Singh, W. Steenbergen, and S. Manohar, "Handheld Probe-Based Dual Mode Ultrasound/Photoacoustics for Biomedical Imaging," in Frontiers in Biophotonics for Translational Medicine M. Olivo, and U. S. Dinish, Eds., pp. 209-247, Springer Singapore (2016).

\section{Conference proceedings}

1. M. Kuniyil Ajith Singh, and W. Steenbergen; "PhotoAcoustic-guided Focused UltraSound imaging (PAFUSion) for reducing reflection artifacts in photoacoustic imaging," in SPIE Proceedings, Opto-Acoustic Methods and Applications in Biophotonics II 95390Q (2015).

2. M. Kuniyil Ajith Singh, M. Jaeger, M. Frenz, and W. Steenbergen; "Reflection-artifact-free photoacoustic imaging using PAFUSion (photoacoustic-guided focused ultrasound)," in SPIE Proceedings, Photons Plus Ultrasound: Imaging and Sensing, 97081R (2016).

3. W. Xia, D. Piras, M. Kuniyil Ajith Singh, J.C.G. van Hespen et al., "Design considerations for ultrasound detectors in photoacoustic breast imaging," in SPIE Proceedings, Photons Plus Ultrasound: Imaging and Sensing 8581(2013).

4. P. J. van den Berg, K. Daoudi, M. Kuniyil Ajith Singh, and Wiendelt Steenbergen., "Flow imaging using an integrated photoacoustic/ultrasound probe," in SPIE Proceedings, Photons Plus Ultrasound: Imaging and Sensing 9323(2015). 


\section{Conference contributions (oral and poster)}

1. M. Kuniyil Ajith Singh, and W. Steenbergen: Photoacoustic-guided focused ultrasound (PAFUSion) for reflection-artifact-free photoacoustic imaging, poster presented at MIRA day 2015, Grolsch Veste stadium , Enschede, The Netherlands, 3 Jun 2015.

2. M. Kuniyil Ajith Singh, and W. Steenbergen: Photoacoustic-guided focused ultrasound (PAFUSion) for reflection-artifact-free photoacoustic imaging, poster presented at 7th International Graduate Summer School: Biophotonics 2015, Island of ven, Sweden, 7 Jun 2015.

3. M. Kuniyil Ajith Singh, and W. Steenbergen: PhotoAcoustic-guided Focused UltraSound imaging (PAFUSion) for reducing reflection artifacts in photoacoustic imaging, oral presentation at Opto-Acoustic Methods and Applications in Biophotonics II , European conference on biomedical optics (SPIE/OSA ECBO) 2016, Munich, Germany, 23 Jun 2015.

4. M. Kuniyil Ajith Singh, M. Jaeger, M. Frenz, and W. Steenbergen: Reflection-artifact-free photoacoustic imaging using PAFUSion (photoacoustic-guided focused ultrasound), oral presentation at SPIE Bios, Photonics West 2016, San Francisco, United States of America, 16 Feb 2016. 
